Cochrane Database of Systematic Reviews

\title{
Telephone follow-up, initiated by a hospital-based health professional, for postdischarge problems in patients discharged from hospital to home (Review)
}

Mistiaen P, Poot E

Mistiaen P, Poot E.

Telephone follow-up, initiated by a hospital-based health professional, for postdischarge problems in patients discharged from hospital to home.

Cochrane Database of Systematic Reviews 2006, Issue 4. Art. No.: CD004510.

DOI: 10.1002/14651858.CD004510.pub3. 
TABLE OF CONTENTS

HEADER 1

ABSTRACT

PLAIN LANGUAGE SUMMARY

BACKGROUND

OBJECTIVES

METHODS

Figure 1.

RESULTS

DISCUSSION

AUTHORS' CONCLUSIONS

ACKNOWLEDGEMENTS

REFERENCES

CHARACTERISTICS OF STUDIES

DATA AND ANALYSES

Analysis 1.1. Comparison 1 Effect of TFU on anxiety in cardiac surgery patients at appr. 1 month after discharge compared to usual care, Outcome 1 Effect of TFU on anxiety in cardiac surgery patients at appr. 1 month after discharge compared to usual care.

Analysis 2.1. Comparison 2 Effect of TFU on compliance, Outcome 1 Effect of TFU on compliance in cardiac surgery patients compared to usual care.

Analysis 2.2. Comparison 2 Effect of TFU on compliance, Outcome 2 Effect of TFU on compliance (making an appointment) in ED patients compared to usual care.

Analysis 2.3. Comparison 2 Effect of TFU on compliance, Outcome 3 Effect of TFU on compliance (keeping an appointment) in ED patients compared to usual care.

Analysis 3.1. Comparison 3 Effect of TFU on knowledge in cardiac patients compared to control condition, Outcome 1 Effect of TFU on knowledge in cardiac patients at around 6 weeks post discharge compared to control condition.

Analysis 4.1. Comparison 4 Effect of TFU on readmissions, Outcome 1 Effect of TFU on readmissions in cardiac patients compared to usual care.

Analysis 4.2. Comparison 4 Effect of TFU on readmissions, Outcome 2 Effect of TFU on readmissions in surgery patients compared to control condition.

Analysis 5.1. Comparison 5 Effect of TFU on ED visits in surgery patients compared to control condition, Outcome 1 Effect of

TFU on ED visits in surgery patients compared to control condition.

ADDITIONAL TABLES

APPENDICES

WHAT'S NEW

HISTORY

CONTRIBUTIONS OF AUTHORS

DECLARATIONS OF INTEREST

SOURCES OF SUPPORT

INDEX TERMS 
[Intervention Review]

\section{Telephone follow-up, initiated by a hospital-based health professional, for postdischarge problems in patients discharged from hospital to home}

Patriek Mistiaen ${ }^{1}$, Else Poot ${ }^{2}$

1NIVEL, Netherlands Institute for Healthcare Services Research, Utrecht, Netherlands. ${ }^{2}$ Netherlands Centre for Excellence in Nursing (LEVV), Utrecht, Netherlands

Contact address: Patriek Mistiaen, NIVEL, Netherlands Institute for Healthcare Services Research, PO Box 1568, Utrecht, 3500 BN, Netherlands.p.mistiaen@nivel.nl.

Editorial group: Cochrane Consumers and Communication Group.

Publication status and date: Edited (no change to conclusions), published in Issue 1, 2010.

Citation: Mistiaen P, Poot E. Telephone follow-up, initiated by a hospital-based health professional, for postdischarge problems in patients discharged from hospital to home. Cochrane Database of Systematic Reviews 2006, Issue 4. Art. No.: CD004510. DOI: 10.1002/14651858.CD004510.pub3.

Copyright @ 2010 The Cochrane Collaboration. Published by John Wiley \& Sons, Ltd.

\section{A B S T R A C T}

\section{Background}

It is known that many patients encounter a variety of problems in the first weeks after they have been discharged from hospital to home. In recent years many projects have addressed discharge planning, with the aim of reducing problems after discharge. Telephone followup (TFU) is seen as a good means of exchanging information, providing health education and advice, managing symptoms, recognising complications early, giving reassurance and providing quality aftercare service. Some research has shown that telephone follow-up is feasible, and that patients appreciate such calls. However, at present it is not clear whether TFU is also effective in reducing postdischarge problems.

\section{Objectives}

To assess the effects of follow-up telephone calls in the first month post discharge, initiated by hospital-based health professionals, to patients discharged from hospital to home.

\section{Search methods}

We searched the following databases from their start date to July 2003, without limits as to date of publication or language: the Cochrane Consumers and Communication Review Group's Specialised Register, the Cochrane Central Register of Controlled Trials (CENTRAL, The Cochrane Library), PubMed, EMBASE (OVID), BiomedCentral, CINAHL, ERIC (OVID), INVERT (Dutch nursing literature index), LILACS, Picarta (Dutch library system), PsycINFO/PsycLIT (OVID), the Combined Social and Science Citation Index Expanded (SCI-E), SOCIOFILE. We searched for ongoing research in the following databases: National Research Register (http://www.update-software.com/nrr/); Controlled Clinical Trials (http://www.controlled-trials.com/); and Clinical Trials (http://clinicaltrials.gov/). We searched the reference lists of included studies and contacted researchers active in this area.

\section{Selection criteria}

Randomised and quasi-randomised controlled trials of TFU initiated by a hospital-based health professional, for patients discharged home from an acute hospital setting. The intervention was delivered within the first month after discharge; outcomes were measured within 3 months after discharge, and either the TFU was the only intervention, or its effect could be analysed separately. 


\section{Data collection and analysis}

Two review authors independently assessed studies for inclusion and for methodological quality. The methodological quality of included studies was assessed using the criteria from the Cochrane Effective Practice and Organisation of Care Review Group. The data-extraction form was based on the template developed by the Cochrane Consumers and Communication Review Group. Data was extracted by one review author and checked by a second author. For as far it was considered that there was enough clinical homogeneity with regard to patient groups and measured outcomes, statistical pooling was planned using a random effects model and standardised mean differences for continuous scales and relative risks for dichotomous data, and tests for statistical heterogeneity were performed.

\section{Main results}

We included 33 studies involving 5110 patients. Predominantly, the studies were of low methodological quality. TFU has been applied in many patient groups. There is a large variety in the ways the TFU was performed (the health professionals who undertook the TFU, frequency, structure, duration, etc.). Many different outcomes have been measured, but only a few were measured across more than one study. Effects are not constant across studies, nor within patient groups. Due to methodological and clinical diversity, quantitative pooling could only be performed for a few outcomes. Of the eight meta-analyses in this review, five showed considerable statistical heterogeneity. Overall, there was inconclusive evidence about the effects of TFU.

\section{Authors' conclusions}

The low methodological quality of the included studies means that results must be considered with caution. No adverse effects were reported. Nevertheless, although some studies find that the intervention had favourable effects for some outcomes, overall the studies show clinically-equivalent results between TFU and control groups. In summary, we cannot conclude that TFU is an effective intervention.

\section{PLAIN LANGUAGE SUMMARY}

\section{Telephone follow-up by a hospital-based health professional after hospital discharge}

Many patients encounter a variety of problems in the first weeks after they have been discharged from hospital to home. Telephone follow-up, initiated by hospital-based health professionals, is considered to be a good means of exchanging information, providing health education and advice, managing symptoms, recognising complications early and giving reassurance to patients after discharge. Some research has shown that telephone follow-up is feasible, and that patients appreciate such calls. However, until now it was not clear whether telephone follow-up is also effective. Our systematic review identified 33 relevant studies, almost all of which were of low methodological quality (a major limitation of the review). We found that telephone follow-up has been applied in many patient groups. There is great variety in the ways the telephone follow-up has been performed. Many different outcomes have been measured. Some studies found effects in favour of the telephone follow-up intervention, but overall studies identified no statistically significant differences between the telephone follow-up and control groups. For as far as the results of studies could be pooled together, we could draw no firm conclusions about the effects of telephone follow-up. No studies identified adverse effects of the intervention. 


\section{B A C K G R O U N D}

We know from several primary studies and literature reviews (Bull 2000; Cole 2001; Hyde 2000; Mistiaen 1999a; Parker 2002; Shepperd 2004 ) that many patients encounter a variety of problems in the first weeks after they have been discharged from hospital to home. These problems can include: difficulty with activities of daily living, emotional problems, knowledge deficit (for example, insufficient knowledge to understand symptoms or advice), insufficient help, uncertainty and anxiety, and informational needs (patient perceives a need for more information than given). For instance, Bull (Bull 2000) states that 'people were given little information regarding their medications and condition, they had difficulty managing special diets, and they were often unclear about which activities they could engage in, or which ones they should avoid.... In addition elders in one study had difficulty in evaluating symptoms and deciding whether a symptom was related to their medical condition or to the adverse effects of medication... Unmet information needs one week following hospital discharge were reported by $80 \%$ of elders... Problems with recognising the signs of complications, managing medication, diet and other aspects of treatment contributed to hospital readmission' (p. 71). Although postdischarge problems are not always major medical problems, patients often perceive them as giving discomfort (LeClerc 2002). There is also empirical evidence that health professionals rate postdischarge problems in a different way to patients (Reiley 1996).

Although generally-accepted definitions of postdischarge problems and the postdischarge period are lacking, and may vary across illnesses and treatment procedures, research has shown that postdischarge problems are most intense in the period immediately after hospital discharge. Naylor's review (Naylor 2002) states that ' 4 to 6 weeks post discharge represents a critical period when many elders are at highest risk for poor discharge outcomes' and empirical research in a mixed population has shown that postdischarge problems are greater at 7 days post discharge than at 30 days post discharge (Mistiaen 1999b).

Moreover, in western developed countries, there is a tendency for shorter hospital stays and a shift to one-day-stay procedures, restricting the time available for health professionals to prepare patients adequately for their transfer to home and for the postdischarge period. This may increase postdischarge problems. Many projects have addressed discharge planning, with the aim of reducing problems after discharge. The focus of most discharge planning projects is selecting patients at risk of postdischarge problems as soon as possible after admission, preparing them in a timely and adequate fashion for discharge, and organising discharge arrangements. These discharge planning efforts do not resolve all problems, however (Parker 2002; Shepperd 2004). Patients need not only discharge preparation but also adequate aftercare. Aftercare is given in many different forms and may consist of several components, yet there is no scientific evidence that these aftercare efforts have clear beneficial effects (Bours 1998).

Since a large proportion of postdischarge problems relate to informational needs, and patients are reluctant to bother healthcare providers with their questions, it can be assumed that active telephone follow-up, initiated by hospital-based health professionals, may be of relevance to the problems patients face after discharge. Telephone follow-up (TFU) is seen as a good means of exchanging information, providing health education and advice, managing symptoms, recognising complications early, giving reassurance and providing quality aftercare service. Cox et al (Cox 2003) state that by telephone follow-up 'information can be reinforced, thereby increasing compliance, and ensuring the physical and emotional comfort of the patient'. Moreover, TFU is an intervention that is easy to organise and, in itself, does not cost a lot of money or time. The technology is available to almost all patients in western developed countries. Some research (Bowman 1994; Cave 1989; Keeling 1995; Kelly 1999) has shown that TFU is feasible, and that patients are satisfied with the calls (Johnson 2000d; Moran 1999; Schaeffer 2001). However, at present it is not clear whether TFU is also effective in reducing postdischarge problems. Studies so far show mixed results. For example, a randomised controlled trial of telephone follow-up versus usual care in ophthalmic surgery patients (Boter 2000) found no beneficial effects, except that patients valued the phone call. The authors of this study suggest that the no-effect might be due to outcome instruments that were not sensitive enough, or due to the non-problematic character of the patient group. But no-effect has also been demonstrated for more complex patient groups such as oncology patients (Beney 2002). On the other hand Beckie (Beckie 1989a) found TFU (versus no TFU) to enhance knowledge with regard to self-care measures and to reduce anxiety after discharge in coronary artery bypass graft patients, although this could not be confirmed in a later study by Roebuck (Roebuck 1999). Finally, Hartford and Wong (Hartford 2000) conclude their narrative literature review that 'plagued by inadequate sample size and weak designs, only two RCTs of nurse-initiated telephone follow-up in coronary artery bypass graft patients had positive results' (p.32).

Therefore, this review aimed to determine the effects of TFU delivered in the first month after discharge, initiated by hospitalbased health professionals, to patients discharged from hospital to home, with regard to psychosocial and physical outcomes in the first three months post discharge.

TFU is only one way of providing support after discharge; this review however focused solely on this form of care since Bours (Bours 1998) performed a systematic (non-Cochrane) review of multicomponent aftercare and Johnson (Johnson 2003) has prepared a Cochrane review of written and verbal information versus verbal information only for patients being discharged from acute hospital settings to home. Based on two trials, Johnson concludes that provision of verbal and written health information on discharge from hospital significantly increased knowledge and satisfaction scores. Bours states that the majority of the (seventeen) studies did not report clear beneficial effects in favour of the intervention (multicomponent aftercare) group.

\section{O B JECTIVES}

To determine the effects of follow-up telephone calls (TFU) in the first month post discharge, initiated by hospital-based health professionals, to patients discharged from hospital to home, with regard to psychosocial and physical outcomes in the first three months post discharge. The effects of TFU are compared to usual care or other types of hospital follow-up (for example, TFU initiated by primary-care-based health professionals).

To determine the effects of TFU initiated/delivered by various healthcare professionals (eg. nurse, MD, social worker, pharmacist, ...) in subgroup analyses where appropriate. 
To determine the effects of TFU initiated/delivered in various medical broad groups of patient populations (eg. all cardiac, all surgery patients, ...) in subgroup analyses where appropriate.

Although we expected to find that most TFU interventions focus on outcomes such as reassurance and informational needs, we included also other types of outcomes because of the great variety of postdischarge problems. We omitted to include patient satisfaction in the list of psychosocial outcomes in the protocol for this review (even though this outcome was discussed in the protocol background). We have therefore included satisfaction as a post hoc outcome in the review.

The following questions were addressed:

\section{Primary outcomes:}

- What are the effects of TFU initiated by a hospital-based health professional, on the psychosocial health (including uncertainty, anxiety, informational needs, mood, perceptions of coping, quality of life, social activity, satisfaction) of patients in the first three months post discharge, compared to usual care or other types of hospital follow-up?

- What are the effects of TFU on the physical health (including activities of daily living, self-care abilities, self efficacy, independence) of patients in the first three months post discharge compared to usual care or other types of hospital follow-u

\section{Secondary outcomes:}

- What are the effects of TFU on adherence of patients to recommended care in the first three months post discharge compared to usual care or other types of hospital follow-up?

- What are the effects of TFU on patient knowledge regarding disease or symptom management in the first three months post discharge compared to usual care or other types of hospital follow-up?

- What are the effects of TFU on adverse events (new morbidity, readmission) in the first three months post discharge compared to usual care or other types of hospital follow-up?

- What are the effects of TFU on service utilisation (healthcare services) in the first three months post discharge compared to usual care or other types of hospital follow-up?

\section{Factors influencing outcomes:}

Intervention-related factors:

- Does the structure/format of the TFU influence the outcomes?

- Does the type of healthcare provider (eg. doctor, nurse, social worker) of the TFU influence the outcomes?

- Does the timing of the TFU influence the outcomes?

- Does the frequency of the TFU influence the outcomes?

- Do discharge planning activities and/or aftercare interventions other than the TFU influence the outcomes?

Patient-related factors:

- Does the age of patients influence the effects of TFU?

- Does the length of hospital stay influence the effects of TFU?

- Does the medical diagnosis or procedure, carried out prior to discharge, influence the effects of TFU?
- Do disease severity and co-morbidities influence the effects of TFU?

- Does the person's home living arrangements (living alone, living with someone) influence the effects of TFU?

- Does the gender of patients influence the effects of TFU?

Other related factors:

- Does the country influence the effects of TFU?

- Does the type of hospital influence the effects of TFU?

Note: throughout this review the term 'patient' is used. Although we recognise that terms such as 'consumer', 'client', or 'person with ... condition' may be more accurate than 'patient' and preferred by consumers themselves, we think that 'patient' remains the term that is most well known internationally to denote a person that is or has been in contact with a health professional for a certain condition.

\section{METHODS}

\section{Criteria for considering studies for this review}

\section{Types of studies}

- Randomised controlled trials.

- Controlled trials.

In accordance with the definitions of the Cochrane EPOC group a study was considered to be a randomised controlled trial (RCT) if 'the participants were definitely assigned prospectively to one or two (or more) alternative forms of health care using a process of random allocation (eg. random number generation, coin flips)' and a study was considered to be a controlled trial if participants were 'definitely assigned prospectively to one or two (or more) alternative forms of health care using a quasi-random allocation method (eg. alternation, date of birth, patient identifier) or possibly assigned prospectively to one or two (or more) alternative forms of health care using a process of random or quasi-random allocation'.

\section{Types of participants}

- All patients discharged from an acute hospital setting (including emergency departments and one-day-stay procedures) to home (including a relative's home but excluding nursing homes or convalescence homes).

- All ages.

\section{Types of interventions}

\section{Experimental intervention}

Telephone follow-up (TFU) initiated by a hospital-based health professional (medical, nursing, social work, pharmaceutical, ...) to a patient who is discharged to his/her own home setting (including a relative's home). The TFU has to be performed at least once within the first month after discharge. The TFU may have any kind of structure: for instance completely open ('how are you doing?') or completely structured. The TFU may contain one or more elements such as gathering of information, giving reassurance, giving advice on several topics, counseling, referral where required, etc.

The TFU has, in principle, to be targeted to the patients themselves. In cases where the patients themselves are not able to talk on the phone (eg. very young children, very sick people, patients

Telephone follow-up, initiated by a hospital-based health professional, for postdischarge problems in patients discharged from hospital 
with severe Alzheimer's disease) on one or more occasions when the TFU is delivered, these studies are included. On the dataextraction sheet the extent was noted to which the TFU was indirect, and separate analyses were conducted if appropriate for studies in which the intervention for the entire research population was delivered directly to the patients, and for studies in which the TFU was (partly) delivered to relatives/caregivers. We excluded studies in which the TFU is intended primarily to address the problems of caregivers rather than of patients.

The TFU may be delivered as the only aftercare intervention, or may be part of a multi-component discharge planning or aftercare intervention, but only if the studies report data on the effects of the TFU component, or its effects can be isolated and analysed to some degree.

\section{Control intervention}

Usual care, or other types of hospital follow-up.

\section{Types of outcome measures}

In the protocol for this review we established that we would seek and report data on the outcomes listed below. It is possible that other researchers may categorise these outcomes differently. However, the complexity and the heterogeneity of this field means that we have had to choose one approach to apply to this review.

\section{Primary outcomes}

Psychosocial health of patients, including:

- uncertainty;

- anxiety (and including depression where measured with the same instrument);

- informational needs;

- mood;

- coping;

- quality of life;

- social activity;

- satisfaction (post hoc outcome, see Objectives).

Physical health of patients, including:

- level of activities of daily living (ADL)/functional status;

- self-care abilities (an outcome generally used to mean self-care activities);

- self efficacy (an outcome measured using Bandura's (Bandura 1977) concept of self-efficacy, and referring to beliefs in one's capabilities to organise and execute the courses of action required to produce given attainments)

- independence.

\section{Secondary outcomes}

Other consumer oriented outcomes, including:

- treatment adherence;

- knowledge of disease and symptom management;

- adverse effects (eg. complications, infection, readmission (ie, data reported from the patient's perspective)).

Health service delivery oriented outcomes, including:
- hospital readmission (ie. data reported from the perspective of the health service);

- health services utilisation.

The outcomes had to be measured at least once within the first three months post discharge. Since there is no generally-accepted definition of what a postdischarge period means, and the duration of postdischarge problems may vary for different illnesses and treatment procedures, the choice of a time period for study had to be arbitrary. However there is evidence, as stated earlier, that most postdischarge problems occur in the period immediately after discharge. Moreover three months is a period for which it is reasonable to assume that outcomes can be related to the intervention in the first month after discharge; it is not likely that if effects were not found in this immediate postdischarge time frame, effects would be found later.

No restrictions were made with regard to the measurement tools used, but psychometric properties were recorded.

This review is limited to outcomes in patients themselves; possible outcomes in carers or relatives are not included.

\section{Search methods for identification of studies}

In August 2003 we searched the following databases, all from their original start date until July 2003:

- Cochrane Consumers and Communication Review Group's Specialised Register,

- Cochrane Central Register of Controlled Trials (CENTRAL, The Cochrane Library),

- PubMed,

- EMBASE (OVID)

- BiomedCentral,

- CINAHL,

- ERIC (OVID)

- INVERT (Dutch nursing literature index),

- LILACS,

- Picarta (Dutch library system),

- PsyciNFO/PsycliT (OVID)

- Combined Social and Science Citation Index Expanded (SCI-E), and

- SOCIOFILE.

We had planned to search the Cochrane EPOC Review Group's Specialised Register and the System for Information on Grey Literature in Europe (SIGLE), but SIGLE was no longer available in any European library and we were unable to access the EPOC Specialised Register.

We used the highly-sensitive strategy for the retrieval of controlled trials in PubMed, as proposed by Robinson and Dickersin (Robinson 2002) and supported by the Dutch Cochrane Center (Appendix 1). For PubMed, the controlled trials strategy was combined by AND with topic-specific strategy detailed in Appendix 2

We made appropriate variations of the PubMed strategy for the other databases; the strategies are listed at Appendices 3 to 13.

We located additional references by searching the reference lists of included studies and by contacting individuals known to be active

Telephone follow-up, initiated by a hospital-based health professional, for postdischarge problems in patients discharged from hospital $\mathbf{5}$ to home (Review)

Copyright (c) 2010 The Cochrane Collaboration. Published by John Wiley \& Sons, Ltd. 
in the field of discharge and/or telephone care. In September 2004 we performed a forward search based on the included papers in the Science Citation Index, to find more recent papers that cited one or more of the already included studies.

We sought to identify ongoing research by searching the following databases in August 2003:
- National Research Register (http://www.update-software.com/ nrr/);

- Controlled Clinical Trials (http://www.controlled-trials.com/);

- Clinical Trials (http://clinicaltrials.gov/).

We did not limit the search with regard to language or publication date. The search process is presented graphically in Figure 1.

Figure 1. Figure 1: Inclusion Process

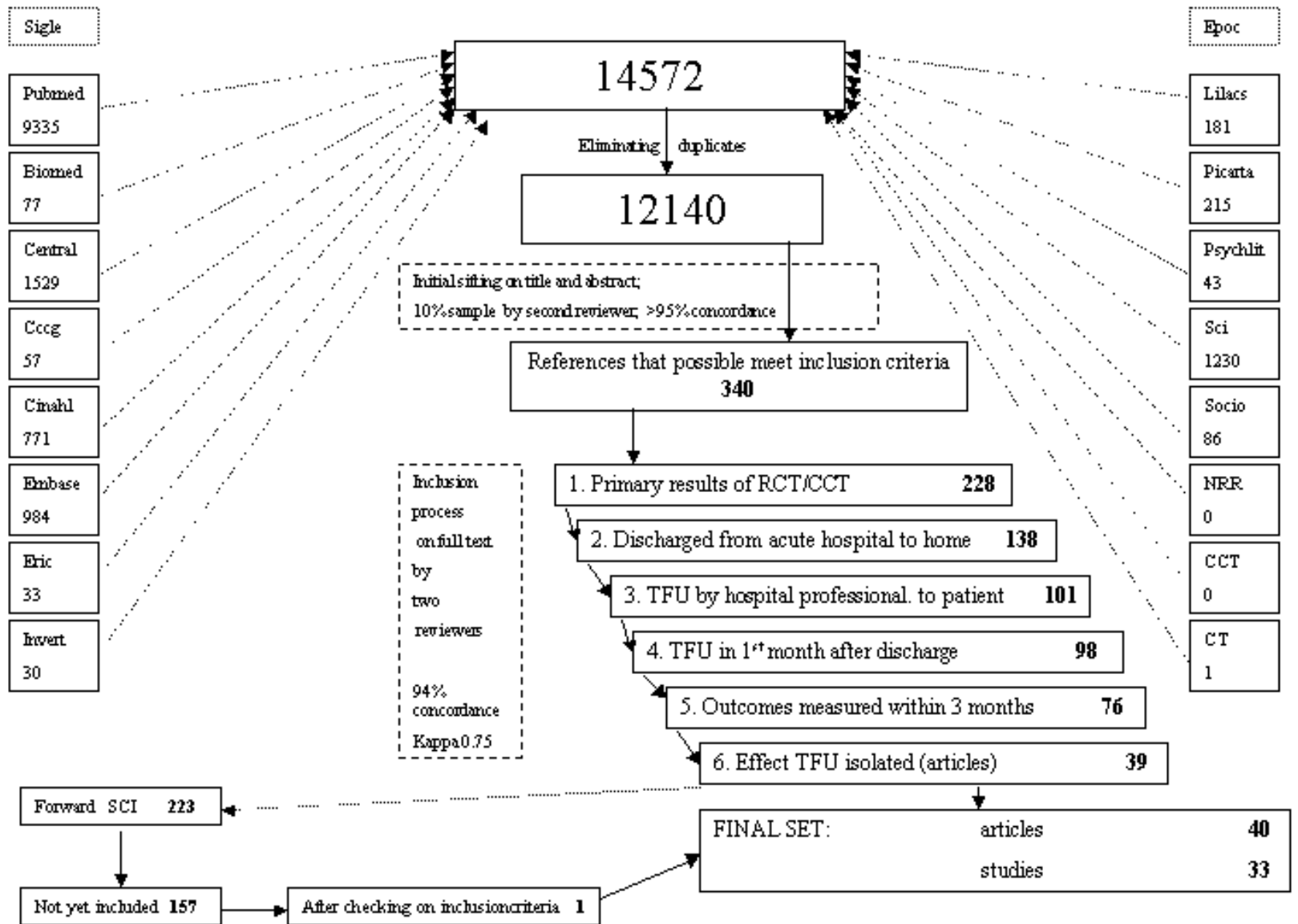

\section{Data collection and analysis}

Electronic searching in the 16 databases yielded a total of 14,572 citations and, after elimination of duplicates, 12,140 citations were left for initial sifting. This very large number of references is due to the fact that the telephone is used in many studies to collect data. It was impossible to make a distinction in the search strategies between telephone as data collection method and telephone as an intervention.

Throughout the review process, the review authors were not blind to authorship of trials.

\section{Stage 1: initial sifting}

Two authors (PM, EP) independently checked a $10 \%$ random sample of these references, and as agreement between authors was more than $95 \%$, further sifting at this stage was conducted by PM only. If the agreement whether to exclude studies between the two authors on the $10 \%$ sample was lower than $95 \%$, it was planned the second author would proceed to check the other $90 \%$ of the sample. Where there was insufficient information from the title and/or abstract to determine relevance, we ordered the article in full text and proceeded to the second stage. This initial sifting against the inclusion criteria based on the title and abstract resulted in 340 potentially-relevant references.

\section{Stage 2: inclusion procedure}

We retrieved these 340 citations in full text, and assessed them against the six inclusion criteria as follows:

- (randomised) controlled trial;

- research participants are patients discharged from hospital to their own home;

Telephone follow-up, initiated by a hospital-based health professional, for postdischarge problems in patients discharged from hospital 
- intervention must be at least one TFU call initiated by a hospitalbased health professional and, in principle, directed to the patients themselves;

- intervention has to take place at least once within the first month after hospital discharge;

- outcomes have to be measured at least once within the first three months after hospital discharge; and

- if the TFU is part of multi-component intervention, the study reports data on the effects of the TFU-component, or its effects can be isolated and analysed to some degree.

For each study the criteria were judged from top to bottom; from the moment a criterion was not met no further assessment was made relating to the subsequent criteria.

At this second stage, all studies were checked by two review authors independently. Inter-rater agreement in this process was $94 \%$ with a kappa-coefficient of 0.75 . We resolved disagreement on inclusion or exclusion was resolved by discussion. If no agreement could be reached, it was planned a third author would decide.

The process of searching and assessing studies against the review's inclusion criteria resulted in a set of 39 articles, describing 32 studies. We conducted a forward search with these 39 references in the combined Social and Science Citation Index (search date: 4 September 2004). The 39 references were cited 293 times in 223 different articles, of which 66 were already in the data-set of 2003. The remaining 157 references were checked against the inclusion criteria. Only one study (Tranmer 2004) met the criteria and was added to the final set of included studies, bringing the total to 33 included studies discussed in 40 papers. We present the inclusion process schematically at Figure 1.

The main reasons for the exclusion of studies were as follows: the study did not present results from a (randomised) controlled tria (37\%); the study did not concern patients discharged from hospital (30\%); the intervention under investigation was not TFU (12\%); or the study did not meet the other inclusion criteria. We provide additional details in the table Characteristics of excluded studies.

\section{Stage 3: data extraction}

We developed a data extraction sheet (based on the Cochrane Consumers and Communication Review Group's data extraction template), pilot-tested it on ten randomly-selected included studies, and refined it accordingly. One review author (PM) extracted the following data from included studies and the second author (EP) checked the extracted data:

- study population (diagnosis, co-morbidities, hospital procedures, age, gender-ratio, length of stay, family support, inclusion and exclusion criteria);

- study environment (type of hospital, country);

- study methods (design, randomisation procedure);

- intervention (provider, structure, content, time, frequency, duration, who answered the phone (patient or relative));

- co-interventions (discharge preparation, other forms of aftercare);

- control intervention (usual care description, TFU by others);

- outcomes (type of outcome, measurement tool (type, psychometrics), timing and frequency of assessment);
- results (mean and range at the different measurement moments post discharge, for both experimental and control group);

- conclusions (as stated by the study authors);

- limitations of study and other remarks.

Disagreements were resolved by discussion between the two review authors; if no agreement could be reached, it was planned a third author would decide.

We contacted five authors (Boter 2000; Gortner 1990; Hartford 2002; Jerant 2001; Ouellet 2003) for further information. All responded and one (Hartford 2002) provided numerical data that had only been presented graphically in the published paper.

\section{Stage 4: assessment of methodological quality}

We assessed the methodological quality of included studies using the criteria from the Cochrane Effective Practice and Organisation of Care Review Group (Alderson 2002). This list contains seven criteria to evaluate randomised controlled trials (RCTs) and controlled clinical trials (CCTs): concealment of allocation, followup of professionals, follow-up of patients, blinded assessment of primary outcomes, baseline measurement, reliable primary outcome measures, and protection against contamination. Further, as outlined in the Cochrane Handbook (Clarke 2003), we grouped studies into three categories: A (low risk of bias = all criteria met), $\mathrm{B}$ (moderate risk of bias = at least four of the criteria met) and C (high risk of bias = less than four of the criteria met).

Two review authors conducted the quality assessment independently. Disagreements were resolved by discussion between the two review authors; if not agreement could be reached, it was planned a third review author would decide. In some cases an editor of the Cochrane Consumers and Communication Review Group had input to clarifying the quality assessment for particular studies.

The EPOC quality assessment rating of each study can be found in the table Characteristics of included studies.

The quality of included studies was used to inform the discussion of the review's findings.

\section{Stage 5: analysis}

The primary analysis was a comparison of TFU with usual care or with other types of hospital follow-up, for each of the questions outlined in the review's objectives.

We grouped studies in different ways: according to similarity of intervention, according to broad groups of patient populations (eg. all cardiac patients, all surgery patients) and according to the outcomes measured. The broad groupings of patient populations were based on the similarity of issues that these patients face when they are discharged from hospital. All comparisons that were attempted or made are narratively described and presented in graphs where possible. Since we expected to find significant heterogeneity in intervention modalities, research populations, outcomes and measurement tools, we only combined the study results statistically where appropriate and with inspection of the tests for homogeneity.

The meta-analytic technique depended on the outcomes reported. For all primary and secondary outcomes (excepting adverse

Telephone follow-up, initiated by a hospital-based health professional, for postdischarge problems in patients discharged from hospital 
events, hospital readmission and health services utilisation), it was anticipated that the majority would be measured and reported as continuous data. For continuous data (which used the same instrument) the weighted mean difference (WMD) and 95\% confidence intervals $(\mathrm{Cl})$ are reported. Where the studies have used different instruments to measure the same conceptual outcome, the standardised mean difference (SMD) is reported. In studies that report dichotomous data (eg. with regard to readmission), the relative risk (RR) and $\mathrm{Cls}$ are reported. We analysed all comparisons with both a fixed-effect and a random-effects model, but only the analyses with the random-effects model are presented. We paid particular attention to the possible heterogeneity in studies and the consequences of this for interpreting the results.

If appropriate, we had planned to conduct subgroup analyses:

- for gender;

- for age group (children/adults/old/old-old);

- for living status (alone/together);

- for the different healthcare professionals delivering the TFU;

- for types of hospital (university, general,...);

- for countries;

- for TFU after one-day-stay procedures versus TFU after more than one day hospital stays;

- for TFU after short hospital stays ( $<1$ week) versus TFU after longer hospital stays;

- for TFU as the only form of discharge care versus TFU as part of multi-component discharge procedures;

- for TFU given in the first week after discharge versus TFU given later than the first week after discharge;

- for TFU in which only patients themselves were involved versus TFU in which relatives answered the telephone (due to the patient's inability);

- for TFU given as a once-only intervention versus repeated TFU; and

- for TFU given to different patient categories according to the medical diagnosis or health status (eg. severe, end of life, etc.).

We had also planned to conduct sensitivity analyses by repeating the analyses excluding studies with a ' $\mathrm{C}$ ' methodological rating, by excluding unpublished studies, and by excluding studies with extreme outlying sample sizes. However, we could not perform the intended subgroup-analyses and sensitivity analyses as too few studies were available. Consequently, we can not report any meaningful results in relation to factors that may influence the outcomes.

We were not able to report on results separately for studies where the TFU was (partly) delivered to relatives/caregivers, such as in cases where the patient has severe Alzheimer's Disease, due to lack of data.

\section{Consumer views and participation}

The protocol was submitted to three participating consumers in the Cochrane Consumers and Communication Review Group for comment, in addition to the Review Group's usual external peer review process. We sought and received additional commentary from consumers in preparing the text of the final review, through the Dutch Patients and Consumers Federation (NPCF) and the Patients' Association (UK).

\section{RES U LT S}

\section{Description of studies}

Details of each study can be found in the table Characteristics of included studies. (Blanks under a heading mean there was no information on that item in the trial report.)

TFU is an intervention that is often applied and researched in discharged patients. For this review, we selected studies in which TFU was the only intervention, or in which the effect of the TFU could be differentiated from other interventions. TFU is often combined with other discharge planning interventions, as shown in several of the included studies (Al-Asseri 2001; Barnason 1995; Beckie 1989; Faulkner 2000; Garding 1988; Gortner 1990; Hagopian 1990; Jerant 2001; Mohan 1999; Munro 1994).

The main reasons for excluding studies were: study design (not a (randomised) controlled trial), participants (not about patients discharged from an hospital to their own home or not about a telephone follow-up initiated by a hospital-based health professional), or the effects of TFU could not be calculated. For further details, see additional Figure 1 and the table Characteristics of excluded studies.

\section{Patient categories}

TFU has been applied in various patient categories, as follows:

- surgery (16) (Al-Asseri 2001; Barnason 1995; Beckie 1989; Boter 2000; Emerson 2000; Fallis 2001; Faulkner 2000; Gombeski 1993; Gortner 1990; Hartford 2002; Ouellet 2003; Roebuck 1999; Samarel 2002; Touyz 1998; Tranmer 2004; Weaver 2001);

- cardiac (12) (Al-Asseri 2001; Barnason 1995; Beckie 1989; Faulkner 2000; Garding 1988; Gortner 1990; Hartford 2002; Jerant 2001; Riegel 2002; Roebuck 1999; Tranmer 2004; Weaver 2001);

- emergency department (5) (Chande 1994; Jones 1988; Nelson 1991; Ritchie 2000; Shesser 1986);

- oncology (4) (Beney 2002; Hagopian 1990; Munro 1994; Samarel 2002);

- paediatric (3) (Chande 1994; Mohan 1999; Nelson 1991);

- neurology (2) (Phillips 1999,;Phillips 2001);

- ophthalmology (1) (Boter 2000);

- diabetes (1) (Tu 1993);

- general medicine (1) (Dudas 2001), and;

- mixed (1) (Bostrom 1996).

Some studies fall into more than one category, for example, cardiac surgery patients are included in the categories of cardiac patients and surgery patients; and studies including breast cancer patients who receive surgery and/or chemotherapy fall into both the oncology and surgery categories. The large variety in patient populations also means that there is considerable clinical heterogeneity in research samples, which made it difficult to pool results across studies. However, in some patient categories there are quite large numbers of studies, as shown above.

Further characteristics of the patient population such as age ranges, gender, race, socio-economic status and comorbidity can be found in the table Characteristics of included studies, insofar as this information was provided in the trial reports. There is

Telephone follow-up, initiated by a hospital-based health professional, for postdischarge problems in patients discharged from hospital 
also considerable variety between and within studies in these population characteristics. Studies were all conducted in highincome countries (Australia, Canada, The Netherlands, Saudi Arabia, UK, USA). It is important to note that all studies included only patients who were able to speak (and moreover, able to speak the same language as the people delivering and evaluating the TFU intervention) and handle a telephone, which makes the studies' conclusions less generalisable.

A total of 5110 patients is analysed in the 33 studies, varying in studies between 27 (Emerson 2000) and 842 (Jones 1988) with a mean of 154.8 patients per study (SD 162.4, median 118). Fifteen studies analysed less than 100 patients, twelve studies analysed between 101 and 200 patients, and six had more than 200 patients.

\section{Intervention}

There is a large variety in the way the TFU was performed in the included studies. There is variety both within and across studies in: the health professionals who undertook the TFU; the aims of the interventions; the time after discharge the calls were made; the frequency of TFU calls; the format and content of the TFU; the duration of the calls; and other aspects.

The TFU calls were made primarily by nurses (22 studies). Other professionals involved were pharmacists (Al-Asseri 2001; Beney 2002; Dudas 2001; Faulkner 2000) and physicians (Chande 1994; Touyz 1998). In three studies (Munro 1994; Ritchie 2000; Samarel 2002) different professionals were involved in performing the TFU, and in two studies (Gombeski 1993; Mohan 1999) it was not clear which health professional delivered the intervention.

The aims of the TFU can roughly be grouped in two categories: 1) to improve compliance of patients with drug regimes or appointments; or 2) to ease the transition between hospital and home and to lessen experienced distress (such as anxiety, informational needs or symptom distress) of patients in the immediate postdischarge period, by enhancing their knowledge to better manage symptoms or by giving them support and reassurance. Generally, the study authors expect that achieving the aims will lead to improved patient satisfaction, better (experienced) quality of life, fewer complications and readmissions and less resource use. Almost all studies lacked a clear theoretical framework for relating the interventions to the aims and (sequence of) outcomes and endpoints.

The frequency and timing of the intervention delivery also varied. The frequency of calls made to patients within a three-month time period after discharge varied from a single call to a series of 32 calls (Samarel 2002). Single calls were used in 14 studies, between 2 and 5 calls were applied in 7 studies (Bostrom 1996; Garding 1988; Hagopian 1990; Munro 1994; Roebuck 1999; Tu 1993; Weaver 2001), 6 to 10 calls in 7 studies (Beckie 1989; Gortner 1990; Hartford 2002; Jerant 2001; Mohan 1999; Phillips 2001; Tranmer 2004) and more than 10 calls in 5 studies (Al-Asseri 2001; Faulkner 2000; Phillips 1999; Riegel 2002; Samarel 2002). In terms of frequency of calls there are differences both between and within studies. With regard to timing, the patient was first telephoned within the first week (varying between the first and the seventh day) after discharge in 28 studies. The latest time the first call to the patient was made was four weeks after discharge. There are not only differences in timing of intervention delivery between studies, but also within studies (for example, patients were called in the first week, or between the first and third day after discharge, or between week 2 and 4 after discharge).

Some studies used a highly structured format for the TFU, with written protocols and questions that had to be asked (for example, Boter 2000; Shesser 1986; Weaver 2001), while another (Fallis 2001) does not provide any details as to how the TFU was conducted. The other included studies lay somewhere between these extremes. All articles lacked a description of the intervention sufficiently detailed and clear as to allow replication of the intervention. The control intervention, moreover, was the subject of even less description, mostly listed unhelpfully as 'usual care'.

\section{Outcomes}

Many different outcomes, falling into several categories, were measured in these studies. The number of outcomes varied between one and six per study, with a mean of 2.5 outcomes. In total, 82 outcomes were measured in the 33 included studies. (We present a more detailed list of studies categorised by outcomes in the Results section.)

Psychosocial health outcomes were measured in 20 studies. The most frequently measured outcome in this area was patient satisfaction (11), followed by anxiety (4) and depression (3). Other outcomes related to psychosocial health are: informational needs, uncertainty, mood state, coping, well-being, mental status, concerns, emotional functioning, mood disturbance, and (dimensions of) quality of life.

Physical health outcomes were measured in 10 studies. These included: activity level, functional limitations, independence, functional well-being, physical status, physical well-being, recovery, self care, self-care deficits, self efficacy, symptoms, pain, analgesic use, blood glucose level, lipid profiles, and tracking/ diagnosing pressure ulcers. Although some of these outcomes are conceptually related, the authors gave them different names. Moreover, in general, self-developed instruments were used to measure these outcomes and no two instruments were sufficiently similar to enable comparison or pooling of the results across studies.

Other consumer-oriented health outcomes were measured in 14 studies. These concerned compliance (7), knowledge (4), social functioning \& loneliness (1), symptom distress (1) and side-effects (2). (In the protocol for this review, we used the term 'adherence'. However, since all publications use the word 'compliance', we chose to use this term in the reporting of results.)

Health-services-oriented outcomes were measured in 11 studies; these considered readmissions (10), Emergency Department (ED) visits (5), unnecessary return office visits, calls to hospital and costs.

Outcomes not only varied across studies, but even when quite similar outcomes were measured in more than one study, in most cases different instruments were used.

\section{Risk of bias in included studies}

Twelve (Beckie 1989; Beney 2002; Boter 2000; Faulkner 2000; Gortner 1990; Hartford 2002; Jerant 2001; Jones 1988; Mohan 1999; Nelson 1991; Ritchie 2000; Tranmer 2004) of the 33 studies fulfilled the (strict) criteria of a randomised controlled design according to EPOC's guidelines (Alderson 2002). We describe the remaining 21 
studies as controlled clinical trials (CCTs), primarily because we could not be certain, from the papers, whether there definitely was prospectively random allocation to intervention and control groups. Self-developed instruments were used for most outcomes; psychometric quality was doubtful for most instruments. Power calculation had been done in 15 of the 33 studies. Keeping in mind the low average sample sizes there is a great chance that studies were underpowered, and effects that in reality exist were not detected.

Two review authors independently assessed the methodological quality of included studies using the EPOC criteria (Alderson 2002). The mean quality score of the first author was 2.5 and for the second author was 2.6, which was not significantly different ( $P$ $=0.72$ ). The two authors initially agreed on the methodological quality categories for 22 of the 33 included studies; the remaining 11 studies were discussed until the authors reached agreement. This resulted in 7 studies categorised as having a 'moderate risk of bias' (Beney 2002; Garding 1988; Hartford 2002; Jerant 2001; Nelson 1991; Ritchie 2000; Tranmer 2004), and the remaining 26 studies categorised as having a 'high risk of bias'. The EPOC quality criteria most often not met were: 'reliable primary outcome measures' (29/33), concealment of allocation (25/33), blinded assessment of primary outcomes' (22/33), 'baseline measurement' (15/33), protection against contamination (15/33), and follow-up of patients (9/33).

In summary, most of the studies included in this review have a high risk of bias, based on the published reports.

\section{Effects of interventions}

In 12 studies (Barnason 1995; Beney 2002; Bostrom 1996; Boter 2000; Hagopian 1990; Mohan 1999; Munro 1994; Ouellet 2003; Phillips 1999; Roebuck 1999; Tranmer 2004; Weaver 2001) the study authors conclude they found no statistically significant differences between TFU and control groups. No author reported negative effects of the TFU intervention. The authors of 21 studies conclude in favour of the TFU. It must be noted that in two studies (Emerson 2000; Gombeski 1993) the conclusions are only supported with tendencies to significance ( $P$ values that are close to 0.05). Moreover, these conclusions are sometimes only supported by significant differences later than three months after discharge, that is, outside the inclusion criteria for the review (Faulkner 2000; Jerant 2001; Phillips 2001). Of the 82 outcomes measured across the 33 studies, study authors report significant differences in favour of the TFU for 25 outcomes within the 3-month time period.

\section{Overall findings reported by quality of studies}

Categorised by methodological quality: in the 7 studies with moderate risk of bias, 18 outcomes were measured within 3 months post discharge. Of these, four studies revealed significant results (all in favour of the TFU-group) namely for knowledge (Garding 1988), for anxiety (Hartford 2002), and for compliance (Nelson 1991; Ritchie 2000). In the 26 studies with high risk of bias, 65 outcomes were measured of which 21 outcomes showed significant differences all in the favour direction for the TFU group, namely for satisfaction (Dudas 2001; Fallis 2001; Shesser 1986), for compliance (Al-Asseri 2001; Chande 1994; Jones 1988), for readmissions (Beckie 1989; Riegel 2002), for anxiety (Beckie 1989), for patient concerns (Fallis 2001), for mood disturbance (Samarel 2002), for activity level (Gortner 1990), for pain (Touyz 1998), for analgesic use (Touyz 1998), for selfcare deficits (Tu 1993), for self-efficacy (Gortner 1990), for knowledge (Beckie 1989), for calls to the hospital (Beckie 1989), for Emergency Department (ED)-visits (Dudas 2001), for drugs-related side-effects (Al-Asseri 2001) and for costs (Riegel 2002)).

Although satisfaction was not explicitly stated in our review protocol as an outcome, we did not want to exclude it because it was the most frequently measured outcome (11 studies); as noted in the Objectives, satisfaction has been added into the category of psychosocial health outcomes.

It was not possible to present results grouped by similar TFU interventions, as we had planned, because of heterogeneity in the interventions, and also lack of detailed information about the interventions. We have presented results grouped by outcomes measured, and by similar patient populations, as we had planned.

\section{Results by outcome category}

\section{Psychosocial outcomes}

Twenty studies measured some kind of pyschosocial health outcome (Al-Asseri 2001; Barnason 1995; Beckie 1989; Beney 2002; Bostrom 1996; Boter 2000; Dudas 2001; Fallis 2001; Gombeski 1993; Gortner 1990; Hagopian 1990; Hartford 2002; Jerant 2001; Munro 1994; Phillips 2001; Roebuck 1999; Samarel 2002; Shesser 1986; Tranmer 2004; Weaver 2001). Five of these studies (Beckie 1989; Dudas 2001; Fallis 2001; Hartford 2002; Shesser 1986) found favourable results in this outcome category for the TFU intervention group, namely for anxiety, satisfaction or concerns. With respect to satisfaction, however, in contrast to the three studies showing favourable effects (Dudas 2001; Fallis 2001; Shesser 1986), eight studies find no differences between intervention and control groups. Similarly, with respect to anxiety there are two studies (Beckie 1989; Hartford 2002) showing the intervention has positive effects and two studies (Hagopian 1990; Roebuck 1999) showing no difference.

\section{Physical outcomes}

Physical health outcomes were measured in ten studies (Beney 2002; Boter 2000; Gortner 1990; Hagopian 1990; Jerant 2001; Ouellet 2003; Phillips 1999; Touyz 1998; Tranmer 2004; Tu 1993). Seven of these found no differences between the intervention and control groups. However Gortner 1990 found effects in favour of the TFU group for activity level and self-efficacy; Touyz 1998 for pain and analgesic use; and Tu 1993 for self care deficits.

\section{Other consumer-related outcomes}

Other consumer-related outcomes were measured in 14 studies (Al-Asseri 2001; Barnason 1995; Beckie 1989; Beney 2002; Chande 1994; Faulkner 2000; Garding 1988; Hagopian 1990; Jones 1988; Mohan 1999; Nelson 1991; Ritchie 2000; Samarel 2002; Tu 1993). Compliance was found to be enhanced for the TFU group in five studies (Al-Asseri 2001; Chande 1994; Jones 1988; Nelson 1991; Ritchie 2000. With the exception of Al-Asseri 2001 these concern ED patients who received a single call very shortly after their ED attendance, in which they were reminded of instructions and to make an appointment with their referral doctor. Two studies (Faulkner 2000; Mohan 1999) found no differences in this compliance between groups. Knowledge was better for the TFU group in two studies (Beckie 1989; Garding 1988) and no differences in knowledge between the groups were found in two other studies (Barnason 1995; Tu 1993). Samarel 2002 assessed 
social functioning and loneliness, and found clinically equivalent results for the intervention and control group. Beney 2002 studied symptom distress and found no significant differences. Severity of side-effects of the radiotherapy, as studied by Hagopian 1990, was similar for both groups. Al-Asseri 2001 assessed the number of patients reporting drug-related side effects and found a significantly smaller number of patients reporting such side effects in the TFU group.

\section{Health services related outcomes}

Health services related outcomes were measured in 11 studies (Beckie 1989; Bostrom 1996; Dudas 2001; Emerson 2000; Fallis 2001; Ouellet 2003; Phillips 1999; Phillips 2001; Riegel 2002; Tranmer 2004; Weaver 2001). Two studies identified fewer readmissions in the intervention group (Beckie 1989; Riegel 2002) while eight studies found no differences. One study found fewer emergency department visits for the TFU group (Dudas 2001), however four studies (Fallis 2001; Ouellet 2003; Tranmer 2004; Weaver 2001) did not identify differences in this respect.

It should be noted that although we examined the studies for adverse effects of the TFU intervention, no author reported them. However, it is unclear whether study authors sought to identify adverse effects and included these outcomes in their research protocols.

\section{Results of data pooling}

We selected outcomes and patient categories for which data could be pooled quantitatively. Such pooling was only considered if similar outcomes (for example, anxiety, as a subcategory of psychosocial outcomes) were measured in at least two studies in a similar patient group (for example, patients with a cardiac condition or patients who had undergone surgery). Table 1 shows the outcomes and patient categories for which this criterion was met. (Note: cells in this table are not necessarily mutually exclusive, for instance studies of patients who had undergone cardiac surgery appear in the table under the categories of cardiac patients and of surgery patients). As outlined in the Cochrane Handbook (Clarke 2003), meta-analysis should only be considered when a group of trials is sufficiently homogeneous in terms of participants, interventions and outcomes to provide a meaningful summary. In each outcome and patient category the measurement time had to be similar, and the scales used had to be either similarly continuous or similarly dichotomous. Above all, in each category we determined whether there was sufficient clinical homogeneity to warrant data pooling. We discuss these comparisons below for each combination from Table 1.

As far as pooling was attempted, for continuous outcomes we used standardised means differences (SMDs) and a randomeffects model; for dichotomous data, we used relative risks and a random-effects model. Confidence intervals were set at $95 \%$. In all comparisons, tests were performed with regard to statistical homogeneity; this was judged (following the Cochrane Handbook (Clarke 2003)), to be acceptable, as the chi-square test value was lower than the degrees of freedom, the $P$ value of it was above 0.1 and the inconsistency test 12 was lower than $50 \%$. Finally, due to the earlier described heterogeneity in patient populations, research tools, and intervention modes, and due to the predominately low methodological quality of the studies, we stress that all reported meta-analyses have to be considered with caution.

\section{Comparisons related to psychosocial health outcomes}

A) Effect of TFU on anxiety in cardiac surgery patients at approximately one month after discharge compared to usual care Three studies measured anxiety in cardiac patients (Beckie 1989; Hartford 2002; Roebuck 1999). Two studies (Beckie 1989; Roebuck 1999) were rated as having a high risk of bias and one (Hartford 2002) as having a moderate risk of bias. All three studies involved people undergoing cardiac surgery. Anxiety was measured at a reasonably similar point in time (four and eight weeks (Hartford 2002) (data from the measurement at four weeks was used for the meta-analysis), five weeks (Roebuck 1999), and six weeks (Beckie 1989)). Three different measurement tools were used, all measuring continuous outcomes. Pooling showed a standardised mean difference of $-0.47(95 \% \mathrm{Cl}-1.28$ to 0.34$)$, which means both approaches are clinically equivalent (see Comparison 01 , Outcome $01)$. However, caution is needed since tests demonstrated large statistical heterogeneity.

B) Effect of TFU on satisfaction in cardiac (medical and surgical) patients compared to control condition

This comparison potentially involved five studies (Al-Asseri 2001; Barnason 1995; Jerant 2001; Tranmer 2004; Weaver 2001). All applied different instruments. Barnason 1995, Tranmer 2004 and Weaver 2001 measured satisfaction at approximately one month, using some kind of continuous measurement. Weaver 2001 did not present raw data and said only that there were no statistical differences. Barnason 1995 and Tranmer 2004 used different control groups, but both found no differences. Al-Asseri 2001 and Jerant 2001 measured the outcome at two months post discharge. Al-Asseri 2001 used a dichotomous outcome measure and Jerant 2001 a continuous one, which makes pooling difficult; neither found statistical differences. Heterogeneity between the studies in terms of instruments used, control groups and timing of measurement, meant that pooling could not be performed.

\section{C) Effect of TFU on satisfaction in surgery patients}

Six studies measured satisfaction in surgery patients: Al-Asseri 2001 (cardiac surgery), Barnason 1995 (cardiac surgery), Fallis 2001 (laparoscopic cholecystectomy), Gombeski 1993 (general surgery and otolaryngoly), Tranmer 2004 (cardiac surgery), and Weaver 2001 (cardiac surgery). Fallis 2001 measured satisfaction at two days post discharge, Barnason 1995, Tranmer 2004 and Weaver 2001 measured this outcome at approximately one month, Gombeski 1993 at six weeks and Al-Asseri 2001 at two months. Therefore statistical pooling was not possible due to heterogeneity in measurement times. Five studies found no differences in satisfaction and only Fallis 2001 concludes that the TFU group is statistically more satisfied. This study used a chi-square test for a continuous outcome, however, and the author states that the results should be viewed with caution because of small cell sizes. In conclusion, with regard to satisfaction in surgery patients, statistical pooling was not possible, and no single study identified favourable effects for the TFU group compared with the control groups.

\section{D) Effect of TFU on depression in cardiac surgery patients}

The next potential comparison concerns depression in cardiac surgery patients. Two studies measured this outcomes in cardiac surgery patients (Roebuck 1999; Weaver 2001). In one study the outcome was measured at one month and in the other at five weeks post discharge. These studies used two different instruments, both with a continuous scale. However, Weaver 2001 only presents a 
dichotomised result, which makes pooling impossible. Both studies found no statistically significant differences between intervention and control groups with regard to depression in cardiac surgery patients.

\section{Comparisons related to physical health outcomes}

No statistical pooling was possible in this category, as too few studies measured comparable outcomes.

\section{Comparisons related to other consumer-oriented health outcomes}

In this category, we examined compliance in several patient groups, as well as knowledge in cardiac patients.

E) Effect of TFU on compliance in cardiac surgery patients compared to usual care

Al-Asseri 2001 and Faulkner 2000 studied compliance with pilltaking in cardiac surgery patients. Al-Asseri 2001 and Faulkner 2000 were both rated as having a high risk of bias. Al-Asseri 2001 measured this outcome at 8 weeks, and Faulkner 2000 at 6 and 12 weeks after discharge. Both used dichotomous scales. For the meta-analysis, data of 6 and 8 weeks are combined; the combined effect is statistically not significant (RR $1.68,95 \% \mathrm{Cl} 0.59$ to 4.78 ) (see Comparison 02, Outcome 02). However, caution has to be taken in the interpretation of this pooling because tests demonstrated large statistical heterogeneity.

F) Effect of TFU on compliance (making and keeping an appointment) in ED patients compared to usual care

Four studies measured compliance in ED patients (Chande 1994; Jones 1988; Nelson 1991; Ritchie 2000). Nelson 1991 and Ritchie 2000 are studies with a moderate risk of bias; the others have a high risk of bias. Chande 1994 measured compliance by asking if patients had called their primary care physician and if they had filled their prescriptions. Jones 1988 measured compliance with scheduling and keeping an appointment. Nelson 1991 measued compliance by the appropriate use of follow-up care, including keeping appointments, following instructions, using the primary care centre rather than the ED for non-urgent care, and using the telephone prior to or instead of coming to the hospital for an unscheduled visit. Ritchie 2000 measured compliance in making and attending appointments. The two common points in these four studies, suitable for data pooling, are making an appointment/ calling the doctor (Chande 1994; Jones 1988; Ritchie 2000) and keeping an appointment (Jones 1988; Nelson 1991; Ritchie 2000). All four studies measured these outcomes retrospectively at different points in time, but this does not hinder comparison since they all measured making/keeping appointments that were considered to be necessary. All four studies had usual care as control condition. Jones 1988 used two additional control groups (but only the usual care control groups are taken in consideration for this comparison). The meta-analyses show effect estimates in support of the TFU intervention group, both for making an appointment (RR 1.70, 95\% Cl 0.92 to 3.14) (see Comparison 02, Outcome 02) and for keeping an appointment (RR 1.58, 95\% Cl 1.01 to 2.48) (see Comparison 02, Outcome 03). However, confidence intervals for both poolings are large and tests show considerable statistical heterogeneity.

\section{G) Effect of TFU on compliance in paediatric patients}

Compliance in paediatric patients was studied by Chande 1994, Mohan 1999 and Nelson 1991; two of these studies concern paediatric patients attending the ED and the other concerned infants requiring apnea monitoring. These samples are considered too clinically heterogeneous and were not pooled; moreover the studies of paediatric patients in the ED (Chande 1994; Nelson 1991) are already included in comparison F) above.

H) Effect of TFU on knowledge in cardiac patients compared to control condition

Three studies measured knowledge in cardiac patients (Barnason 1995; Beckie 1989; Garding 1988). Garding 1988 was rated as having a moderate risk of bias; the others as having a high risk of bias. Barnason 1995 and Beckie 1989 involve cardiac surgery patients and Garding 1988 cardiac patients who have been hospitalised for an acute myocardial infarction. All three studies used selfdeveloped instruments of which two (Beckie 1989; Garding 1988) were based on an earlier instrument of Horn and Swain. Beckie 1989 and Garding 1988 had usual care as comparison, while Barnason 1995 used two control groups (one received in-hospital teaching only, the other in-hospital teaching plus post discharge group teaching; for this meta-analysis we used data from the in-hospital teaching only control group). It is not exactly clear when the outcomes were measured, but all had to be at around four to eight weeks post discharge. The meta-analysis did not reveal a statistically favourable effect for the TFU (SMD $1.44,95 \% \mathrm{Cl}-0.25$ to 3.13) (see Comparison 03, Outcome 01), but here also tests show considerable statistical heterogeneity.

\section{Comparisons related to health services oriented outcomes}

In this section, pooling was possible for readmission data in three patient categories, and for ED visits in surgery patients.

\section{I) Effect of TFU on readmissions in cardiac patients compared to usual} care

Readmissions in cardiac patients were studied by Beckie 1989, Riegel 2002, Tranmer 2004 and Weaver 2001. Tranmer 2004 was rated as having a moderate risk of bias; the others were rated as having a high risk of bias. Riegel 2002 involved medical patients with heart failure, and the other studies involved cardiac surgery patients. Three measured readmissions by status analysis of hospital records, and one (Tranmer 2004) by patient self-report. One retrospected the first month, the second the first five weeks, the third the first six weeks and the fourth one the first three months, so possible variations between studies can be attributed to the time period, but the effects do not hamper comparisons between intervention and control. Since Weaver 2001 does not differentiate between ED visits and readmissions, this is excluded from the analysis. The pooled effect is not statistically significant (RR $0.75,95 \% \mathrm{Cl} 0.41$ to 1.36 ) (see Comparison 04, Outcome 01). Tests for statistical homogeneity are within an acceptable range.

J) Effect of TFU on readmissions in surgery patients compared to control condition

Readmissions in surgery patients were studied by Beckie 1989, Fallis 2001, Ouellet 2003, Tranmer 2004 and Weaver 2001. Only Tranmer 2004 was rated as having a moderate risk of bias; the others are at high risk of bias. Both Beckie 1989 and Tranmer 2004 were also included in comparison I, above. Four studies had usual care as comparison group; Fallis 2001 compared the TFU group to a home visit by a nurse. Three studies measured the outcome at four weeks post discharge, one at five weeks and one at six weeks. Ouellet 2003 is problematic in that the author only states

Telephone follow-up, initiated by a hospital-based health professional, for postdischarge problems in patients discharged from hospital 
that there were four readmissions in the total group and that there were no significant differences, but does not present exact data for both groups; for the meta-analysis we used two readmissions in the treatment group and two in the control group for this study. Again, as Weaver 2001 does not differentiate between ED visits and readmissions this study's data is excluded from this comparison. The pooled effect is not significant (RR $0.65,95 \% \mathrm{Cl} 0.28$ to 1.55 ) (see Comparison 04, Outcome 02) and tests for statistical homogeneity are within an acceptable range.

\section{K) Effect of TFU on readmissions in neurology patients} Readmissions in neurology patients are studied by Phillips 1999 and Phillips 2001 (in spinal cord injury patients). However readmission rates were measured over the first year and not specified for the time frame of 3 months as required for this review.

\section{L) Effect of TFU on ED visits in surgery patients compared to control condition}

The final potential meta-analysis for this review concerns surgery patients' visits to the ED ,which has been studied by Fallis 2001, Ouellet 2003, Tranmer 2004 and Weaver 2001. However Ouellet 2003 summarised ED visits together with unanticipated clinic visits and does not present specific data for ED visits, (either for the control or the intervention group) and so can not be included in this comparison. Also Weaver 2001 does not differentiate between ED visits and readmissions, and so is also excluded from the analysis. Data from Fallis 2001 and Tranmer 2004 were pooled (Fallis 2001 rated as having a high risk of bias. and Tranmer 2004 a moderate risk of bias). Tranmer 2004 had usual care as comparison group and Fallis 2001compared the TFU group to a home visit by a nurse. The pooled effect is not significant (RR $1.47,95 \% \mathrm{Cl} 0.85$ to 2.53 ) (see Comparison 05, Outcome 01). Tests for statistical homogeneity are within an acceptable range.

\section{DISCUSSION}

This review included 33 studies measuring the effects of telephone follow-up (TFU) in 5110 patients. The poor methodological quality of the included studies is a major limitation of this review. No included study had a low risk of bias, Seven had a moderate risk of bias and 26 had a high risk of bias. Moreover this review deals with a high degree of clinical diversity and statistical heterogeneity in several elements, and most studies have small sample sizes. Together, this means that drawing conclusions is very difficult, and any conclusions cannot be stated firmly.

In terms of our primary outcomes, we can draw no firm conclusions. Many different outcomes have been measured in the included studies, but only a few outcomes are measured more than one study. Moreover, many outcomes in this field are poorly defined. They are based on different and poorly described conceptual foundations. Many terms are used for the same phenomenon. There are many overlapping terms. Measurement instruments vary and are often unvalidated.

The fact that few outcomes were measured across more than one study made only limited pooling possible. As far as meta-analysis was possible, most comparisons suffered from considerable statistical heterogeneity and all pointed towards clinical equivalence.

There might be effects of the intervention which could not be shown. This may be due to poor methodological quality, (too) small sample sizes and/or insensitive instruments. We must question whether there was sufficient contrast in the studies: many studies compare TFU with usual care but do not describe what the usual care consisted of. Also, it is important to consider the extent to which patients may have received discharge preparation in hospital. It is possible that lack of contrast masks the effect of a TFU intervention.

The large heterogeneity in the interventions might also partly explain the lack of effects. Variety was evident in the people who delivered the TFU intervention, as well as in the frequency, duration, starting time, structure, and aims, and in other aspects. The extent to which the intervention is comparable across and within the studies is questionable. It seems that there is no agreement about the critical elements of an effective TFU intervention. The heterogeneity can also partly be explained by the different aims of the TFU intervention: enhancing compliance with referrals might require one form of TFU, and reducing anxiety and uncertainty, or improving a patient's knowledge of their symptoms, another form. Moreover, many studies combined several of these aims. Narrowing the inclusion criteria for this review would have improved the homogeneity of the included studies, but would have resulted not only in a very small number of included studies being identified, but also in 'laboratory' studies which do not reflect real world circumstances.

TFU can be regarded as a 'socially complex intervention', a term used by Lindsay (Lindsay 2004) to denote interventions that are characterised by actions that are difficult to define, and by varied, and difficult to control, contextual factors. Both TFU and the comparison interventions are dependent on individual professionals, individual patients, social interactions and social settings, which makes it hard to define, to standardise and to adequately describe what is being done. Many factors, therefore, may mask the effects.

Another point of discussion is given by the study of Faulkner 2000. The author found no significant differences in compliance in the short term ( 3 months) but did find differences after that period up to two years later. We should consider whether three months is too early to see the effects of TFU - but is two years realistic?

It should also be noted, however, that none of the included studies show effects in favour of the control group. Moreover, some of the studies report that patients value the TFU calls, although it seems remarkable that this is not reflected in the measured empirical outcomes. We must question, therefore, whether the scales are the right ones to measure the effects, and whether the measurement tools are sensitive enough. Also, we note that some individual studies found effects in favour of the TFU group. The Dutch Patients and Consumers Federation commented (during the preparation of this review) that patients' appreciation of the call indicates that TFU deserves a place in aftercare. To stop TFU based on the lack of firm conclusions in this review, may be to throw the baby out with the bath water. However, we strongly emphasise that questions remain about: the ideal person to deliver the intervention; the best time to start TFU; the number of follow-up calls needed and the ideal period of time for their delivery; the ideal structure and content of TFU, a possible need for variations in TFU for different patient categories; differences across countries and health systems, the nature and timing of effects to be expected of TFU, and many other issues. We need large scale, high quality studies with more comparable (and better reported) interventions and

Telephone follow-up, initiated by a hospital-based health professional, for postdischarge problems in patients discharged from hospital 13 to home (Review)

Copyright $\odot 2010$ The Cochrane Collaboration. Published by John Wiley \& Sons, Ltd. 
with sufficiently sensitive validated tools, in order to answer these questions.

\section{AUTHORS' CONCLUSIONS}

\section{Implications for practice}

Some individual studies included in this review identify some effects in favour of telephone follow-up (TFU), and no study reported adverse effects of the intervention. Nevertheless we cannot conclude that TFU is an effective intervention. Nor is there conclusive evidence to exclude TFU from discharge planning activities.

\section{Implications for research}

Research in this field should focus on the many questions as stated in the discussion. Clear and detailed descriptions of the strategies in both the intervention and control arms are needed. We note also the poor methodological quality of the included studies. For instance the criterion of reliable outcome measurement was frequently not met because outcomes were not assessed by two people, and consequently interrater agreement could not be reported; this is something that easily can be resolved. The same applies for the criteria blinded assessment of outcomes and concealment of allocation, which can quite easily be met by increased rigor in research protocols. There remain many challenges ahead, for instance to develop adequately sensitive instruments for the outcomes that can be addressed by TFU. Improved theoretical exploration of the relationship between interventions and outcomes is needed: what and when effects may be expected of TFU, and what instruments are suitable and sensitive enough to measure them? There is a need for large scale, well-designed studies with uniform and well-described interventions and outcomes.

\section{ACK N O WLEDGEMENTS}

Protocol:
We thank all referees from the Cochrane Consumers and Communication Review Group and from the NIVEL, Netherlands Institute for Healthcare Services Research, for their very useful comments on the protocol and the text of the review. We also thank the editors and staff of the Consumers and Communication Review Group; particularly Ms Judy Stoelwinder for her assistance with the search strategy for this review.

We thank NIVEL, the Netherlands Institute for Healthcare Services Research, which provided support to this review in the form of a workstation and email and internet facilities, as well as a budget of 1500 Euro for retrieving papers. The comments and support of the scientific staff of the NIVEL, and especially of Prof. Dr. J. van der Zee and Dr. A.L. Francke, were very welcome.

Review:

- Ms. Judy Stoelwinder, Consumers and Communication Review Group, assisted with the search strategies for this review.

- Ms. Anne Vicky Carlier, Netherlands Institute for Health Care Services Research, performed all interlibrary loans.

- Mr. Rob Scholten, Dutch Cochrane Center, checked the statistical analyses.

- Ms. Atie Schipaanboord commented on the text of the review from the perspective of the Dutch Patients and Consumers Federation.

- Mr. Jouke van der Zee and Ms. Anneke Francke, Director and Program Coordinator of the Netherlands Institute for Health Care Services Research, gave general scientific advice on the review.

- The scientific committee of the Netherlands Institute for Health Care Services Research commented the protocol and the text of the review.

- The editors and staff of the Consumers and Communication Review Group, and especially Dr. Megan Prictor and Dr. Sophie Hill, gave general advice, comments and above all support in writing the protocol and the text of the review. 


\section{R E F E R E N C E S}

\section{References to studies included in this review}

Al-Asseri 2001 \{published data only\}

Al Asseri AA, Al Achi A, Greenwood R. Counseling and postdischarge care. Saudi Pharmaceutical Journal 2001;9(2):119-21. [MEDLINE: 316]

\section{Barnason 1995 \{published data only\}}

Barnason S, Zimmerman L. A comparison of patient teaching outcomes among postoperative coronary artery bypass graft (CABG) patients. Progress in Cardiovascular Nursing 1995;10(4):11-20. [MEDLINE: 7566]

\section{Beckie 1989 \{published data only\}}

Beckie T. A supportive-educative telephone program: Impact on knowledge and anxiety after coronary artery bypass graft surgery. Heart \& Lung: Journal of Critical Care 1989;18(1):46-55. [MEDLINE: 964

\section{Beney 2002 \{published data only\}}

Beney J, Devine EB, Chow V, Ignoffo RJ, Mitsunaga L, Shahkarami M, et al. Effect of telephone follow-up on the physical well-being dimension of quality of life in patients with cancer. Pharmacotherapy 2002;22(10):1301-11. [MEDLINE: 1611]

\section{Bostrom 1996 \{published data only\}}

Bostrom J, Caldwell J, McGuire K, Everson D. Telephone followup after discharge from the hospital: does it make a difference? Applied Nursing Research 1996;9(2):47-52. [MEDLINE: 6948]

\section{Boter 2000 \{published and unpublished data\}}

Boter H, Mistiaen P, Groenewegen I. A randomized trial of a Telephone Reassurance Programme for patients recently discharged from an ophthalmic unit. Journal of Clinical Nursing 2000;9(2):199-206. [MEDLINE: 3631]

\section{Chande 1994 \{published data only\}}

Chande VT, Exum V. Follow-up phone calls after an emergency department visit. Pediatrics 1994;93(3):513-4. [MEDLINE: 7932]

Dudas 2001 \{published data only\}

* Dudas V, Bookwalter T, Kerr KM, Pantilat SZ. The impact of follow-up telephone calls to patients after hospitalization. American Journal of Medicine 2001;111(9 Suppl. 2):26S-30S. [MEDLINE: 231]

Dudas V, Bookwalter T, Kerr KM, Pantilat SZ. The impact of follow-up telephone calls to patients after hospitalization. Disease-a-Month 2002;48(4):239-48.

\section{Emerson 2000 \{published data only\}}

Emerson C, Gibbs L, Harper S, Woodruff C. Effect of telephone followups on post vasectomy office visits. Urology Nursing 2000;20(2):125-7, 131. [MEDLINE: 2249]

\section{Fallis 2001 \{published data only\}}

Fallis WM, Scurrah D. Outpatient laparoscopic cholecystectomy: Home visit versus telephone follow-up. Canadian Journal of Surgery 2001;44(1):39-44. [MEDLINE: 382]
Faulkner 2000 \{published data only\}

Faulkner MA, Wadibia EC, Lucas BD, Hilleman DE. Impact of pharmacy counseling on compliance and effectiveness of combination lipid-lowering therapy in patients undergoing coronary artery revascularization: a randomized, controlled trial. Pharmacotherapy 2000;20(4):410-6. [MEDLINE: 4181]

\section{Garding 1988 \{published data only\}}

Garding BS, Kerr JC, Bay K. Effectiveness of a program of information and support for myocardial infarction patients recovering at home. Heart \& Lung: Journal of Critical Care 1988;17(4):355-62. [MEDLINE: 967]

\section{Gombeski 1993 \{published data only\}}

Gombeski WR, Jr, Miller PJ, Hahn JH, Gillette CM, Belinson JL, Bravo LN, et al. Patient callback program: a quality improvement, customer service, and marketing tool. Journal of Health Care Marketing 1993;13(3):60-5. [MEDLINE: 7919]

Gortner 1990 \{published data only (unpublished sought but not used)\}

Gortner SR, Jenkins LS. Self-efficacy and activity level following cardiac surgery. Journal of Advanced Nursing 1990;15(10):1132-8. [MEDLINE: 9009]

\section{Hagopian 1990 \{published data only\}}

Hagopian GA, Rubenstein JH. Effects of telephone call interventions on patients' well-being in a radiation therapy department. Cancer Nursing 1990;13(6):339-44. [MEDLINE: 8973]

\section{Hartford 2002 \{published and unpublished data\}}

Hartford K, Wong C, Zakaria D. Randomized controlled trial of a telephone intervention by nurses to provide information and support to patients and their partners after elective coronary artery bypass graft surgery: effects of anxiety. Heart \& Lung: Journal of Critical Care 2002;31(3):199-206. [MEDLINE: 2225]

Jerant 2001 \{published data only (unpublished sought but not used)\}

Jerant AF, Azari R, Martinez C, Nesbitt TS. A randomized trial of telenursing to reduce hospitalization for heart failure: patientcentered outcomes and nursing indicators. Home Health Care Services Quarterly 2003;22(1):1-20. [MEDLINE: 1071]

* Jerant AF, Azari R, Nesbitt TS. Reducing the cost of frequent hospital admissions for congestive heart failure: a randomized trial of a home telecare intervention. Medical Care 2001;39(11):1234-45. [MEDLINE: 2788]

\section{Jones 1988 \{published data only\}}

Jones PK, Jones SL, Katz J. A randomized trial to improve compliance in urinary tract infection patients in the emergency department. Annals of Emergency Medicine 1990;19(1):16-20. [MEDLINE: 9144]

Jones PK, Jones SL, Katz J. Improving follow-up among hypertensive patients using a health belief model intervention. Archives of Internal Medicine 1987;147(9):1557-60. [MEDLINE: 9547]

Telephone follow-up, initiated by a hospital-based health professional, for postdischarge problems in patients discharged from hospital 
Jones SL, Jones PK, Katz J. A nursing intervention to increase compliance in otitis media patients. Applied Nursing Research 1989;2(2):68-73. [MEDLINE: 9285]

Jones SL, Jones PK, Katz J. Compliance for low-back pain patients in the emergency department. A randomized trial. Spine 1988;13(5):553-6. [MEDLINE: 9446]

* Jones SL, Jones PK, Katz J. Health belief model intervention to increase compliance with emergency department patients. Medical Care 1988;26(12):1172-84. [MEDLINE: 9372]

\section{Mohan 1999 \{published data only\}}

Mohan CG, Baird TM, Super DM, Chan AK, Moore JJ. Weekly telephone contact does not enhance the compliance of home apnea monitoring. Journal of Perinatology 1999;19(7):505-9. [MEDLINE: 4318]

\section{Munro 1994 \{published data only\}}

Munro AJ, Shaw T, Clarke L, Becker L, Greenwood S. A randomized study of telephone contact following completion of radiotherapy. Clinical oncology (Royal College of Radiologists (Great Britain)) 1994;6(4):242-4. [MEDLINE: 8007]

\section{Nelson 1991 \{published data only\}}

Nelson EW, Van Cleve S, Swartz MK, Kessen W, McCarthy PL. Improving the use of early follow-up care after emergency department visits. A randomized trial. American Journal of Diseases of Children 1991;145(4):440-4. [MEDLINE: 8872]

Ouellet 2003 \{published data only (unpublished sought but not used)\}

Ouellet L, Hodgins M, Pond S, Knorr S, Geldart G. Post-discharge telephone follow-up for orthopaedic surgical patients: a pilot study. Journal of Orthopaedic Nursing 2003;7(2):87-93. [MEDLINE: 15601]

\section{Phillips 1999 \{published data only\}}

Phillips VL, Temkin A, Vesmarovich S, Burns R, Idleman L. Using telehealth interventions to prevent pressure ulcers in newly injured spinal cord injury patients post-discharge. Results from a pilot study. International Journal of Technology Assessment in Health Care 1999;15(4):749-55. [MEDLINE: 4378]

\section{Phillips 2001 \{published data only\}}

Phillips VL, Vesmarovich S, Hauber R, Wiggers E, Egner A. Telehealth: reaching out to newly injured spinal cord patients. Public Health Reports 2001;116(suppl 1):94-102. [MEDLINE: 2409]

\section{Riegel 2002 \{published data only\}}

Riegel B, Carlson B, Glaser D, Kopp Z, Romero TE. Standardized telephonic case management in a Hispanic heart failure population: an effective intervention. Disease Management \& Health Outcomes 2002;10(4):241-9. [MEDLINE: 188]

* Riegel B, Carlson B, Kopp Z, LePetri B, Glaser D, Unger A. Effect of a standardized nurse case-management telephone intervention on resource use in patients with chronic heart failure. Archives of Internal Medicine 2002;162(6):705-12. [MEDLINE: 211]

\section{Ritchie 2000 \{published data only\}}

Ritchie PD, Jenkins M, Cameron PA. A telephone call reminder to improve outpatient attendance in patients referred from the emergency department: a randomised controlled trial. Australian and New Zealand Journal of Medicine 2000;30:585-92. [MEDLINE: 12367]

\section{Roebuck 1999 \{published data only\}}

Roebuck A. Telephone support in the early post-discharge period following elective cardiac surgery: does it reduce anxiety and depression levels?. Intensive and Critical Care Nursing 1999;15(3):142-6. [MEDLINE: 4470]

\section{Samarel 2002 \{published data only\}}

Samarel N, Tulman L, Fawcett J. Effects of two types of social support and education on adaptation to early-stage breast cancer. Research in Nursing and Health 2002;25(6):459-70. [MEDLINE: 1556]

\section{Shesser 1986 \{published data only\}}

Shesser R, Smith M, Adams S, Walls R, Paxton M. The effectiveness of an organized emergency department followup system. Annals of Emergency Medicine 1986;15(8):911-5. [MEDLINE: 9660]

Touyz 1998 \{published data only\}

Touyz LZG, Marchand S. The influence of postoperative telephone calls on pain perception: a study of 118 periodontal surgical procedures. Journal of Orofacial Pain 1998;12(3):219-25. [MEDLINE: 14657]

\section{Tranmer 2004 \{published data only\}}

Tranmer J, Parry M. Enhancing postoperative recovery of cardiac surgery patients: a randomized clinical trial of an advanced practice nursing intervention. Western Journal of Nursing Research 2004;26(5):515-32.

Tu 1993 \{published data only\}

Tu KS, McDaniel G, Gay JT. Diabetes self-care knowledge, behaviors, and metabolic control of older adults: the effect of a posteducational follow-up program. The Diabetes Educator 1993;19(1):25-30. [MEDLINE: 8335]

\section{Weaver 2001 \{published data only\}}

Weaver LA, Doran KA. Telephone follow-up after cardiac surgery: facilitating the transition from hospital to home. American Journal of Nursing 2001;101(5):2400, 24QQ, 24SS. [MEDLINE: 10819]

\section{References to studies excluded from this review}

Aadalen 1998 \{published data only\}

Aadalen SP. Methodological challenges to prospective study of an innovation: interregional nursing care management of cardiovascular patients. Journal of Evaluation in Clinical Practice 1998;4(3):197-223. [MEDLINE: 5479]

\section{Aaronson 1996 \{published data only\}}

Aaronson NK, Visser-Pol E, Leenhouts GH, Muller MJ, van der Schot AC, van Dam FS, et al. Telephone-based nursing

Telephone follow-up, initiated by a hospital-based health professional, for postdischarge problems in patients discharged from hospital 
intervention improves the effectiveness of the informed consent process in cancer clinical trials. Journal of Clinical Oncology 1996;14(3):984-96. [MEDLINE: 7039]

Alcaide 1990 \{published data only\}

Alcaide MJ, Altet Gomez MN, Canela SJ, Serra ML, Garrido MP, Navas AE, et al. Influence of health education on compliance with antituberculous chemoprophylaxis in children: a community trial. Revista Clinica Espanola 1990;187(2):89-93. [MEDLINE: 9079]

\section{Alfaro 1997 \{published data only\}}

Alfaro EM, Solera J, Saez L, Castillejos ML, Serna E, Cuenca D, et al. Estimation of surgical wound infection rates by a surveillance post-discharge control program. Medicina Clinica 1997;109(8):284-8. [MEDLINE: 14774]

\section{Allen 2002 \{published data only\}}

Allen SM, Shah AC, Nezu AM, Nezu CM, Ciambrone D, Hogan J, Mor $\mathrm{V}$. A problem-solving approach to stress reduction among younger women with breast carcinoma: a randomized controlled trial. Cancer 2002;94(12):3089-100. [MEDLINE: 2061]

\section{anonymous 1995a \{published data only\}}

anonymous. Follow-up phone calls provide only moderate help for noninsulin-dependent diabetics. Modern Medicine 1995;63(6):35. [MEDLINE: 15315]

\section{anonymous 1995b \{published data only\}}

anonymous. Na het ontslag: telefonische nazorg en nazorggroepen. Verpleegkundig Perspectief 1995;11(5):74. [MEDLINE: 15312]

\section{anonymous 1995c \{published data only\}}

anonymous. Telephone follow-up after myocardial infarction. Australian Nursing Journal 1995;3(1):14. [MEDLINE: 15321]

\section{anonymous 1996a \{published data only\}} anonymous. RN calling: Oncology follow-up. American Journal of Nursing 1996;96(8):50. [MEDLINE: 15303]

\section{anonymous 1996b \{published data only\}} anonymous. Telefonische nazorg door verpleegkundigen na acuut hartinfarct. Verpleegkundig Perspectief 1996;12(1):73. [MEDLINE: 15298]

\section{anonymous 1997 \{published data only\}} anonymous. Frequent phone follow-up can improve the management of heart failure. Modern Medicine 1997;65(5):55. [MEDLINE: 15287]

\section{anonymous 1998 \{published data only\}}

anonymous. Telephone care really does work. RN 1998;61(2):16. [MEDLINE: 15281]

\section{anonymous 2001a \{published data only\}}

anonymous. Follow up with patients by telephone. ED Management 2001;13(8):89-91. [MEDLINE: 2956] anonymous 2001b \{published data only\}

anonymous. Care track. Lost \& found: reaching more patients with telephone follow-up. Joint Commission Benchmark 2001;3(5):4-5. [MEDLINE: 11112]

\section{anonymous 2001c \{published data only\}}

anonymous. More educated nurses prove cost-effective for the sickest patients. Clinical Resource Management 2001;2(10):148-9, 145. [MEDLINE: 2752]

\section{Appel 2002 \{published data only\}}

Appel PR, Bleiberg J, Noiseux J. Self-regulation training for chronic pain: can it be done effectively by telemedicine? Telemedicine Journal and e-Health 2002;8(4):361-8. [MEDLINE: 1217]

\section{Arthur 2002 \{published data only\}}

Arthur HM, Smith KM, Kodis J, McKelvie R. A controlled trial of hospital versus home-based exercise in cardiac patients. Medicine and Science in Sports and Exercise 2002;34(10):1544-50. [MEDLINE: 1646]

\section{Austin 1996 \{published data only\}}

Austin JS, Maisiak RS, Macrina DM, Heck LW. Health outcome improvements in patients with systemic lupus erythematosus using two telephone counseling interventions. Arthritis Care \& Research 1996;9(5):391-9. [MEDLINE: 738]

\section{Avlund 2002 \{published data only\}}

Avlund K, Jepsen E, Vass M, Lundemark H. Effects of comprehensive follow-up home visits after hospitalization on functional ability and readmissions among old patients. A randomized controlled study. Scandinavian Journal of Occupational Therapy 2002;9(1):17-22. [MEDLINE: 11055]

\section{Bailey 1998 \{published data only\}}

Bailey ML. Care coordination in managed care. Creating a quality continuum for high risk elderly patients. Nursing Case Management 1998;3(4):172-80. [MEDLINE: 5298]

Barsevick 2002 \{published data only\} Barsevick AM, Whitmer K, Sweeney C, Nail LM. A pilot study examining energy conservation for cancer treatment-related fatigue. Cancer Nursing 2002;25(5):333-41. [MEDLINE: 1598]

\section{Bartlett 1976 \{published data only\}}

Bartlett MH, Meyer TC. Patients receive current, concise health information by telephone. Hospitals (London) 1976;50(4):79-80, 82. [MEDLINE: 10268]

\section{Bean 1995 \{published data only\}}

Bean P, Waldron K. Readmission study leads to continuum of care. Nursing Management 1995;26(9):65, 67-8. [MEDLINE: 10371]

\section{Beard 1978 \{published data only\}}

Beard JH, Malamud TJ, Rossman E. Psychiatric rehabilitation and long-term rehospitalization rates: the findings of two research studies. Schizophrenia Bulletin 1978;4(4):622-35. [MEDLINE: 10218] 
Bedeian 1996 \{published data only\}

Bedeian K, Hively JM, Gerstman BB, Dhanoa D. Decreasing the number of recheck appointments for an urgent care clinic by using telephoned follow-up care by nurses. HMO Practice 1996;10(1):44-5. [MEDLINE: 7051]

Beebe 2001 \{published data only\}

Beebe LH. Community nursing support for clients with schizophrenia. Archives of Psychiatric Nursing 2001;15(5):214-22. [MEDLINE: 2817]

\section{Behrns 2000 \{published data only\}}

Behrns KE, Kircher AP, Galanko JA, Brownstein MR, Koruda MJ. Prospective randomized trial of early initiation and hospital discharge on a liquid diet following elective intestinal surgery. Journal of Gastrointestinal Surgery 2000;4(2):217-21. [MEDLINE: 4322]

\section{Benatar 2003 \{published data only\}}

Benatar D, Bondmass M, Ghitelman J, Avitall B. Outcomes of chronic heart failure. Archives of Internal Medicine 2003;163(3):347-52. [MEDLINE: 60]

\section{Bennett 2000 \{published data only\}}

Bennett SJ, Hays LM, Embree JL, Arnould M. Heart Messages: a tailored message intervention for improving heart failure outcomes. Journal of Cardiovascular Nursing 2000;14(4):94-105. [MEDLINE: 3956]

\section{Bergstrom 2000 \{published data only\}}

Bergstrom Y, Carlson T, Jonsson A. Nursing care for ambulatory day surgery: The concept and organization of nursing care. Ambulatory Surgery 2000;8(1):3-5. [MEDLINE: 525]

\section{Berkman 1999 \{published data only\}}

Berkman P, Heinik J, Rosenthal M, Burke M. Supportive telephone outreach as an interventional strategy for elderly patients in a period of crisis. Social Work in Health Care 1999;28(4):63-76. [MEDLINE: 4776]

\section{Berry 2002 \{published data only\}}

Berry C. Telephone follow-up by nurses reduces hospital readmissions among people with chronic heart failure. Evidence-based Healthcare 2002;6(4):152-3. [MEDLINE: 15243]

\section{Biermann 2000 \{published data only\}}

Biermann E, Dietrich W, Standl E. Telecare of diabetic patients with intensified insulin therapy. A randomized clinical trial. Studies in Health Technology and Informatics 2000;77:327-32. [MEDLINE: 3507]

\section{Biermann 2002 \{published data only\}}

Biermann E, Dietrich W, Rihl J, Standl E. Are there time and cost savings by using telemanagement for patients on intensified insulin therapy?: a randomised, controlled trial. Computer Methods \& Programs in Biomedicine 2002;69(2):137-46. [MEDLINE: 159]

\section{Blake 1990 \{published data only\}}

Blake RL, Jr, Vandiver TA, Braun S, Bertuso DD, Straub V. A randomized controlled evaluation of a psychosocial intervention in adults with chronic lung disease. Family Medicine 1990;22(5):365-70. [MEDLINE: 9032]

Blue 2001 \{published data only\}

Blue L, Lang E, McMurray JJ, Davie AP, McDonagh TA, Murdoch DR, et al. Randomised controlled trial of specialist nurse intervention in heart failure. BMJ 2001;323(7315):715-8. [MEDLINE: 2828]

\section{Booker 2000 \{published data only\}}

Booker J, Cowan RA, Logue JP, Wylie JP, Eardley A. Evaluation of a nurse-led telephone clinic in the follow-up of patients with prostate cancer. Clinical oncology (Royal College of Radiologists (Great Britain)) 2000;12(4):273. [MEDLINE: 3805]

Bostelman 1994 \{published data only\}

Bostelman S, Callan M, Rolincik LC, Gantt M, Herink M, King J, et al. A community project to encourage compliance with mental health treatment aftercare. Public Health Reports 1994;109(2):153-7. [MEDLINE: 7929]

\section{Boter 1998 \{published data only\}}

Boter H, Groenewegen I, van Dijk L, Mistiaen P. Verpleegkundigen vullen lacune in de zorg op: telefonische nazorg voor oogheelkunde patiënten. TVZ 1998;108(9):288-91. [MEDLINE: 15224]

\section{Bourbeau 2003 \{published data only\}}

Bourbeau J, Julien M, Maltais F, Rouleau M, Beaupre A Begin $R$, et al. Reduction of hospital utilization in patients with chronic obstructive pulmonary disease: a disease-specific self-management intervention. Archives of Internal Medicine 2003;163(5):585-91. [MEDLINE: 1223]

\section{Branch 1999 \{published data only\}}

Branch VK, Lipsky K, Nieman T, Lipsky PE. Positive impact of an intervention by arthritis patient educators on knowledge and satisfaction of patients in a rheumatology practice. Arthritis Care \& Research 1999;12(6):370-5. [MEDLINE: 3673]

Brandis 1998 \{published data only\}

Brandis S, Murtagh S, Solia R. The Allied Health BONE (Best Orthopaedic New Enterprise) team: an interdisciplinary approach to orthopaedic early discharge and admission prevention. Australian Health Review 1998;21(3):211-222. [MEDLINE: 6103]

Brandt 1994 \{published data only\}

Brandt K, Caldwell J. Patients and their learning needs: a new look... Telephone Nursing Care Link Project. Stanford Nurse 1994;16(1):12-3. [MEDLINE: 10423]

\section{Brooks 2002 \{published data only\}}

Brooks D, Krip B, Mangovski-Alzamora S, Goldstein RS. The effect of postrehabilitation programmes among individuals with chronic obstructive pulmonary disease. European Respiratory Journal 2002;20(1):20-9. [MEDLINE: 147]

Caison 1997 \{published data only\}

Caison WB. Alcohol and drug treatment telephone follow-up using twelve step group member volunteers: Effects on a.a. and

Telephone follow-up, initiated by a hospital-based health professional, for postdischarge problems in patients discharged from hospital 
n.a. affiliation self-efficacy and behaviors among callers and call recipients. Dissertation Abstracts International: Section B: The Sciences and Engineering 1997;58(6-B):3366. [MEDLINE: 13724]

\section{Cave 1989 \{published data only\}}

Cave LA. Follow-up phone calls after discharge. American Journal of Nursing 1989;89(7):942-3. [MEDLINE: 15208]

\section{Celestino 1998 \{published data only\}}

Celestino H, McLaughlin T, King J. Telephone evaluation of effectiveness of discharge education on self-care management following total hip replacement. Orthoscope 1998;4(1):5-7. [MEDLINE: 10598]

\section{Chong 2003 \{published data only\}}

Chong J, Herman-Stahl M. Substance abuse treatment outcomes among American Indians in the Telephone Aftercare Project. Journal of Psychoactive Drugs 2003;35(1):71-7. [MEDLINE: 14009]

\section{Chow 2001 \{published data only\}}

Chow E, Wong R, Connolly R, Hruby G, Franssen E, Fung KW, et al. Prospective assessment of symptom palliation for patients attending a rapid response radiotherapy program: feasibility of telephone follow-up. Journal of Pain \& Symptom Management 2001;22(2):649-56. [MEDLINE: 308]

\section{Cleuren 2000 \{published data only\}}

Cleuren G, Jaarsma T, Lucas C. Telefonische bereikbaarheid van een gespecialiseerd hartfalenverpleegkundige. Cordiaal 2000;21(5):143-6. [MEDLINE: 15215]

\section{Cooper 2000 \{published data only\}}

Cooper HM, Meyer DR. Outpatient ophthalmic plastic surgery: Outcomes and patient satisfaction using initial postoperative telephone call follow-up. Ophthalmic Plastic \& Reconstructive Surgery 2000;16(3):231-6. [MEDLINE: 398]

\section{Craddock 1999 \{published data only\}}

Craddock RB, Adams PF, Usui WM, Mitchell L. An intervention to increase use and effectiveness of self-care measures for breast cancer chemotherapy patients. Cancer Nursing 1999;22(4):312-9. [MEDLINE: 4722]

\section{Dale 1997 \{published data only\}}

Dale J, Crouch R. Telephone advice. It's good to talk. Health Service Journal 1997;107(5536):24-6. [MEDLINE: 6628]

\section{Dantas 2002 \{published data only\}}

Dantas RA, Aguillar OM, dos Santos Barbeira CB. Implementation of a nurse-monitored protocol in a Brazilian hospital: a pilot study with cardiac surgery patients. Patient Education \& Counseling 2002;46(4):261-6. [MEDLINE: 2328]

\section{Dardik 1997 \{published data only\}}

Dardik A, Williams GM, Minken SL, Perler BA. Impact of a critical pathway on the results of carotid endarterectomy in a tertiary care university hospital: effect of methods on outcome. Journal of Vascular Surgery 1997;26(2):186-92. [MEDLINE: 13575]
DeBusk 1985 \{published data only\}

DeBusk RF, Haskell WL, Miller NH, Berra K, Taylor CB, Berger WE, III, Lew $\mathrm{H}$. Medically directed at-home rehabilitation soon after clinically uncomplicated acute myocardial infarction: a new model for patient care. American Journal of Cardiology 1985;55(4):251-7. [MEDLINE: 9833]

DeBusk 1994 \{published data only\}

DeBusk RF, Miller NH, Superko HR, Dennis CA, Thomas RJ, Lew HT, et al. A case-management system for coronary risk factor modification after acute myocardial infarction. Annals of Internal Medicine 1994;120(9):721-9. [MEDLINE: 7864]

Dellasega 2000 \{published data only\}

Dellasega CA, Zerbe TM. A multimethod study of advanced practice nurse postdischarge care. Clinical Excellence for Nurse Practitioners 2000;4(5):286-93. [MEDLINE: 10887]

\section{Delores 2000 \{published data only\}}

Delores MJ, Gries DM, Phyall G, Barfield WD. Evaluation of an early discharge program for infants after childbirth in a military population. Military Medicine 2000;165(8):616-21. [MEDLINE: 3882]

\section{Doolittle 1997 \{published data only\}}

Doolittle GC, Harmon A, Williams A, Allen A, Boysen CD, Wittman C, et al. A cost analysis of a tele-oncology practice. Journal of Telemedicine and Telecare 1997;3 Suppl 1:20-2. [MEDLINE: 6569]

Dunn 1995 \{published data only\}

Dunn RB, Guy PM, Hardman CS, Lewis PA, Vetter NJ. Can a house call by a public health nurse improve the quality of the discharge process for geriatric patients?. Clinical Performance and Quality Health Care 1995;3(3):151-5. [MEDLINE: 7382]

Eaton 2002 \{published data only\}

Eaton J, Rasgon B, Derbin LW, Hilsinger RL, Jr, Buenavista T. Telephone appointment visits for head and neck surgery followup care. Laryngoscope 2002;112(6):1060-4. [MEDLINE: 2003]

\section{Edwards 1997 \{published data only\}}

Edwards NC, Sims JN. A randomized controlled trial of alternative approaches to community follow-up for postpartum women. Canadian Journal of Public Health Revue Canadienne de Sante Publique 1997;88:123-8. [MEDLINE: 12730]

\section{Elliott 1998 \{published data only\}}

Elliott S, Reimer C. Postdischarge telephone follow-up program for breastfeeding preterm infants discharged from a special care nursery. Neonatal Network 1998;17(6):41-5. [MEDLINE: 5335]

\section{Engelman 1994 \{published data only\}}

Engelman RM, Rousou JA, Flack III JE, Deaton DW, Humphrey CB, Ellison LH, et al. Fast-track recovery of the coronary bypass patient. Annals of Thoracic Surgery 1994;58(6):1742-6. [MEDLINE: 830] 
Estey 1990 \{published data only\}

Estey AL, Tan MH, Mann K. Follow-up intervention: its effect on compliance behavior to a diabetes regimen. The Diabetes Educator 1990;16(4):291-5. [MEDLINE: 9058]

\section{Evans 1985 \{published data only\}}

Evans RL, Halar EM, Smith KM. Cognitive therapy to achieve personal goals: results of telephone group counseling with disabled adults. Archives of Physical Medicine and Rehabilitation 1985;66(10):693-6. [MEDLINE: 9781]

\section{Ezenkwele 2003 \{published data only\}}

Ezenkwele UA, Sites FD, Shofer FS, Pritchett EN, Hollander JE. A randomized study of electronic mail versus telephone followup after emergency department visit. Journal of Emergency Medicine 2003;24(2):125-30. [MEDLINE: 1250]

\section{Faithfull 2001 \{published data only\}}

Faithfull S, Corner J, Meyer L, Huddart R, Dearnaley D. Evaluation of nurse-led follow up for patients undergoing pelvic radiotherapy. British Journal of Cancer 2001;85(12):1853-64. [MEDLINE: 155]

\section{Farrero 2001 \{published data only\}}

Farrero E, Escarrabill J, Prats E, Maderal M, Manresa F. Impact of a hospital-based home-care program on the management of COPD patients receiving long-term oxygen therapy. Chest 2001;119 (2):364-9. [MEDLINE: 13576]

\section{Ferrigno 2001 \{published data only\}}

Ferrigno RF, Bradley K, Werdmann MJ. A simple strategy for improving patient contact after ED discharge. American Journal of Emergency Medicine 2001;19(1):46-8. [MEDLINE: 402]

\section{Fitzgerald 1985 \{published data only\}}

Fitzgerald JL, Mulford HA. An experimental test of telephone aftercare contacts with alcoholics. Journal of Studies on Alcohol 1985;46(5):418-24. [MEDLINE: 979]

\section{Fleming 2002 \{published data only\}}

Fleming MF, Mundt MP, French MT, Manwell LB, Stauffacher EA, Barry KL. Brief physician advice for problem drinkers: longterm efficacy and benefit-cost analysis. Alcoholism, Clinical and Experimental Research 2002;26(1):36-43. [MEDLINE: 2522]

\section{Fowler 1992 \{published data only\}}

Fowler L. Family psychoeducation: chronic psychiatrically ill Caribbean patients. Journal of Psychosocial Nursing and Mental Health Services 1992;30(3):27-32. [MEDLINE: 8603]

\section{Frank 1986 \{published data only\}}

Frank DA, Wirtz SJ, Sorenson JR, HR. Duration of breast-feeding among low-income women: a randomized trial of the effects of commercial hospital discharge packs and hospital-based telephone counseling. American Journal of Diseases of Children 1986;140:311. [MEDLINE: 13314]

\section{Frank 1987 \{published data only\}}

Frank DA, Wirtz SJ, Sorenson JR, Heeren T. Commercial discharge packs and breast-feeding counseling: effects on infant-feeding practices in a randomized trial. Pediatrics 1987;80(6):845-54. [MEDLINE: 9517]

Frasure-Smith 1985 \{published data only\}

Frasure-Smith N, Prince R. The ischemic heart disease life stress monitoring program: impact on mortality. Psychosomatic Medicine 1985;47(5):431-45. [MEDLINE: 9783]

\section{Frasure-Smith 1991 \{published data only\}}

Frasure-Smith N. In-hospital symptoms of psychological stress as predictors of long-term outcome after acute myocardial infarction in men. American Journal of Cardiology 1991;67(2):121-7. [MEDLINE: 8914]

\section{Frasure-Smith 1992 \{published data only\}}

Frasure-Smith N, Lesperance F, Juneau M. Differential long-term impact of in-hospital symptoms of psychological stress after non-Q-wave and Q-wave acute myocardial infarction. American Journal of Cardiology 1992;69(14):1128-34. [MEDLINE: 927]

\section{Frasure-Smith 1997 \{published data only\}}

Frasure-Smith N, Lesperance F, Prince R, Verrier P, Garber RA, Juneau M, et al. Randomised trial of home-based psychosocial nursing intervention for patients recovering from myocardial infarction. Lancet 1997;350(9076):473-9. [MEDLINE: 15342]

\section{Frasure-Smith 2002 \{published data only\}}

Frasure-Smith N, Lesperance F, Gravel G, Masson A, Juneau M, Bourassa MG. Long-term survival differences among lowanxious, high-anxious and repressive copers enrolled in the Montreal heart attack readjustment trial. Psychosomatic Medicine 2002;64(4):571-9. [MEDLINE: 2029]

\section{Fretwell 1990 \{published data only\}}

Fretwell MD, Raymond PM, McGarvey ST, Owens N, Traines M, Silliman RA, Mor V. The Senior Care Study. A controlled trial of a consultative/unit-based geriatric assessment program in acute care. Journal of the American Geriatrics Society 1990;38(10):1073-81. [MEDLINE: 9012]

\section{Friedman 1998a \{published data only\}}

Friedman RH, Stollerman J, Rozenblyum L, Belfer D, Selim A, Mahoney D, Steinbach S. A telecommunications system to manage patients with chronic disease. Medinfo 1998;9 Pt 2:1330-4. [MEDLINE: 4866]

\section{Friedman 1998b \{published data only\}}

Friedman RH. Automated telephone conversations to assess health behavior and deliver behavioral interventions. Journal of Medical Systems 1998;22(2):95-102. [MEDLINE: 5762]

\section{Fukuda 1999 \{published data only\}}

Fukuda H, Muto T, Kawamori R. Evaluation of a diabetes patient education program consisting of a three-day hospitalization and a six-month follow-up by telephone counseling for mild type 2 diabetes and IGT. Environmental Health \& Preventive Medicine 1999;4(3):122-9. [MEDLINE: 531]

\section{Gagnon 1997 \{published data only\}}

Gagnon AJ, Edgar L, Kramer MS, Papageorgiou A, Waghorn K, Klein MC. A randomized trial of a program of early postpartum

Telephone follow-up, initiated by a hospital-based health professional, for postdischarge problems in patients discharged from hospital 
discharge with nurse visitation. American Journal of Obstetrics and Gynecology 1997;176(1 Pt 1):205-11. [MEDLINE: 6599]

\section{Gagnon 1999 \{published data only\}}

Gagnon AJ, Schein C, McVey L, Bergman H. Randomized controlled trial of nurse case management of frail older people. Journal of the American Geriatrics Society 1999;47(9):1118-24. [MEDLINE: 546]

\section{Gagnon 2002 \{published data only\}}

Gagnon AJ, Dougherty G, Jimenez V, Leduc N. Randomized trial of postpartum care after hospital discharge. Pediatrics 2002;109(6):1074-80. [MEDLINE: 2180]

\section{Gallagher 2003 \{published data only\}}

Gallagher R, McKinley S, Dracup K. Effects of a telephone counseling intervention on psychosocial adjustment in women following a cardiac event. Heart \& Lung: Journal of Critical Care 2003;32(2):79-87. [MEDLINE: 7]

\section{Galt 2000 \{published data only\}}

Galt K. Identifying noncompliance by combining refill audits with telephone follow-up (letter). American Journal of HealthSystem Pharmacy 2000;57(3):219. [MEDLINE: 15259]

\section{Gamboa 2002a \{published data only\}}

Gamboa A, Gomez CE, de Villar CE, Vega SJ, Lopez AR, Polo J. The special attention to re-admitted patients can be effective. Cost-benefit analysis of a new health care model. Revista Clinica Espanola 2002;202(6):320-5. [MEDLINE: 143]

\section{Gamboa 2002b \{published data only\}}

Gamboa A, Gomez CE, de Villar CE, Vega SJ, Mayoral ML, Lopez AR. A new model for medical care to multi-admitted patients. Revista Clinica Espanola 2002;202(4):187-96. [MEDLINE: 172]

\section{Garland 1992 \{published data only\}}

Garland M. Discharge follow-up by telephone. Rehabilitation Nursing 1992;17(6):339-41. [MEDLINE: 10439]

\section{Garnett 1981 \{published data only\}}

Garnett WR, Davis LJ, McKenney JM, Steiner KC. Effect of telephone follow-up on medication compliance. American Journal of Hospital Pharmacy 1981;38(5):676-9. [MEDLINE: 10091]

\section{Genev 1990 \{published data only\}}

Genev NM, McGill M, Hoskins PL, Constantino MI, Plehwe W, Yue DK, et al. Continuing diabetes education by telephone. Diabetic Medicine 1990;7(10):920-1. [MEDLINE: 8983]

\section{Gilliss 1993 \{published data only\}}

Gilliss CL, Gortner SR, Hauck WW, Shinn JA, Sparacino PA, Tompkins C. A randomized clinical trial of nursing care for recovery from cardiac surgery. Heart \& Lung: Journal of Critical Care 1993;22(2):125-33. [MEDLINE: 905] management. Health Education Research 1995;10(4):467-78. [MEDLINE: 786]

Glasgow 1996 \{published data only\}

Glasgow RE, Toobert DJ, Hampson SE. Effects of a brief office-based intervention to facilitate diabetes dietary selfmanagement. Diabetes Care 1996;19(8):835-42. [MEDLINE: 6833]

\section{Glasgow 1997 \{published data only\}}

Glasgow RE, La Chance PA, Toobert DJ, Brown J, Hampson SE, Riddle MC. Long-term effects and costs of brief behavioural dietary intervention for patients with diabetes delivered from the medical office. Patient Education \& Counseling 1997;32(3):175-84. [MEDLINE: 6015]

Glasgow 2000 \{published data only\}

Glasgow RE, Toobert DJ. Brief, computer-assisted diabetes dietary self-management counseling: effects on behavior, physiologic outcomes, and quality of life. Medical Care 2000;38(11):1062-73. [MEDLINE: 3682]

\section{Glasgow 2001 \{published data only\}}

Glasgow RE, McKay HG, Piette JD, Reynolds KD. The RE-AIM framework for evaluating interventions: what can it tell us about approaches to chronic illness management?. Patient Education \& Counseling 2001;44(2):119-27. [MEDLINE: 2986]

\section{Glasgow 2002 \{published data only\}}

Glasgow RE, Toobert DJ, Hampson SE, Strycker LA. Implementation, generalization and long-term results of the "choosing well" diabetes self-management intervention. Patient Education \& Counseling 2002;48(2):115-22. [MEDLINE: 1589]

\section{Goes 2002 \{published data only\}}

Goes I. Nazorg per telefoon na operatie wegens cervixcarcinoom. BOG info 2002, (24):12-3. [MEDLINE: 15211]

\section{Gortner 1988 \{published data only\}}

Gortner SR, Gilliss CL, Shinn JA, Sparacino PA, Rankin S, Leavitt $M$, et al. Improving recovery following cardiac surgery: a randomized clinical trial. Journal of Advanced Nursing 1988;13(5):649-61. [MEDLINE: 10486]

\section{Grancelli 2003 \{published data only\}}

Grancelli H, Varini S, Ferrante D, Schwartzman R, Zambrano C, Soifer S, et al. Randomized trial of telephone intervention in chronic heart failure (DIAL): study design and preliminary observations. Journal of Cardiac Failure 2003;9(3):172-9. [MEDLINE: 1017]

\section{Greineder 1995 \{published data only\}}

Greineder DK, Loane KC, Parks P. Reduction in resource utilization by an asthma outreach program. Archives of Pediatrics and Adolescent Medicine 1995;149(4):415-20. [MEDLINE: 7471]

\section{Glasgow 1995 \{published data only\}}

Glasgow RE, Toobert DJ, Hampson SE, Noell JW. A brief office-based intervention to facilitate diabetes dietary self-

Telephone follow-up, initiated by a hospital-based health professional, for postdischarge problems in patients discharged from hospital 


\section{Greineder 1999 \{published data only\}}

Greineder DK, Loane KC, Parks P. A randomized controlled trial of a pediatric asthma outreach program. Journal of Allergy and Clinical Immunology 1999;103(3):436-40. [MEDLINE: 13452]

\section{Griffin 1989 \{published data only\}}

Griffin KW, McNabb WL, Shields MC. Telephone instruction as an adjunct to patient education of children with asthma. Journal of Healthcare Education and Training 1989;4(1):1-6. [MEDLINE: 9366]

\section{Grunfeld 1999 \{published data only\}}

Grunfeld E, Gray A, Mant D, Yudkin P, Adewuyi DR, Coyle D, et al. Follow up of breast cancer in primary care vs specialist care: results of an economic evaluation. British Journal of Cancer 1999;79(7-8):1227-33. [MEDLINE: 13569]

\section{Gulliford 1997 \{published data only\}}

Gulliford T, Opomu M, Wilson E, Hanham I, Epstein R. Popularity of less frequent follow up for breast cancer in randomised study: initial findings from the hotline study. BMJ 1997;314(7075):174-7. [MEDLINE: 6552]

\section{Harrison 1999 \{published data only\}}

Harrison R, Clayton W, Wallace P. Virtual outreach: a telemedicine pilot study using a cluster-randomized controlled design. Journal of Telemedicine and Telecare 1999;5(2):126-30. [MEDLINE: 4408]

\section{Hartmann 1996 \{published data only\}}

Hartmann DJ, Sullivan WP. Residential crisis services as an alternative to inpatient care. Families in Society 1996;77(8):496-501. [MEDLINE: 13901]

\section{Hasseler 2002 \{published data only\}}

Hasseler M. Inpatient postpartum nursing: evaluation of "holistic" and "traditional" nursing processes in the post partum period. Pflege 2002;15(5):170-80. [MEDLINE: 1481]

\section{Hauber 2002 \{published data only\}}

Hauber RP, Jones ML. Telerehabilitation support for families at home caring for individuals in prolonged states of reduced consciousness. Journal of Head Trauma Rehabilitation 2002;17(6):535-41. [MEDLINE: 1029]

\section{Hayes 2001a \{published data only\}}

Hayes A, Buffum M. Educating patients after conscious sedation for gastrointestinal procedures. Gastroenterology Nursing 2001;24(2):54-7. [MEDLINE: 2476]

\section{Hayes 2001b \{published data only\}}

Hayes JT, Boucher JL, Pronk NP, Gehling E, Spencer M, Waslaski J. The role of the certified diabetes educator in telephone counseling. The Diabetes Educator 2001;27(3):377-86. [MEDLINE: 2368]

\section{Heidenreich 1999 \{published data only\}}

Heidenreich PA, Ruggerio CM, Massie BM. Effect of a home monitoring system on hospitalization and resource use for patients with heart failure. American Heart Journal 1999;138(4 Pt 1):633-40. [MEDLINE: 4637]
Heller 1993 \{published data only\}

Heller RF, Knapp JC, Valenti LA, Dobson AJ. Secondary prevention after acute myocardial infarction. American Journal of Cardiology 1993;72(11):759-62. [MEDLINE: 8120]

Hendricks 2000 \{published data only\}

Hendricks LE, Hendricks RT. The effect of diabetes selfmanagement education with frequent follow-up on the health outcomes of African American men. The Diabetes Educator 2000;26(6):995-1002. [MEDLINE: 2366]

\section{Hernandez 2003 \{published data only\}}

Hernandez C, Casas A, Escarrabill J, Alonso J, Puig-Junoy J, Farrero E, et al. Home hospitalisation of exacerbated chronic obstructive pulmonary disease patients. European Respiratory Journal 2003;21(1):58-67. [MEDLINE: 69]

Hickey 1996 \{published data only\}

Hickey ML, Kleefield SF, Pearson SD, Hassan SM, Harding M, Haughie $P$, et al. Payer-hospital collaboration to improve patient satisfaction with hospital discharge. The Joint Commission Journal on Quality Improvement 1996;22(5):336-44. [MEDLINE: 6960]

Higgins 2001 \{published data only\}

Higgins HC, Hayes RL, McKenna KT. Rehabilitation outcomes following percutaneous coronary interventions (PCI). Patient Education \& Counseling 2001;43(3):219-30. [MEDLINE: 338]

Hillebrand 1996 \{published data only\}

Hillebrand T, Wirth A. Evaluation of an outpatient care program for obese patients after an inpatient treatment. [German]. Pravention und Rehabilitation 1996;8(2):83-7. [MEDLINE: 763]

Hornblow 1980 \{published data only\}

Hornblow AR, Sloane HR. Evaluating the effectiveness of a telephone counselling service. British Journal of Psychiatry 1980;137:377-8. [MEDLINE: 10117]

\section{Hoskins 1985 \{published data only\}}

Hoskins P, Alford J, Fowler P, Bolton T, Pech C, Hosking M, et al. Outpatient stabilization programme: an innovative approach in the management of diabetes. Diabetes Research 1985;2(2):85-8. [MEDLINE: 9825]

\section{Hoskins 2001 \{published data only\}}

Hoskins CN, Haber J, Budin WC, Cartwright-Alcarese F, Kowalski MO, Panke J, Maislin G. Breast cancer: education, counseling, and adjustment: a pilot study. Psychological Reports 2001;89(3):677-704. [MEDLINE: 2517]

\section{Houzard 1998 \{published data only\}}

Houzard S, Pateron D, Bleichner G, Dabrowski G, Davido A, Grange $P$, et al. Outpatient care in emergency departments: feasibility and comparison of three strategies for followup. European Journal of Emergency Medicine 1998;5:335-9. [MEDLINE: 12686]

\section{Hui 2000 \{published data only\}}

Hui DS, Chan JK, Choy DK, Ko FW, Li TS, Leung RC, Lai CK. Effects of augmented continuous positive airway pressure

Telephone follow-up, initiated by a hospital-based health professional, for postdischarge problems in patients discharged from hospital 
education and support on compliance and outcome in a Chinese population. Chest 2000;117(5):1410-6. [MEDLINE: 4124]

\section{Intagliata 1976 \{published data only\}}

Intagliata J. A telephone follow-up procedure for increasing the effectiveness of a treatment program for alcoholics. Journal of Studies on Alcohol 1976;37(9):1330-5. [MEDLINE: 10251]

\section{Jahanshahi 1994 \{published data only\}}

Jahanshahi M, Brown RG, Whitehouse C, Quinn N, Marsden CD. Contact with a nurse practitioner: A short-term evaluation study in Parkinson's disease and dystonia. Behavioural Neurology 1994;7:189-96. [MEDLINE: 13020]

\section{James 1994 \{published data only\}}

James ND, Guerrero D, Brada M. Who should follow up cancer patients? Nurse specialist based outpatient care and the introduction of a phone clinic system. Clinical oncology (Royal College of Radiologists (Great Britain)) 1994;6(5):283-7. [MEDLINE: 8018]

\section{Joffe 1995 \{published data only\}}

Joffe GM, Symonds R, Alverson D, Chilton L. The effect of a comprehensive prematurity prevention program on the number of admissions to the neonatal intensive care unit. Journal of Perinatology 1995;15(4):305-9. [MEDLINE: 7356]

\section{Johnson 2000a \{published data only\}} Johnson BF, Hamilton G, Fink J, Lucey G, Bennet N, Lew R. A design for testing interventions to improve adherence within a hypertension clinical trial. Controlled Clinical Trials 2000;21(1):62-72. [MEDLINE: 4354]

\section{Johnson 2000b \{published data only\}}

Johnson JL, Ratner PA, Bottorff JL, Hall W, Dahinten S. Preventing smoking relapse in postpartum women. Nursing Research 2000;49(1):44-52. [MEDLINE: 4344]

\section{Johnson 2000c \{published data only\}}

Johnson K. Use of telephone follow-up for post-cardiac surgery patients. Intensive and Critical Care Nursing 2000;16(3):144-50. [MEDLINE: 4031]

\section{Jolly 2003 \{published data only\}}

Jolly K, Lip G, Sandercock J, Greenfield S, Raftery J, Mant J, et al. Home-based versus hospital-based cardiac rehabilitation after myocardial infarction or revascularisation: design and rationale of the Birmingham Rehabilitation Uptake Maximisation Study (BRUM): a randomised controlled trial [ISRCTN72884263]. BMC Cardiovascular Disorders 2003;3(1):10. [MEDLINE: 15353]

\section{Jones 1988b \{published data only\}}

Jones J, Clark W, Bradford J, Dougherty J. Efficacy of a telephone follow-up system in the emergency department. Journal of Emergency Medicine 1988;6(3):249-54. [MEDLINE: 9448]

\section{Jones 1997 \{published data only\}}

Jones JS, Young MS, LaFleur RA, Brown MD. Effectiveness of an organized follow-up system for elder patients released from the emergency department. Academic Emergency Medicine 1997;4(12):1147-52. [MEDLINE: 6064]

Jowers 2000 \{published data only\}

Jowers JR, Corsello PR, Shafer AL, Schwartz A, Tinkelman DG. Partnering specialist care with nurse case management: a pilot project for asthma. Journal of Clinical Outcomes Management 2000;7(5):17-22. [MEDLINE: 10815]

\section{Kasper 2002 \{published data only\}}

Kasper EK, Gerstenblith G, Hefter G, Van Anden E, Brinker JA, Thiemann DR, et al. A randomized trial of the efficacy of multidisciplinary care in heart failure outpatients at high risk of hospital readmission. Journal of the American College of Cardiology 2002;39(3):471-80. [MEDLINE: 2520]

Kelly 1999 \{published data only\}

Kelly DF, Faught WJ, Holmes LA. Ovarian cancer treatment: the benefit of patient telephone follow-up post-chemotherapy. Canadian Oncology Nursing Journal 1999;9(4):175-8. [MEDLINE: 4165]

\section{King 1991 \{published data only\}}

King $\mathrm{H}$. A telephone reassurance service: a natural support system for the elderly. Journal of Gerontological Social Work 1991;16(1-2):159-77. [MEDLINE: 15192]

\section{Kirkman 1994 \{published data only\}}

Kirkman MS, Weinberger M, Landsman PB, Samsa GP, Shortliffe EA, Simel DL, Feussner JR. A telephone-delivered intervention for patients with NIDDM. Effect on coronary risk factors. Diabetes Care 1994;17(8):840-6. [MEDLINE: 7768]

\section{Kirscht 1981 \{published data only\}}

Kirscht JP, Kirscht JL, Rosenstock IM. A test of interventions to increase adherence to hypertensive medical regimens. Health Education Quarterly 1981;8(3):261-72. [MEDLINE: 10107]

\section{Kokubu 1999 \{published data only\}}

Kokubu F, Suzuki H, Sano Y, Kihara N, Adachi M. Telemedicine system for high-risk asthmatic patients. Arerugi 1999;48(7):700-12. [MEDLINE: 4672]

\section{Kokubu 2000 \{published data only\}}

Kokubu F, Nakajima S, Ito K, Makino S, Kitamura S, Fukuchi Y, et al. Hospitalization reduction by an asthma tele-medicine system. Arerugi 2000;49(1):19-31. [MEDLINE: 4284]

\section{Korner-Bitensky 1994 \{published data only\}}

Korner-Bitensky N, Wood-Dauphinee S, Siemiatycki J, Shapiro S, Becker R. Health-related information postdischarge: telephone versus face-to-face interviewing. Archives of Physical Medicine and Rehabilitation 1994;75(12):1287-96. [MEDLINE: 7628]

\section{Kramer 2003 \{published data only\}}

Kramer JF, Speechley M, Bourne R, Rorabeck C, Vaz M. Comparison of clinic- and home-based rehabilitation programs after total knee arthroplasty. Clinical Orthopaedics and Related Research 2003, (410):225-34. [MEDLINE: 1050]

Telephone follow-up, initiated by a hospital-based health professional, for postdischarge problems in patients discharged from hospital 
Kunik 2001 \{published data only\}

Kunik ME, Braun U, Stanley MA, Wristers K, Molinari V, Stoebner D, Orengo CA. One session cognitive behavioural therapy for elderly patients with chronic obstructive pulmonary disease. Psychological Medicine 2001;31(4):717-23. [MEDLINE: 3242]

\section{Lando 2001 \{published data only\}}

Lando HA, Valanis BG, Lichtenstein E, Curry SJ, McBride CM, Pirie PL, Grothaus LC. Promoting smoking abstinence in pregnant and postpartum patients: a comparison of 2 approaches. American Journal of Managed Care 2001;7(7):685-93. [MEDLINE: 3014]

\section{Laramee 2003 \{published data only\}}

Laramee AS, Levinsky SK, Sargent J, Ross R, Callas P. Case management in a heterogeneous congestive heart failure population: a randomized controlled trial. Archives of Internal Medicine 2003;163(7):809-17. [MEDLINE: 1136]

\section{Lear 2001 \{published data only\}}

Lear SA, Ignaszewski A, Laquer EA, Pritchard PH, Frohlich JJ. Extensive lifestyle management intervention following cardiac rehabilitation: pilot study. Rehabilitation Nursing 2001;26(6):227-32. [MEDLINE: 2193]

\section{Lear 2002 \{published data only\}}

Lear SA, Ignaszewski A, Linden W, Brozic A, Kiess M, Spinelli JJ, et al. A randomized controlled trial of an extensive lifestyle management intervention (ELMI) following cardiac rehabilitation: study design and baseline data. Current Controlled Trials in Cardiovascular Medicine 2002;3(1):9. [MEDLINE: 1464]

\section{Lee 1999 \{published data only\}}

Lee M, Kemp JA, Canning A, Egan C, Tataronis G, Farraye FA. A randomized controlled trial of an enhanced patient compliance program for Helicobacter pylori therapy. Archives of Internal Medicine 1999;159(19):2312-6. [MEDLINE: 4559]

\section{Liew 1994 \{published data only\}}

Liew SC, Huber D, Jeffs C. Day-only admission for varicose vein surgery. Australian and New Zealand Journal of Surgery 1994;64(10):688-91. [MEDLINE: 7691]

\section{Litzelman 1993 \{published data only\}}

Litzelman DK, Slemenda CW, Langefeld CD, Hays LM, Welch MA, Bild DE, et al. Reduction of lower extremity clinical abnormalities in patients with non-insulin-dependent diabetes mellitus: a randomized, controlled trial. Annals of Internal Medicine 1993;119(1):36-41. [MEDLINE: 8176]

\section{Lundblad 2001 \{published data only\}}

Lundblad B, Byrne MW, Helstrom A. International pediatric nursing. Continuing nursing care needs of children at time of discharge from one regional medical center in Sweden. Journal of Pediatric Nursing: Nursing Care of Children and Families 2001;16(1):73-8. [MEDLINE: 10844]

\section{Lynch 2003 \{published data only\}}

Lynch TR, Morse JQ, Mendelson T, Robins CJ. Dialectical behavior therapy for depressed older adults: a randomized pilot study. American Journal of Geriatric Psychiatry 2003;11(1):33-45. [MEDLINE: 1371]

\section{MacMahon 1999 \{published data only\}}

MacMahon DG. Parkinson's disease nurse specialists: an important role in disease management. Neurology 1999;52(7 Suppl 3):S21-S25. [MEDLINE: 5005]

\section{Madge 1997 \{published data only\}}

Madge P, McColl J, Paton J. Impact of a nurse-led home management training programme in children admitted to hospital with acute asthma: a randomised controlled study. Thorax 1997;52(3):223-8. [MEDLINE: 6477]

Madonna 1999 \{published data only\}

Madonna MG, Keating MM. Multiple sclerosis pathways: an innovative nursing role in disease management. Journal of Neuroscience Nursing 1999;31(6):332-5. [MEDLINE: 4259]

\section{Maiman 1979 \{published data only\}}

Maiman L, Green L, Gibson G, MacKenzie E. Education for selftreatment by adult asthmatics. JAMA 1979;241(18):1919-22. [MEDLINE: 13377]

\section{Maisiak 1996a \{published data only\}}

Maisiak R, Austin J, Heck L. Health outcomes of two telephone interventions for patients with rheumatoid arthritis or osteoarthritis. Arthritis and Rheumatism 1996;39(8):1391-9. [MEDLINE: 6850]

\section{Maisiak 1996b \{published data only\}}

Maisiak R, Austin JS, West SG, Heck L. The effect of personcentered counseling on the psychological status of persons with systemic lupus erythematosus or rheumatoid arthritis: a randomized, controlled trial. Arthritis Care \& Research 1996;9(1):60-6. [MEDLINE: 7056]

\section{Manian 1993 \{published data only\}}

Manian FA, Meyer L. Comparison of patient telephone survey with traditional surveillance and monthly physician questionnaires in monitoring surgical wound infections. Infection Control and Hospital Epidemiology 1993;14(4):216-8. [MEDLINE: 10434]

\section{Marcus 1998 \{published data only\}}

Marcus AC, Garrett KM, Cella D, Wenzel LB, Brady MJ, Crane LA, et al. Telephone counseling of breast cancer patients after treatment: a description of a randomized clinical trial. PsychoOncology 1998;7(6):470-82. [MEDLINE: 595]

\section{Marrero 1995 \{published data only\}}

Marrero DG, Vandagriff JL, Kronz K, Fineberg NS, Golden MP, Gray D, et al. Using telecommunication technology to manage children with diabetes: the Computer-Linked Outpatient Clinic (CLOC) study. The Diabetes Educator 1995;21(4):313-9. [MEDLINE: 7367] 
Mason 1998 \{published data only\}

Mason L. Day surgery: improving care through follow up contact. British Journal of Theatre Nursing 1998;8(6):11-3. [MEDLINE: 10573]

\section{Maunsell 1996 \{published data only\}}

Maunsell E, Brisson J, Deschenes L, Frasure-Smith N. Randomized trial of a psychologic distress screening program after breast cancer: effects on quality of life. Journal of Clinical Oncology 1996;14(10):2747-55. [MEDLINE: 6764]

\section{McCorkle 2000 \{published data only\}}

McCorkle R, Strumpf NE, Nuamah IF, Adler DC, Cooley ME, Jepson C, et al. A specialized home care intervention improves survival among older post-surgical cancer patients. Journal of the American Geriatrics Society 2000;48(12):1707-13. [MEDLINE: 3608]

\section{McDonald 2002 \{published data only\}}

McDonald K, Ledwidge M, Cahill J, Quigley P, Maurer B, Travers B, et al. Heart failure management: multidisciplinary care has intrinsic benefit above the optimization of medical care. Journal of Cardiac Failure 2002;8(3):142-8. [MEDLINE: 2027]

\section{McGrath 2002 \{published data only\}}

McGrath P. Allied health professional services for oncology outpatients: an Australian comparative study. Journal of Allied Health 2002;31(1):29-34. [MEDLINE: 2378]

\section{McIntosh 1994 \{published data only\}}

McIntosh J, Worley N. Beyond discharge: telephone follow-up and aftercare. Journal of Psychosocial Nursing and Mental Health Services 1994;32(10):21-7. [MEDLINE: 7704]

\section{McMurray 1998 \{published data only\}}

McMurray JJ, Stewart S. Nurse led, multidisciplinary intervention in chronic heart failure. Heart 1998;80(5):430-1. [MEDLINE: 5218]

\section{McNamara 1995 \{published data only\}}

McNamara KJ. Patients leaving the ED without being seen by a physician: is same-day follow-up indicated?. American Journal of Emergency Medicine 1995;13(2):136-41. [MEDLINE: 818]

\section{Meenan 1998 \{published data only\}}

Meenan RT, Stevens VJ, Hornbrook MC, La Chance PA, Glasgow RE, Hollis JF, et al. Cost-effectiveness of a hospitalbased smoking cessation intervention. Medical Care 1998;36:670-8. [MEDLINE: 12643]

Miller 1995 \{published data only\}

Miller NH, Smith PM, Taylor CB, Sobel D, DeBusk RF. Smoking cessation in hospitalized patients - results of a randomized trial.. Circulation 1995;92(8SS):179. [MEDLINE: 15344]

\section{Miller 1997a \{published data only\}}

Miller NH, Smith PM, DeBusk RF, Sobel DS, Taylor CB. Smoking cessation in hospitalized patients. Results of a randomized trial. Archives of Internal Medicine 1997;157(4):409-15. [MEDLINE: 6494]

\section{Miller 1997b \{published data only\}}

Miller NH. How to achieve smoking cessation among hospitilized patients. Consultant 1997;37(6):1653. [MEDLINE: 15292]

\section{Miller 2002a \{published data only\}}

Miller L, Weissman M. Interpersonal psychotherapy delivered over the telephone to recurrent depressives. A pilot study. Depression and Anxiety 2002;16(3):114-7. [MEDLINE: 1566]

Miller 2002b \{published data only\}

Miller L, Caton S, Lynch D. Telephone clinic improves quality of follow-up care for chronic bowel disease. Nursing Times 2002;98(31):36-8. [MEDLINE: 11050]

\section{Miranda 2002 \{published data only\}}

Miranda MB, Gorski LA, LeFevre JG, Levac KA, Niederstadt JA, Toy AL. An evidence-based approach to improving care of patients with heart failure across the continuum. Journal of Nursing Care Quality 2002;17(1):1-14. [MEDLINE: 14113]

Mishel 2002 \{published data only\}

Mishel MH, Belyea M, Germino BB, Stewart JL, Bailey DE, Jr, Robertson C, Mohler J. Helping patients with localized prostate carcinoma manage uncertainty and treatment side effects: nurse-delivered psychoeducational intervention over the telephone. Cancer 2002;94(6):1854-66. [MEDLINE: 2355]

\section{Mohlman 2003 \{published data only\}}

Mohlman J, Gorenstein EE, Kleber M, de Jesus M, Gorman JM, Papp LA. Standard and enhanced cognitive-behavior therapy for late-life generalized anxiety disorder: two pilot investigations. American Journal of Geriatric Psychiatry 2003;11(1):24-32. [MEDLINE: 1372]

\section{Mohr 2000 \{published data only\}}

Mohr DC, Likosky W, Bertagnolli A, Goodkin DE, Van Der WJ, Dwyer P, Dick LP. Telephone-administered cognitive-behavioral therapy for the treatment of depressive symptoms in multiple sclerosis. Journal of Consulting and Clinical Psychology 2000;68(2):356-61. [MEDLINE: 4170]

Moran 1998 \{published data only\}

Moran SJ, Jarvis S, Ewings P, Parkin FA. It's good to talk, but is it effective? A comparative study of telephone support following day surgery... including commentary by Needham $\mathrm{Y}$ and Rendell J with author response. Clinical Effectiveness in Nursing 1998;2(4):175-84. [MEDLINE: 10534]

\section{Morrison 2001 \{published data only\}}

Morrison J, Bergauer NK, Jacques D, Coleman SK, Stanziano GJ. Telemedicine: cost-effective management of high-risk pregnancy. Managed Care 2001;10(11):42-9. [MEDLINE: 2619]

\section{Napolitano 2002 \{published data only\}}

Napolitano MA, Babyak MA, Palmer S, Tapson V, Davis RD, Blumenthal JA. Effects of a telephone-based psychosocial intervention for patients awaiting lung transplantation. Chest 2002;122(4):1176-84. [MEDLINE: 1631]

Telephone follow-up, initiated by a hospital-based health professional, for postdischarge problems in patients discharged from hospital 
Naylor 1999 \{published data only\}

Naylor MD, McCauley KM. The effects of a discharge planning and home follow-up intervention on elders hospitalized with common medical and surgical cardiac conditions. Journal of Cardiovascular Nursing 1999;14(1):44-54. [MEDLINE: 4587]

Nelson 2001 \{published data only\}

* Nelson JR. The importance of postdischarge telephone follow-up for hospitalists: a view from the trenches. American Journal of Medicine 2001;111(9B):43S-44S. [MEDLINE: 2571]

Nelson JR. The importance of postdischarge telephone followup for hospitalists: a view from the trenches. Disease-A-Month 2002;48(4):273-5.

\section{Newman 2002 \{published data only\}}

Newman M. A specialist nurse intervention reduced hospital readmissions in patients with chronic heart failure. Evidence Based Nursing 2002;5(2):55. [MEDLINE: 11074]

Nicklin 1986 \{published data only\}

Nicklin WM. Postdischarge concerns of cardiac patients as presented via a telephone callback system. Heart \& Lung: Journal of Critical Care 1986;15(3):268-72. [MEDLINE: 9692]

Nijdam 1999 \{published data only\}

Nijdam W, Pool A, Koorenaar C. Het telefonisch verpleegkundig follow-up spreekuur bij patiënten met hersenmetastasen die zijn behandeld met radiotherapie. Verpleegkunde 1999;14(4):271-2. [MEDLINE: 15220]

\section{Northouse 2002 \{published data only\}}

Northouse LL, Walker J, Schafenacker A, Mood D, Mellon S, Galvin E, et al. A family-based program of care for women with recurrent breast cancer and their family members. Oncology Nursing Forum 2002;29(10):1411-9. [MEDLINE: 1539]

\section{O'Neill 2001 \{published data only\}}

O'Neill K, Silvestri A, McDaniel-Yakscoe N. A pediatric emergency department follow-up system: completing the cycle of care. Pediatric Emergency Care 2001;17(5):392-5. [MEDLINE: 2764]

\section{Oddone 1999 \{published data only\}}

Oddone EZ, Weinberger M, Giobbie-Hurder A, Landsman P, Henderson W. Enhanced access to primary care for patients with congestive heart failure. Veterans Affairs Cooperative Study Group on Primary Care and Hospital Readmission. Effective Clinical Practice 1999;2(5):201-9. [MEDLINE: 4425]

\section{Oh 2003 \{published data only\}}

Oh JA, Kim HS, Yoon KH, Choi ES. A telephone-delivered intervention to improve glycemic control in type 2 diabetic patients. Yonsei Medical Journal 2003;44(1):1-8. [MEDLINE: 1227]

Pal 1998 \{published data only\}

Pal B. Following up outpatients by telephone: pilot study. BMJ 1998;316(7145):1647. [MEDLINE: 10610]
Pal 2001 \{published data only\}

Pal B. Tele-rheumatology: telephone follow up and cyberclinic. Computer Methods \& Programs in Biomedicine 2001;64(3):189-195. [MEDLINE: 15246]

Palmer 2001 \{published data only\}

Palmer HC, Armistead NS, Elnicki DM, Halperin AK, Ogershok PR, Manivannan S, et al. The effect of a hospitalist service with nurse discharge planner on patient care in an academic teaching hospital. American Journal of Medicine 2001;111(8):627-32. [MEDLINE: 14206]

Palmer 2002 \{published data only\}

Palmer RL, Birchall H, McGrain L, Sullivan V. Self-help for bulimic disorders: a randomised controlled trial comparing minimal guidance with face-to-face or telephone guidance. British Journal of Psychiatry 2002;181:230-5. [MEDLINE: 1924]

Peterson 2002 \{published data only\}

Peterson GM. Practical utility of case-management telephone intervention in heart failure?. Archives of Internal Medicine 2002;162(18):2142-3. [MEDLINE: 1637]

Pidd 2000 \{published data only\} Pidd H, McGrory KJ, Payne SR. Telephone follow-up after urological surgery. Professional Nurse 2000;15(7):449-51. [MEDLINE: 3607]

Poncia 2000 \{published data only\}

Poncia HDM, Ryan J, Carver M. Next day telephone follow up of the elderly: a needs assessment and critical incident monitoring tool for the accident and emergency department. Journal of Accident \& Emergency Medicine 2000;17(5):337-40. [MEDLINE: 14462]

\section{Powell 2001 \{published data only\}}

Powell P, Bentall RP, Nye FJ, Edwards RH. Randomised controlled trial of patient education to encourage graded exercise in chronic fatigue syndrome. BMJ 2001;322(7283):387-90. [MEDLINE: 3531]

\section{Powers 1983 \{published data only\}}

Powers DA, Hamilton CW, Roberts KB. Pharmacist intervention in methadone administration to cancer patients with chronic pain. American Journal of Hospital Pharmacy 1983;40:1520-3. [MEDLINE: 13364]

\section{Proctor 2000 \{published data only\}}

Proctor EK, Morrow-Howell N, Li H, Dore P. Adequacy of home care and hospital readmission for elderly congestive heart failure patients. Health and Social Work 2000;25(2):87-96. [MEDLINE: 4063]

\section{Pugh 1999 \{published data only\}}

Pugh LC, Tringali RA, Boehmer J, Blaha C, Kruger NR, Capauna TA, et al. Partners in care: a model of collaboration. Holistic Nursing Practice 1999;13(2):61-5. [MEDLINE: 5074]

\section{Racelis 1998 \{published data only\}}

Racelis MC, Lombardo K, Verdin J. Impact of telephone reinforcement of risk reduction education on patient

Telephone follow-up, initiated by a hospital-based health professional, for postdischarge problems in patients discharged from hospital 
compliance. Journal of Vascular Nursing 1998;16(1):16-20. [MEDLINE: 5450]

\section{Rakowski 1994 \{published data only\}}

Rakowski J. An emergency department telephone follow-up service. Journal of Emergency Nursing 1994;20(3):199-203. [MEDLINE: 15325]

\section{Rauh 1999 \{published data only\}}

Rauh RA, Schwabauer NJ, Enger EL, Moran JF. A community hospital-based congestive heart failure program: impact on length of stay, admission and readmission rates, and cost. American Journal of Managed Care 1999;5(1):37-43. [MEDLINE: 13429]

\section{Rawl 1998 \{published data only\}}

Rawl SM, Easton KL, Kwiatkowski S, Zemen D, Burczyk B. Effectiveness of a nurse-managed follow-up program for rehabilitation patients after discharge. Rehabilitation Nursing 1998;23(4):204-9. [MEDLINE: 5334]

\section{Rawl 2002 \{published data only\}}

Rawl SM, Given BA, Given CW, Champion VL, Kozachik SL, Kozachik SL, et al. Intervention to improve psychological functioning for newly diagnosed patients with cancer. Oncology Nursing Forum 2002;29(6):967-75. [MEDLINE: 2099]

\section{Rene 1992 \{published data only\}}

Rene J, Weinberger M, Mazzuca SA, Brandt KD, Katz BP. Reduction of joint pain in patients with knee osteoarthritis who have received monthly telephone calls from lay personnel and whose medical treatment regimens have remained stable. Arthritis and Rheumatism 1992;35(5):511-5. [MEDLINE: 8565]

\section{Rich 1995 \{published data only\}}

Rich MW, Beckham V, Wittenberg C, Leven CL, Freedland KE, Carney RM. A multidisciplinary intervention to prevent the readmission of elderly patients with congestive heart failure. New England Journal of Medicine 1995;333(18):1190-5. [MEDLINE: 13444]

\section{Riegel 1996 \{published data only\}}

Riegel B, Gates DM, Gocka I, Medina L, Odell C, Rich M, Finken JS. Effectiveness of a program of early hospital discharge of cardiac surgery patients [corrected] [published erratum appears in J CARDIOVASC NURS 1997 Apr; 11(3): viii]. Journal of Cardiovascular Nursing 1996;11(1):63-75. [MEDLINE: 10331]

\section{Riegel 2000 \{published data only\}}

Riegel B, Carlson B, Glaser D, Hoagland P. Which patients with heart failure respond best to multidisciplinary disease management?. Journal of Cardiac Failure 2000;6(4):290-9. [MEDLINE: 13669]

\section{Rieger 1995 \{published data only\}}

Rieger ID, Henderson-Smart DJ. A neonatal early discharge and home support programme: shifting care into the community. Journal of paediatrics and child health 1995;31(1):33-7. [MEDLINE: 13445]
Ries 2003 \{published data only\}

Ries AL, Kaplan RM, Myers R, Prewitt LM. Maintenance after pulmonary rehabilitation in chronic lung disease: a randomized trial. American Journal of Respiratory and Critical Care Medicine 2003;167(6):880-8. [MEDLINE: 1413]

Rigotti 1997 \{published data only\}

Rigotti NA, Arnsten JH, McKool KM, Wood-Reid KM, Pasternak RC, Singer DE. Efficacy of a smoking cessation program for hospital patients. Archives of Internal Medicine 1997;157(22):2653-60. [MEDLINE: 669]

Riley 1989 \{published data only\}

Riley J. Telephone call-backs: final patient care evaluation. Nursing Management 1989;20(9):64-6. [MEDLINE: 9228]

Roberts 1995 \{published data only\}

Roberts J, Browne GB, Streiner D, Gafni A, Pallister R, Hoxby H, et al. Problem-solving counselling or phone-call support for outpatients with chronic illness: effective for whom?. Canadian Journal of Nursing Research 1995;27(3):111-37. [MEDLINE: 7574]

Roglieri 1997 \{published data only\}

Roglieri JL, Futterman R, McDonough KL, Malya G, Karwath KR, Bowman $\mathrm{D}$, et al. Disease management interventions to improve outcomes in congestive heart failure. American Journal of Managed Care 1997;3(12):1831-9. [MEDLINE: 10574]

Romano 2001 \{published data only\}

Romano MJ, Hernandez J, Gaylor A, Howard S, Knox R. Improvement in asthma symptoms and quality of life in pediatric patients through specialty care delivered via telemedicine. Telemedicine Journal and e-Health 2001;7(4):281-6. [MEDLINE: 2416]

Rosbe 2000 \{published data only\}

Rosbe KW, Jones D, Jalisi S, Bray MA. Efficacy of postoperative follow-up telephone calls for patients who underwent adenotonsillectomy. Archives of Otolaryngology -- Head \& Neck Surgery 2000;126(6):718-21. [MEDLINE: 481]

Rosswurm 1998 \{published data only\}

Rosswurm MA, Lanham DM. Discharge planning for elderly patients. Journal of Gerontological Nursing 1998;24(5):14-21. [MEDLINE: 5490]

\section{Ruchlin 2001 \{published data only\}}

Ruchlin HS, Elkin EB, Allegrante JP. The economic impact of a multifactorial intervention to improve postoperative rehabilitation of hip fracture patients. Arthritis and Rheumatism 2001;45(5):446-52. [MEDLINE: 2777]

\section{Sanders 1997 \{published data only\}}

Sanders D, Bass C, Mayou RA, Goodwin S, Bryant BM, Tyndel S. Non-cardiac chest pain: why was a brief intervention apparently ineffective?. Psychological Medicine 1997;27(5):1033-40. [MEDLINE: 6224] 
Sandgren 2000 \{published data only\}

Sandgren AK, McCaul KD, King B, O'Donnell S, Foreman G. Telephone therapy for patients with breast cancer. Oncology Nursing Forum 2000;27(4):683-8. [MEDLINE: 4078]

\section{Sandgren 2003 \{published data only\}}

Sandgren AK, McCaul KD. Short-term effects of telephone therapy for breast cancer patients. Health Psychology 2003;22(3):310-5. [MEDLINE: 1035]

\section{Sardell 2000 \{published data only\}}

Sardell S, Sharpe G, Ashley S, Guerrero D, Brada M. Evaluation of a nurse-led telephone clinic in the follow-up of patients with malignant glioma. Clinical oncology (Royal College of Radiologists (Great Britain)) 2000;12(1):36-41. [MEDLINE: 4223]

\section{Schatz 2003 \{published data only\}}

Schatz M, Rodriguez E, Falkoff R, Zeiger RS. The relationship of frequency of follow-up visits to asthma outcomes in patients with moderate persistent asthma. Journal of Asthma 2003;40(1):49-53. [MEDLINE: 1128]

\section{Schectman 1994 \{published data only\}}

Schectman G, Hiatt J, Hartz A. Telephone contacts do not improve adherence to niacin or bile acid sequestrant therapy. Annals of Pharmacotherapy 1994;28(1):29-35. [MEDLINE: 7995]

\section{Schultz 1993 \{published data only\}}

Schultz SJ. Educational and behavioral strategies related to knowledge of and participation in an exercise program after cardiac positron emission tomography. Patient Education \& Counseling 1993;22(1):47-57. [MEDLINE: 8091]

\section{Sciamanna 2000 \{published data only\}}

Sciamanna CN, Stillman FA, Hoch JS, Butler JH, Gass KG, Ford DE. Opportunities for improving inpatient smoking cessation programs: a community hospital experience. Preventive Medicine 2000;30(6):496-503. [MEDLINE: 3959]

\section{Shah 1998 \{published data only\}}

Shah NB, Der E, Ruggerio C, Heidenreich PA, Massie BM. Prevention of hospitalizations for heart failure with an interactive home monitoring program. American Heart Journal 1998;135(3):373-8. [MEDLINE: 649]

\section{Shapiro 1995 \{published data only\}}

Shapiro C. Shortened hospital stay for low-birth-weight infants: nuts and bolts of a nursing intervention project. Journal of Obstetric, Gynecologic, and Neonatal Nursing 1995;24(1):56-62. [MEDLINE: 7602]

\section{Shon 2002 \{published data only\}}

Shon KH, Park SS. Medication and symptom management education program for the rehabilitation of psychiatric patients in Korea: the effects of promoting schedule on self-efficacy theory. Yonsei Medical Journal 2002;43(5):579-89. [MEDLINE: 1585]

\section{Shu 1996 \{published data only\}}

Shu E, Mirmina Z, Nystrom K. A telephone reassurance program for elderly home care clients after discharge. Home Healthcare Nurse 1996;14(3):154-61. [MEDLINE: 7031]

\section{Siegel 1992 \{published data only\}}

Siegel K, Mesagno FP, Karus DG, Christ G. Reducing the prevalence of unmet needs for concrete services of patients with cancer: evaluation of a computerized telephone outreach system. Cancer 1992;69(7):1873-83. [MEDLINE: 8585]

\section{Simon 1997 \{published data only\}}

Simon JA, Solkowitz SN, Carmody TP, Browner WS. Smoking cessation after surgery: a randomized trial. Archives of Internal Medicine 1997;157(12):1371-6. [MEDLINE: 6303]

\section{Simon 2003 \{published data only\}}

Simon JA, Carmody TP, Hudes ES, Snyder E, Murray J. Intensive smoking cessation counseling versus minimal counseling among hospitalized smokers treated with transdermal nicotine replacement: a randomized trial. American Journal of Medicine 2003;114(7):555-62. [MEDLINE: 1063]

Sluijk 1998 \{published data only\}

Sluijk C, Elzinga A. Afdelingsverpleegkundigen garanderen continuiteit van zorg: een telefonisch verpleegkundig spreekuur. TVZ 1998;108(8):252-4. [MEDLINE: 15226]

Smeenk 1998a \{published data only\}

Smeenk FW, van Haastregt JC, Gubbels EM, de Witte LP, Crebolder HF. Care process and satisfaction analysis of a transmural home care program. International Journal of Nursing Studies 1998;35(3):146-54. [MEDLINE: 5410]

Smeenk 1998b \{published data only\}

Smeenk FW, Ament AJ, van Haastregt J, de Witte LP, Crebolder $\mathrm{H}$. Cost analysis of transmural home care for terminal cancer patients. Patient Education \& Counseling 1998;35(3):201-11. [MEDLINE: 13497]

Smith 1988 \{published data only\}

Smith DM, Weinberger M, Katz BP, Moore PS. Postdischarge care and readmissions. Medical Care 1988;26(7):699-708. [MEDLINE: 9421]

\section{Smith 2002 \{published data only\}}

Smith PM, Reilly KR, Miller NH, DeBusk RF, Taylor CB. Application of a nurse-managed inpatient smoking cessation program. Nicotine \& Tobacco Research 2002;4(2):211-22. [MEDLINE: 165]

\section{Sneed 1997 \{published data only\}}

Sneed NV, Finch NJ, Michel Y. The effect of psychosocial nursing intervention on the mood state of patients with implantable cardioverter defibrillators and their caregivers. Progress in Cardiovascular Nursing 1997;12(2):4-14. [MEDLINE: 6420]

Soskolne 1993 \{published data only\}

Soskolne V, Auslander GK. Follow-up evaluation of discharge planning by social workers in an acute-care medical center in 
Israel. Social Work in Health Care 1993;18(2):23-48. [MEDLINE: 8353]

\section{Sparacino 1997 \{published data only\}}

Sparacino PA. A nurse, algorithms, and the telephone: key resources for patients after hospital discharge. Clinical Nurse Specialist 1997;11(2):51. [MEDLINE: 15285]

\section{Stanislaw 1994 \{published data only\}}

Stanislaw AE, Wewers ME. A smoking cessation intervention with hospitalized surgical cancer patients: a pilot study. Cancer Nursing 1994;17(2):81-6. [MEDLINE: 7907]

\section{Steel 2003 \{published data only\}}

Steel O'Connor KO, Mowat DL, Scott HM, Carr PA, Dorland JL, Young Tai KF. A randomized trial of two public health nurse follow-up programs after early obstetrical discharge: an examination of breastfeeding rates, maternal confidence and utilization and costs of health services. Canadian Journal of Public Health Revue Canadienne de Sante Publique 2003;94(2):98-103. [MEDLINE: 1156]

\section{Stevens 1993 \{published data only\}}

Stevens VJ, Glasgow RE, Hollis JF, Lichtenstein E, Vogt TM. A smoking-cessation intervention for hospital patients. Medical Care 1993;31(1):65-72. [MEDLINE: 8350]

\section{Stevens 2000 \{published data only\}}

Stevens VJ, Glasgow RE, Hollis JF, Mount K. Implementation and effectiveness of a brief smoking-cessation intervention for hospital patients. Medical Care 2000;38(5):451-9. [MEDLINE: 4133]

\section{Stewart 1998 \{published data only\}}

Stewart S, Pearson S, Luke CG, Horowitz JD. Effects of homebased intervention on unplanned readmissions and out-ofhospital deaths. Journal of the American Geriatrics Society 1998;46(2):174-80. [MEDLINE: 13559]

\section{Strecher 1983 \{published data only\}}

Strecher VJ. A minimal-contact smoking cessation program in a health care setting. Public Health Reports 1983;98(5):497-502. [MEDLINE: 9973]

\section{Strinko 2000 \{published data only\}}

Strinko JM, Howard CA, Schaeffer SL, Laughlin JA, Berry MA, Turner SN. Reducing risk with telephone follow-up of patients who leave against medical advice or fail to complete an ED visit. Journal of Emergency Nursing 2000;26(3):223-32. [MEDLINE: 4068]

\section{Svahn 2002 \{published data only\}}

Svahn B-M, Remberger M, Myrback K-E, Holmberg K, Eriksson B, Hentschke $P$, et al. Home care during the pancytopenic phase after allogeneic hematopoietic stem cell transplantation is advantageous compared with hospital care. Blood 2002;100(13):4317-24. [MEDLINE: 88]

\section{Taylor 1990 \{published data only\}}

Taylor CB, Houston-Miller N, Killen JD, DeBusk RF. Smoking cessation after acute myocardial infarction: effects of a nurse-managed intervention. Annals of Internal Medicine 1990;113(2):118-23. [MEDLINE: 9051]

Taylor 1996 \{published data only\}

Taylor CB, Miller NH, Herman S, Smith PM, Sobel D, Fisher L, DeBusk RF. A nurse-managed smoking cessation program for hospitalized smokers. American Journal of Public Health 1996;86(11):1557-60. [MEDLINE: 736]

Taylor 1997 \{published data only\}

Taylor CB, Miller NH, Smith PM, DeBusk RF. The effect of a home-based, case-managed, multifactorial risk-reduction program on reducing psychological distress in patients with cardiovascular disease. Journal of Cardiopulmonary Rehabilitation 1997;17(3):157-62. [MEDLINE: 713]

Taylor-Davis 2000 \{published data only\}

Taylor-Davis S, Smiciklas-Wright H, Warland R, Achterberg C, Jensen GL, Sayer A, Shannon B. Responses of older adults to theory-based nutrition newsletters. Journal of the American Dietetic Association 2000;100(6):656-64. [MEDLINE: 4026]

\section{Thewissen 2000 \{published data only\}}

Thewissen M, Verhoeven M. Meer bereiken door betere bereikbaarheid: telefonisch spreekuur biedt onverwachte voordelen. Oncologica 2000;17(1):14-5. [MEDLINE: 15219]

\section{Thompson 1999 \{published data only\}}

Thompson DM, Kozak SE, Sheps S. Insulin adjustment by a diabetes nurse educator improves glucose control in insulinrequiring diabetic patients: a randomized trial. Canadian Medical Association Journal 1999;161(8):959-62. [MEDLINE: 4549]

\section{Tiippana 2003 \{published data only\}}

Tiippana E, Nilsson E, Kalso E. Post-thoracotomy pain after thoracic epidural analgesia: a prospective follow-up study. Acta Anaesthesiologica Scandinavica 2003;47(4):433-8. [MEDLINE: 1138]

\section{Tkachuk 2003 \{published data only\}}

Tkachuk GA, Graff LA, Martin GL, Bernstein CN. Randomized controlled trial of cognitive-behavioral group therapy for irritable bowel syndrome in a medical setting. Journal of Clinical Psychology in Medical Settings 2003;10(1):57-69. [MEDLINE: 5]

Townsend 1996 \{published data only\}

Townsend J, Frank A, Piper M. Continuing rise in emergency admissions. Visiting elderly patients at home immediately after discharge reduces emergency readmissions. BMJ 1996;313(7052):302. [MEDLINE: 6821]

\section{Turner 1996 \{published data only\}}

Turner D. Can telephone follow-up improve post-discharge outcomes?. British Journal of Nursing 1996;5(22):1361-5. [MEDLINE: 10700]

\section{Tyc 2003 \{published data only\}}

Tyc VL, Rai SN, Lensing S, Klosky JL, Stewart DB, Gattuso J. Intervention to reduce intentions to use tobacco among

Telephone follow-up, initiated by a hospital-based health professional, for postdischarge problems in patients discharged from hospital 
pediatric cancer survivors. Journal of Clinical Oncology 2003;21(7):1366-72. [MEDLINE: 1173]

Valanis 2001 \{published data only\}

Valanis B, Tanner C, Moscato S. Telephone nursing advice: the buck stops here... 34th Annual Communicating Nursing Research Conference/15th Annual WIN Assembly, "Health Care Challenges Beyond 2001: Mapping the Journey for Research and Practice," held April 19-21, 2001 in Seattle, Washington. Communicating Nursing Research 2001;34(9):224. [MEDLINE: 10775]

\section{Valanis 2002 \{published data only\}}

Valanis B, Tanner C, Moscato S, David M, Shapiro S, Izumi S. Telephone advice nursing: facilitating access to health care... 35th Annual Communicating Nursing Research Conference/16th Annual WIN Assembly, "Health Disparities: Meeting the Challenge," held April 18-20, 2002, Palm Springs, California. Communicating Nursing Research 2002;35(10):339. [MEDLINE: 11078]

\section{Valanis 2003 \{published data only\}}

Valanis B, Moscato S, Tanner C, Shapiro S, Izumi S, David M, Mayo A. Making it work: organization and processes of telephone nursing advice services. Journal of Nursing Administration 2003;33(4):216-23. [MEDLINE: 1142]

\section{Vale 2002 \{published data only\}}

Vale MJ, Jelinek MV, Best JD, Santamaria JD. Coaching patients with coronary heart disease to achieve the target cholesterol: a method to bridge the gap between evidence-based medicine and the "real world"--randomized controlled trial. Journal of Clinical Epidemiology 2002;55(3):245-52. [MEDLINE: 2457]

van Beelen 1996 \{published data only\}

van Beelen A. 'Belrondes' horen bij de service: nog even een telefoontje. Verpleegkunde Nieuws 1996;10(2):11. [MEDLINE: 15234]

\section{van Elderen 1994 \{published data only\}}

van Elderen T, Maes S, Vandenbroek Y. Effects of a healtheducation program with telephone follow-up during cardiac rehabilitation. British Journal of Clinical Psychology 1994;33:367-78. [MEDLINE: 14996]

van Elderen 2001 \{published data only\}

van Elderen T, Dusseldorp E. Lifestyle effects of group health education for patients with coronary heart disease. Psychology \& Health 2001;16(3):327-41. [MEDLINE: 14287]

\section{Varma 1999 \{published data only\}}

Varma S, McElnay JC, Hughes CM, Passmore AP, Varma M. Pharmaceutical care of patients with congestive heart failure: interventions and outcomes. Pharmacotherapy 1999;19(7):860-9. [MEDLINE: 4794]

\section{Vogel 1996 \{published data only\}}

Vogel LL. Development of community follow-up in a comprehensive rehabilitation center. Journal of Rehabilitation 1996;62(2):62-6. [MEDLINE: 10344]

\section{Vrehen 2000 \{published data only\}}

Vrehen H, Drege E, Schipper E, Knape J. Telefonische nazorg na dagbehandeling: Goede ervaringen in Utrecht. Medisch Contact 2000;55(9):312-3. [MEDLINE: 15257]

\section{Wade 1998 \{published data only\}}

Wade DT, King NS, Wenden FJ, Crawford S, Caldwell FE. Routine follow up after head injury: a second randomised controlled trial. Journal of Neurology, Neurosurgery and Psychiatry 1998;65(2):177-83. [MEDLINE: 5543]

\section{Walker 1999 \{published data only\}}

Walker BL, Nail LM, Croyle RT. Does emotional expression make a difference in reactions to breast cancer?. Oncology Nursing Forum 1999;26(6):1025-32. [MEDLINE: 4791]

Warden 2000 \{published data only\}

Warden DL, Salazar AM, Martin EM, Schwab KA, Coyle M, Walter J. A home program of rehabilitation for moderately severe traumatic brain injury patients. The DVHIP Study Group. Journal of Head Trauma Rehabilitation 2000;15(5):1092-102. [MEDLINE: 3860]

Wasson 1992 \{published data only\}

Wasson J, Gaudette C, Whaley F, Sauvigne A, Baribeau P, Welch HG. Telephone care as a substitute for routine clinic follow-up. JAMA 1992;267(13):1788-93. [MEDLINE: 933]

Weinberger 1991 \{published data only\}

Weinberger M, Tierney WM, Booher P, Katz BP. The impact of increased contact on psychosocial outcomes in patients with osteoarthritis: a randomized, controlled trial. Journal of Rheumatology 1991;18(6):849-54. [MEDLINE: 8835]

Weinberger 1993 \{published data only\}

Weinberger M, Tierney WM, Cowper PA, Katz BP, Booher PA. Cost-effectiveness of increased telephone contact for patients with osteoarthritis: a randomized, controlled trial. Arthritis and Rheumatism 1993;36(2):243-6. [MEDLINE: 8324]

Weinberger 1998 \{published data only\}

Weinberger M. Telephone-based interventions in outpatient care. Annals of the Rheumatic Diseases 1998;57(4):196-7. [MEDLINE: 5536]

\section{Weinstein 1996 \{published data only\}}

Weinstein R, Tosolin F, Ghilardi L, Zanardelli E. Psychological intervention in patients with poor compliance. Journal of Clinical Periodontology 1996;23:283-8. [MEDLINE: 12878]

Welch 2000 \{published data only\}

Welch HG, Johnson DJ, Edson R. Telephone care as an adjunct to routine medical follow-up. A negative randomized trial. Effective Clinical Practice 2000;3(3):123-30. [MEDLINE: 3522]

\section{Wells 2003 \{published data only\}}

Wells N, Hepworth JT, Murphy BA, Wujcik D, Johnson R. Improving cancer pain management through patient and family education. Journal of Pain \& Symptom Management 2003;25(4):344-56. [MEDLINE: 26]

Telephone follow-up, initiated by a hospital-based health professional, for postdischarge problems in patients discharged from hospital 
Wewers 1994 \{published data only\}

Wewers ME, Bowen JM, Stanislaw AE, Desimone VB. A nursedelivered smoking cessation intervention among hospitalized postoperative patients--influence of a smoking-related diagnosis: a pilot study. Heart \& Lung: Journal of Critical Care 1994;23(2):151-6. [MEDLINE: 7921]

\section{Wilbourne 1997 \{published data only\}}

Wilbourne J, Wilson A. Telefonische consulten van patiënten met de diabetesverpleegkundige: 'verborgen' afspraken?. EADE nieuwsbrief 1997;12(1):10-3. [MEDLINE: 15232]

\section{Wong 2001a \{published data only\}}

Wong FKY, Chow S, Wan V. Nurse telephone follow-up for A\&E patients: implications for health intervention using telephone intervention. Hong Kong Nursing Journal 2001;37(4):15. [MEDLINE: 11155]

\section{Wong 2001b \{published data only\}}

Wong SPY, Kong B, Wong J. "Geriatric care at your doorway": post discharge home follow up and direct hotline service for community living elderly. Hong Kong Nursing Journal 2001;37(2):21-6. [MEDLINE: 10783]

Wulsin 2002 \{published data only\}

Wulsin L, Liu T, Storrow A, Evans S, Dewan N, Hamilton C. A randomized, controlled trial of panic disorder treatment initiation in an emergency department chest pain center. Annals of Emergency Medicine 2002;39(2):139-43. [MEDLINE: 224]

\section{York 1997 \{published data only\}}

York R, Brown LP, Samuels P, Finkler SA, Jacobsen B, Persely CA, et al. A randomized trial of early discharge and nurse specialist transitional follow-up care of high-risk childbearing women. Nursing Research 1997;46(5):254-61. [MEDLINE: 10674]

\section{Young $\mathbf{2 0 0 0}$ \{published data only\}}

Young J, O'Connell B, McGregor S. Day surgery patients' convalescence at home: does enhanced discharge education make a difference?. Nursing and Health Sciences 2000;2(1):29-39. [MEDLINE: 10806]

\section{Zahlmann 2002 \{published data only\}}

Zahlmann G, Mertz M, Fabian E, Holle R, Kaatz H, Neubauer L, et al. Perioperative cataract OP management by means of teleconsultation. Graefes Archive for Clinical and Experimental Ophthalmology 2002;240(1):17-20. [MEDLINE: 2296]

\section{Zeegers 1997 \{published data only\}}

Zeegers A, Mistiaen P. Effectivity measurement of a telephonic follow-up after hospital admission: a study into decrease of problems in ophtalmology patients after discharge from the hospital [Abstract]. Tijdschrift voor Sociale Gezondheidszorg 1997:XXXII. [MEDLINE: 12757]

\section{Zorc 2003 \{published data only\}}

Zorc JJ, Scarfone RJ, Li Y, Hong T, Harmelin M, Grunstein L, Andre JB. Scheduled follow-up after a pediatric emergency department visit for asthma: a randomized trial. Pediatrics 2003;111(3):495-502. [MEDLINE: 1238]

\section{References to studies awaiting assessment}

Wakefield \{published data only\}

Wakefield B. Evaluating telehealth home care for elderly veterans with congestive heart failure. http://clinicaltrials.gov/ show/NCT00057200. [MEDLINE: 15602]

\section{Additional references}

\section{Alderson 2002}

Alderson A, Bero L, Grimshaw J, McAuley L, Oxman A, Zwarenstein M (eds). Cochrane Effective Practice and Organisation of Care Group. The Cochrane Library 2002, issue 4.

\section{Bandura 1977}

Bandura A. Self-efficacy. The exercise of control. New York: Freeman, 1977.

\section{Beckie 1989a}

Beckie T. A supportive-educative telephone program: impact on knowledge and anxiety after coronary artery bypass graft surgery. Heart \& Lung 1989;18(1):46-55.

\section{Bours 1998}

Bours GJJ, Ketelaars CAJ, Frederiks CMA, Abu-Saad HH, Wouters EFM. The effects of aftercare on chronic patients and frail elderly patients when discharged from hospital: a systematic review. Journal of Advanced Nursing 1998;27(5):1076-86.

\section{Bowman 1994}

Bowman GS, Howden J, Allen S, Webster RA, Thompson DR. A telephone survey of medical patients 1 week after discharge from hospital. Journal of Clinical Nursing 1994;3(6):369-73.

\section{Bull 2000}

Bull MJ. Discharge planning for older people: a review of current research. British Journal of Community Nursing 2000;5(2):70-4.

\section{Clarke 2003}

Clarke M, Oxman AD, editors. Cochrane Reviewers' Handbook 4.1.6 [updated January 2003]. In: The Cochrane Library, Issue 1 2003.

\section{Cole 2001}

Cole MG. The impact of geriatric post-discharge services on mental state. Age and Ageing 2001;30(5):415-8.

\section{Cox 2003}

Cox K, Wilson E. Follow-up for people with cancer: nurse-led services and telephone interventions. Journal of Advanced Nursing 2003;43(1):51-61.

\section{Hartford 2000}

Hartford K, Wong C. What does the literature report about postdischarge telephone interventions by nurses for coronary artery bypass graft surgery patients and their partners?. Canadian Journal of Cardiovascular Nursing 2000;11(1):27-35. 


\section{Hyde 2000}

Hyde CJ, Robert IE, Sinclair AJ. The effects of supporting discharge from hospital to home in older people. Age and Ageing 2000;29(3):271-9.

\section{Johnson 2000d}

Johnson K. Use of telephone follow-up for post-cardiac surgery patients. Intensive \& Critical Care Nursing 2000;16(3):144-50.

\section{Johnson 2003}

Johnson A, Sandford J, Tyndall J. Written and verbal information versus verbal information only for patients being discharged from acute hospital settings to home. Cochrane Database of Systematic Reviews 2003, Issue 4. [DOI: 10.1002/14651858.CD003716]

\section{Keeling 1995}

Keeling AW, Dennison PD. Nurse-initiated telephone follow-up after acute myocardial infarction: a pilot study. Heart \& Lung 1995;24(1):45-9.

\section{LeClerc 2002}

LeClerc C, Wells D, Craig D, Wilson J. Falling short of the mark: tales of life after hospital discharge. Clinical Nursing Research 2002;11(3):242-63.

\section{Lindsay 2004}

Lindsay B. Randomized controlled trials of socially complex nursing interventions: creating bias and unreliability?. Journal of Advanced Nursing 2004;45(1):84-94.

\section{Mistiaen 1999a}

Mistiaen P, Duijnhouwer E, Ettema T. The construction of a research model on post discharge problems based on a review of the literature 1990-1995. Social Work in Health Care 1999;29(4):33-68.

\section{Mistiaen 1999b}

Mistiaen P, Duijnhouwer E, Prins-Hoekstra A, Ros W, Blaylock A. Predictive validity of the BRASS-index in screening patients with post-discharge problems. Journal of Advanced Nursing 1999;30(5):1050-6

\section{Moran 1999}

Moran SJ, Jarvis S, Ewings P, Parkin FA. It's good to talk, but is it effective? A comparative study of telephone support following day surgery. Clinical Effectiveness in Nursing 1999;2(4):175-84.

\section{Naylor 2002}

Naylor MD. Transitional care of older adults. Annual Review of Nursing Research 2002;20:127-47.

\section{Parker 2002}

Parker SG, Peet SM, McPherson A, Cannaby AM, Abrams K, Baker $\mathrm{R}$, et al. A systematic review of discharge arrangements for older people. Health Technology Assessment 2002;6(4):1-183.

\section{Reiley 1996}

Reiley P, lezzoni L, Philips R, Tuchin L, Calkins D. Discharge planning: comparisons of patients' and nurses' perceptions of patients following hospital discharge. Image - the Journal of Nursing Scholarship 1996;28(2):143-7.

\section{Robinson 2002}

Robinson K, Dickersin K. Development of a highly sensitive search strategy for the retrieval of reports of controlled trials using Pubmed. International Journal of Epidemiology 2002;31:150-3.

\section{Schaeffer 2001}

Schaeffer S. Follow up with patients by telephone. ED Management 2001;13(8):89-91.

\section{Shepperd 2004}

Shepperd S, Parkes J, McClaran J, Phillips C. Discharge planning from hospital to home. Cochrane Database of Systematic Reviews 2004, Issue 1. [DOI: 10.1002/14651858.CD000313.pub2]

* Indicates the major publication for the study

\section{CHARACTERISTICS OF STUDIES}

Characteristics of included studies [ordered by study ID]

\section{Al-Asseri 2001}

Methods
DETAILS OF STUDY

AIM OF INTERVENTION: whether post-discharge care counseling was a factor in improving patients' overall compliance with treatment in a hospital setting.

AIM OF STUDY: To test whether counseling of surgical and cardiac patients by a pharmacist would improve patients' drug compliance.

STUDY DESIGN: CCT.

METHODS OF RECRUITMENT OF PARTICIPANTS: unclear.

INCLUSION CRITERIA FOR PARTICIPATION IN STUDY: at least 3 medications and having a scheduled appointment at 8 weeks in hospital.

Telephone follow-up, initiated by a hospital-based health professional, for postdischarge problems in patients discharged from hospital 
EXCLUSION CRITERIA FOR PARTICIPATION IN STUDY: mental illness.

INFORMED CONSENT OBTAINED? yes.

ETHICAL APPROVAL? unclear.

FUNDING: unclear.

\section{ASSESSMENT OF STUDY QUALITY}

ALLOCATION CONCEALMENT: unclear.

EPOC- QUALITY CRITERIA 2002: C.high risk of bias.

METHOD OF GENERATING RANDOMISATION SCHEDULE: number assigned: odd number in control, even numbers in intervention.

METHOD OF CONCEALMENT OF ALLOCATION: as above, no further information given.

BLINDING:

-PARTICIPANTS: no.

-PROVIDER/S: no.

-OUTCOME ASSESSOR/S: no.

INTENTION TO TREAT ANALYSIS: not stated.

BASELINE COMPARABILITY OF INTERVENTION AND CONTROL GROUPS: not stated.

STATISTICAL METHODS AND THEIR APPROPRIATENESS: chi-square.

CONSUMER INVOLVEMENT: not stated.

\section{Participants}

DESCRIPTION : cardiac patients / surgery patients.

GEOGRAPHIC LOCATION: Saudi Arabia.

SETTING: discharged home from an acute care setting.

NUMBER OF PARTICIPANTS:

ELIGIBLE: unclear.

RANDOMISED TO INTERVENTION: 36.

RANDOMISED TO CONTROL: 36.

INCLUDED IN ANALYSIS INTERVENTION GROUP: 36.

INCLUDED IN ANALYSIS CONTROL GROUP: 36.

AGE: RANGE OR MEAN (SD): 18-70.

GENDER (\% MALE): 50.

ETHNICITY: unclear.

PRINCIPAL HEALTH PROBLEM OR DIAGNOSIS: unclear.

OTHER HEALTH PROBLEM/S: unclear.

TREATMENT RECEIVED/RECEIVING: unclear.

OTHER SOCIAL/DEMOGRAPHIC DETAILS: not given. drug use, expected therapeutic outcome, dosage and method of administration, storage conditions, duration of therapy and what to do when a dose is missed. The pharmacist gave TFU every 3 days for up to eight weeks; also patients could call pharmacist when they needed further counseling.

DETAILS OF CONTROL: usual care.

Telephone follow-up, initiated by a hospital-based health professional, for postdischarge problems in patients discharged from hospital 
Al-Asseri 2001 (Continued)

\section{CO-INTERVENTION? yes}

DETAILS OF CO-INTERVENTIONS: access to the pharmacist by phone (in intervention group only) and counseling by pharmacist on discharge day (both groups).

DELIVERY OF INTERVENTION:

Frequency: 18. First time at day 3 after discharge. Period: 8 weeks (range 2 to 8 )

PROVIDERS: pharmacist.

INTERVENTION QUALITY: unclear how much intervention was given in each patient.

FIDELITY/INTEGRITY: unclear.

Outcomes NUMBER OF OUTCOMES: 3.

OUTCOME / TOOL / TOOL VALIDATED / METHOD OF ASSESSMENT / TIME OF ASSESSMENT:

A.Psycho-social health of patients (uncertainty, anxiety, informational needs, mood, coping, quality of life, social activity, ...): satisfaction / self-developed / no / interview / 8 weeks after discharge.

B.Physical health of patients (e.g. functional status, self-care, self-efficacy, independence, ...):

C.Other consumer oriented outcomes (e.g. treatment adherence, knowledge, adverse events, ...): compliance / self-developed / no / interview / 8 weeks after discharge. Drugs-related side-effects / self-developed / no / interview / 8 weeks after discharge.

D. Health service delivery oriented outcomes (eg. hospital readmission, health services utilization, ...):

Notes $\quad$ CHANGES IN TRIAL PROTOCOL

.CONTACT WITH AUTHOR

-POWER CALCULATION?

·RECORD IF THE STUDY WAS TRANSLATED FROM A LANGUAGE OTHER THAN ENGLISH.

-RECORD IF THE STUDY WAS A DUPLICATE PUBLICATION.

\section{Risk of bias}

Bias Authors' judgement Support for judgement

Allocation concealment? Unclear risk B - Unclear

Barnason 1995

DETAILS OF STUDY
AIM OF INTERVENTION: to enhance knowledge.

AIM OF STUDY: To examine the effectiveness of three 'survival skill' based teaching strategies for cardiac surgical patient education.

STUDY DESIGN: CCT.

METHODS OF RECRUITMENT OF PARTICIPANTS: unclear.

INCLUSION CRITERIA FOR PARTICIPATION IN STUDY: admitted for elective coronary artery bypasS surgery; not further specified.

EXCLUSION CRITERIA FOR PARTICIPATION IN STUDY: not specified.

INFORMED CONSENT OBTAINED? yes. 
Barnason 1995 (Continued)

ETHICAL APPROVAL? unclear.

FUNDING: unclear.

ASSESSMENT OF STUDY QUALITY

ALLOCATION CONCEALMENT:unclear.

EPOC- QUALITY CRITERIA 2002: C. high risk of bias.

METHOD OF GENERATING RANDOMISATION SCHEDULE: not specified.

METHOD OF CONCEALMENT OF ALLOCATION: not specified.

BLINDING:

-PARTICIPANTS: no.

-PROVIDER/S: no.

-OUTCOME ASSESSOR/S: no.

INTENTION TO TREAT ANALYSIS: not stated.

BASELINE COMPARABILITY OF INTERVENTION AND CONTROL GROUPS: not stated.

STATISTICAL METHODS AND THEIR APPROPRIATENESS: anova/t-test/chi-square.

CONSUMER INVOLVEMENT: not stated.

Participants

DESCRIPTION : cardiac patients / surgery patients.

GEOGRAPHIC LOCATION: USA.

SETTING: discharged home from an acute care setting.

NUMBER OF PARTICIPANTS:

ELIGIBLE: unclear.

RANDOMISED TO INTERVENTION: 30

RANDOMISED TO CONTROL: 30 - 30.

INCLUDED IN ANALYSIS INTERVENTION GROUP: 30

INCLUDED IN ANALYSIS CONTROL GROUP: 30 - 30.

AGE: RANGE OR MEAN (SD): 63.1 (9.8).

GENDER (\% MALE): 81.

ETHNICITY: unclear.

PRINCIPAL HEALTH PROBLEM OR DIAGNOSIS: coronary artery disease.

OTHER HEALTH PROBLEM/S: unclear.

TREATMENT RECEIVED/RECEIVING: coronary bypass surgery.

OTHER SOCIAL/DEMOGRAPHIC DETAILS: 90\% married. Average hospital stay of 6.2 days.

Interventions

DETAILS OF INTERVENTION: A teaching protocol was implemented during postdischarge week two by the cardiac rehabilitation nurse via telephone. The telephone contact was a method to reinforce the teaching content which had been previously given in the hospital. There were also opportunities for the patient to clarify any additional topics with the cardiac rehabilitation nurse.

DETAILS OF CONTROL Two control groups: one received inhospital teaching only, the other inhospital teaching plus post discharge group teaching.

CO-INTERVENTION? yes.

DETAILS OF CO-INTERVENTIONS: All groups received inhospital teaching by the cardiac rehabilitation nurse on postoperative day 5 or 6 ; the content of this inpatient teaching protocol consisted on wound

Telephone follow-up, initiated by a hospital-based health professional, for postdischarge problems in patients discharged from hospital

Copyright $\odot 2010$ The Cochrane Collaboration. Published by John Wiley \& Sons, Ltd. 
Barnason 1995 (Continued)

healing, signs and symptoms of angina/MI, guidelines for activity progression, incisional care, risk factor modification, dietary modifications and method for enrolling in cardiac rehabilitation. Patients also received two teaching booklets.

DELIVERY OF INTERVENTION

Frequency: 1.

First time at day 7 to 14 after discharge.

Period:

PROVIDERS: nurse.

INTERVENTION QUALITY: unclear.

FIDELITY/INTEGRITY: unclear.

Outcomes

NUMBER OF OUTCOMES: 2.

OUTCOME / TOOL / TOOL VALIDATED / METHOD OF ASSESSMENT / TIME OF ASSESSMENT

A. Psycho-social health of patients (uncertainty, anxiety, informational needs, mood, coping, quality of life, social activity, ..): satisfaction / self-developed / partly, adopted from Gerard and Peterson's cardiac patient learning needs inventory / unclear / unclear, but in first month after discharge.

B.Physical health of patients (eg. functional status, self-care, self-efficacy, independence, ...):

C.Other consumer oriented outcomes (eg. treatment adherence, knowledge, adverse events, ...): knowledge / self-developed: heart disease management questionnaire / partly; Kuder-Richardson coefficient 0.36 / unclear / unclear, but in first month after discharge.

D. Health service delivery oriented outcomes (eg. hospital readmission, health services utilization, ...):

Notes

- CHANGES IN TRIAL PROTOCOL

.CONTACT WITH AUTHOR

-POWER CALCULATION?

·RECORD IF THE STUDY WAS TRANSLATED FROM A LANGUAGE OTHER THAN ENGLISH.

·RECORD IF THE STUDY WAS A DUPLICATE PUBLICATION.

\section{Risk of bias}

\section{Bias \\ Authors' judgement Support for judgement}

Allocation concealment? Unclear risk B - Unclear

Beckie 1989

Methods

DETAILS OF STUDY

AIM OF INTERVENTION: to reinforce cognitive and affective information that was given to patients during hospitalisation and to supplement information about specific concerns/

AIM OF STUDY: To investigate the impact of supportive-educative telephone program on the levels of knowledge and anxiety of patients undergoing coronary artery bypass graft surgery during the first 6 weeks after hospital discharge.

STUDY DESIGN: RCT.

METHODS OF RECRUITMENT OF PARTICIPANTS: the first 74 patients scheduled.

Telephone follow-up, initiated by a hospital-based health professional, for postdischarge problems in patients discharged from hospital 
Beckie 1989 (Continued)

INCLUSION CRITERIA FOR PARTICIPATION IN STUDY: scheduled for first-time non emergency CABG/ oriented/able to read, write and speak english/ access to telephone/ no major cardiac complications/ intent to return to cardiac surgeon within 6 weeks.

EXCLUSION CRITERIA FOR PARTICIPATION IN STUDY: pyschiatric problems or history.

INFORMED CONSENT OBTAINED? yes.

ETHICAL APPROVAL? unclear.

FUNDING: unclear.

ASSESSMENT OF STUDY QUALITY

ALLOCATION CONCEALMENT:unclear.

EPOC- QUALITY CRITERIA 2002: C.high risk of bias.

METHOD OF GENERATING RANDOMISATION SCHEDULE: not specified.

METHOD OF CONCEALMENT OF ALLOCATION: not specified.

BLINDING:

-PARTICIPANTS: no.

-PROVIDER/S: no.

-OUTCOME ASSESSOR/S: yes.

INTENTION TO TREAT ANALYSIS: not stated.

BASELINE COMPARABILITY OF INTERVENTION AND CONTROL GROUPS: yes.

STATISTICAL METHODS AND THEIR APPROPRIATENESS: t-test.

CONSUMER INVOLVEMENT: not stated.

Participants

DESCRIPTION :cardiac patients / surgery patients.

GEOGRAPHIC LOCATION: Canada.

SETTING:discharged home from an acute care setting.

NUMBER OF PARTTICIPANTS:

ELIGIBLE: 87.

RANDOMISED TO INTERVENTION: 37.

RANDOMISED TO CONTROL: 37.

INCLUDED IN ANALYSIS INTERVENTION GROUP: 37.

INCLUDED IN ANALYSIS CONTROL GROUP: 37.

AGE: RANGE OR MEAN (SD): 50 to 70

GENDER (\% MALE): 86

ETHNICITY: white.

PRINCIPAL HEALTH PROBLEM OR DIAGNOSIS: coronary artery disease.

OTHER HEALTH PROBLEM/S: none.

TREATMENT RECEIVED/RECEIVING: coronary bypass surgery.

OTHER SOCIAL/DEMOGRAPHIC DETAILS: $81 \%$ married/ 58\% employed/ $57 \%$ rural homes/ $80 \%$ more than 10 years education

Interventions

DETAILS OF INTERVENTION: The supportive-educative telephone program was an interactive program involving information exchange between patient and the cardiac rehabilitation NURSE specialist through a series of 4 to 6 telephone calls initiated by the nurse during the first 6 weeks home conva-

Telephone follow-up, initiated by a hospital-based health professional, for postdischarge problems in patients discharged from hospital 37 to home (Review)

Copyright $\odot 2010$ The Cochrane Collaboration. Published by John Wiley \& Sons, Ltd. 
Beckie 1989 (Continued)

lescent period. The program was designed to assist patients to gain knowledge and improve decision making and coping skills, thereby decreasing their anxiety. A first call was made early in the first week after discharge in which the time and number of subsequent calls was made.

DETAILS OF CONTROL usual care: group and individual teaching during hospital stay, visit of self help group member, information about cardiac rehabilitation program.

CO-INTERVENTION? yes.

DETAILS OF CO-INTERVENTIONS: usual care.

\section{DELIVERY OF INTERVENTION}

Frequency: 4-6.

First time at day 3 after discharge.

Period: 6 weeks.

PROVIDERS: nurse.

INTERVENTION QUALITY: good.

FIDELITY/INTEGRITY: yes.

Outcomes

NUMBER OF OUTCOMES: 4

OUTCOME / TOOL / TOOL VALIDATED / METHOD OF ASSESSMENT / TIME OF ASSESSMENT

A.Psycho-social health of patients (uncertainty, anxiety, informational needs, mood, coping, quality of life, social activity, ..): anxiety / state-anxiety inventory (Spielberger 1983) / yes / interview / 6 weeks after discharge.

B.Physical health of patients (eg. functional status, self-care, self-efficacy, independence, ...):

C.Other consumer oriented outcomes (eg. treatment adherence, knowledge, adverse events, ..): knowledge / self-developed / partly, based on instrument of Horn and Swain (1977) / unclear / 6 weeks after discharge.

D. Health service delivery oriented outcomes (eg. hospital readmission, health services utilization, ...): readmission / hospital record / unclear / status analysis / 6 weeks after discharge calls to hospital / hospital record / unclear / status analysis / 6 weeks after discharge.

Notes - CHANGES IN TRIAL PROTOCOL

-CONTACT WITH AUTHOR

-POWER CALCULATION? yes.

-RECORD IF THE STUDY WAS TRANSLATED FROM A LANGUAGE OTHER THAN ENGLISH.

-RECORD IF THE STUDY WAS A DUPLICATE PUBLICATION.

\section{Risk of bias}

\begin{tabular}{lll}
\hline Bias & Authors' judgement & Support for judgement \\
\hline Allocation concealment? & Unclear risk & B - Unclear \\
\hline
\end{tabular}

Beney 2002

Methods DETAILS OF STUDY

AIM OF INTERVENTION: To improve patients' symptoms and/or side effects, such as delayed nausea and vomiting, as well as to detect and correct new symptoms that develop after discharge.

Telephone follow-up, initiated by a hospital-based health professional, for postdischarge problems in patients discharged from hospital 
Beney 2002 (Continued)

AIM OF STUDY: To evaluate the effect of telephone follow-up on the physical well-being dimension of health-related quality of life in patients with cancer.

STUDY DESIGN: RCT.

METHODS OF RECRUITMENT OF PARTICIPANTS: all patients admitted within a 1 year period; informed consent obtained during hospital stay.

INCLUSION CRITERIA FOR PARTICIPATION IN STUDY: patients with hematologic or solid tumor malignancies/ chemotherapy/speak English/ access to telephone.

EXCLUSION CRITERIA FOR PARTICIPATION IN STUDY: speech or hearing impairment/ mental or cognitive disorder/ live outside USA/ receiving weekly chemotherapy/ having allogeneic bone marrow transplant.

INFORMED CONSENT OBTAINED? yes.

ETHICAL APPROVAL? yes.

FUNDING: unclear.

ASSESSMENT OF STUDY QUALITY

ALLOCATION CONCEALMENT:adequate

EPOC- QUALITY CRITERIA 2002: B.moderate risk of bias.

METHOD OF GENERATING RANDOMISATION SCHEDULE: spreadsheet with a block size of 4.

METHOD OF CONCEALMENT OF ALLOCATION: each patient received a number and study assignment from the investigational pharmacist

BLINDING:

-PARTICIPANTS: no.

-PROVIDER/S: no.

-OUTCOME ASSESSOR/S: yes.

INTENTION TO TREAT ANALYSIS: yes.

BASELINE COMPARABILITY OF INTERVENTION AND CONTROL GROUPS: yes.

STATISTICAL METHODS AND THEIR APPROPRIATENESS: anova/t-test/chi-square/wilcoxon.

CONSUMER INVOLVEMENT: not stated.

Participants

DESCRIPTION: oncology patients.

GEOGRAPHIC LOCATION: USA.

SETTING: discharged home from an acute care setting.

NUMBER OF PARTICIPANTS:

ELIGIBLE: 161.

RANDOMISED TO INTERVENTION: 76

RANDOMISED TO CONTROL: 57.

INCLUDED IN ANALYSIS INTERVENTION GROUP: 66.

INCLUDED IN ANALYSIS CONTROL GROUP: 57

AGE: RANGE OR MEAN (SD): 53 (14).

GENDER (\% MALE): 73

ETHNICITY: 87\% Caucasian,

PRINCIPAL HEALTH PROBLEM OR DIAGNOSIS: hematologic or solid tumor malignancy,

OTHER HEALTH PROBLEM/S: none.

Telephone follow-up, initiated by a hospital-based health professional, for postdischarge problems in patients discharged from hospital

Copyright $\odot 2010$ The Cochrane Collaboration. Published by John Wiley \& Sons, Ltd. 
OTHER SOCIAL/DEMOGRAPHIC DETAILS: 44\% inpatient/mean Karnofsky score 83

Interventions

DETAILS OF INTERVENTION: TFU 48-72 hours after discharge by PHARMACIST. During the call patients were asked if they had experienced any problems since discharge. Information was solicited on both drug-related and non-drug related problems. When appropriate, patients were given advice, support and reinforcement of education provided the time of discharge, and appropriate follow-up was recommended.

DETAILS OF CONTROL: usual care.

\section{CO-INTERVENTION? no.}

DETAILS OF CO-INTERVENTIONS: usual care.

\section{DELIVERY OF INTERVENTION}

Frequency: 1.

First time at day 2-3 after discharge.

Period:

PROVIDERS: pharmacist.

INTERVENTION QUALITY: call-back duration was 7.4 minutes (range 0-30).

FIDELITY/INTEGRITY: intervention was given in $80 \%$ by pharmacist as intended; in $20 \%$ by student.

Outcomes NUMBER OF OUTCOMES: 5

OUTCOME / TOOL / TOOL VALIDATED / METHOD OF ASSESSMENT / TIME OF ASSESSMENT

A.Psycho-social health of patients (uncertainty, anxiety, informational needs, mood, coping, quality of life, social activity, ...): social well-being / Fact-G scale (Cella 1993) / yes / questionnaire / 3 weeks after discharge; emotional well-being / Fact-G scale (Cella 1993) / yes / questionnaire / 3 weeks after discharge.

B.Physical health of patients (eg. functional status, self-care, self-efficacy, independence, ...): functional well-being / Fact-G scale (Cella 1993) / yes / questionnaire / 3 weeks after discharge. Physical well-being / Fact-G scale (Cella 1993) / yes / questionnaire / 3 weeks after discharge.

C.Other consumer oriented outcomes (eg. treatment adherence, knowledge, adverse events, ..): symptom distress / Memorial Symptom Assessment Scale (Portenoy 1994) / yes / questionnaire / 3 weeks after discharge.

D. Health service delivery oriented outcomes (eg. hospital readmission, health services utilization, ...):

Notes $\quad$ CHANGES IN TRIAL PROTOCOL

\section{·CONTACT WITH AUTHOR}

-POWER CALCULATION? yes.

·RECORD IF THE STUDY WAS TRANSLATED FROM A LANGUAGE OTHER THAN ENGLISH.

-RECORD IF THE STUDY WAS A DUPLICATE PUBLICATION.

\section{Risk of bias}

\begin{tabular}{lll}
\hline Bias & Authors' judgement & Support for judgement \\
\hline Allocation concealment? & Low risk & A - Adequate \\
\hline
\end{tabular}


Bostrom 1996

METAILS OF STUDY
AIM OF INTERVENTION: The telephone nursing care link project was designed to provide discharged pa-
tients with a means for continuing the health education that was begun by nursing staff during their
hospitalisation.
AIM OF STUDY: To assess differences in patient satisfaction with the meeting of their healthcare educa-
tion needs among the patients who received a telephone call after discharge, those who are given the
opportunity to call and those who receive no additional telephone follow-up and to assess differences
in readmissions within 30 days of discharge between the three groups.

\section{STUDY DESIGN: CCT.}

METHODS OF RECRUITMENT OF PARTICIPANTS: Patients from 5 units were eligible during a 3 month period; using a counterbalanced method patients were allocated to one of three groups.

INCLUSION CRITERIA FOR PARTICIPATION IN STUDY: being discharged home from one of five participating units (general surgery, neurosurgery, orthopedic, general medicine, urology)/ English speaking.

EXCLUSION CRITERIA FOR PARTICIPATION IN STUDY: not specified.

INFORMED CONSENT OBTAINED? unclear.

ETHICAL APPROVAL? unclear.

FUNDING: unclear.

ASSESSMENT OF STUDY QUALITY

ALLOCATION CONCEALMENT: unclear.

EPOC- QUALITY CRITERIA 2002: C.high risk of bias.

METHOD OF GENERATING RANDOMISATION SCHEDULE: alternating scheme by time period and nursing unit.

METHOD OF CONCEALMENT OF ALLOCATION: not specified.

BLINDING:

-PARTICIPANTS: no.

-PROVIDER/S: no.

-OUTCOME ASSESSOR/S: no.

INTENTION TO TREAT ANALYSIS: not stated.

BASELINE COMPARABILITY OF INTERVENTION AND CONTROL GROUPS: no.

STATISTICAL METHODS AND THEIR APPROPRIATENESS: anova/chi-square.

CONSUMER INVOLVEMENT: not stated.

Participants

DESCRIPTION: mixed specialties.

GEOGRAPHIC LOCATION: USA.

SETTING: discharged home from an acute care setting.

NUMBER OF PARTICIPANTS:

ELIGIBLE: 1413,

RANDOMISED TO INTERVENTION: 445 ,

RANDOMISED TO CONTROL: 183 - 474,

INCLUDED IN ANALYSIS INTERVENTION GROUP: 165,

INCLUDED IN ANALYSIS CONTROL GROUP: 183 - 474,

Telephone follow-up, initiated by a hospital-based health professional, for postdischarge problems in patients discharged from hospital 
Bostrom 1996 (Continued)

\author{
AGE: RANGE OR MEAN (SD): \\ GENDER (\% MALE): \\ ETHNICITY: \\ PRINCIPAL HEALTH PROBLEM OR DIAGNOSIS: unclear. \\ OTHER HEALTH PROBLEM/S: unclear. \\ TREATMENT RECEIVED/RECEIVING: unclear. \\ OTHER SOCIAL/DEMOGRAPHIC DETAILS: not given.
}

Interventions

DETAILS OF INTERVENTION: The nurse contacted the patient 2 to 3 days after discharge. Additional calls were made as needed.

DETAILS OF CONTROL: Two control groups: one received usual care and the other were given a brochure at discharge that described the project and contained information on how to contact the nurse specialist, the hours of operation and a description of the types of questions that were appropriate for this service.

CO-INTERVENTION? no.

DETAILS OF CO-INTERVENTIONS: usual care.

DELIVERY OF INTERVENTION

Frequency: mostly 2 to 3 calls.

First time at day 1 after discharge.

Period:

PROVIDERS: nurse.

INTERVENTION QUALITY: unclear how much additional phone calls were made.

FIDELITY/INTEGRITY: unclear.

Outcomes NUMBER OF OUTCOMES: 2.

OUTCOME / TOOL / TOOL VALIDATED / METHOD OF ASSESSMENT / TIME OF ASSESSMENT

A.Psycho-social health of patients (uncertainty, anxiety, informational needs, mood, coping, quality of life, social activity, ...): satisfaction / self-developed / partly, adaptation of the Patient Learning Need Scale (Bubela, 1990) / questionnaire / 4 weeks after discharge.

B.Physical health of patients (eg. functional status, self-care, self-efficacy, independence, ...):

C.Other consumer oriented outcomes (eg. treatment adherence, knowledge, adverse events, ..):

D. Health service delivery oriented outcomes (eg. hospital readmission, health services utilization, ...): readmission / hospital record / unclear / status analysis / 4 weeks after discharge.

Notes
$\cdot$ CHANGES IN TRIAL PROTOCOL
$\cdot$ CONTACT WITH AUTHOR
$\cdot$ POWER CALCULATION?
$\cdot$ RECORD IF THE STUDY WAS TRANSLATED FROM A LANGUAGE OTHER THAN ENGLISH.
$\cdot$ RECORD IF THE STUDY WAS A DUPLICATE PUBLICATION.

\title{
Risk of bias
}

\section{Bias}

Authors' judgement Support for judgement

Telephone follow-up, initiated by a hospital-based health professional, for postdischarge problems in patients discharged from hospital 
Bostrom 1996 (Continued)
Allocation concealment?
Unclear risk
B - Unclear

Boter 2000

Methods

Methods
DETAILS OF STUDY

AIM OF INTERVENTION: to decrease postdischarge problems.

AIM OF STUDY: To investigate the effect of a nurse-initiated telephone reassurance program on postdischarge problems reported by recently discharged ophthalmic patients.

STUDY DESIGN: RCT.

METHODS OF RECRUITMENT OF PARTICIPANTS: patients were informed about the research project during their hospital stay and informed consent was obtained. Immediately after discharge patients were randomized by an independent researcher and using opaque envelopes (not published information).

INCLUSION CRITERIA FOR PARTICIPATION IN STUDY: adult ophthalmic patients/ at least 2 days in hospital/ dutch speaking.

EXCLUSION CRITERIA FOR PARTICIPATION IN STUDY: admitted from another ward or care institute to the pthalic unit/ discharged to institutional care setting/not able to answer the telephone/not having a telephone.

INFORMED CONSENT OBTAINED? yes.

ETHICAL APPROVAL? yes.

FUNDING: unclear.

ASSESSMENT OF STUDY QUALITY

ALLOCATION CONCEALMENT: adequate.

EPOC- QUALITY CRITERIA 2002: C. high risk of bias.

METHOD OF GENERATING RANDOMISATION SCHEDULE: not specified in article; additional information from authors obtained says opaque envelopes were used for randomization by an independent researcher.

METHOD OF CONCEALMENT OF ALLOCATION: randomization after discharge by independent researcher.

BLINDING:

-PARTICIPANTS: no.

-PROVIDER/S: no.

.OUTCOME ASSESSOR/S: no.

INTENTION TO TREAT ANALYSIS: not stated.

BASELINE COMPARABILITY OF INTERVENTION AND CONTROL GROUPS: yes.

STATISTICAL METHODS AND THEIR APPROPRIATENESS: t-test/u-test.

CONSUMER INVOLVEMENT: not stated.

Participants

GEOGRAPHIC LOCATION: The Netherlands.

SETTING: discharged home from an acute care setting.

Telephone follow-up, initiated by a hospital-based health professional, for postdischarge problems in patients discharged from hospital

Copyright $\odot 2010$ The Cochrane Collaboration. Published by John Wiley \& Sons, Ltd. 
Boter 2000 (Continued)

\author{
NUMBER OF PARTICIPANTS: \\ ELIGIBLE: 425. \\ RANDOMISED TO INTERVENTION: 196. \\ RANDOMISED TO CONTROL: 154. \\ INCLUDED IN ANALYSIS INTERVENTION GROUP: 143. \\ INCLUDED IN ANALYSIS CONTROL GROUP: 154.
}

AGE: RANGE OR MEAN (SD): 66.6 (16.1).

GENDER (\% MALE): 43.

ETHNICITY: unclear.

PRINCIPAL HEALTH PROBLEM OR DIAGNOSIS: ophthalmic disease (cataract 43\%, glaucoma 15\%, retina disorder $14 \%$, cornea disorder $13 \%)$.

OTHER HEALTH PROBLEM/S: 93\% self-supporting in ADL/IADL.

TREATMENT RECEIVED/RECEIVING: ophthalmic surgery.

OTHER SOCIAL/DEMOGRAPHIC DETAILS: 38\% living alone.

Interventions

DETAILS OF INTERVENTION: Patients were phoned 3-6 days after discharge by an experienced nurse. Before calling the nurse went through a structured form containing relevant information about the patient's admission and discharge conditions. During the call, the nurse used a structured interview schedule, covering 10 aspects. all aspects were discussed with the patient. Six nurses participated in the project.

DETAILS OF CONTROL: usual care.

CO-INTERVENTION? no.

DETAILS OF CO-INTERVENTIONS: usual care.

DELIVERY OF INTERVENTION

Frequency: 1.

First time at day 3-6 after discharge.

Period:

PROVIDERS: nurse.

INTERVENTION QUALITY: good.

FIDELITY/INTEGRITY: unclear.

OUTCOME / TOOL / TOOL VALIDATED / METHOD OF ASSESSMENT / TIME OF ASSESSMENT A.Psycho-social health of patients (uncertainty, anxiety, informational needs, mood, coping, quality of life, social activity, ...): Informational needs / self-developed / partly, adaptation of the Patient Learning Need Scale (Bubela, 1990) / questionnaire / 1 and 4 weeks after discharge. Uncertainty / Mishel Uncertainty in Illness Scale (1989) / yes / questionnaire / 1 and 4 weeks after discharge. Emotional functioning / Problems after Discharge Questionnaire (Mistiaen, 1997) / yes / questionnaire / 1 and 4 weeks after discharge.

B.Physical health of patients (eg. functional status, self-care, self-efficacy, independence, ..): Functional limitations / Problems after Discharge Questionnaire (Mistiaen, 1997) / yes / questionnaire / 1 and 4 weeks after discharge.

C.Other consumer oriented outcomes (eg. treatment adherence, knowledge, adverse events, ..):

D. Health service delivery oriented outcomes (eg. hospital readmission, health services utilization, ..): 
Boter 2000 (Continued)

\author{
-CONTACT WITH AUTHOR \\ -POWER CALCULATION? yes. \\ -RECORD IF THE STUDY WAS TRANSLATED FROM A LANGUAGE OTHER THAN ENGLISH. \\ ·RECORD IF THE STUDY WAS A DUPLICATE PUBLICATION.
}

\begin{tabular}{lll}
\hline Risk of bias & \\
\hline Bias & Authors' judgement & Support for judgement \\
\hline Allocation concealment? & Low risk & A - Adequate \\
\hline
\end{tabular}

\title{
Chande 1994
}

Methods

\section{DETAILS OF STUDY}

AIM OF INTERVENTION: to improve parental compliance with primary care follow-up.

AIM OF STUDY: It was hypothesized that physician initiated follow-up phone calls to parents of moderately ill children seen in the pediatric emergency department would improve parental compliance with primary care follow-up.

\section{STUDY DESIGN: CCT.}

METHODS OF RECRUITMENT OF PARTICIPANTS: unclear.

INCLUSION CRITERIA FOR PARTICIPATION IN STUDY: patients between 6 months and 8 years of age treated as outpatients in the pediatric emergency department for pneumonia or croup or asthma or bronchiolitis or vomiting or fever eci or fever $>39.5$ or seizure with fever/ having telephone,

EXCLUSION CRITERIA FOR PARTICIPATION IN STUDY: not specified,

INFORMED CONSENT OBTAINED? unclear.

ETHICAL APPROVAL? yes.

FUNDING: unclear.

ASSESSMENT OF STUDY QUALITY

ALLOCATION CONCEALMENT: unclear.

EPOC- QUALITY CRITERIA 2002: C.high risk of bias.

METHOD OF GENERATING RANDOMISATION SCHEDULE: not specified.

METHOD OF CONCEALMENT OF ALLOCATION: not specified.

BLINDING:

-PARTICIPANTS: no.

-PROVIDER/S: no.

-OUTCOME ASSESSOR/S: yes.

INTENTION TO TREAT ANALYSIS: not stated.

BASELINE COMPARABILITY OF INTERVENTION AND CONTROL GROUPS: not stated.

STATISTICAL METHODS AND THEIR APPROPRIATENESS: chi-square.

CONSUMER INVOLVEMENT: not stated. 
Chande 1994 (Continued)

Participants
DESCRIPTION : ED patients / pediatric patients.

GEOGRAPHIC LOCATION: USA.

SETTING: discharged home from an acute care setting.

NUMBER OF PARTICIPANTS:

ELIGIBLE: 305.

RANDOMISED TO INTERVENTION: 133.

RANDOMISED TO CONTROL: 132 - - .

INCLUDED IN ANALYSIS INTERVENTION GROUP: 133.

INCLUDED IN ANALYSIS CONTROL GROUP: 132 - - .

AGE: RANGE OR MEAN (SD): 3.2 (2.3).

GENDER (\% MALE): 63.

ETHNICITY: 83\% afro-american.

PRINCIPAL HEALTH PROBLEM OR DIAGNOSIS:pneumonia or croup or asthma or bronchiolitis or vomiting or fever eci or fever $>39.5$ or seizure with fever

OTHER HEALTH PROBLEM/S: unclear.

TREATMENT RECEIVED/RECEIVING: unclear.

OTHER SOCIAL/DEMOGRAPHIC DETAILS: $70.5 \%$ on medical assistance insurance; $2.3 \%$ had no primary care physician.

Interventions

DETAILS OF INTERVENTION: families in the intervention group were called by a physician within 12-30 hours after discharge. At that time they were reminded to fill their prescriptions, to call their regular doctors, and to follow-up any other specific instructions that had been documented on the discharge sheet. Parents were also given the opportunity to ask questions about other issues related to their child's health.

DETAILS OF CONTROL: usual care.

CO-INTERVENTION? no.

DETAILS OF CO-INTERVENTIONS: usual care.

DELIVERY OF INTERVENTION

Frequency: 1 .

First time at day 1-2 after discharge.

Period:

PROVIDERS: physician.

INTERVENTION QUALITY: unclear.

FIDELITY/INTEGRITY: unclear.

Outcomes
OUTCOME / TOOL / TOOL VALIDATED / METHOD OF ASSESSMENT / TIME OF ASSESSMENT

A.Psycho-social health of patients (uncertainty, anxiety, informational needs, mood, coping, quality of life, social activity, ..):

B.Physical health of patients (eg. functional status, self-care, self-efficacy, independence, ...):

C.Other consumer oriented outcomes (eg. treatment adherence, knowledge, adverse events, ..): compliance / self-developed / no / telephone interview / 10-20 days after discharge.

D. Health service delivery oriented outcomes (eg. hospital readmission, health services utilization, ..): 
Chande 1994 (Continued)

Notes
- CHANGES IN TRIAL PROTOCOL

-CONTACT WITH AUTHOR

-POWER CALCULATION?

·RECORD IF THE STUDY WAS TRANSLATED FROM A LANGUAGE OTHER THAN ENGLISH.

·RECORD IF THE STUDY WAS A DUPLICATE PUBLICATION.

\section{Risk of bias}

Bias Authors' judgement Support for judgement

Allocation concealment? Unclear risk B - Unclear

Dudas 2001

Methods
DETAILS OF STUDY

AIM OF INTERVENTION: A mechanism that may improve patient satisfaction and clinical outcomes at the time of discharge is the use of follow-up telephone calls.

AIM OF STUDY: whether pharmacist involved in discharge planning can improve patient satisfaction and outcomes by providing telephone follow-up after hospital discharge.

STUDY DESIGN: CCT.

METHODS OF RECRUITMENT OF PARTICIPANTS: all patients admitted within a 1 year period, who received a pharmacy-facilitated discharge (= provision of patient counseling on all discharge medications, assistance in obtaining medications and completing insurance forms) and are discharged home.

INCLUSION CRITERIA FOR PARTICIPATION IN STUDY: Patients from a general medical service discharged home with a pharmacy-facilitated discharge

EXCLUSION CRITERIA FOR PARTICIPATION IN STUDY: discharged to nursing home or other care facility/ homeless/ non-English speaker/unable to participate in a telephone conversation or complete a written survey

INFORMED CONSENT OBTAINED? yes

ETHICAL APPROVAL? yes

FUNDING: yes

ASSESSMENT OF STUDY QUALITY

ALLOCATION CONCEALMENT: unclear.

EPOC- QUALITY CRITERIA 2002: C.high risk of bias.

METHOD OF GENERATING RANDOMISATION SCHEDULE: not specified.

METHOD OF CONCEALMENT OF ALLOCATION: not specified.

BLINDING:

-PARTICIPANTS: no.

-PROVIDER/S: no.

-OUTCOME ASSESSOR/S: no.

INTENTION TO TREAT ANALYSIS: yes. 
Dudas 2001 (Continued)

BASELINE COMPARABILITY OF INTERVENTION AND CONTROL GROUPS: not stated.

STATISTICAL METHODS AND THEIR APPROPRIATENESS: chi-square/t-test.

CONSUMER INVOLVEMENT: not stated.

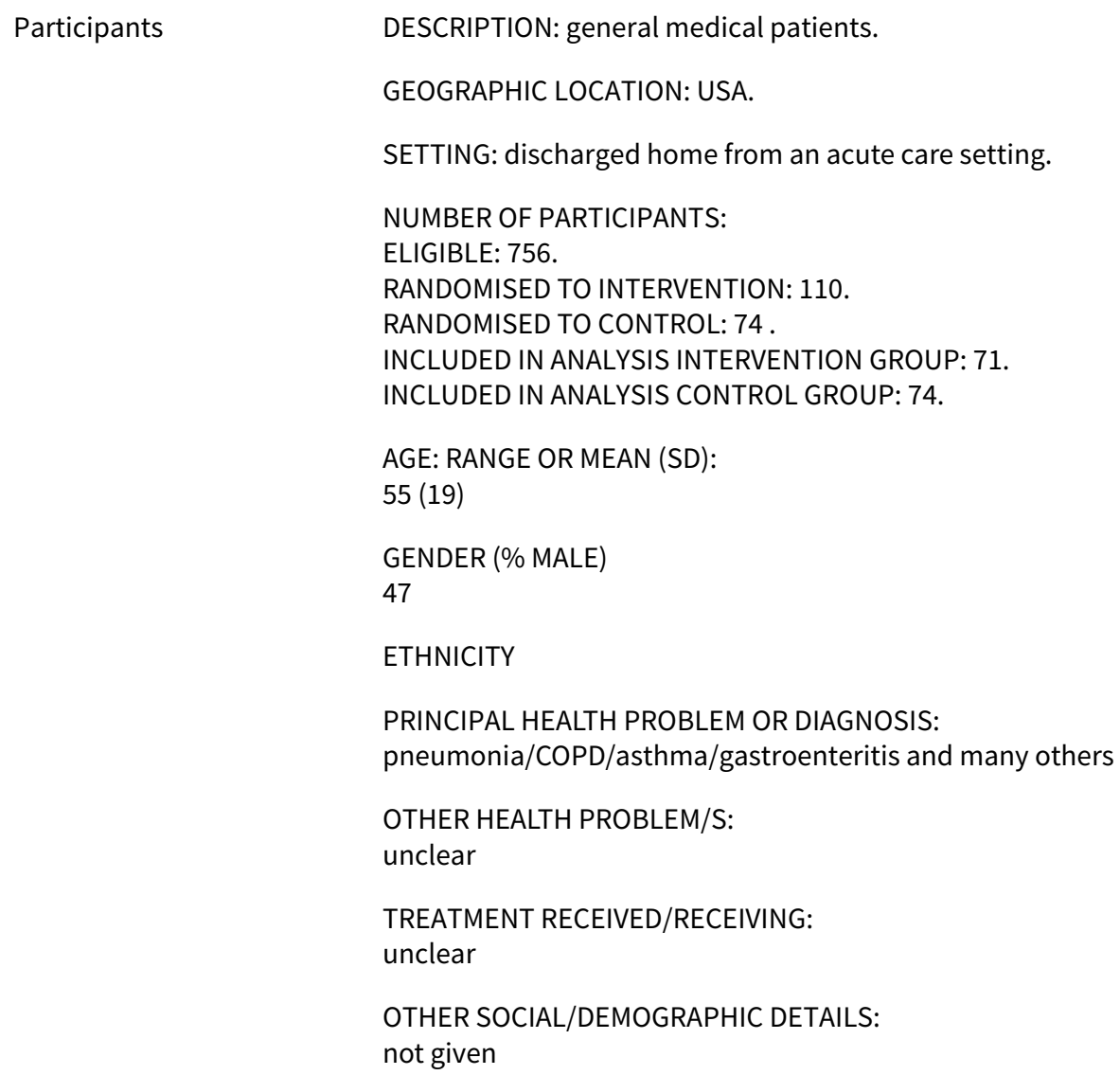

DETAILS OF INTERVENTION: within 2 days after discharge patients received a telephone call from a member of the pharmacy service. The content of the call followed a script to ensure consistency. During the call patients were asked how they had been feeling since returning home, if they had any questions regarding follow-up appointments or the in-hospital care, if they were able to obtain their medication, if they had experienced any medication related side-effects, and if they had any other question or concern.

DETAILS OF CONTROL: usual care.

CO-INTERVENTION? no.

DETAILS OF CO-INTERVENTIONS: usual care.

\section{DELIVERY OF INTERVENTION}

Frequency: 1.

First time at day 2 after discharge.

Period:

PROVIDERS: pharmacist.

INTERVENTION QUALITY: good.

FIDELITY/INTEGRITY: yes. 
B.Physical health of patients (eg. functional status, self-care, self-efficacy, independence, ...):

C.Other consumer oriented outcomes (eg. treatment adherence, knowledge, adverse events, ...):

D. Health service delivery oriented outcomes (eg. hospital readmission, health services utilization, ..):

Readmission / hospital record / unclear / status analysis / 4 weeks after discharge.

ED-visits / hospital record / unclear / status analysis / 4 weeks after discharge.

Notes

- CHANGES IN TRIAL PROTOCOL

-CONTACT WITH AUTHOR

-POWER CALCULATION?

-RECORD IF THE STUDY WAS TRANSLATED FROM A LANGUAGE OTHER THAN ENGLISH.

·RECORD IF THE STUDY WAS A DUPLICATE PUBLICATION. Yes (see reference Dudas 2001).

\section{Risk of bias}

Bias Authors' judgement Support for judgement

Allocation concealment? Unclear risk B - Unclear

Emerson 2000

Methods

DETAILS OF STUDY

AIM OF INTERVENTION: provider initiated follow-up calls to a patient may provide the opportunity to decrease the frequency of unnecessary return office visits; this could save money and time for both provider and patient.

AIM OF STUDY: to determine the effects of follow-up telephone calls on the number of return office visits of vasectomy patients.

STUDY DESIGN: CCT.

METHODS OF RECRUITMENT OF PARTICIPANTS: in the first 3 months all patients were considered intervention patients and received TFU; the next 3 months all patients were considered control patients and received only written postoperative instructions and no TFU.

INCLUSION CRITERIA FOR PARTICIPATION IN STUDY: vasectomy patients from an urology group.

EXCLUSION CRITERIA FOR PARTICIPATION IN STUDY: not specified.

INFORMED CONSENT OBTAINED? no,

ETHICAL APPROVAL? yes.

FUNDING: unclear.

ASSESSMENT OF STUDY QUALITY

ALLOCATION CONCEALMENT: inadequate.

EPOC- QUALITY CRITERIA 2002: C.high risk of bias.

METHOD OF GENERATING RANDOMISATION SCHEDULE: not specified.

Telephone follow-up, initiated by a hospital-based health professional, for postdischarge problems in patients discharged from hospital 
Emerson 2000 (Continued)

METHOD OF CONCEALMENT OF ALLOCATION: not specified.

BLINDING:

-PARTICIPANTS: no.

-PROVIDER/S: no.

-OUTCOME ASSESSOR/S: no.

INTENTION TO TREAT ANALYSIS: not stated.

BASELINE COMPARABILITY OF INTERVENTION AND CONTROL GROUPS: not stated.

STATISTICAL METHODS AND THEIR APPROPRIATENESS: none.

CONSUMER INVOLVEMENT: not stated.

\section{Participants}

DESCRIPTION: surgery patients.

GEOGRAPHIC LOCATION: USA.

SETTING: discharged home from an acute care setting.

NUMBER OF PARTICIPANTS:

ELIGIBLE: 27.

RANDOMISED TO INTERVENTION: 11.

RANDOMISED TO CONTROL: 16.

INCLUDED IN ANALYSIS INTERVENTION GROUP: 11.

INCLUDED IN ANALYSIS CONTROL GROUP: 16

AGE: RANGE OR MEAN (SD):

GENDER (\% MALE)

100

ETHNICITY

PRINCIPAL HEALTH PROBLEM OR DIAGNOSIS:

unclear

OTHER HEALTH PROBLEM/S:

unclear

TREATMENT RECEIVED/RECEIVING:

vasectomy

OTHER SOCIAL/DEMOGRAPHIC DETAILS:

not given

DETAILS OF INTERVENTION: A telephone call that is specific to the needs/concerns of a vasectomy patient made within 24 to 48 hours of the procedure by a nurse regarding pain, swelling, redness and fever.

DETAILS OF CONTROL: usual care.

CO-INTERVENTION? no.

DETAILS OF CO-INTERVENTIONS: usual care.

DELIVERY OF INTERVENTION

Frequency: 1.

First time at day 1-2 after discharge.

Period:

PROVIDERS: nurse.

INTERVENTION QUALITY: unclear.

Telephone follow-up, initiated by a hospital-based health professional, for postdischarge problems in patients discharged from hospital

Copyright $\odot 2010$ The Cochrane Collaboration. Published by John Wiley \& Sons, Ltd. 


Outcomes NUMBER OF OUTCOMES: 1

OUTCOME / TOOL / TOOL VALIDATED / METHOD OF ASSESSMENT / TIME OF ASSESSMENT

A.Psycho-social health of patients (uncertainty, anxiety, informational needs, mood, coping, quality of life, social activity, ..):

B.Physical health of patients (eg. functional status, self-care, self-efficacy, independence, ...):

C.Other consumer oriented outcomes (eg. treatment adherence, knowledge, adverse events, ...):

D. Health service delivery oriented outcomes (eg. hospital readmission, health services utilization, ..): Unnecessary return office visits / hospital record / unclear / status analysis / 4 weeks after discharge.

$\begin{array}{ll}\text { Notes } & \cdot \text { CHANGES IN TRIAL PROTOCOL } \\ & \cdot \text { CONTACT WITH AUTHOR } \\ & \cdot \text { POWER CALCULATION? } \\ & \cdot \text { RECORD IF THE STUDY WAS TRANSLATED FROM A LANGUAGE OTHER THAN ENGLISH. } \\ & \cdot \text { RECORD IF THE STUDY WAS A DUPLICATE PUBLICATION. }\end{array}$

\section{Risk of bias}

\begin{tabular}{lll}
\hline Bias & Authors' judgement & Support for judgement \\
\hline Allocation concealment? & High risk & C - Inadequate \\
\hline
\end{tabular}

Fallis 2001

Methods
DETAILS OF STUDY

AIM OF INTERVENTION: To assess the patient's level of recuperation, evaluate the care received and identify inadequacies of the process; furthermore it demonstrates a sense of caring about patients and assists in marketing an ambulatory surgery program.

AIM OF STUDY: To investigate the post-discharge follow-up required for patients who have undergone laparoscopic cholecystectomy on an outpatient basis and to determine if there was a significant difference in mean concern scores and satisfaction level of patients followed up by a home visit versus a telephone call.

\section{STUDY DESIGN: CCT.}

METHODS OF RECRUITMENT OF PARTICIPANTS: a convenience sample of patients scheduled for elective or urgent laparoscopic cholecystectomy.

INCLUSION CRITERIA FOR PARTICIPATION IN STUDY: not requiring postoperative admission; willing to be discharged on the day of operation, have a responsible caregiver and have a telephone.

EXCLUSION CRITERIA FOR PARTICIPATION IN STUDY: open cholecystectomy.

INFORMED CONSENT OBTAINED? yes.

ETHICAL APPROVAL? yes.

FUNDING: yes.

\section{ASSESSMENT OF STUDY QUALITY}


Fallis 2001 (Continued)

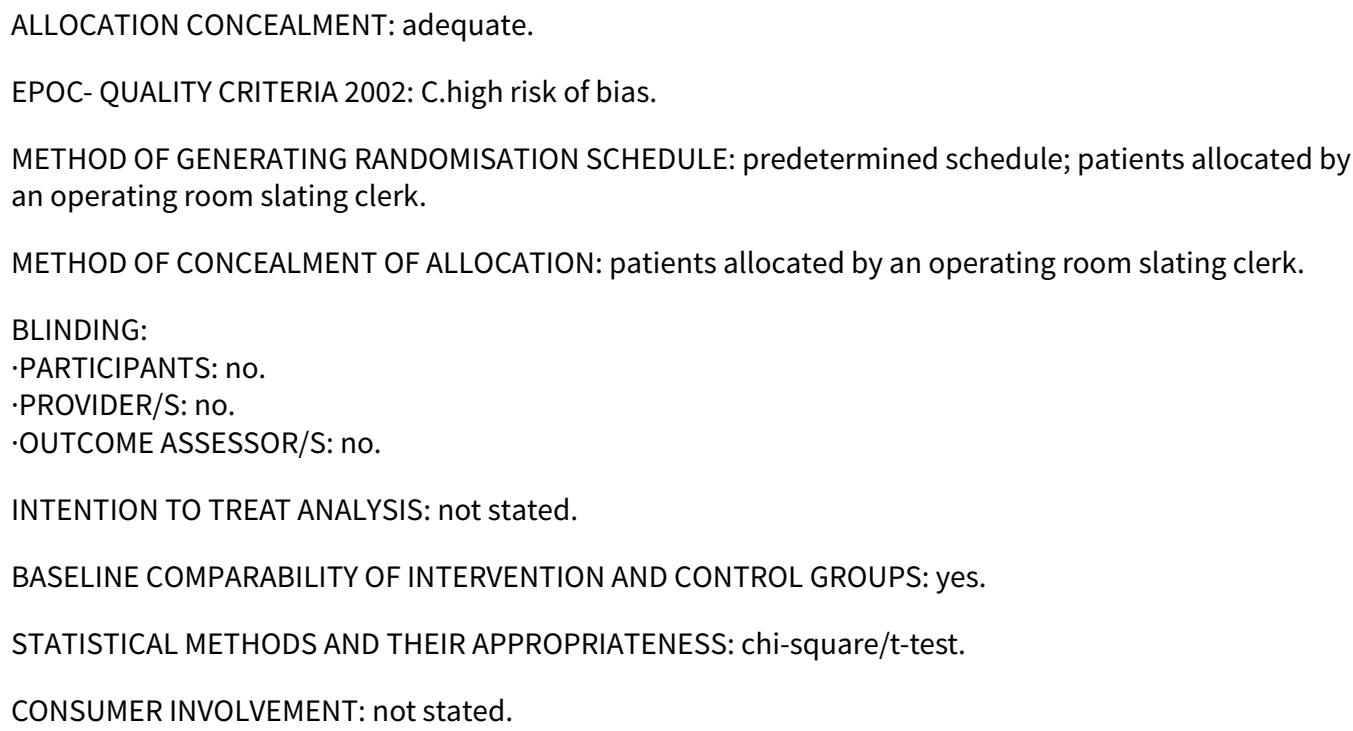

INTERVENTION QUALITY: unclear. 
Fallis 2001 (Continued)

FIDELITY/INTEGRITY: unclear.

Outcomes NUMBER OF OUTCOMES: 4

OUTCOME / TOOL / TOOL VALIDATED / METHOD OF ASSESSMENT / TIME OF ASSESSMENT

A.Psycho-social health of patients (uncertainty, anxiety, informational needs, mood, coping, quality of life, social activity, ..): Satisfaction / self-developed / no / telephone interview / 2 days after discharge patient concerns / self-developed / no / telephone interview / 2 days after discharge.

B.Physical health of patients (eg. functional status, self-care, self-efficacy, independence, ...):

C.Other consumer oriented outcomes (eg. treatment adherence, knowledge, adverse events, ...):

D. Health service delivery oriented outcomes (eg. hospital readmission, health services utilization, ..): Readmission / hospital record / unclear / status analysis / 4 weeks after discharge. ED-visits / hospital record / unclear / status analysis / 4 weeks after discharge.

Notes

-CHANGES IN TRIAL PROTOCOL

-CONTACT WITH AUTHOR

·POWER CALCULATION?

-RECORD IF THE STUDY WAS TRANSLATED FROM A LANGUAGE OTHER THAN ENGLISH.

-RECORD IF THE STUDY WAS A DUPLICATE PUBLICATION.

\section{Risk of bias}

\begin{tabular}{lll}
\hline Bias & Authors' judgement & Support for judgement \\
\hline Allocation concealment? & Low risk & A - Adequate \\
\hline
\end{tabular}

\section{Faulkner 2000}

\section{Methods}

DETAILS OF STUDY

AIM OF INTERVENTION: To enhance compliance and improve outcomes.

AIM OF STUDY: To evaluate the impact of personalized telephone follow-up on compliance rates in high-risk hypercholesteraemic patients receiving combination drug therapy.

\section{STUDY DESIGN: RCT.}

METHODS OF RECRUITMENT OF PARTICIPANTS: During a 7 month period patients who had undergone CABG surgery or percutaneous transluminal coronary angioplasty were eligible; patients were recruited from the coronary care unit.

INCLUSION CRITERIA FOR PARTICIPATION IN STUDY: CABG or PTCA patients/ baseline fasting LDL above $130 \mathrm{mg} / \mathrm{dl} /$ able to read, understand and speak English/ have telephone at home

EXCLUSION CRITERIA FOR PARTICIPATION IN STUDY: serum transaminase level twice above nor$\mathrm{mal} /$ concomitant therapy with cyclosporine, warfarin or erythromycin/history of gastrointestinal disease.

INFORMED CONSENT OBTAINED? yes.

ETHICAL APPROVAL? unclear.

FUNDING: unclear. 
Faulkner 2000 (Continued)

\author{
ASSESSMENT OF STUDY QUALITY \\ ALLOCATION CONCEALMENT: unclear. \\ EPOC- QUALITY CRITERIA 2002: C. high risk of bias. \\ METHOD OF GENERATING RANDOMISATION SCHEDULE: a computer generated list of random numbers. \\ METHOD OF CONCEALMENT OF ALLOCATION: unclear. \\ BLINDING: \\ -PARTICIPANTS: no. \\ -PROVIDER/S: no. \\ -OUTCOME ASSESSOR/S: no.
}

INTENTION TO TREAT ANALYSIS: not stated.

BASELINE COMPARABILITY OF INTERVENTION AND CONTROL GROUPS: yes.

STATISTICAL METHODS AND THEIR APPROPRIATENESS: chi-square/t-test.

CONSUMER INVOLVEMENT: not stated.

Participants

DESCRIPTION : surgery patients / cardiac patients.

GEOGRAPHIC LOCATION: USA.

SETTING: discharged home from an acute care setting.

NUMBER OF PARTICIPANTS:

ELIGIBLE: 30.

RANDOMISED TO INTERVENTION: 15.

RANDOMISED TO CONTROL: 15.

INCLUDED IN ANALYSIS INTERVENTION GROUP: 15.

INCLUDED IN ANALYSIS CONTROL GROUP: 15.

AGE: RANGE OR MEAN (SD): 62.5 (12).

GENDER (\% MALE): 57.

ETHNICITY: 70\% Caucasian.

PRINCIPAL HEALTH PROBLEM OR DIAGNOSIS: cardiovascular disease.

OTHER HEALTH PROBLEM/S: hypercholesterolaemia.

TREATMENT RECEIVED/RECEIVING: CABG (20x)/PTCA(10).

OTHER SOCIAL/DEMOGRAPHIC DETAILS:

Interventions

DETAILS OF INTERVENTION: A pharmacist telephoned patients at their home every week for 12 weeks. To ensure consistency, the same pharmacist was involved in each patient contact and a standard set of questions was asked. Emphasis was placed on the importance of therapy in reducing the risk of recurrent cardiac events. Patients were questioned about when and where prescriptions were filled, how they paid their prescriptions, potential side-effects, overall well-being, and specific reasons for noncompliance when applicable.

DETAILS OF CONTROL: usual care.

CO-INTERVENTION? yes.

DETAILS OF CO-INTERVENTIONS: in hospital all patients were extensively counseled on the appropriate use of the drugs and all patients received dietary instructions.

DELIVERY OF INTERVENTION

Telephone follow-up, initiated by a hospital-based health professional, for postdischarge problems in patients discharged from hospital 54 to home (Review)

Copyright $\odot 2010$ The Cochrane Collaboration. Published by John Wiley \& Sons, Ltd. 
Faulkner 2000 (Continued)

Frequency: 12.

First time at day 7 after discharge.

Period: 12 weeks.

PROVIDERS: pharmacist.

INTERVENTION QUALITY: unclear.

FIDELITY/INTEGRITY: unclear.

Outcomes NUMBER OF OUTCOMES: 2

OUTCOME / TOOL / TOOL VALIDATED / METHOD OF ASSESSMENT / TIME OF ASSESSMENT

A.Psycho-social health of patients (uncertainty, anxiety, informational needs, mood, coping, quality of life, social activity, ..):

B.Physical health of patients (eg. functional status, self-care, self-efficacy, independence, ...):

C.Other consumer oriented outcomes (e.g. treatment adherence, knowledge, adverse events, ..): Compliance / pill count / no / pill count at the clinic visit / 6 and 12 weeks after discharge and at 1 and 2 years. Lipid-profiles / blood sample / unclear / blood analysis / 6 and 12 weeks after discharge.

D. Health service delivery oriented outcomes (e.g. hospital readmission, health services utilization, ..):

Notes $\quad$ CHANGES IN TRIAL PROTOCOL

NB: for the review only outcomes at 6 and 12 weeks were analyzed

·CONTACT WITH AUTHOR

-POWER CALCULATION? yes.

·RECORD IF THE STUDY WAS TRANSLATED FROM A LANGUAGE OTHER THAN English.

-RECORD IF THE STUDY WAS A DUPLICATE PUBLICATION.

\section{Risk of bias}

\begin{tabular}{lll}
\hline Bias & Authors' judgement & Support for judgement \\
\hline Allocation concealment? & Unclear risk & B - Unclear \\
\hline
\end{tabular}

\section{Garding 1988}

\section{Methods}

DETAILS OF STUDY

AIM OF INTERVENTION: to increase patient knowledge in six content areas.

AIM OF STUDY: to investigate the effect of a planned telephone follow-up program to provide information and support to post myocardial infarction patients at home in the 6 to 8 week period after hospital discharge.

\section{STUDY DESIGN: CCT.}

METHODS OF RECRUITMENT OF PARTICIPANTS: all patients entering the coronary care unit with a diagnosis of MI were eligible; further procedure unclear.

INCLUSION CRITERIA FOR PARTICIPATION IN STUDY: Myocardial infarction/ able to communicate in english/ have a telephone.

EXCLUSION CRITERIA FOR PARTICIPATION IN STUDY: disoriented to time place or person/ history of previous $\mathrm{MI} /$ psychiatric history/ too ill/ not able to return at the clinic at 2 months afterwards.

Telephone follow-up, initiated by a hospital-based health professional, for postdischarge problems in patients discharged from hospital 
INFORMED CONSENT OBTAINED? unclear.

ETHICAL APPROVAL? unclear.

FUNDING: unclear.

ASSESSMENT OF STUDY QUALITY

ALLOCATION CONCEALMENT: unclear.

EPOC- QUALITY CRITERIA 2002: B.moderate risk of bias.

METHOD OF GENERATING RANDOMISATION SCHEDULE: not specified.

METHOD OF CONCEALMENT OF ALLOCATION: not specified.

BLINDING:

-PARTICIPANTS: no.

-PROVIDER/S: no.

-OUTCOME ASSESSOR/S: yes.

INTENTION TO TREAT ANALYSIS: not stated.

BASELINE COMPARABILITY OF INTERVENTION AND CONTROL GROUPS: yes.

STATISTICAL METHODS AND THEIR APPROPRIATENESS: t-test.

CONSUMER INVOLVEMENT: not stated.

Participants

DESCRIPTION: cardiac patients.

GEOGRAPHIC LOCATION: Canada.

SETTING: discharged home from an acute care setting.

NUMBER OF PARTTICIPANTS:

ELIGIBLE: 59.

RANDOMISED TO INTERVENTION: 29.

RANDOMISED TO CONTROL: 30.

INCLUDED IN ANALYSIS INTERVENTION GROUP: 25.

INCLUDED IN ANALYSIS CONTROL GROUP: 26.

AGE: RANGE OR MEAN (SD): 54.

GENDER (\% MALE): 86

ETHNICITY: not clear.

PRINCIPAL HEALTH PROBLEM OR DIAGNOSIS: myocardial infarction.

OTHER HEALTH PROBLEM/S: unclear.

TREATMENT RECEIVED/RECEIVING: unclear.

OTHER SOCIAL/DEMOGRAPHIC DETAILS:

Interventions

DETAILS OF INTERVENTION: the cardiac rehabilitation research nurse made the follow-up phone calls; they assessed understanding of teaching done before discharge. Information that was unclear or confusing was clarified and new information introduced. To promote retention of information, topic areas addressed during each call included each of the six teaching areas of the study. Time was dependent on the patient's difficulty or ease in remembering or understanding the information provided. Approximately 3 calls were made to each subject; additional follow-up was based on the nurse assessment of the subject's knowledge.

DETAILS OF CONTROL: usual care.

Telephone follow-up, initiated by a hospital-based health professional, for postdischarge problems in patients discharged from hospital

Copyright $\odot 2010$ The Cochrane Collaboration. Published by John Wiley \& Sons, Ltd. 
Garding 1988 (Continued)

\section{CO-INTERVENTION? yes.}

DETAILS OF CO-INTERVENTIONS: inhospital teaching.

\section{DELIVERY OF INTERVENTION}

Frequency: 3.

First time at day unclear after discharge.

Period: 6-8 weeks.

PROVIDERS: nurse.

INTERVENTION QUALITY: unclear.

FIDELITY/INTEGRITY: unclear.

\section{Outcomes}

\section{NUMBER OF OUTCOMES: 1}

OUTCOME / TOOL / TOOL VALIDATED / METHOD OF ASSESSMENT / TIME OF ASSESSMENT

A.Psycho-social health of patients (uncertainty, anxiety, informational needs, mood, coping, quality of life, social activity, ..):

B.Physical health of patients (eg. functional status, self-care, self-efficacy, independence, ...):

C.Other consumer oriented outcomes (eg. treatment adherence, knowledge, adverse events, ...): Knowledge / self-developed / partly, based on instrument of Horn and Swain (1977) and interrater reliability testing / telephone interview / unclear, probably at 8 weeks post discharge.

D. Health service delivery oriented outcomes (e.g. hospital readmission, health services utilization, ..):

Notes

- CHANGES IN TRIAL PROTOCOL

-CONTACT WITH AUTHOR

-POWER CALCULATION?

·RECORD IF THE STUDY WAS TRANSLATED FROM A LANGUAGE OTHER THAN ENGLISH.

-RECORD IF THE STUDY WAS A DUPLICATE PUBLICATION.

\section{Risk of bias}

\section{Bias Authors' judgement Support for judgement}

Allocation concealment? Unclear risk B - Unclear

Gombeski 1993

DETAILS OF STUDY
AIM OF INTERVENTION: to resolve past and current problems, to provide more personalized care, to
increase patient satisfaction and thereby increasing the likelihood they will return and recommend
the institution to family and friends and so establishing a competitive advantage over local health
providers who do not have such a program.

AIM OF STUDY: it was hypothesized that calling discharged patients 3 weeks after leaving the hospital would provide an opportunity to correct any problems, offer additional service, and reinforce the hospital's concern for the patient's medical recovery.

STUDY DESIGN: CCT. 
Gombeski 1993 (Continued)

METHODS OF RECRUITMENT OF PARTICIPANTS: all patients discharged from a surgical unit were identified through the hospital's database shortly after they left the hospital.

INCLUSION CRITERIA FOR PARTICIPATION IN STUDY: discharged from surgery department (general surgery or otorhinolaryngology)/ had at least one overnight stay in the hospital/not being readmitted before scheduled telephone follow-up.

EXCLUSION CRITERIA FOR PARTICIPATION IN STUDY: international patients/ being discharged to a nursing home.

INFORMED CONSENT OBTAINED? no.

ETHICAL APPROVAL? unclear.

FUNDING: unclear.

ASSESSMENT OF STUDY QUALITY

ALLOCATION CONCEALMENT: unclear.

EPOC- QUALITY CRITERIA 2002: C.high risk of bias.

METHOD OF GENERATING RANDOMISATION SCHEDULE: not specified.

METHOD OF CONCEALMENT OF ALLOCATION: not specified.

BLINDING:

-PARTICIPANTS: no

-PROVIDER/S: no

.OUTCOME ASSESSOR/S: no

INTENTION TO TREAT ANALYSIS: not stated

BASELINE COMPARABILITY OF INTERVENTION AND CONTROL GROUPS: not stated.

STATISTICAL METHODS AND THEIR APPROPRIATENESS: t-test (probably).

CONSUMER INVOLVEMENT: not stated.

Participants

DESCRIPTION: surgery patients.

GEOGRAPHIC LOCATION: USA.

SETTING: discharged home from an acute care setting.

NUMBER OF PARTICIPANTS:

ELIGIBLE: 153 ?

RANDOMISED TO INTERVENTION: 78.

RANDOMISED TO CONTROL: 75 .

INCLUDED IN ANALYSIS INTERVENTION GROUP: 78.

INCLUDED IN ANALYSIS CONTROL GROUP: 75.

AGE: RANGE OR MEAN (SD): ?

GENDER (\% MALE): ?

ETHNICITY: unclear.

PRINCIPAL HEALTH PROBLEM OR DIAGNOSIS: unclear.

OTHER HEALTH PROBLEM/S: unclear.

TREATMENT RECEIVED/RECEIVING: surgery.

OTHER SOCIAL/DEMOGRAPHIC DETAILS:

Telephone follow-up, initiated by a hospital-based health professional, for postdischarge problems in patients discharged from hospital

Copyright $\odot 2010$ The Cochrane Collaboration. Published by John Wiley \& Sons, Ltd. 
Gombeski 1993 (Continued) Interventions
DETAILS OF INTERVENTION: Three weeks after discharge, the patient was called using an interview guide; the 3 week period was selected because it gives the surgical patient sufficient time to overcome most of the normal problems associated with any surgery; yet if problems persisted, they could be identified and addressed before the routine 6 weeks follow-up.

DETAILS OF CONTROL: usual care.

CO-INTERVENTION? unclear.

DETAILS OF CO-INTERVENTIONS: unclear.

DELIVERY OF INTERVENTION:

Frequency: 1.

First time at day 3 weeks after discharge.

Period:

PROVIDERS: someone who was not a nurse, but had worked in a number of hospital units and was familiar with hospital patient's concerns.

INTERVENTION QUALITY: unclear.

FIDELITY/INTEGRITY: unclear.

\section{NUMBER OF OUTCOMES: 1.}

OUTCOME / TOOL / TOOL VALIDATED / METHOD OF ASSESSMENT / TIME OF ASSESSMENT A.Psycho-social health of patients (uncertainty, anxiety, informational needs, mood, coping, quality of life, social activity, ...): Satisfaction / self-developed / no / written questionnaire / 6 weeks after discharge.

B.Physical health of patients (eg. functional status, self-care, self-efficacy, independence, ...):

C.Other consumer oriented outcomes (eg. treatment adherence, knowledge, adverse events, ..):

D. Health service delivery oriented outcomes (eg. hospital readmission, health services utilization, ..):

\begin{tabular}{ll}
\hline Notes & CHANGES IN TRIAL PROTOCOL \\
& $\cdot$ CONTACT WITH AUTHOR \\
& $\cdot$ POWER CALCULATION? \\
& $\cdot$ RECORD IF THE STUDY WAS TRANSLATED FROM A LANGUAGE OTHER THAN ENGLISH. \\
& $\cdot$ RECORD IF THE STUDY WAS A DUPLICATE PUBLICATION.
\end{tabular}

\section{Risk of bias}

\begin{tabular}{lll}
\hline Bias & Authors' judgement & Support for judgement \\
\hline Allocation concealment? & Unclear risk & B - Unclear \\
\hline
\end{tabular}

Gortner 1990

METhods
AIM OF INTERVENTION: The TFU was aimed to monitor recovery, to reinforce risk-factor reduction,
COach toward activity and to provide reassurance.
AIM OF STUDY: whether in-patient education and telephone monitoring during convalescence en-
hanced perceptions of cardiac efficacy and reported activity.

Telephone follow-up, initiated by a hospital-based health professional, for postdischarge problems in patients discharged from hospital 


\title{
STUDY DESIGN: RCT.
}

METHODS OF RECRUITMENT OF PARTICIPANTS: patient and their family members were approached the day before surgery and invited to participate in the study. Randomization occurred following transfer from the ICU and was carried out according to cluster randomized design.

INCLUSION CRITERIA FOR PARTICIPATION IN STUDY

first-time and repeat cardiac surgery patients between 30 and 75 years of age undergoing CABG or valve replacement

EXCLUSION CRITERIA FOR PARTICIPATION IN STUDY: not specified.

INFORMED CONSENT OBTAINED? yes.

ETHICAL APPROVAL? unclear.

FUNDING: unclear.

\section{ASSESSMENT OF STUDY QUALITY}

ALLOCATION CONCEALMENT: adequate.

EPOC- QUALITY CRITERIA 2002: C.high risk of bias.

METHOD OF GENERATING RANDOMISATION SCHEDULE: "Randomization occured following transfer from the intensive care unit and was carried out according to a cluster randomization design (Donner et al. 1981). In this procedure, a a group or cluster of subjects is formed and then is assigned as a group, using computer-generated random numbers, to experimental or control conditions. Cluster size was randomly determined, and usually was eight to ten patients...Random assignments were made in accordance with plans drawn by W. Hauck, the consulting statistician; the sequence of randomization was not revealed to research assistants." (p. 1134).

METHOD OF CONCEALMENT OF ALLOCATION: as above.

BLINDING:

-PARTICIPANTS: no

-PROVIDER/S: no

-OUTCOME ASSESSOR/S: no

INTENTION TO TREAT ANALYSIS: not stated

BASELINE COMPARABILITY OF INTERVENTION AND CONTROL GROUPS: yes

STATISTICAL METHODS AND THEIR APPROPRIATENESS:

t-test/ancova/multiple regression

CONSUMER INVOLVEMENT:

not stated

Participants

\author{
DESCRIPTION : cardiac patients / surgery patients. \\ GEOGRAPHIC LOCATION: USA. \\ SETTING: discharged home from an acute care setting. \\ NUMBER OF PARTICIPANTS: \\ ELIGIBLE: 156. \\ RANDOMISED TO INTERVENTION: 75. \\ RANDOMISED TO CONTROL: 77. \\ INCLUDED IN ANALYSIS INTERVENTION GROUP: 71. \\ INCLUDED IN ANALYSIS CONTROL GROUP: 77.
}

AGE: RANGE OR MEAN (SD): 58.

Telephone follow-up, initiated by a hospital-based health professional, for postdischarge problems in patients discharged from hospital 
Gortner 1990 (Continued)

GENDER (\% MALE): 80.

ETHNICITY: $42 \%$ caucasian,

PRINCIPAL HEALTH PROBLEM OR DIAGNOSIS: cardiovascular disease,

OTHER HEALTH PROBLEM/S: unclear.

TREATMENT RECEIVED/RECEIVING: CABG and/or valve replacement.

OTHER SOCIAL/DEMOGRAPHIC DETAILS:

Interventions

DETAILS OF INTERVENTION: Both groups were provided with routine information on recovery. The experimental group additionally received a slide programme and brief counseling session before discharge. After discharge the experimental group was followed by weekly telephones from a NURSE for 4 weeks and biweekly telephone between 4th and 8th week after discharge.

DETAILS OF CONTROL: usual care,

CO-INTERVENTION? yes.

DETAILS OF CO-INTERVENTIONS: routine information on recovery, consisting of a booklet and slide program.

DELIVERY OF INTERVENTION

Frequency: 6.

First time at day 1 week after discharge.

Period: 8 weeks.

PROVIDERS: nurse.

INTERVENTION QUALITY: poor since outcome assessment coincides with intervention.

FIDELITY/INTEGRITY: unclear.

Outcomes NUMBER OF OUTCOMES: 3

OUTCOME / TOOL / TOOL VALIDATED / METHOD OF ASSESSMENT / TIME OF ASSESSMENT

A.Psycho-social health of patients (uncertainty, anxiety, informational needs, mood, coping, quality of life, social activity, ..): Mood state / Profile of Mood States (McNair 1971) / yes / telephone interview / 4, 12 and 24 weeks after discharge.

B.Physical health of patients (eg. functional status, self-care, self-efficacy, independence, ..): Activity level / self-developed / no / patient's self-report during telephone interview / 4, 8, 12 and 24 weeks. Self-efficacy / Jenkins Self-Efficacy Scale (Jenkins 1988) / yes / telephone interview / at discharge, 4, 8, 12 and 24 weeks after discharge.

C.Other consumer oriented outcomes (e.g. treatment adherence, knowledge, adverse events, ..):

D. Health service delivery oriented outcomes (e.g. hospital readmission, health services utilization, ..):

Notes

- CHANGES IN TRIAL PROTOCOL

.CONTACT WITH AUTHOR: yes.

-POWER CALCULATION?

-RECORD IF THE STUDY WAS TRANSLATED FROM A LANGUAGE OTHER THAN ENGLISH.

·RECORD IF THE STUDY WAS A DUPLICATE PUBLICATION.

\section{Risk of bias}


Gortner 1990 (Continued)
Allocation concealment?
Low risk
A - Adequate

Hagopian 1990

Methods

DETAILS OF STUDY

AIM OF INTERVENTION: it was expected that patients who received a weekly telephone call would have less anxiety, less severe side effects, use more helpful self-care strategies and cope better than patients who did not receive TFU.

AIM OF STUDY: to investigate the effects that a weekly telephone call intervention had on patients' well-being.

STUDY DESIGN: CCT.

METHODS OF RECRUITMENT OF PARTICIPANTS: potential subjects from the physician's practice and meeting the study criteria were identified by the physician nurse team.

INCLUSION CRITERIA FOR PARTICIPATION IN STUDY: undergoing radiation therapy for cure /able to communicate by telephone.

EXCLUSION CRITERIA FOR PARTICIPATION IN STUDY: not specified.

INFORMED CONSENT OBTAINED? unclear.

ETHICAL APPROVAL? unclear.

FUNDING: yes.

ASSESSMENT OF STUDY QUALITY

ALLOCATION CONCEALMENT: unclear.

EPOC- QUALITY CRITERIA 2002: C.high risk of bias.

METHOD OF GENERATING RANDOMISATION SCHEDULE: not specified.

METHOD OF CONCEALMENT OF ALLOCATION: not specified.

BLINDING:

-PARTICIPANTS: no.

-PROVIDER/S: no.

.OUTCOME ASSESSOR/S: no.

INTENTION TO TREAT ANALYSIS: not stated.

BASELINE COMPARABILITY OF INTERVENTION AND CONTROL GROUPS: yes.

STATISTICAL METHODS AND THEIR APPROPRIATENESS: t-test/anova.

CONSUMER INVOLVEMENT: not stated.

Participants

DESCRIPTION: oncology patients

GEOGRAPHIC LOCATION: USA.

SETTING: discharged home from an acute care setting.

NUMBER OF PARTICIPANTS:

ELIGIBLE: 55.

RANDOMISED TO INTERVENTION: 27.

RANDOMISED TO CONTROL: 28 - - .

Telephone follow-up, initiated by a hospital-based health professional, for postdischarge problems in patients discharged from hospital

Copyright $\odot 2010$ The Cochrane Collaboration. Published by John Wiley \& Sons, Ltd. 
Hagopian 1990 (Continued)

INCLUDED IN ANALYSIS INTERVENTION GROUP: 27.

INCLUDED IN ANALYSIS CONTROL GROUP: 28 - - .

AGE: RANGE OR MEAN (SD): 58.

GENDER (\% MALE): 40.

ETHNICITY:

PRINCIPAL HEALTH PROBLEM OR DIAGNOSIS: cancer patients receiving radiotherapy (34\% breast cancer; plus 7 other types of cancer).

OTHER HEALTH PROBLEM/S: unclear.

TREATMENT RECEIVED/RECEIVING: radiotherapy.

OTHER SOCIAL/DEMOGRAPHIC DETAILS:

Interventions

DETAILS OF INTERVENTION: Experimental patients received usual care; in addition they received a weekly telephone call by NURSE to further assess problems and reinforce teaching. The weekly telephone calls continued until the first follow-up visit, which usually occurred 1 month after treatment was completed/

DETAILS OF CONTROL: usual care consisting of weekly on-treatment visits with both the physician and nurse during the course of the treatment, usually lasting 6 weeks.

CO-INTERVENTION? yes.

DETAILS OF CO-INTERVENTIONS: usual care consisting of weekly on-treatment visits with both the physician and nurse during the course of the treatment, usually lasting 6 weeks.

\section{DELIVERY OF INTERVENTION}

Frequency: 4.

First time at day 1 week after discharge.

Period: 4 weeks.

PROVIDERS: nurse.

INTERVENTION QUALITY: coincides with weekly treatment and counseling by nurse and physician.

FIDELITY/INTEGRITY: unclear.

Outcomes NUMBER OF OUTCOMES: 4

OUTCOME / TOOL / TOOL VALIDATED / METHOD OF ASSESSMENT / TIME OF ASSESSMENT

A.Psycho-social health of patients (uncertainty, anxiety, informational needs, mood, coping, quality of life, social activity, ...): Anxiety / state-anxiety inventory (Spielberger 1983) / yes / telephone interview / $1,2,3,4$ and 6 weeks after discharge. Coping / self-developed / adapted from the chronicity Impact and Coping instrument (Hymovich, 1984) / telephone interview / 1, 2, 3, 4 and 6 weeks after discharge.

B.Physical health of patients (eg. functional status, self-care, self-efficacy, independence, ..): Selfcare / self-developed / unclear / telephone interview / 1, 2, 3, 4 and 6 weeks after discharge

C.Other consumer oriented outcomes (eg. treatment adherence, knowledge, adverse events, ..): Sideeffects / self-developed: side-effects profile / unclear / telephone interview / 1, 2, 3, 4 and 6 weeks after discharge.

D. Health service delivery oriented outcomes (eg. hospital readmission, health services utilization, ...):

Notes $\quad$ CHANGES IN TRIAL PROTOCOL

.CONTACT WITH AUTHOR

-POWER CALCULATION? yes.

Telephone follow-up, initiated by a hospital-based health professional, for postdischarge problems in patients discharged from hospital 


\section{Risk of bias}

\begin{tabular}{lll}
\hline Bias & Authors' judgement & Support for judgement \\
\hline Allocation concealment? & Unclear risk & B - Unclear \\
\hline
\end{tabular}

\section{Hartford 2002}

\section{Methods}

DETAILS OF STUDY

AIM OF INTERVENTION: to reduce anxiety in patients and partners.

AIM OF STUDY: to determine the effectiveness of an information and support telephone intervention for reducing anxiety in patients who have undergone CABG surgery and their partners

STUDY DESIGN: RCT.

METHODS OF RECRUITMENT OF PARTICIPANTS: convenience sample.

INCLUSION CRITERIA FOR PARTICIPATION IN STUDY: first elective CABG without valve replacement/had a partner at home involved in their care/older than 18 years/able to understand and speak english/have a telephone/ able to hear telephone conversations.

EXCLUSION CRITERIA FOR PARTICIPATION IN STUDY: major comorbidity/psychiatric diagnosis/generalized anxiety or panic disorder.

INFORMED CONSENT OBTAINED? yes.

ETHICAL APPROVAL? yes.

FUNDING: unclear.

\section{ASSESSMENT OF STUDY QUALITY}

ALLOCATION CONCEALMENT: adequate.

EPOC- QUALITY CRITERIA 2002: B.moderate risk of bias.

METHOD OF GENERATING RANDOMISATION SCHEDULE: random number assignment and using opaque envelopes.

METHOD OF CONCEALMENT OF ALLOCATION: opaque envelopes.

BLINDING:

PARTICIPANTS: no.

-PROVIDER/S: no.

-OUTCOME ASSESSOR/S: yes.

INTENTION TO TREAT ANALYSIS: not stated.

BASELINE COMPARABILITY OF INTERVENTION AND CONTROL GROUPS: yes.

STATISTICAL METHODS AND THEIR APPROPRIATENESS: chi-square/anova/repeated measures analysis. CONSUMER INVOLVEMENT: not stated. 
Hartford 2002 (Continued)

\section{GEOGRAPHIC LOCATION: Canada.}

SETTING: discharged home from an acute care setting.

NUMBER OF PARTTICIPANTS:

ELIGIBLE: 166.

RANDOMISED TO INTERVENTION: 81.

RANDOMISED TO CONTROL: 68.

INCLUDED IN ANALYSIS INTERVENTION GROUP: 63.

INCLUDED IN ANALYSIS CONTROL GROUP: 68.

AGE: RANGE OR MEAN (SD): 63 (8).

GENDER (\% MALE): 86.

ETHNICITY: unclear.

PRINCIPAL HEALTH PROBLEM OR DIAGNOSIS: cardiovascular disease.

OTHER HEALTH PROBLEM/S: unclear.

TREATMENT RECEIVED/RECEIVING: CABG.

OTHER SOCIAL/DEMOGRAPHIC DETAILS: 98\% married, 50\% high school, 58\% retired. (and partners) in meeting their needs. Standardized protocols for predefined problems and concerns identified in the literature were developed. The intervention began on the day of discharge; this was followed by 6 telephone calls by NURSE on days 1, 2, 4, 7, 14 and 21 after discharge. The nurse was also on call 24 hours a day.

DETAILS OF CONTROL: usual care.

CO-INTERVENTION? no.

DETAILS OF CO-INTERVENTIONS:

DELIVERY OF INTERVENTION

Frequency: 6 ,

First time at day 1 day after discharge,

Period: 7 weeks,

PROVIDERS: nurse.

INTERVENTION QUALITY: unclear.

FIDELITY/INTEGRITY: unclear.

OUTCOME / TOOL / TOOL VALIDATED / METHOD OF ASSESSMENT / TIME OF ASSESSMENT

A.Psycho-social health of patients (uncertainty, anxiety, informational needs, mood, coping, quality of life, social activity, ..): Anxiety / Beck Anxiety Inventory (Beck, 1988) / yes / telephone interview / 2 days and 4 weeks and 8 weeks after discharge.

B.Physical health of patients (eg. functional status, self-care, self-efficacy, independence, ...):

C.Other consumer oriented outcomes (eg. treatment adherence, knowledge, adverse events, ..):

D. Health service delivery oriented outcomes (eg. hospital readmission, health services utilization, ...):

Notes

-CHANGES IN TRIAL PROTOCOL

.CONTACT WITH AUTHOR: yes.

Telephone follow-up, initiated by a hospital-based health professional, for postdischarge problems in patients discharged from hospital 
Hartford 2002 (Continued)

-POWER CALCULATION? yes.

-RECORD IF THE STUDY WAS TRANSLATED FROM A LANGUAGE OTHER THAN ENGLISH.

-RECORD IF THE STUDY WAS A DUPLICATE PUBLICATION.

\section{Risk of bias}

\begin{tabular}{lll}
\hline Bias & Authors' judgement & Support for judgement \\
\hline Allocation concealment? & Low risk & A - Adequate \\
\hline
\end{tabular}

\section{Jerant 2001}

Methods
DETAILS OF STUDY

AIM OF INTERVENTION: to reduce readmissions.

AIM OF STUDY: To compare the effectiveness of 3 hospital discharge care models for reduction congestive heart failure related readmission charges: 1 / home telecare delivered via a 2-way video-conference device with an integrated electronic stethoscope; 2/ nurse telephone calls; and 3/ usual outpatient care.

STUDY DESIGN: RCT.

METHODS OF RECRUITMENT OF PARTICIPANTS: During a one year period all patients with a primary admission diagnosis of chronic heart failure were screened on the inclusion criteria. Patients who agreed to participate were randomized before discharge, to one of 3 models, using sealed envelopes.

INCLUSION CRITERIA FOR PARTICIPATION IN STUDY: CHF/ aged 40 and older/ telephone at home/ English speaking/ area university of California/ adequate vision and hearing.

EXCLUSION CRITERIA FOR PARTICIPATION IN STUDY: to much comorbidity (Charlson score >6), Geriatric depression score $>7$, Mini mental state $<20$, symbol digit modalities test low.

INFORMED CONSENT OBTAINED? yes.

ETHICAL APPROVAL? yes.

FUNDING: yes.

ASSESSMENT OF STUDY QUALITY

ALLOCATION CONCEALMENT: adequate.

EPOC- QUALITY CRITERIA 2002: B. moderate risk of bias.

METHOD OF GENERATING RANDOMISATION SCHEDULE:random number assignment and using opaque envelopes.

METHOD OF CONCEALMENT OF ALLOCATION: opaque envelopes.

BLINDING:

-PARTICIPANTS: no.

-PROVIDER/S: no.

-OUTCOME ASSESSOR/S: no.

INTENTION TO TREAT ANALYSIS: yes.

BASELINE COMPARABILITY OF INTERVENTION AND CONTROL GROUPS: yes.

STATISTICAL METHODS AND THEIR APPROPRIATENESS: chi-square/anova. 
Jerant 2001 (Continued)

CONSUMER INVOLVEMENT: not stated.

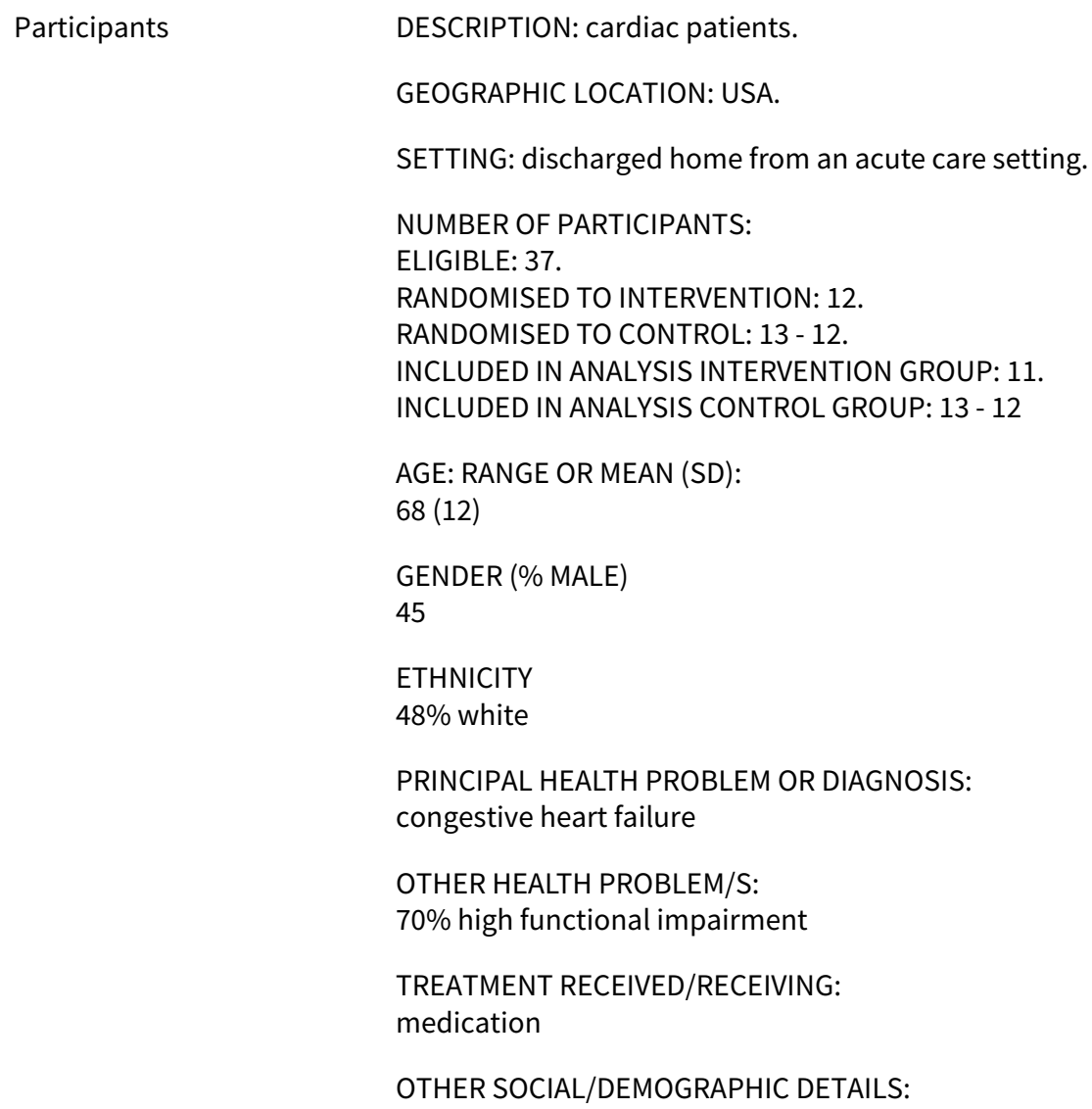

DETAILS OF INTERVENTION: All 3 groups received home visit of a nurse shortly after discharge and at day 60. Patient in telephone group received scheduled phone calls from nurse, whereas patients of the telecare group received scheduled telecare visits. Both groups also had possibility to contact study nurse. Difference between the intervention groups is that the telecare group could also see and not only hear study nurse, and vice versa. During contacts several health status measures were filled out.

DETAILS OF CONTROL: The telecare was instructed in the use of the equipment and received home telecare visits/ Patients in the usual care group received 2 home visits.

CO-INTERVENTION? yes.

DETAILS OF CO-INTERVENTIONS: all patients received an in-person home nurse visit shortly after discharge and second in-person home nurse visit 60 days later. During both visits completed some questionnaires.

\section{DELIVERY OF INTERVENTION}

Frequency: 6.

First time at day unclear after discharge.

Period: 8 weeks.

PROVIDERS: nurse.

INTERVENTION QUALITY: good.

FIDELITY/INTEGRITY: good. 
Jerant 2001 (Continued)

A.Psycho-social health of patients (uncertainty, anxiety, informational needs, mood, coping, quality of life, social activity, ...): Satisfaction / Client Satisfaction questionnaire (Attkisson, 1982) / yes / interview / 60 days. Mental status / SF-36 (Ware, 1992), Minnesota Living wit Heart Failure Questionnaire (Rector, 1992) / yes / interview / 60 days after discharge.

B.Physical health of patients (eg. functional status, self-care, self-efficacy, independence, ..): Physical status / SF-36 (Ware, 1992), Minnesota Living wit Heart Failure Questionnaire (Rector, 1992) / yes / interview / 60 days after discharge.

C.Other consumer oriented outcomes (eg. treatment adherence, knowledge, adverse events, ..):

D. Health service delivery oriented outcomes (eg. hospital readmission, health services utilization, ...):

Notes

-CHANGES IN TRIAL PROTOCOL: readmissions/ed-visits and charges were also measured but only at 6 months, which is outside the scope of this review.

·CONTACT WITH AUTHOR: yes.

-POWER CALCULATION? yes.

·RECORD IF THE STUDY WAS TRANSLATED FROM A LANGUAGE OTHER THAN ENGLISH.

·RECORD IF THE STUDY WAS A DUPLICATE PUBLICATION. Yes, see Jerant 2003.

\section{Risk of bias}

\begin{tabular}{lll}
\hline Bias & Authors' judgement & Support for judgement \\
\hline Allocation concealment? & Low risk & A - Adequate \\
\hline
\end{tabular}

Jones 1988

Methods

DETAILS OF STUDY

AIM OF INTERVENTION: To enhance compliance (based on health belief model)/

AIM OF STUDY: the purpose of the study was to test the effect of clinical and telephone intervention on compliance for ED-patients.

STUDY DESIGN: RCT.

METHODS OF RECRUITMENT OF PARTICIPANTS: consecutive patients who met the sampling criteria were randomly assigned to one of 4 groups.

INCLUSION CRITERIA FOR PARTICIPATION IN STUDY: Patients presenting at the ED with one of following problems (chest pain, hypertension, asthma, otitis media, diabetes, urinary tract infection, headache, urethritis, vaginitis, low back pain, rash)/ signed release of information/ able to respond to HBM intervention/ did not require hospital admission/ had a referral follow-up recommendation/ telephone at home.

EXCLUSION CRITERIA FOR PARTICIPATION IN STUDY: not specified.

INFORMED CONSENT OBTAINED? yes.

ETHICAL APPROVAL? yes.

FUNDING: yes.

ASSESSMENT OF STUDY QUALITY

ALLOCATION CONCEALMENT: unclear.

Telephone follow-up, initiated by a hospital-based health professional, for postdischarge problems in patients discharged from hospital 
EPOC- QUALITY CRITERIA 2002: C.high risk of bias.

METHOD OF GENERATING RANDOMISATION SCHEDULE: 2X2X11 factorial design/ blocked randomization within each presenting problem.

METHOD OF CONCEALMENT OF ALLOCATION: not specified.

BLINDING:

-PARTICIPANTS: no.

-PROVIDER/S: no.

-OUTCOME ASSESSOR/S: no.

INTENTION TO TREAT ANALYSIS: not stated.

BASELINE COMPARABILITY OF INTERVENTION AND CONTROL GROUPS: not stated.

STATISTICAL METHODS AND THEIR APPROPRIATENESS: chi-square/logistic regression.

CONSUMER INVOLVEMENT: not stated.

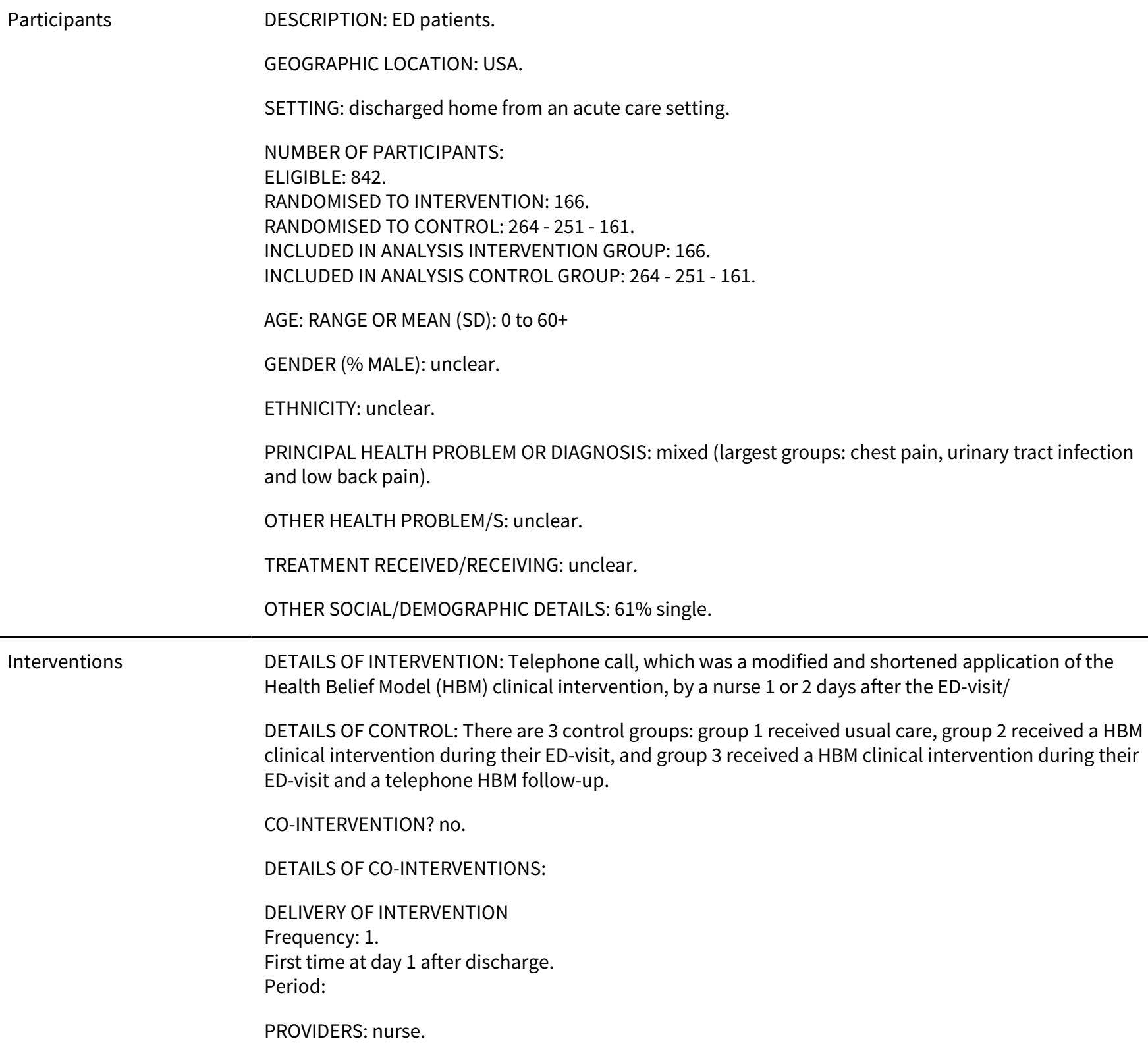
Health Belief Model (HBM) clinical intervention, by a nurse 1 or 2 days after the ED-visit/

DETAILS OF CONTROL: There are 3 control groups: group 1 received usual care, group 2 received a HBM clinical intervention during their ED-visit, and group 3 received a $\mathrm{HBM}$ clinical intervention during their ED-visit and a telephone HBM follow-up.

CO-INTERVENTION? no.

DETAILS OF CO-INTERVENTIONS:

DELIVERY OF INTERVENTION

Frequency: 1.

First time at day 1 after discharge.

Period:

PROVIDERS: nurse. 
INTERVENTION QUALITY: unclear.

FIDELITY/INTEGRITY: unclear.

NUMBER OF OUTCOMES: 1
OUTCOmes
A.Psycho-social health of patients (uncertainty, anxiety, informational needs, mood, coping, quality of
life, social activity, ..):
B.Physical health of patients (eg. functional status, self-care, self-efficacy, independence, ..):
C.Other consumer oriented outcomes (eg. treatment adherence, knowledge, adverse events, ..): Com-
pliance / self-developed / unclear / telephone interview to health agency where patient had referral /
different across patients.
D. Health service delivery oriented outcomes (eg. hospital readmission, health services utilization, ..):

Notes $\quad$ CHANGES IN TRIAL PROTOCOL

.CONTACT WITH AUTHOR

-POWER CALCULATION?

·RECORD IF THE STUDY WAS TRANSLATED FROM A LANGUAGE OTHER THAN ENGLISH.

-RECORD IF THE STUDY WAS A DUPLICATE PUBLICATION. yes, Jones et al. published 5 manuscripts, in which they present results for several subgroups (eg. hypertension, low back pain, otitis media, urinary tract infection).

\section{Risk of bias}

\begin{tabular}{lll}
\hline Bias & Authors' judgement & Support for judgement \\
\hline Allocation concealment? & Unclear risk & B - Unclear \\
\hline
\end{tabular}

Mohan 1999

AIM OF INTERVENTION: to enhance compliance with the use of the home apnea monitor for infants discharged on an apnea monitor.

AIM OF STUDY: this study was designed to test whether weekly telephone contact with a health professional would improve the use of the home apnea monitor.

STUDY DESIGN: RCT.

METHODS OF RECRUITMENT OF PARTICIPANTS: all infants discharged on apnea monitor during a 1.5 year period, were eligible for this study.

INCLUSION CRITERIA FOR PARTICIPATION IN STUDY: preterm infants with abnormal pneumocardiogram, patients with bronchopulmonary disease requiring oxygen support/ siblings of a sudden infant death syndrome victim, others infants with various pulmonary, cardiac or neurologic problems.

EXCLUSION CRITERIA FOR PARTICIPATION IN STUDY: not specified.

INFORMED CONSENT OBTAINED? yes.

ETHICAL APPROVAL? yes. 
Mohan 1999 (Continued)

FUNDING: unclear.

ASSESSMENT OF STUDY QUALITY

ALLOCATION CONCEALMENT: unclear.

EPOC- QUALITY CRITERIA 2002: C. high risk of bias.

METHOD OF GENERATING RANDOMISATION SCHEDULE: stratified balanced block technique.

METHOD OF CONCEALMENT OF ALLOCATION: unclear.

BLINDING:

-PARTICIPANTS: no.

-PROVIDER/S: no.

-OUTCOME ASSESSOR/S: yes.

INTENTION TO TREAT ANALYSIS: not stated.

BASELINE COMPARABILITY OF INTERVENTION AND CONTROL GROUPS: yes.

STATISTICAL METHODS AND THEIR APPROPRIATENESS: t-test/Mann-Whitney U-test/chi-square.

CONSUMER INVOLVEMENT: not stated.

Participants

DESCRIPTION: pediatric patients.

GEOGRAPHIC LOCATION: USA.

SETTING: discharged home from an acute care setting.

NUMBER OF PARTICIPANTS:

ELIGIBLE: 69

RANDOMISED TO INTERVENTION: 30.

RANDOMISED TO CONTROL: 32.

INCLUDED IN ANALYSIS INTERVENTION GROUP: 30.

INCLUDED IN ANALYSIS CONTROL GROUP: 32

AGE: RANGE OR MEAN (SD): 0.5 in infants/ 25 years in mothers.

GENDER (\% MALE): not stated.

ETHNICITY: not stated.

PRINCIPAL HEALTH PROBLEM OR DIAGNOSIS: preterm infants with abnormal pneumocardiogram, patients with bronchopulmonary disease requiring oxygen support/ siblings of a sudden infant death syndrome victim, others infants with various pulmonary, cardiac or neurologic problems.

OTHER HEALTH PROBLEM/S: unclear.

TREATMENT RECEIVED/RECEIVING:

OTHER SOCIAL/DEMOGRAPHIC DETAILS: $50 \%$ of mothers married.

DETAILS OF INTERVENTION: above the care comparable to the control group, patients in the experimental group received an additional phone call consisting of a structured interview every week for a total of 8 weeks. The questionnaire addressed the use of monitors and the well-being of the baby, including any need for an office visit or hospitalisation.

DETAILS OF CONTROL: care for all groups: access to a physician in the neonatal intensive care unit at all times, initial instruction as well as support 24 hours per day from the monitor vending company, follow-up visits with a neonatologist or pediatrician within 2 weeks of discharge and about every month for the next 3 months and most infants had 1-3 visits by a home nurse in the first 2 weeks following discharge.

Telephone follow-up, initiated by a hospital-based health professional, for postdischarge problems in patients discharged from hospital 
Mohan 1999 (Continued)

\section{CO-INTERVENTION? yes.}

DETAILS OF CO-INTERVENTIONS: see control intervention.

DELIVERY OF INTERVENTION:

Frequency: 8.

First time at day 7 after discharge.

Period: 8 weeks.

PROVIDERS: unclear, probably physician.

INTERVENTION QUALITY: unclear.

FIDELITY/INTEGRITY: unclear.

Outcomes

NUMBER OF OUTCOMES: 2

OUTCOME / TOOL / TOOL VALIDATED / METHOD OF ASSESSMENT / TIME OF ASSESSMENT

A.Psycho-social health of patients (uncertainty, anxiety, informational needs, mood, coping, quality of life, social activity, ..): Satisfaction / self-developed / partly, adopted from Gerard and Peterson's cardiac patient learning needs inventory / unclear / unclear, but in first month after discharge.

B.Physical health of patients (eg. functional status, self-care, self-efficacy, independence, ...):

C.Other consumer oriented outcomes (eg. treatment adherence, knowledge, adverse events, ..): Knowledge / self-developed: heart disease management questionnaire / partly; Kuder-Richardson coefficient 0.36 / unclear / unclear, but in first month after discharge.

D. Health service delivery oriented outcomes (eg. hospital readmission, health services utilization, ..):

Notes
$\cdot$ CHANGES IN TRIAL PROTOCOL
$\cdot$ CONTACT WITH AUTHOR
$\cdot$ POWER CALCULATION? yes.
$\cdot$ RECORD IF THE STUDY WAS TRANSLATED FROM A LANGUAGE OTHER THAN ENGLISH.
$\cdot$ RECORD IF THE STUDY WAS A DUPLICATE PUBLICATION.

\section{Risk of bias}

\begin{tabular}{lll}
\hline Bias & Authors' judgement & Support for judgement \\
\hline Allocation concealment? & Unclear risk & B - Unclear \\
\hline
\end{tabular}

Munro 1994

AIM OF INTERVENTION: to enhance support.

AIM OF STUDY: the hypothesis tested was that routine contact by telephone might significantly improve the adequacy of support for patients during the potentially stressful period between completing radiotherapy and the first follow-up visit.

\section{STUDY DESIGN: CCT.}

METHODS OF RECRUITMENT OF PARTICIPANTS: consecutive unselected outpatients attending for radiotherapy. 
INCLUSION CRITERIA FOR PARTICIPATION IN STUDY: radiotherapy patients.

EXCLUSION CRITERIA FOR PARTICIPATION IN STUDY: did not understand English/ not having a telephone/ HIV-related malignancy/ less than 5 dose of radiotherapy/ inhospital patients.

INFORMED CONSENT OBTAINED? unclear.

ETHICAL APPROVAL? unclear.

FUNDING: unclear.

ASSESSMENT OF STUDY QUALITY

ALLOCATION CONCEALMENT: unclear.

EPOC- QUALITY CRITERIA 2002: C.high risk of bias.

METHOD OF GENERATING RANDOMISATION SCHEDULE: unclear.

METHOD OF CONCEALMENT OF ALLOCATION: unclear.

BLINDING:

-PARTICIPANTS: no.

-PROVIDER/S: no.

.OUTCOME ASSESSOR/S: no.

INTENTION TO TREAT ANALYSIS: yes.

BASELINE COMPARABILITY OF INTERVENTION AND CONTROL GROUPS: not stated.

STATISTICAL METHODS AND THEIR APPROPRIATENESS: chi-square.

CONSUMER INVOLVEMENT: not stated.

\section{Participants} DESCRIPTION: oncology patients.

GEOGRAPHIC LOCATION: UK.

SETTING: discharged home from an acute care setting.

NUMBER OF PARTICIPANTS:

ELIGIBLE: 100.

RANDOMISED TO INTERVENTION: 49.

RANDOMISED TO CONTROL: 39.

INCLUDED IN ANALYSIS INTERVENTION GROUP: 33.

INCLUDED IN ANALYSIS CONTROL GROUP: 39.

AGE: RANGE OR MEAN (SD): 30 to 88.

GENDER (\% MALE): 42.

ETHNICITY: not stated.

PRINCIPAL HEALTH PROBLEM OR DIAGNOSIS: radiotherapy for cancer (breast 43\%, lung, 31\%,..).

OTHER HEALTH PROBLEM/S: unclear.

TREATMENT RECEIVED/RECEIVING:

OTHER SOCIAL/DEMOGRAPHIC DETAILS: and 18 after completing radiotherapy; the telephone calls were made by a member of staff (radiographer, nurse, or doctor who was known to the patient). The calls were semistructured, the questions to be asked being: 'how are you feeling?', 'are you having any problems?', 'have you any further side-effects 
Munro 1994 (Continued)

from treatment?', 'do you need to make an appointment ..?'. patients were asked if they had any additional worries or concerns. wherever possible action was taken.

DETAILS OF CONTROL: all patients were seen once a week in the clinic by a doctor during the radiotherapy treatment. Additional advice and support was given, where necessary, by radiographers and nurses. In the usual care group no attempt was made to contact the patients between completing treatment and the first follow-up visit. If patients telephoned the department for advice or support this was provided.

CO-INTERVENTION? yes.

DETAILS OF CO-INTERVENTIONS: see control intervention.

DELIVERY OF INTERVENTION

Frequency: 4.

First time at day 4 after discharge.

Period: 3 weeks.

PROVIDERS: mixed (nurse, radiographer, doctor).

INTERVENTION QUALITY: unclear.

FIDELITY/INTEGRITY: unclear.

\section{Outcomes}

\section{NUMBER OF OUTCOMES: 1}

OUTCOME / TOOL / TOOL VALIDATED / METHOD OF ASSESSMENT / TIME OF ASSESSMENT

A.Psycho-social health of patients (uncertainty, anxiety, informational needs, mood, coping, quality of life, social activity, ...): Satisfaction (adequacy of support) / self-developed / no / questionnaire / 4 weeks after discharge.

B.Physical health of patients (eg. functional status, self-care, self-efficacy, independence, ...):

C.Other consumer oriented outcomes (eg. treatment adherence, knowledge, adverse events, ...):

D. Health service delivery oriented outcomes (eg. hospital readmission, health services utilization, ..):

$\begin{array}{ll}\text { Notes } & \cdot \text { CHANGES IN TRIAL PROTOCOL } \\ & \cdot \text { CONTACT WITH AUTHOR } \\ & \cdot \text { POWER CALCULATION? YeS. } \\ & \cdot \text { RECORD IF THE STUDY WAS TRANSLATED FROM A LANGUAGE OTHER THAN ENGLISH. } \\ & \cdot \text { RECORD IF THE STUDY WAS A DUPLICATE PUBLICATION. }\end{array}$

•RECORD IF THE STUDY WAS A DUPLICATE PUBLICATION.

\section{Risk of bias}

\begin{tabular}{lll}
\hline Bias & Authors' judgement & Support for judgement \\
\hline Allocation concealment? & Unclear risk & B - Unclear \\
\hline
\end{tabular}

Nelson 1991

Methods DETAILS OF STUDY

AIM OF INTERVENTION: improve the appropriateness of the parents use of follow-up care.

AIM OF STUDY: To test the hypothesis that the appropriateness of parents' use of early follow-up care after ED visits can be improved by post visit support from a nurse practitioner. We hypothesized that

Telephone follow-up, initiated by a hospital-based health professional, for postdischarge problems in patients discharged from hospital 
Nelson 1991 (Continued)

telephone support, given by pediatric nurse practitioners to parents within 1 day after their ED-visit for their children's acute illness could improve the appropriateness of the parents use of follow-up care.

STUDY DESIGN: RCT.

METHODS OF RECRUITMENT OF PARTICIPANTS: potential subjects were identified on arrival at the ED. Patients were told that they were conducting a survey to try to learn ways of improving pediatric ED care.

INCLUSION CRITERIA FOR PARTICIPATION IN STUDY: child younger than 8 years accompanied by parent or usual caretaker, free of active chronic illness, presenting with a chief complaint suggesting an acute infectious or allergic condition/ parents speaking English, acces to telephone, primary care source is hospital's primary care center.

EXCLUSION CRITERIA FOR PARTICIPATION IN STUDY: requiring hospital admission.

INFORMED CONSENT OBTAINED? yes.

ETHICAL APPROVAL? yes.

FUNDING: yes.

ASSESSMENT OF STUDY QUALITY

ALLOCATION CONCEALMENT: adequate.

EPOC- QUALITY CRITERIA 2002: B. moderate risk of bias.

METHOD OF GENERATING RANDOMISATION SCHEDULE: use of a random number table and a balanced block-randomization

METHOD OF CONCEALMENT OF ALLOCATION: sealed envelopes given to the parents on leaving the ED.

BLINDING:

-PARTICIPANTS: no.

-PROVIDER/S: no.

-OUTCOME ASSESSOR/S: yes.

INTENTION TO TREAT ANALYSIS: yes.

BASELINE COMPARABILITY OF INTERVENTION AND CONTROL GROUPS: yes.

STATISTICAL METHODS AND THEIR APPROPRIATENESS: chi-square.

CONSUMER INVOLVEMENT: not stated.

Participants 
Nelson 1991 (Continued)

\section{ETHNICITY \\ $77 \%$ black}

PRINCIPAL HEALTH PROBLEM OR DIAGNOSIS:

ED-visit with complaint of infectious or allergic condition

OTHER HEALTH PROBLEM/S:

unclear

TREATMENT RECEIVED/RECEIVING:

antibiotic in $46 \%$

OTHER SOCIAL/DEMOGRAPHIC DETAILS:

parents $86 \%$ single and $74 \%$ unemployed

Interventions

DETAILS OF INTERVENTION: The intervention consisted of only a single telephone call; mostly the call required less than 5 minutes. The NP called each parent in 6 to 18 hours after discharge from the ED. She offered further explanation about the child's diagnosis and treatment, reinforced follow-up instructions and offered around the clock access to herself or another NP by telephone if needed; the protocol allowed her to answer questions or offer clinical assistance over the phone if it seemed warranted.

DETAILS OF CONTROL: usual care.

CO-INTERVENTION? no.

DETAILS OF CO-INTERVENTIONS:

DELIVERY OF INTERVENTION

Frequency: 1.

First time at day 1 after discharge.

Period:

PROVIDERS: nurse.

INTERVENTION QUALITY: unclear.

FIDELITY/INTEGRITY: unclear.

Outcomes NUMBER OF OUTCOMES: 1

OUTCOME / TOOL / TOOL VALIDATED / METHOD OF ASSESSMENT / TIME OF ASSESSMENT

A.Psycho-social health of patients (uncertainty, anxiety, informational needs, mood, coping, quality of life, social activity, ..):

B.Physical health of patients (eg. functional status, self-care, self-efficacy, independence, ...):

C.Other consumer oriented outcomes (eg. treatment adherence, knowledge, adverse events, ...): Compliance (appropriate use of follow-up care) / self-developed / yes / hospital record/ telephone interview / 1 week after discharge

D. Health service delivery oriented outcomes (eg. hospital readmission, health services utilization, ..):

Notes $\quad$ CHANGES IN TRIAL PROTOCOL

-CONTACT WITH AUTHOR

-POWER CALCULATION?

·RECORD IF THE STUDY WAS TRANSLATED FROM A LANGUAGE OTHER THAN ENGLISH.

-RECORD IF THE STUDY WAS A DUPLICATE PUBLICATION.

\section{Risk of bias}

Telephone follow-up, initiated by a hospital-based health professional, for postdischarge problems in patients discharged from hospital 76 to home (Review)

Copyright $\odot 2010$ The Cochrane Collaboration. Published by John Wiley \& Sons, Ltd. 
Nelson 1991 (Continued)

Bias Authors' judgement Support for judgement

Allocation concealment? Low risk A-Adequate

Ouellet 2003

Methods
DETAILS OF STUDY

AIM OF INTERVENTION: to help ease surgical orthopaedic patients' transition from hospital to home and to identify problems associated with this transition.

AIM OF STUDY: this was a pilot study designed to explore the effectiveness of a post-discharge telephone call for surgical orthopaedic patients; the focus of the study was to identify and resolve problems associated with the study protocol and the data collection tools.

\section{STUDY DESIGN: CCT.}

METHODS OF RECRUITMENT OF PARTICIPANTS: the sample was drawn from a pool of adult patients admitted to the orthopaedic unit for either elective or emergency orthopaedic surgery during a 3 month period. Prospective participants were identified through the use of posters placed in patients' rooms. Patients who expressed an interest in the study were approached prior to their discharge by a research assistant who explained the purpose of the study.

INCLUSION CRITERIA FOR PARTICIPATION IN STUDY: English speaking/ 17 years and older/ discharged to a private residence with phone.

EXCLUSION CRITERIA FOR PARTICIPATION IN STUDY: not specified.

INFORMED CONSENT OBTAINED? yes.

ETHICAL APPROVAL? unclear.

FUNDING: unclear.

ASSESSMENT OF STUDY QUALITY

ALLOCATION CONCEALMENT: unclear.

EPOC- QUALITY CRITERIA 2002: C. high risk of bias.

METHOD OF GENERATING RANDOMISATION SCHEDULE: unclear.

METHOD OF CONCEALMENT OF ALLOCATION: not specified.

BLINDING:

-PARTICIPANTS: no.

-PROVIDER/S: no.

-OUTCOME ASSESSOR/S: yes.

INTENTION TO TREAT ANALYSIS: not stated.

BASELINE COMPARABILITY OF INTERVENTION AND CONTROL GROUPS: yes.

STATISTICAL METHODS AND THEIR APPROPRIATENESS: not stated.

CONSUMER INVOLVEMENT: not stated.
DESCRIPTION: surgery patients.

GEOGRAPHIC LOCATION: Canada.

SETTING: discharged home from an acute care setting. 
Ouellet 2003 (Continued)

\author{
NUMBER OF PARTTICIPANTS: \\ ELIGIBLE: 60. \\ RANDOMISED TO INTERVENTION: 27. \\ RANDOMISED TO CONTROL: 26. \\ INCLUDED IN ANALYSIS INTERVENTION GROUP: 27.
}

INCLUDED IN ANALYSIS CONTROL GROUP: 26.

AGE: RANGE OR MEAN (SD): 56.8 (17.6).

GENDER (\% MALE): 55.

ETHNICITY: not stated.

PRINCIPAL HEALTH PROBLEM OR DIAGNOSIS: orthopedic problem.

OTHER HEALTH PROBLEM/S: unclear.

TREATMENT RECEIVED/RECEIVING: orthopedic surgery (68\% elective, 32\% emergency).

OTHER SOCIAL/DEMOGRAPHIC DETAILS: 69\% high school or more.

DETAILS OF INTERVENTION: The intervention consisted of a follow-up call made by the unit manager (=nurse), or her designate, 24 to 72 hours post discharge. Information obtained was recorded on a form which consisted of a checklist of specific concerns/problems often encountered by post-op patients and a list of relevant nursing interventions in addition to information about the call.

DETAILS OF CONTROL: usual care.

CO-INTERVENTION? no.

DETAILS OF CO-INTERVENTIONS:

DELIVERY OF INTERVENTION:

Frequency: 1.

First time at day 1-3 after discharge.

Period:

PROVIDERS: nurse.

INTERVENTION QUALITY: length of intervention between 1-25 minutes.

FIDELITY/INTEGRITY: unclear.

\title{
Outcomes
}

\section{NUMBER OF OUTCOMES: 3}

OUTCOME / TOOL / TOOL VALIDATED / METHOD OF ASSESSMENT / TIME OF ASSESSMENT

A.Psycho-social health of patients (uncertainty, anxiety, informational needs, mood, coping, quality of life, social activity, ...):

B.Physical health of patients (eg. functional status, self-care, self-efficacy, independence, ..):Symptoms / self-developed / yes / telephone interview / 4 weeks after discharge. Recovery / self-developed / yes / telephone interview / 4 weeks after discharge.

C.Other consumer oriented outcomes (eg. treatment adherence, knowledge, adverse events, ...):

D. Health service delivery oriented outcomes (eg. hospital readmission, health services utilization, ..): Readmission / self-developed / yes / telephone interview / 4 weeks after discharge. ED-visits. 
Ouellet 2003 (Continued)

·RECORD IF THE STUDY WAS A DUPLICATE PUBLICATION.

\section{Risk of bias}

\begin{tabular}{lll}
\hline Bias & Authors' judgement & Support for judgement \\
\hline Allocation concealment? & Unclear risk & B - Unclear \\
\hline
\end{tabular}

Phillips 1999

\section{Methods}

DETAILS OF STUDY

AIM OF INTERVENTION: telehealth interventions offer a potentially promising way to improve care and continue patient education once patients have returned to the community/

AIM OF STUDY: to determine which of 3 approaches to care produces the lowest incidence or pressure ulcers, promotes the most effective care of sores that develop and leads to the fewest hospitalizations in newly injured patients with spinal cord injury after discharge.

STUDY DESIGN: CCT.

METHODS OF RECRUITMENT OF PARTICIPANTS: During a 6 month period, patients with newly injured spinal cords were recruited. Patients for the video group were first recruited; those qualifying were informed about the study and asked to volunteer; following recruitment of the video group, matched controls (age, race, severity of injury) were recruited for the telephone group and the standard care group.

INCLUSION CRITERIA FOR PARTICIPATION IN STUDY: scheduled for discharge to the community/ have telephone at home and for the video group living in Georgia.

EXCLUSION CRITERIA FOR PARTICIPATION IN STUDY: not specified.

INFORMED CONSENT OBTAINED? yes

ETHICAL APPROVAL? unclear.

FUNDING: unclear.

ASSESSMENT OF STUDY QUALITY

ALLOCATION CONCEALMENT: inadequate.

EPOC- QUALITY CRITERIA 2002: C.high risk of bias.

METHOD OF GENERATING RANDOMISATION SCHEDULE:not done.

METHOD OF CONCEALMENT OF ALLOCATION: not specified.

BLINDING:

-PARTICIPANTS: no.

-PROVIDER/S: no.

-OUTCOME ASSESSORS: not clear.

INTENTION TO TREAT ANALYSIS: not stated.

BASELINE COMPARABILITY OF INTERVENTION AND CONTROL GROUPS: yes.

STATISTICAL METHODS AND THEIR APPROPRIATENESS: kruskall-wallis test/ chi-square.

CONSUMER INVOLVEMENT: not stated.

Participants DESCRIPTION: neurological patients.

Telephone follow-up, initiated by a hospital-based health professional, for postdischarge problems in patients discharged from hospital 79 to home (Review)

Copyright @ 2010 The Cochrane Collaboration. Published by John Wiley \& Sons, Ltd. 
Phillips 1999 (Continued)

GEOGRAPHIC LOCATION: USA.

SETTING: discharged home from an acute care setting.

NUMBER OF PARTICIPANTS:

ELIGIBLE: 37.

RANDOMISED TO INTERVENTION: 14.

RANDOMISED TO CONTROL: 12 - 11.

INCLUDED IN ANALYSIS INTERVENTION GROUP: 13

INCLUDED IN ANALYSIS CONTROL GROUP: 12 - 10.

AGE: RANGE OR MEAN (SD): 33 (12).

GENDER (\% MALE): 74.

ETHNICITY: 31\% African American.

PRINCIPAL HEALTH PROBLEM OR DIAGNOSIS: new spinal cord injury.

OTHER HEALTH PROBLEM/S: unclear.

TREATMENT RECEIVED/RECEIVING: unclear.

OTHER SOCIAL/DEMOGRAPHIC DETAILS: 46\% married; $91 \%$ employed; $48 \%$ post high school education; slight differences in video group.

Interventions

DETAILS OF INTERVENTION: both the video and the telephone group received weekly interventions for 10-12 weeks. The telephone group participated in telephone only counseling sessions for approximately 10 weeks after discharge. During the telephone sessions patients were guided through skin checkups and were also assisted in problem solving related to bowel, diet or any matter of concern.

DETAILS OF CONTROL: The videogroup received weekly interventions for approximately. 10-12 weeks. In the video group, patients participated in weekly counseling sessions using the video-unit for the first 6-8 weeks following discharge; through the images generated the nurse was able to check visually the condition of the patients skin and to monitor him for ulcers; through visual contact the nurse could also help resolve problems related to wheelchairs, mattresses and mobility about the house. Following the video-sessions, patients then received weekly telephone counseling for approximately. 4-6 weeks. The standard care group was given instruction on using the centers' help line; this line provides information and counseling free of charge to patients who call the center on their own initiative.

CO-INTERVENTION? no.

DETAILS OF CO-INTERVENTIONS:

DELIVERY OF INTERVENTION

Frequency: 12.

First time at day 7 after discharge.

Period: 12 weeks.

PROVIDERS: nurse.

INTERVENTION QUALITY: unclear.

FIDELITY/INTEGRITY: unclear.

Outcomes

NUMBER OF OUTCOMES: 2

OUTCOME / TOOL / TOOL VALIDATED / METHOD OF ASSESSMENT / TIME OF ASSESSMENT

A.Psycho-social health of patients (uncertainty, anxiety, informational needs, mood, coping, quality of life, social activity, ..):

B.Physical health of patients (eg. functional status, self-care, self-efficacy, independence, ..): Tracking/diagnosing pressure ulcer / self-developed / no / unclear / every 8-12 weeks but only reported for a 1 year period (therefore not included in the analysis for this review).

Telephone follow-up, initiated by a hospital-based health professional, for postdischarge problems in patients discharged from hospital

Copyright @ 2010 The Cochrane Collaboration. Published by John Wiley \& Sons, Ltd. 
D. Health service delivery oriented outcomes (eg. hospital readmission, health services utilization, ...): Readmission / unclear / unclear / unclear / at 1 year (not included therefore in review data-analysis)

Notes

-CHANGES IN TRIAL PROTOCOL

-CONTACT WITH AUTHOR

-POWER CALCULATION? yes.

-RECORD IF THE STUDY WAS TRANSLATED FROM A LANGUAGE OTHER THAN ENGLISH.

-RECORD IF THE STUDY WAS A DUPLICATE PUBLICATION.

\section{Risk of bias}

\begin{tabular}{lll}
\hline Bias & Authors' judgement & Support for judgement \\
\hline Allocation concealment? & High risk & C - Inadequate \\
\hline
\end{tabular}

Phillips 2001

\section{Methods}

\section{DETAILS OF STUDY}

AIM OF INTERVENTION: to reduce the incidence of secondary conditions among people with mobility impairment resulting from spinal cord injury.

AIM OF STUDY: the results are presented on health related outcomes of a randomized trial of telehealth interventions to reduce the incidence of secondary conditions among people with mobility impairment resulting from spinal cord injury.

\section{STUDY DESIGN: CCT.}

METHODS OF RECRUITMENT OF PARTICIPANTS: patients with spinal cord injury were recruited during their initial stay. Any patient from 18 to 60 years of age with a newly acquired spinal cord injury was eligible. All participants were research volunteers; once they agreed to participate they were randomly assigned to 1 of 3 groups.

INCLUSION CRITERIA FOR PARTICIPATION IN STUDY: new spinal cord injury/age 18-60/ have telephone/ discharged to the community.

EXCLUSION CRITERIA FOR PARTICIPATION IN STUDY: concomitant diagnosis of brain injury, known active substance abuse, mobility impairment level mild.

INFORMED CONSENT OBTAINED? yes.

ETHICAL APPROVAL? yes.

FUNDING: unclear.

ASSESSMENT OF STUDY QUALITY

ALLOCATION CONCEALMENT: unclear.

EPOC- QUALITY CRITERIA 2002: C. high risk of bias.

METHOD OF GENERATING RANDOMISATION SCHEDULE: unclear.

METHOD OF CONCEALMENT OF ALLOCATION: not specified.

BLINDING: 
Phillips 2001 (Continued)

-PARTICIPANTS: no.

-PROVIDER/S: no.

-OUTCOME ASSESSOR/S: yes.

INTENTION TO TREAT ANALYSIS: not stated.

BASELINE COMPARABILITY OF INTERVENTION AND CONTROL GROUPS: yes.

STATISTICAL METHODS AND THEIR APPROPRIATENESS: kruskall-wallis test.

CONSUMER INVOLVEMENT: not stated.

Participants

DESCRIPTION: neurological patients.

GEOGRAPHIC LOCATION: USA.

SETTING: discharged home from an acute care setting.

NUMBER OF PARTICIPANTS:

ELIGIBLE: unclear.

RANDOMISED TO INTERVENTION: 36.

RANDOMISED TO CONTROL: 36 - 39.

INCLUDED IN ANALYSIS INTERVENTION GROUP: 36.

INCLUDED IN ANALYSIS CONTROL GROUP: 36 - 39.

AGE: RANGE OR MEAN (SD): 37 (12).

GENDER (\% MALE): 77.

ETHNICITY: 17\% African American.

PRINCIPAL HEALTH PROBLEM OR DIAGNOSIS:new spinal cord injury.

OTHER HEALTH PROBLEM/S: unclear.

TREATMENT RECEIVED/RECEIVING: unclear.

OTHER SOCIAL/DEMOGRAPHIC DETAILS: 55\% married.

Interventions

DETAILS OF INTERVENTION: 3 groups: telephone, videophone and standard care. Video and phone groups took part in individual educational rehabilitation sessions with a nurse once a week for 5 weeks, then once every 2 weeks for one month. These sessions were in addition to any other regularly scheduled care, such as the two month post discharge care. The content and structure of the education sessions for the phone and video group were similar, except that the video group also saw real time images of the nurse. The intervention sessions lasted a total of nine weeks.

DETAILS OF CONTROL: 2 groups: video group and standard care. The standard care group were offered the routine care, which requires patients to call the hospital help line if and when they need assistance prior to the regular 2 months post discharge visit.

CO-INTERVENTION? no.

DETAILS OF CO-INTERVENTIONS:

DELIVERY OF INTERVENTION:

Frequency: 7.

First time at day 7 after discharge.

Period: 9 weeks.

PROVIDERS: nurse.

INTERVENTION QUALITY: unclear.

FIDELITY/INTEGRITY: unclear.

Telephone follow-up, initiated by a hospital-based health professional, for postdischarge problems in patients discharged from hospital

Copyright $\odot 2010$ The Cochrane Collaboration. Published by John Wiley \& Sons, Ltd. 
Phillips 2001 (Continued)

\section{Outcomes}

\section{NUMBER OF OUTCOMES: 4}

OUTCOME / TOOL / TOOL VALIDATED / METHOD OF ASSESSMENT / TIME OF ASSESSMENT

A.Psycho-social health of patients (uncertainty, anxiety, informational needs, mood, coping, quality of life, social activity, ..): Quality of life / quality of well-being scale (self-developed??) / unclear / interview / 5 and 9 weeks after discharge. Depression / Center for epidemiologic studies depression scale (self-developed?) / unclear / interview / 9 weeks after discharge.

B.Physical health of patients (eg. functional status, self-care, self-efficacy, independence, ...):

C.Other consumer oriented outcomes (eg. treatment adherence, knowledge, adverse events, ..):

D. Health service delivery oriented outcomes (eg. hospital readmission, health services utilization, ..): Readmission / unclear / unclear / unclear / at 1 year (not included therefore in review data-analysis).

\section{Notes}

-CHANGES IN TRIAL PROTOCOL

-CONTACT WITH AUTHOR

-POWER CALCULATION? yes.

·RECORD IF THE STUDY WAS TRANSLATED FROM A LANGUAGE OTHER THAN ENGLISH.

-RECORD IF THE STUDY WAS A DUPLICATE PUBLICATION.

\section{Risk of bias}

\section{Bias}

Authors' judgement Support for judgement

Allocation concealment? Unclear risk B-Unclear

Riegel 2002

Methods

\section{DETAILS OF STUDY}

AIM OF INTERVENTION: to reduce resource use (readmissions, hospital days, costs).

AIM OF STUDY: to assess the effectiveness of a standardized telephonic case-management intervention in decreasing resource use in patients with chronic heart failure.

\section{STUDY DESIGN: CCT.}

METHODS OF RECRUITMENT OF PARTICIPANTS: physicians known to admit patients with heart failure were matched by specialty (cardiology, internal medicine), practice size and number of HF admissions in the prior year. After matching, physicians were randomized to the intervention or usual care group. Although it was the physicians who were randomized, patients were the unit of analysis for this study. It was not feasible to randomize patients in the same physician practice to different groups because of the possibility that the physicians would modify care in the control group to mimic aspects of the intervention. Physicians were not informed of the group to which they were assigned. A total of 281 physicians were randomized.

INCLUSION CRITERIA FOR PARTICIPATION IN STUDY: confirmed diagnosis of heart failure as the primary reason for their hospital visit/ speak English or Spanish.

EXCLUSION CRITERIA FOR PARTICIPATION IN STUDY: cognitive impairment or psychiatric illness/ severe renal failure requiring dialysis/ terminal disease// discharge to a long term care setting/ previous enrolment in a HF disease program.

INFORMED CONSENT OBTAINED? yes.

ETHICAL APPROVAL? yes.

Telephone follow-up, initiated by a hospital-based health professional, for postdischarge problems in patients discharged from hospital 
Riegel 2002 (Continued)

FUNDING: yes.

ASSESSMENT OF STUDY QUALITY

ALLOCATION CONCEALMENT: unclear.

EPOC- QUALITY CRITERIA 2002: C.high risk of bias.

METHOD OF GENERATING RANDOMISATION SCHEDULE: unclear.

METHOD OF CONCEALMENT OF ALLOCATION: not specified.

BLINDING:

-PARTICIPANTS: no.

.PROVIDER/S: no.

-OUTCOME ASSESSOR/S: no.

INTENTION TO TREAT ANALYSIS: not stated.

BASELINE COMPARABILITY OF INTERVENTION AND CONTROL GROUPS: no.

STATISTICAL METHODS AND THEIR APPROPRIATENESS: anova/logistic regression/linear regression.

CONSUMER INVOLVEMENT: not stated.

Participants

DESCRIPTION: cardiac patients.

GEOGRAPHIC LOCATION: USA.

SETTING:discharged home from an acute care setting.

NUMBER OF PARTICIPANTS:

ELIGIBLE: 573.

RANDOMISED TO INTERVENTION: 130.

RANDOMISED TO CONTROL: 112 .

INCLUDED IN ANALYSIS INTERVENTION GROUP: 130

INCLUDED IN ANALYSIS CONTROL GROUP: 112.

AGE: RANGE OR MEAN (SD): 72 (12).

GENDER (\% MALE): 49 .

ETHNICITY: 25\% Spanish speaking.

PRINCIPAL HEALTH PROBLEM OR DIAGNOSIS: heart failure (49\% ischemic, 20\% hypertensive).

OTHER HEALTH PROBLEM/S: 41\% low comorbidity, 20\% high comorbidity (68\% hypertension, 35\% COPD, $42 \%$ diabetes, $28 \%$ renal disease).

TREATMENT RECEIVED/RECEIVING: medication (62\% digoxin, 54\% ACE-inhibitor, 86\% diuretic,...).

OTHER SOCIAL/DEMOGRAPHIC DETAILS: 56\% single.

Interventions

DETAILS OF INTERVENTION: Telephonic case management by a registered nurse was provided using a decision support software program; the software program uses automated tool for setting priorities for patient education, data collection and documentation; best practices are supported by the program. The intervention group was phoned within 5 days after discharge and thereafter at a frequency guided by the software and case manager judgment based on patient symptoms, knowledge and needs. Patients received an average of 17 calls at decreasing levels of intensity, length and frequency over the 6 month follow-up period; printed educational material was mailed every month to the patients.

DETAILS OF CONTROL: usual care, not standardized.

CO-INTERVENTION? unclear.

Telephone follow-up, initiated by a hospital-based health professional, for postdischarge problems in patients discharged from hospital 
Riegel 2002 (Continued)

DETAILS OF CO-INTERVENTIONS:

DELIVERY OF INTERVENTION

Frequency: 14.

First time at day 5 after discharge.

Period: 6 months.

PROVIDERS: nurse.

INTERVENTION QUALITY: large range.

FIDELITY/INTEGRITY: unclear.

\begin{tabular}{|c|c|c|}
\hline \multirow[t]{5}{*}{ Outcomes } & \multicolumn{2}{|c|}{ NUMBER OF OUTCOMES: 2} \\
\hline & \multicolumn{2}{|c|}{$\begin{array}{l}\text { OUTCOME / TOOL / TOOL VALIDATED / METHOD OF ASSESSMENT / TIME OF ASSESSMENT } \\
\text { A.Psycho-social health of patients (uncertainty, anxiety, informational needs, mood, coping, quality of } \\
\text { life, social activity, ..): }\end{array}$} \\
\hline & \multicolumn{2}{|c|}{ B.Physical health of patients (eg. functional status, self-care, self-efficacy, independence, ...): } \\
\hline & \multicolumn{2}{|c|}{ C.Other consumer oriented outcomes (eg. treatment adherence, knowledge, adverse events, ...): } \\
\hline & \multicolumn{2}{|c|}{$\begin{array}{l}\text { D. Health service delivery oriented outcomes (eg. hospital readmission, health services utilization, ...): } \\
\text { Readmission / self-developed / unclear / hospital record / } 3 \text { and } 6 \text { months. Costs / self-developed / no / } \\
\text { hospital record / } 3 \text { and } 6 \text { months. }\end{array}$} \\
\hline \multirow[t]{5}{*}{ Notes } & \multicolumn{2}{|c|}{$\begin{array}{l}\text {.CHANGES IN TRIAL PROTOCOL: Riegel } 2002 \text { (in journal Disease Management \& Health Outcomes) is } \\
\text { subanalysis for Spanish speaking patients. }\end{array}$} \\
\hline & \multicolumn{2}{|c|}{.CONTACT WITH AUTHOR } \\
\hline & \multicolumn{2}{|c|}{ ·POWER CALCULATION? yes. } \\
\hline & \multicolumn{2}{|c|}{$\cdot$ RECORD IF THE STUDY WAS TRANSLATED FROM A LANGUAGE OTHER THAN ENGLISH. } \\
\hline & \multicolumn{2}{|c|}{ ·RECORD IF THE STUDY WAS A DUPLICATE PUBLICATION. } \\
\hline \multicolumn{3}{|l|}{ Risk of bias } \\
\hline Bias & Authors' judgement & Support for judgement \\
\hline Allocation concealment? & Unclear risk & B - Unclear \\
\hline
\end{tabular}

Ritchie 2000

Methods

DETAILS OF STUDY

AIM OF INTERVENTION: to improve patient attendance at outpatients clinic after ED visit.

AIM OF STUDY: to determine whether the intervention of a telephone call within 3 days of ED attendance would improve the proportion of patients making recommended appointments and the proportion of patients attending scheduled appointments.

STUDY DESIGN: RCT.

METHODS OF RECRUITMENT OF PARTICIPANTS: consecutive patients who were advised by ED doctors to make outpatient appointments were identified for inclusion using the ED computer system over a 4 week period.

INCLUSION CRITERIA FOR PARTICIPATION IN STUDY:

Telephone follow-up, initiated by a hospital-based health professional, for postdischarge problems in patients discharged from hospital 
Ritchie 2000 (Continued)

EXCLUSION CRITERIA FOR PARTICIPATION IN STUDY: do not speak english/ confused or demented with no carer/had no telephone/ age under 16 years/haven been included previously in the study/are referred to a private specialist or another hospital.

INFORMED CONSENT OBTAINED? no.

ETHICAL APPROVAL? yes.

FUNDING: unclear

\section{ASSESSMENT OF STUDY QUALITY}

ALLOCATION CONCEALMENT: inadequate.

EPOC- QUALITY CRITERIA 2002: B. moderate risk of bias.

METHOD OF GENERATING RANDOMISATION SCHEDULE: restricted randomisation in blocks of ten was performed using a table of random numbers from a standard statistical text.

METHOD OF CONCEALMENT OF ALLOCATION: not specified.

BLINDING:

-PARTICIPANTS: no.

-PROVIDER/S: no.

-OUTCOME ASSESSOR/S: no.

INTENTION TO TREAT ANALYSIS: yes.

BASELINE COMPARABILITY OF INTERVENTION AND CONTROL GROUPS: yes.

STATISTICAL METHODS AND THEIR APPROPRIATENESS: chi-square/ logistic regression.

CONSUMER INVOLVEMENT: not stated.

Participants

DESCRIPTION: ED patients.

GEOGRAPHIC LOCATION: Australia.

SETTING: discharged home from an acute care setting.

NUMBER OF PARTICIPANTS:

ELIGIBLE: 400.

RANDOMISED TO INTERVENTION: 200

RANDOMISED TO CONTROL: 180.

INCLUDED IN ANALYSIS INTERVENTION GROUP: 164.

INCLUDED IN ANALYSIS CONTROL GROUP: 180.

AGE: RANGE OR MEAN (SD): 16-65+ .

GENDER (\% MALE): 64.

ETHNICITY: not stated.

PRINCIPAL HEALTH PROBLEM OR DIAGNOSIS: various.

OTHER HEALTH PROBLEM/S:

TREATMENT RECEIVED/RECEIVING: unclear.

OTHER SOCIAL/DEMOGRAPHIC DETAILS:

Interventions

DETAILS OF INTERVENTION: patients in the intervention group were phoned by one of the authors (MD) or a research nurse 1-3 days after ED attendance. A general enquiry was made about their health and the importance of medical follow-up was explained in general terms; if the patient had already made an appointment, they were reminded about that appointment; for those who had not yet scheduled an

Telephone follow-up, initiated by a hospital-based health professional, for postdischarge problems in patients discharged from hospital

Copyright (c) 2010 The Cochrane Collaboration. Published by John Wiley \& Sons, Ltd. 
Ritchie 2000 (Continued)

appointment, they were reminded that they had been advised to do so, and an offer was made to book one for them at that time.

DETAILS OF CONTROL: usual care.

CO-INTERVENTION? no.

DETAILS OF CO-INTERVENTIONS:

DELIVERY OF INTERVENTION

Frequency: 1 .

First time at day 1-3 after discharge.

Period:

PROVIDERS: doctor/nurse.

INTERVENTION QUALITY: unclear,

FIDELITY/INTEGRITY: unclear.

Outcomes

NUMBER OF OUTCOMES: 1.

OUTCOME / TOOL / TOOL VALIDATED / METHOD OF ASSESSMENT / TIME OF ASSESSMENT

A.Psycho-social health of patients (uncertainty, anxiety, informational needs, mood, coping, quality of life, social activity, ..):

B.Physical health of patients (eg. functional status, self-care, self-efficacy, independence, ...):

C.Other consumer oriented outcomes (eg. treatment adherence, knowledge, adverse events, ...): Compliance / self-developed / no / hospital record / within 3 months.

D. Health service delivery oriented outcomes (eg. hospital readmission, health services utilization, ..):

$\begin{array}{ll}\text { Notes } & \cdot \text { CHANGES IN TRIAL PROTOCOL } \\ & \cdot \text { CONTACT WITH AUTHOR } \\ & \cdot \text { POWER CALCULATION? Yes. } \\ & \cdot \text { RECORD IF THE STUDY WAS TRANSLATED FROM A LANGUAGE OTHER THAN ENGLISH. } \\ & \cdot \text { RECORD IF THE STUDY WAS A DUPLICATE PUBLICATION. }\end{array}$

\section{Risk of bias}

\begin{tabular}{lll}
\hline Bias & Authors' judgement & Support for judgement \\
\hline Allocation concealment? & High risk & C - Inadequate \\
\hline
\end{tabular}

\section{Roebuck 1999}

DETAILS OF STUDY
AIM OF INTERVENTION: to reduce anxiety and depression.
AIM OF STUDY: the study tests the hypothesis that telephone follow-up from the ward will reduce pa-
tients' anxiety and depression levels in the early post-discharge period.
STUDY DESIGN: CCT.
METHODS OF RECRUITMENT OF PARTICIPANTS: patients admitted during a 21-day consecutive period
were enrolled to a defined group. After completion of this period, no patients were enrolled for 7 days

Telephone follow-up, initiated by a hospital-based health professional, for postdischarge problems in patients discharged from hospital $\mathbf{8 7}$ to home (Review)

Copyright (c) 2010 The Cochrane Collaboration. Published by John Wiley \& Sons, Ltd. 
Roebuck 1999 (Continued)

to prevent patient overlap if their discharge was delayed. The process was then repeated with the patients being enrolled to the alternative group. Patients were asked for consent and enrolled into the study on the day they were given a planned discharge date.

INCLUSION CRITERIA FOR PARTICIPATION IN STUDY: have undergone elective coronary artery bypasS grafting or valve replacement.

EXCLUSION CRITERIA FOR PARTICIPATION IN STUDY: unable to give consent/ could not communicate in English/ history of mental illness/ have undergone emergency cardiac surgery.

INFORMED CONSENT OBTAINED? yes.

ETHICAL APPROVAL? yes.

FUNDING: yes.

ASSESSMENT OF STUDY QUALITY

ALLOCATION CONCEALMENT: inadequate.

EPOC- QUALITY CRITERIA 2002: C.high risk of bias.

METHOD OF GENERATING RANDOMISATION SCHEDULE: A convenience sampling model utilizing alternative block selection was used.

METHOD OF CONCEALMENT OF ALLOCATION: not specified.

BLINDING:

-PARTICIPANTS: no.

-PROVIDER/S: no.

-OUTCOME ASSESSOR/S: no.

INTENTION TO TREAT ANALYSIS: not stated.

BASELINE COMPARABILITY OF INTERVENTION AND CONTROL GROUPS: yes.

STATISTICAL METHODS AND THEIR APPROPRIATENESS: Mann-Whitney U test.

CONSUMER INVOLVEMENT: not stated.

Participants

DESCRIPTION: cardiac patients / surgery patients.

GEOGRAPHIC LOCATION: UK.

SETTING: discharged home from an acute care setting.

NUMBER OF PARTICIPANTS:

ELIGIBLE: 78.

RANDOMISED TO INTERVENTION: 45.

RANDOMISED TO CONTROL: 31.

INCLUDED IN ANALYSIS INTERVENTION GROUP: 42

INCLUDED IN ANALYSIS CONTROL GROUP: 31.

AGE: RANGE OR MEAN (SD): unclear.

GENDER (\% MALE): unclear.

ETHNICITY: unclear.

PRINCIPAL HEALTH PROBLEM OR DIAGNOSIS: coronary heart and/or valve disease.

OTHER HEALTH PROBLEM/S: not specified.

TREATMENT RECEIVED/RECEIVING: CABG-surgery or valve replacement. 
Roebuck 1999 (Continued)

OTHER SOCIAL/DEMOGRAPHIC DETAILS: not given.

Interventions

DETAILS OF INTERVENTION: patients in the intervention group received standard discharge advice/information plus 2 additional follow-up calls from a nurse. Contact was made at 7 and 21 days after discharge. On contact, the callers introduced themselves and patient identity was confirmed. Patients were invited to discuss how they had been since their last contact with the ward and encouraged to raise any concerns or difficulties they had experienced since that time.

DETAILS OF CONTROL: standard advice included the patient being offered a selection of information leaflets, a personal exercise plan and an individual discussion with their named nurse over concerns and discharge medications.

CO-INTERVENTION? no.

DETAILS OF CO-INTERVENTIONS:

DELIVERY OF INTERVENTION

Frequency: 2.

First time at day 7 after discharge.

Period: 3 weeks.

PROVIDERS: nurse.

INTERVENTION QUALITY: unclear.

FIDELITY/INTEGRITY: unclear.

Outcomes NUMBER OF OUTCOMES: 2

OUTCOME / TOOL / TOOL VALIDATED / METHOD OF ASSESSMENT / TIME OF ASSESSMENT

A.Psycho-social health of patients (uncertainty, anxiety, informational needs, mood, coping, quality of life, social activity, ...): Anxiety / Hospital anxiety and depression scale (Zigmond, 1983) / yes / postal questionnaire / 5 weeks after discharge. Depression / Hospital anxiety and depression scale (Zigmond, 1983) / yes / postal questionnaire / 5 weeks after discharge.

B.Physical health of patients (eg. functional status, self-care, self-efficacy, independence, ...):

C.Other consumer oriented outcomes (eg. treatment adherence, knowledge, adverse events, ...):

D. Health service delivery oriented outcomes (eg. hospital readmission, health services utilization, ..):

\section{Notes}

-CHANGES IN TRIAL PROTOCOL

·CONTACT WITH AUTHOR

-POWER CALCULATION?

-RECORD IF THE STUDY WAS TRANSLATED FROM A LANGUAGE OTHER THAN ENGLISH.

-RECORD IF THE STUDY WAS A DUPLICATE PUBLICATION.

\section{Risk of bias}

\begin{tabular}{lll}
\hline Bias & Authors' judgement & Support for judgement \\
\hline Allocation concealment? & High risk & C - Inadequate \\
\hline
\end{tabular}

Samarel 2002

Methods DETAILS OF STUDY

Telephone follow-up, initiated by a hospital-based health professional, for postdischarge problems in patients discharged from hospital 
AIM OF INTERVENTION: to reduce worry, mood disturbance and enhance well-being and quality of relationship.

AIM OF STUDY: the purpose of this study was to examine the effects of a 13-month, 3 phase intervention, comparing an experimental group receiving combined individual telephone and in-person group social support and education treatment with a control group receiving telephone only individual support and education treatment and a control group receiving one time mailed educational information treatment on cancer-related worry, well-being, mood disturbance, loneliness and the quality of relationship with a significant other among women newly diagnosed with early stage breast cancer.

\title{
STUDY DESIGN: CCT.
}

METHODS OF RECRUITMENT OF PARTICIPANTS: Women were recruited through letters distributed at physician's offices, hospitals and the American Cancer Society Reach to Recover program. Because letters were distributed by personnel at each site, the number of women reached is not known.

INCLUSION CRITERIA FOR PARTICIPATION IN STUDY: English speaking/ had surgery for nonmetastatic breast cancer within 4 weeks prior to beginning their participation in the study, had no previous cancer diagnosis, no other major medical problems.

EXCLUSION CRITERIA FOR PARTICIPATION IN STUDY: not specified.

INFORMED CONSENT OBTAINED? yes.

ETHICAL APPROVAL? unclear.

FUNDING: yes.

ASSESSMENT OF STUDY QUALITY

ALLOCATION CONCEALMENT: adequate.

EPOC- QUALITY CRITERIA 2002: C.high risk of bias.

METHOD OF GENERATING RANDOMISATION SCHEDULE: Using a permuted block design for randomization, when successive cohorts of 4-8 women had been recruited, the entire cohort was randomly assigned to one of the three treatment groups using the sealed opaque envelope technique. When the next cohort was recruited, that cohort was assigned to one of the two remaining study treatment groups, using the two remaining sealed opaque envelopes. The next cohort was assigned to the remaining study treatment group, after which the process started again. Random assignment was repeated in this manner until the full sample was recruited and assigned.

METHOD OF CONCEALMENT OF ALLOCATION: sealed opaque envelopes.

BLINDING:

-PARTICIPANTS: no.

-PROVIDER/S: no.

-OUTCOME ASSESSOR/S: no.

INTENTION TO TREAT ANALYSIS: not stated.

BASELINE COMPARABILITY OF INTERVENTION AND CONTROL GROUPS: no.

STATISTICAL METHODS AND THEIR APPROPRIATENESS: manova/ anova/kruskall-wallis/ mann-whitney U.

CONSUMER INVOLVEMENT: not stated.

\footnotetext{
Participants
}

\author{
DESCRIPTION: oncology patients / surgery patients. \\ GEOGRAPHIC LOCATION: USA.
}

SETTING: discharged home from an acute care setting.

NUMBER OF PARTICIPANTS:

Telephone follow-up, initiated by a hospital-based health professional, for postdischarge problems in patients discharged from hospital 
Samarel 2002 (Continued)

\author{
ELIGIBLE: 183. \\ RANDOMISED TO INTERVENTION: 68 \\ RANDOMISED TO CONTROL: 34 - 60. \\ INCLUDED IN ANALYSIS INTERVENTION GROUP: 48. \\ INCLUDED IN ANALYSIS CONTROL GROUP: 34 - 60.
}

AGE: RANGE OR MEAN (SD): 54 (10.8).

GENDER (\% MALE): 0.

ETHNICITY: 89\% white.

PRINCIPAL HEALTH PROBLEM OR DIAGNOSIS: breast cancer.

OTHER HEALTH PROBLEM/S: not specified.

TREATMENT RECEIVED/RECEIVING: lumpectomy (42\%), mastectomy (57), chemotherapy (44\%), radiation therapy (26\%).

OTHER SOCIAL/DEMOGRAPHIC DETAILS: $61 \%$ married, 43\% high school.

Interventions

DETAILS OF INTERVENTION: 3 groups: telephone only, telephone and in-person group social support, and group 3 one time education by mailing. Women in (for this review experimental group) experienced a less intense form of the focal stimulus by receiving social support and education only by telephone in all 3 phases of the study (see control group 1). the intervention was based on Roy's adaptation model. The individual telephone social support and education were provided by either oncology nurse clinicians or social workers, who were trained by the investigators. Specially developed guides were used during the intervention. Logs of the phone contacts were made, that were periodically reviewed.

DETAILS OF CONTROL: This group received the most intense intervention in the form of the focal stimulus of social support and education by receiving combined individual telephone and in-person group social support and education, which was provided in 3 phases over 13 months. The treatment was designed to provide more intense support during the times of peak need. More specifically weekly phone contacts in first 3 months, weekly in-person social support in next two months and twice-monthly phone contacts in next 8 months. Control group 2 received usual care in first 3 months, and one-time mailing in month 4.

\title{
CO-INTERVENTION? no.
}

\section{DETAILS OF CO-INTERVENTIONS:}

\section{DELIVERY OF INTERVENTION}

Frequency: 32.

First time at day between week 2 and 4 after discharge.

Period: 13 months.

PROVIDERS: nurse/social worker.

INTERVENTION QUALITY: unclear.

FIDELITY/INTEGRITY: good.

Outcomes NUMBER OF OUTCOMES: 7

OUTCOME / TOOL / TOOL VALIDATED / METHOD OF ASSESSMENT / TIME OF ASSESSMENT

A.Psycho-social health of patients (uncertainty, anxiety, informational needs, mood, coping, quality of life, social activity, ..): Satisfaction / patient satisfaction questionnaire (Shortell 2000) / yes / telephone interview / 1 and 5 weeks after discharge. Mental status / SF-36 (Ware, 1992) / yes / telephone interview / 1 and 5 weeks after discharge. Social functioning / SF-36 (Ware, 1992) / yes / telephone interview / 1 and 5 weeks after discharge.

B.Physical health of patients (eg. functional status, self-care, self-efficacy, independence, ..): Physical functioning / SF-36 (Ware, 1992) / yes / telephone interview / 1 and 5 weeks after discharge. Symptom

Telephone follow-up, initiated by a hospital-based health professional, for postdischarge problems in patients discharged from hospital 
distress / Memorial symptom assessment scale (Portenoy 1994) / yes / telephone interview / 1 and 5 weeks after discharge.

C.Other consumer oriented outcomes (eg. treatment adherence, knowledge, adverse events, ..):

D. Health service delivery oriented outcomes (eg. hospital readmission, health services utilization, ..): Readmission / self-developed / no / telephone interview / 1 and 5 weeks after discharge. ED-visits / selfdeveloped / no / telephone interview / 1 and 5 weeks after discharge.

Notes $\quad$ CHANGES IN TRIAL PROTOCOL

NOTE: since intervention group and control group 1 receive the same intervention during first 3 months and they only start differing after this point, these groups can be combined for this review into 1 intervention group and compared to control group 2 (usual care).

-CONTACT WITH AUTHOR

-POWER CALCULATION? yes.

·RECORD IF THE STUDY WAS TRANSLATED FROM A LANGUAGE OTHER THAN ENGLISH.

-RECORD IF THE STUDY WAS A DUPLICATE PUBLICATION.

\section{Risk of bias}

\begin{tabular}{lll}
\hline Bias & Authors' judgement & Support for judgement \\
\hline Allocation concealment? & Low risk & A - Adequate \\
\hline
\end{tabular}

Shesser 1986

AIM OF INTERVENTION: to increase patient satisfaction and patient compliance.

AIM OF STUDY: to describe and quantify the benefit that can be derived from an organized system of follow-up telephone calls.

\section{STUDY DESIGN: CCT.}

METHODS OF RECRUITMENT OF PARTICIPANTS: During a 10 day period, all patients charts were reviewed and each patient who was discharged home from the ED with one of nine diagnosis was included in the study; included patients then were stratified by disease category and were randomized into two groups.

INCLUSION CRITERIA FOR PARTICIPATION IN STUDY: Discharged home/ diagnosis of undiagnosed chest pain, undiagnosed abdominal pain, acute infection, vaginal haemorrhage, syncope, acute cervical/lumbar pain, asthma/bronchospasm, allergic reaction, headache.

EXCLUSION CRITERIA FOR PARTICIPATION IN STUDY: not specified.

INFORMED CONSENT OBTAINED? no.

ETHICAL APPROVAL? unclear.

FUNDING: unclear.

\section{ASSESSMENT OF STUDY QUALITY}

ALLOCATION CONCEALMENT: unclear.

EPOC- QUALITY CRITERIA 2002: C.high risk of bias. 
METHOD OF GENERATING RANDOMISATION SCHEDULE: not specified.

METHOD OF CONCEALMENT OF ALLOCATION: not specified.

BLINDING:

PARTICIPANTS: no.

-PROVIDER/S: no.

.OUTCOME ASSESSOR/S: no.

INTENTION TO TREAT ANALYSIS: not stated.

BASELINE COMPARABILITY OF INTERVENTION AND CONTROL GROUPS: yes.

STATISTICAL METHODS AND THEIR APPROPRIATENESS: chi-square.

CONSUMER INVOLVEMENT: not stated.

Participants

DESCRIPTION: ED patients.

GEOGRAPHIC LOCATION: USA.

SETTING: discharged home from an acute care setting.

NUMBER OF PARTICIPANTS:

ELIGIBLE: 559.

RANDOMISED TO INTERVENTION: 297.

RANDOMISED TO CONTROL: 94.

INCLUDED IN ANALYSIS INTERVENTION GROUP: 83.

INCLUDED IN ANALYSIS CONTROL GROUP: 94.

AGE: RANGE OR MEAN (SD): 36 (15).

GENDER (\% MALE): 47.

ETHNICITY: unclear.

PRINCIPAL HEALTH PROBLEM OR DIAGNOSIS: acute infection (30\%), abdominal pain (20\%), acute lumbar pain (14\%), chest pain (12\%), other.

OTHER HEALTH PROBLEM/S: not specified.

TREATMENT RECEIVED/RECEIVING: unclear.

OTHER SOCIAL/DEMOGRAPHIC DETAILS:

Interventions

DETAILS OF INTERVENTION: Within 48 to 72 hours after the ED-visit, attempts were made to call the patients. Calls were made by members of the ED clinical nursing staff. The caller had a copy of the patient's ED chart, and interviewed the patient by following a written scenario that was designed to determine the progression of the patient's symptoms, whether the patient had already sought additional medical consultation, whether the patient eventually would seek follow-up with the provider recommended by the ED physician and whether the ED instructions for aftercare were clear.

DETAILS OF CONTROL: usual care.

CO-INTERVENTION? no.

DETAILS OF CO-INTERVENTIONS:

DELIVERY OF INTERVENTION

Frequency: 1.

First time at day 2-3 after discharge.

Period:

PROVIDERS: nurse. 
INTERVENTION QUALITY: unclear.

FIDELITY/INTEGRITY: unclear.

Outcomes NUMBER OF OUTCOMES: 7

OUTCOME / TOOL / TOOL VALIDATED / METHOD OF ASSESSMENT / TIME OF ASSESSMENT

A.Psycho-social health of patients (uncertainty, anxiety, informational needs, mood, coping, quality of life, social activity, ...): Satisfaction / patient satisfaction questionnaire (Shortell 2000) / yes / telephone interview / 1 and 5 weeks after discharge. Mental status / SF-36 (Ware, 1992) / yes / telephone interview / 1 and 5 weeks after discharge. Social functioning / SF-36 (Ware, 1992) / yes / telephone interview / 1 and 5 weeks after discharge.

B.Physical health of patients (eg. functional status, self-care, self-efficacy, independence, ..): Physical functioning / SF-36 (Ware, 1992) / yes / telephone interview / 1 and 5 weeks after discharge. Symptom distress / Memorial symptom assessment scale (Portenoy 1994) / yes / telephone interview / 1 and 5 weeks after discharge.

C.Other consumer oriented outcomes (eg. treatment adherence, knowledge, adverse events, ..):

D. Health service delivery oriented outcomes (eg. hospital readmission, health services utilization, ..): Readmission / self-developed / no / telephone interview / 1 and 5 weeks after discharge. ED-visits / selfdeveloped / no / telephone interview / 1 and 5 weeks after discharge.

\section{Notes}

- CHANGES IN TRIAL PROTOCOL

-CONTACT WITH AUTHOR

-POWER CALCULATION?

-RECORD IF THE STUDY WAS TRANSLATED FROM A LANGUAGE OTHER THAN ENGLISH.

-RECORD IF THE STUDY WAS A DUPLICATE PUBLICATION.

\section{Risk of bias}

\begin{tabular}{lll}
\hline Bias & Authors' judgement & Support for judgement \\
\hline Allocation concealment? & Unclear risk & B - Unclear \\
\hline
\end{tabular}

\section{Touyz 1998}

\section{Methods}

DETAILS OF STUDY

AIM OF INTERVENTION: to reduce pain and improve pain management.

AIM OF STUDY: to determine whether telephone consultation influenced patients' perception of and reaction to pain after periodontal surgery.

\section{STUDY DESIGN: CCT.}

METHODS OF RECRUITMENT OF PARTICIPANTS: during a 4 year period patients who presented to the division of periodontology of a general hospital and who fulfilled inclusion criteria were admitted to the study.

INCLUSION CRITERIA FOR PARTICIPATION IN STUDY: moderate to severe peridontal disease (class 3 and 4), diagnosed as adult cause-related peridontitis, root planning and subsequent periodontal surgical pocket reduction or prescribed elective preprosthetic periodontal surgery, systemic health,age 30 to 70 years, no history of mental disease, no medication for at least 1 month prior to the procedure.

EXCLUSION CRITERIA FOR PARTICIPATION IN STUDY: not specified.

Telephone follow-up, initiated by a hospital-based health professional, for postdischarge problems in patients discharged from hospital 
Touyz 1998 (Continued)

INFORMED CONSENT OBTAINED? no.

ETHICAL APPROVAL? unclear.

FUNDING: unclear.

ASSESSMENT OF STUDY QUALITY

ALLOCATION CONCEALMENT: inadequate.

EPOC- QUALITY CRITERIA 2002: C. high risk of bias.

METHOD OF GENERATING RANDOMISATION SCHEDULE: not done.

METHOD OF CONCEALMENT OF ALLOCATION: not done.

BLINDING:

-PARTICIPANTS: no.

-PROVIDER/S: no.

-OUTCOME ASSESSOR/S: no.

INTENTION TO TREAT ANALYSIS: not stated.

BASELINE COMPARABILITY OF INTERVENTION AND CONTROL GROUPS: not stated.

STATISTICAL METHODS AND THEIR APPROPRIATENESS: student t-test.

CONSUMER INVOLVEMENT: not stated.

Participants

DESCRIPTION: surgery patients.

GEOGRAPHIC LOCATION: Canada.

SETTING: discharged home from an acute care setting.

NUMBER OF PARTTICIPANTS:

ELIGIBLE: 152.

RANDOMISED TO INTERVENTION: 59.

RANDOMISED TO CONTROL: 59.

INCLUDED IN ANALYSIS INTERVENTION GROUP: 59.

INCLUDED IN ANALYSIS CONTROL GROUP: 59.

AGE: RANGE OR MEAN (SD): 50 (2).

GENDER (\% MALE): 46.

ETHNICITY: unclear.

PRINCIPAL HEALTH PROBLEM OR DIAGNOSIS: periodontitis.

OTHER HEALTH PROBLEM/S: none.

TREATMENT RECEIVED/RECEIVING: periodontal surgery.

OTHER SOCIAL/DEMOGRAPHIC DETAILS:

Interventions

DETAILS OF INTERVENTION: the intervention group patients were called no later than 24 hours after the procedure. The telephone interviewer was either the assisting student or the supervisor dentist, who then systematically inquired about 10 points (well-being of patient, the return to normal and loss of analgesia, jaw swelling, wound bleeding, whether the wound was painful, acquisition and use of mouthwash and analgesics, the need for a soft balanced diet, necessity of sustained oral hygiene, confirmation of the next week's appointment and reassurance about the reaction and pain). The interviewer was instructed to be sympathetic, to reassure patients that whatever reaction they were having was within the range of expected normal limits and to be positive about a successful outcome.

Telephone follow-up, initiated by a hospital-based health professional, for postdischarge problems in patients discharged from hospital 
Touyz 1998 (Continued)

DETAILS OF CONTROL: usual care.

CO-INTERVENTION? no.

DETAILS OF CO-INTERVENTIONS:

DELIVERY OF INTERVENTION

Frequency: 1 .

First time at day 1 after discharge.

Period:

PROVIDERS: dentist.

INTERVENTION QUALITY: unclear.

FIDELITY/INTEGRITY: unclear.

Outcomes NUMBER OF OUTCOMES: 3

OUTCOME / TOOL / TOOL VALIDATED / METHOD OF ASSESSMENT / TIME OF ASSESSMENT

A.Psycho-social health of patients (uncertainty, anxiety, informational needs, mood, coping, quality of life, social activity, ...):

B.Physical health of patients (eg. functional status, self-care, self-efficacy, independence, ..): Selfcare deficits / self-developed / no / telephone interview / 6 weeks after discharge. Blood glucose level / Hba1c-level / unclear / blood sample / 3 months after discharge.

C.Other consumer oriented outcomes (eg. treatment adherence, knowledge, adverse events, ..): Knowledge / Diabetes knowledge scale (Dunn,1984) / yes / telephone interview / 6 weeks after discharge.

D. Health service delivery oriented outcomes (eg. hospital readmission, health services utilization, ...):

Notes $\quad$ CHANGES IN TRIAL PROTOCOL

-CONTACT WITH AUTHOR

·POWER CALCULATION?

·RECORD IF THE STUDY WAS TRANSLATED FROM A LANGUAGE OTHER THAN ENGLISH.

-RECORD IF THE STUDY WAS A DUPLICATE PUBLICATION.

\section{Risk of bias}

\begin{tabular}{lll}
\hline Bias & Authors' judgement & Support for judgement \\
\hline Allocation concealment? & High risk & C - Inadequate \\
\hline
\end{tabular}

Methods DETAILS OF STUDY

AIM OF INTERVENTION: to improve cardiac surgery recovery following hospital discharge, as reflected in improved health related quality of life, decreased symptom distress, improved satisfaction with discharge and follow-up care and decreased unplanned contacts with the hospital.

AIM OF STUDY: The purpose of the study was to determine if the provision of Advanced Practice Nurse (APN) support, delivered via the telephone, improved cardiac surgery recovery following hospital discharge, as reflected in improved health related quality of life, decreased symptom distress, improved satisfaction with discharge and follow-up care and decreased unplanned contacts with the hospital. 


\section{STUDY DESIGN: RCT.}

METHODS OF RECRUITMENT OF PARTICIPANTS: participants were recruited from the inpatient cardiac surgery unit. Prior to discharge, eligible participants were identified by a research assistant who obtained consent and baseline data. The research assistant informed the APN of consenting patients' discharge. recruited patients were randomized after discharge.

INCLUSION CRITERIA FOR PARTICIPATION IN STUDY: a) undergone first elective or emergent cardiac surgery, b) no unexpected cardiac complications that necessitated an unexpected stay in ICU, c) oriented to time, place and person, d) no history of acute or chronic psychiatric problems, e) able to read, write and speak English, f) capable of responding over the telephone.

EXCLUSION CRITERIA FOR PARTICIPATION IN STUDY

INFORMED CONSENT OBTAINED? yes.

ETHICAL APPROVAL? yes.

FUNDING: yes.

ASSESSMENT OF STUDY QUALITY

ALLOCATION CONCEALMENT: unclear.

EPOC- QUALITY CRITERIA 2002: B.moderate risk of bias.

METHOD OF GENERATING RANDOMISATION SCHEDULE: random numbers were generated by a statistical consultant through a computer based randomization schedule and forwarded to the APN who assigned patients.

METHOD OF CONCEALMENT OF ALLOCATION: unclear.

BLINDING:

-PARTICIPANTS: no.

-PROVIDER/S: no.

-OUTCOME ASSESSOR/S: yes.

INTENTION TO TREAT ANALYSIS: yes.

BASELINE COMPARABILITY OF INTERVENTION AND CONTROL GROUPS: yes.

STATISTICAL METHODS AND THEIR APPROPRIATENESS: t-test/chi-square/Mann-Whitney U test.

CONSUMER INVOLVEMENT: not stated.

Participants

DESCRIPTION: cardiac patients / surgery patients.

GEOGRAPHIC LOCATION: Canada.

SETTING: discharged home from an acute care setting.

NUMBER OF PARTICIPANTS:

ELIGIBLE: unclear.

RANDOMISED TO INTERVENTION: 102.

RANDOMISED TO CONTROL: 92.

INCLUDED IN ANALYSIS INTERVENTION GROUP: 94.

INCLUDED IN ANALYSIS CONTROL GROUP: 92.

AGE: RANGE OR MEAN (SD): 64.

GENDER (\% MALE): 76.

ETHNICITY: unclear.

PRINCIPAL HEALTH PROBLEM OR DIAGNOSIS: coronary artery disease.

Telephone follow-up, initiated by a hospital-based health professional, for postdischarge problems in patients discharged from hospital

Copyright $\odot 2010$ The Cochrane Collaboration. Published by John Wiley \& Sons, Ltd. 
OTHER HEALTH PROBLEM/S: COPD in 20\%; peripheral vascular disease in $10 \%$, cerebrovascular disease in 145 , renal failure in $3.5 \%$.

TREATMENT RECEIVED/RECEIVING: CABG.

OTHER SOCIAL/DEMOGRAPHIC DETAILS: 16\% single.

Interventions

DETAILS OF INTERVENTION: The intervention group received, in addition to the usual care, active and ongoing follow-up via nurse-initiated telephone calls from the APN in cardiac surgery at 3 and 5 days following discharge and then weekly for 4 more weeks. During the calls, the APN continued with the plan of care established in the hospital, provided ongoing information and assessment, assisted with self-management of common symptoms and facilitated referrals to appropriate healthcare resources. Telephone sessions were individually tailored in response to patient's symptoms, concerns and recovery. All sessions, however, were standardized sufficiently to include evaluation of physical and psychological states and incorporated mutual goal setting for the management of symptoms in the recovery period. The initial TFU were approximately 20 to 30 minutes.

DETAILS OF CONTROL: usual care: this included preoperative and discharge preparation by the APN, provision of an education booklet and home care follow-up if necessary. Patients were provided the contact information for the APN and instruction to call with questions or concerns.

CO-INTERVENTION? no.

DETAILS OF CO-INTERVENTIONS:

DELIVERY OF INTERVENTION

Frequency: 6.

First time at day 3 after discharge.

Period: 5 weeks.

PROVIDERS: nurse.

INTERVENTION QUALITY: good.

FIDELITY/INTEGRITY: good.

OUTCOME / TOOL / TOOL VALIDATED / METHOD OF ASSESSMENT / TIME OF ASSESSMENT A.Psycho-social health of patients (uncertainty, anxiety, informational needs, mood, coping, quality of life, social activity, ...): Satisfaction / patient satisfaction questionnaire (Shortell 2000) / yes / telephone interview / 1 and 5 weeks after discharge. Mental status / SF-36 (Ware, 1992) / yes / telephone interview / 1 and 5 weeks after discharge. Social functioning / SF-36 (Ware, 1992) / yes / telephone interview / 1 and 5 weeks after discharge.

B.Physical health of patients (eg. functional status, self-care, self-efficacy, independence, ..): Physical functioning / SF-36 (Ware, 1992) / yes / telephone interview / 1 and 5 weeks after discharge. Symptom distress / Memorial symptom assessment scale (Portenoy 1994) / yes / telephone interview / 1 and 5 weeks after discharge.

C.Other consumer oriented outcomes (eg. treatment adherence, knowledge, adverse events, ..):

D. Health service delivery oriented outcomes (eg. hospital readmission, health services utilization, ..): Readmission / self-developed / no / telephone interview / 1 and 5 weeks after discharge. ED-visits / selfdeveloped / no / telephone interview / 1 and 5 weeks after discharge.

Notes

- CHANGES IN TRIAL PROTOCOL

-CONTACT WITH AUTHOR

-POWER CALCULATION? yes.

·RECORD IF THE STUDY WAS TRANSLATED FROM A LANGUAGE OTHER THAN ENGLISH. 
Tranmer 2004 (Continued)

·RECORD IF THE STUDY WAS A DUPLICATE PUBLICATION.

\section{Risk of bias}

\begin{tabular}{lll}
\hline Bias & Authors' judgement & Support for judgement \\
\hline Allocation concealment? & Unclear risk & B - Unclear \\
\hline
\end{tabular}

Tu 1993

Methods

DETAILS OF STUDY

AIM OF INTERVENTION: to increase self-care knowledge, improve metabolic control and reduce selfcare behavioral deficits.

AIM OF STUDY: to determine the effect of telephone follow-up on diabetes self-care knowledge, blood glucose levels, and changes in self-care behaviors of the elderly subjects.

\section{STUDY DESIGN: CCT.}

METHODS OF RECRUITMENT OF PARTICIPANTS: a convenience sample was recruited from inpatients of a diabetic hospital.

INCLUSION CRITERIA FOR PARTICIPATION IN STUDY: 60 years and older/ diabetes mellitus 2/ successfully completed an inpatient diabetes education program during their hospitalisation/ intact cognitive functioning/ able to perform self-care activities independently/ are being followed by primary physician in the diabetes clinic.

EXCLUSION CRITERIA FOR PARTICIPATION IN STUDY: complex medical conditions (heart failure, endstage renal disease, advanced cancer, major surgery,..).

INFORMED CONSENT OBTAINED? yes.

ETHICAL APPROVAL? yes.

FUNDING: yes.

ASSESSMENT OF STUDY QUALITY

ALLOCATION CONCEALMENT: unclear.

EPOC- QUALITY CRITERIA 2002: C.high risk of bias.

METHOD OF GENERATING RANDOMISATION SCHEDULE: not specified.

METHOD OF CONCEALMENT OF ALLOCATION: not specified.

BLINDING:

-PARTICIPANTS: no.

-PROVIDER/S: no.

.OUTCOME ASSESSOR/S: no.

INTENTION TO TREAT ANALYSIS: not stated.

BASELINE COMPARABILITY OF INTERVENTION AND CONTROL GROUPS: not stated.

STATISTICAL METHODS AND THEIR APPROPRIATENESS: t-test/chi-square.

CONSUMER INVOLVEMENT: not stated. 
Tu 1993 (Continued)

GEOGRAPHIC LOCATION: USA.

SETTING: discharged home from an acute care setting.

NUMBER OF PARTICIPANTS:

ELIGIBLE: 36.

RANDOMISED TO INTERVENTION: 16.

RANDOMISED TO CONTROL: 12

INCLUDED IN ANALYSIS INTERVENTION GROUP: 15.

INCLUDED IN ANALYSIS CONTROL GROUP: 12.

AGE: RANGE OR MEAN (SD): 65 (6.5).

GENDER (\% MALE): 33.

ETHNICITY: 52\% white.

PRINCIPAL HEALTH PROBLEM OR DIAGNOSIS: diabetes 2.

OTHER HEALTH PROBLEM/S: not specified.

TREATMENT RECEIVED/RECEIVING: education program.

OTHER SOCIAL/DEMOGRAPHIC DETAILS: 63\% had 12 years of education.

DETAILS OF INTERVENTION: subjects in the experimental group were contacted by either the primary investigator or a trained research assistant (both nurses) within 24 to 48 hours after discharge from hospital. The telephone calls were repeated at weekly intervals for 3 weeks, thus a total of 4 calls were made. Each call consisted of assessing the diabetic client's self care knowledge and practice of self-care activities or behaviours; supplemental instructions were provided when indicated.

DETAILS OF CONTROL: usual care.

CO-INTERVENTION? no.

DETAILS OF CO-INTERVENTIONS:

DELIVERY OF INTERVENTION

Frequency: 4.

First time at day 1-2 after discharge.

Period: 4.

PROVIDERS: nurse.

INTERVENTION QUALITY: unclear.

FIDELITY/INTEGRITY: good.

Outcomes NUMBER OF OUTCOMES: 3

OUTCOME / TOOL / TOOL VALIDATED / METHOD OF ASSESSMENT / TIME OF ASSESSMENT A.Psycho-social health of patients (uncertainty, anxiety, informational needs, mood, coping, quality of life, social activity, ..):

B.Physical health of patients (eg. functional status, self-care, self-efficacy, independence, ..): Selfcare deficits / self-developed / no / telephone interview / 6 weeks after discharge. Blood glucose level / Hba1c-level / unclear / blood sample / 3 months after discharge.

C.Other consumer oriented outcomes (eg. treatment adherence, knowledge, adverse events, ..): Knowledge / Diabetes knowledge scale (Dunn,1984) / yes / telephone interview / 6 weeks after discharge.

D. Health service delivery oriented outcomes (eg. hospital readmission, health services utilization, ..): 
Tu 1993 (Continued)

\author{
·CONTACT WITH AUTHOR \\ ·POWER CALCULATION? \\ -RECORD IF THE STUDY WAS TRANSLATED FROM A LANGUAGE OTHER THAN ENGLISH. \\ -RECORD IF THE STUDY WAS A DUPLICATE PUBLICATION.
}

\title{
Risk of bias
}

\begin{tabular}{lll}
\hline Bias & Authors' judgement & Support for judgement \\
\hline Allocation concealment? & Unclear risk & B - Unclear \\
\hline
\end{tabular}

Weaver 2001

Methods

\section{DETAILS OF STUDY}

AIM OF INTERVENTION: to reinforce education, answer questions, and support the patient and family in regard to the post-operative recovery process.

AIM OF STUDY: to evaluate the telephone follow-up program; it was sought to compare 2 groups of cardiac surgery patients: those who received usual care and those who received usual care and telephone follow-up calls for 1 month after discharge, with regard to satisfaction with continuity of care, depression, recidivism, and complications.

\section{STUDY DESIGN: CCT.}

METHODS OF RECRUITMENT OF PARTICIPANTS: all eligible cardiac surgery patients were invited to participate. The resulting convenience sample was then randomly divided to control and intervention group.

INCLUSION CRITERIA FOR PARTICIPATION IN STUDY: 21 years and older/ discharged home 3 to 7 days after surgery, able to read, speak and understand English.

EXCLUSION CRITERIA FOR PARTICIPATION IN STUDY: not specified.

INFORMED CONSENT OBTAINED? yes.

ETHICAL APPROVAL? unclear.

FUNDING: yes.

ASSESSMENT OF STUDY QUALITY

ALLOCATION CONCEALMENT: unclear.

EPOC- QUALITY CRITERIA 2002: C.high risk of bias.

METHOD OF GENERATING RANDOMISATION SCHEDULE: not specified.

METHOD OF CONCEALMENT OF ALLOCATION: not specified.

BLINDING:

-PARTICIPANTS: no.

-PROVIDER/S: no.

.OUTCOME ASSESSOR/S: no.

INTENTION TO TREAT ANALYSIS: not stated.

BASELINE COMPARABILITY OF INTERVENTION AND CONTROL GROUPS: not stated. 
Weaver 2001 (Continued)

STATISTICAL METHODS AND THEIR APPROPRIATENESS: not stated.

CONSUMER INVOLVEMENT: not stated.

Participants

DESCRIPTION: cardiac patients / surgery patients.

GEOGRAPHIC LOCATION: USA.

SETTING: discharged home from an acute care setting.

NUMBER OF PARTICIPANTS:

ELIGIBLE: 90.

RANDOMISED TO INTERVENTION: 44.

RANDOMISED TO CONTROL: 46.

INCLUDED IN ANALYSIS INTERVENTION GROUP: 44.

INCLUDED IN ANALYSIS CONTROL GROUP: 46.

AGE: RANGE OR MEAN (SD): 63.

GENDER (\% MALE): 70.

ETHNICITY: unclear.

PRINCIPAL HEALTH PROBLEM OR DIAGNOSIS: coronary artery disease.

OTHER HEALTH PROBLEM/S: not specified.

TREATMENT RECEIVED/RECEIVING: CABG or valve replacement.

OTHER SOCIAL/DEMOGRAPHIC DETAILS: 20\% living alone, 55\% retired.

Interventions

DETAILS OF INTERVENTION: within 2 days of discharge, a telephone call was made by a cardiovascular stepdown nurse. This nurse called once weekly for one month or more frequently if the needs or concerns of the patient or his family so required. The nurse was allowed to talk with either the patient or a family member. A standardized assessment sheet guided the calls. Areas of focus included respiratory, cardiac, and neurologic systems, fluid status, pain management, sleep, nutrition, elimination, activity, self-care, psychosocial status, wound management and patient knowledge.

DETAILS OF CONTROL: usual care.

CO-INTERVENTION? no.

DETAILS OF CO-INTERVENTIONS:

DELIVERY OF INTERVENTION

Frequency: 4-?

First time at day 2 after discharge.

Period: 1 month.

PROVIDERS: nurse.

INTERVENTION QUALITY: unclear.

FIDELITY/INTEGRITY: good.

Outcomes

NUMBER OF OUTCOMES: 4

OUTCOME / TOOL / TOOL VALIDATED / METHOD OF ASSESSMENT / TIME OF ASSESSMENT

A.Psycho-social health of patients (uncertainty, anxiety, informational needs, mood, coping, quality of life, social activity, ...): Satisfaction / self-developed / unclear / postal questionnaire / 1 month after discharge. Depression / geriatric depression scale / unclear / postal questionnaire / 1 month after discharge.

B.Physical health of patients (eg. functional status, self-care, self-efficacy, independence, ...):

Telephone follow-up, initiated by a hospital-based health professional, for postdischarge problems in patients discharged from hospital

Copyright $\odot 2010$ The Cochrane Collaboration. Published by John Wiley \& Sons, Ltd. 
D. Health service delivery oriented outcomes (eg. hospital readmission, health services utilization, ...): Readmission / self-developed / no / hospital record / 1 month after discharge.

ED-visits / self-developed / no / hospital record / 1 month after discharge.

\begin{tabular}{|c|c|c|}
\hline \multirow[t]{5}{*}{ Notes } & \multicolumn{2}{|c|}{ CHANGES IN TRIAL PROTOCOL } \\
\hline & \multicolumn{2}{|c|}{.CONTACT WITH AUTHOR } \\
\hline & \multicolumn{2}{|l|}{ POWER CALCULATION? } \\
\hline & \multicolumn{2}{|c|}{ ·RECORD IF THE STUDY WAS TRANSLATED FROM A LANGUAGE OTHER THAN ENGLISH. } \\
\hline & \multicolumn{2}{|c|}{ ·RECORD IF THE STUDY WAS A DUPLICATE PUBLICATION. } \\
\hline \multicolumn{3}{|l|}{ Risk of bias } \\
\hline Bias & Authors' judgement & Support for judgement \\
\hline Allocation concealment? & Unclear risk & B - Unclear \\
\hline
\end{tabular}

Cells containing blanks under a heading mean there was no information on that item in the trial report.

No studies reported methods of follow-up for non-respondents, or adverse events.

$\mathrm{RCT}$ : randomised controlled trial

CCT: controlled clinical trial (quasi-randomised)

Characteristics of excluded studies [ordered by study ID]

\begin{tabular}{|c|c|}
\hline Study & Reason for exclusion \\
\hline Aadalen 1998 & Intervention is not a telephone follow-up by a hospital based professional to patient. \\
\hline Aaronson 1996 & Study does not concern patients discharged from hospital to home. \\
\hline Alcaide 1990 & Study does not concern patients discharged from hospital to home. \\
\hline Alfaro 1997 & Does not present results of a controlled trial. \\
\hline Allen 2002 & Outcomes are not measured at least once within 3 months after discharge. \\
\hline anonymous 1995a & Does not present results of a controlled trial. \\
\hline anonymous 1995b & Does not present results of a controlled trial. \\
\hline anonymous $1995 \mathrm{c}$ & Does not present results of a controlled trial. \\
\hline anonymous $1996 a$ & Does not present results of a controlled trial. \\
\hline anonymous 1996b & Does not present results of a controlled trial. \\
\hline anonymous 1997 & Does not present results of a controlled trial. \\
\hline anonymous 1998 & Does not present results of a controlled trial. \\
\hline anonymous 2001a & Does not present results of a controlled trial. \\
\hline
\end{tabular}




\begin{tabular}{|c|c|}
\hline Study & Reason for exclusion \\
\hline anonymous 2001b & Does not present results of a controlled trial. \\
\hline anonymous 2001c & Does not present results of a controlled trial. \\
\hline Appel 2002 & Study does not concern patients discharged from hospital to home. \\
\hline Arthur 2002 & Outcomes are not measured at least once within 3 months after discharge. \\
\hline Austin 1996 & Study does not concern patients discharged from hospital to home. \\
\hline Avlund 2002 & Intervention is not a telephone follow-up by a hospital based professional to patient. \\
\hline Bailey 1998 & Does not present results of a controlled trial. \\
\hline Barsevick 2002 & Study does not concern patients discharged from hospital to home. \\
\hline Bartlett 1976 & Does not present results of a controlled trial. \\
\hline Bean 1995 & Does not present results of a controlled trial. \\
\hline Beard 1978 & Does not present results of a controlled trial. \\
\hline Bedeian 1996 & Does not present results of a controlled trial. \\
\hline Beebe 2001 & Study does not concern patients discharged from hospital to home. \\
\hline Behrns 2000 & Intervention is not a telephone follow-up by a hospital based professional to patient. \\
\hline Benatar 2003 & Intervention is not a telephone follow-up by a hospital based professional to patient. \\
\hline Bennett 2000 & Does not present results of a controlled trial. \\
\hline Bergstrom 2000 & Does not present results of a controlled trial. \\
\hline Berkman 1999 & Effects of the TFU can not be isolated and analyzed to some degree. \\
\hline Berry 2002 & Does not present results of a controlled trial. \\
\hline Biermann 2000 & Study does not concern patients discharged from hospital to home. \\
\hline Biermann 2002 & Study does not concern patients discharged from hospital to home. \\
\hline Blake 1990 & Study does not concern patients discharged from hospital to home. \\
\hline Blue 2001 & Intervention is not a telephone follow-up by a hospital based professional to patient. \\
\hline Booker 2000 & Does not present results of a controlled trial. \\
\hline Bostelman 1994 & Does not present results of a controlled trial. \\
\hline Boter 1998 & Does not present results of a controlled trial. \\
\hline Bourbeau 2003 & The intervention does not take place at least once within the first month after hospital disharge. \\
\hline Branch 1999 & Study does not concern patients discharged from hospital to home. \\
\hline
\end{tabular}




\begin{tabular}{|c|c|}
\hline Study & Reason for exclusion \\
\hline Brandis 1998 & Does not present results of a controlled trial. \\
\hline Brandt 1994 & Does not present results of a controlled trial. \\
\hline Brooks 2002 & Study does not concern patients discharged from hospital to home. \\
\hline Caison 1997 & Study does not concern patients discharged from hospital to home. \\
\hline Cave 1989 & Does not present results of a controlled trial. \\
\hline Celestino 1998 & Does not present results of a controlled trial. \\
\hline Chong 2003 & Does not present results of a controlled trial. \\
\hline Chow 2001 & Does not present results of a controlled trial. \\
\hline Cleuren 2000 & Does not present results of a controlled trial. \\
\hline Cooper 2000 & Does not present results of a controlled trial. \\
\hline Craddock 1999 & Effects of the TFU can not be isolated and analyzed to some degree. \\
\hline Dale 1997 & Does not present results of a controlled trial. \\
\hline Dantas 2002 & Does not present results of a controlled trial. \\
\hline Dardik 1997 & Study does not concern patients discharged from hospital to home. \\
\hline DeBusk 1985 & Effects of the TFU can not be isolated and analyzed to some degree. \\
\hline DeBusk 1994 & Effects of the TFU can not be isolated and analyzed to some degree. \\
\hline Dellasega 2000 & Intervention is not a telephone follow-up by a hospital based professional to patient. \\
\hline Delores 2000 & Effects of the TFU can not be isolated and analyzed to some degree. \\
\hline Doolittle 1997 & Does not present results of a controlled trial. \\
\hline Dunn 1995 & Intervention is not a telephone follow-up by a hospital based professional to patient. \\
\hline Eaton 2002 & Study does not concern patients discharged from hospital to home. \\
\hline Edwards 1997 & Intervention is not a telephone follow-up by a hospital based professional to patient. \\
\hline Elliott 1998 & Does not present results of a controlled trial. \\
\hline Engelman 1994 & Effects of the TFU can not be isolated and analyzed to some degree. \\
\hline Estey 1990 & Study does not concern patients discharged from hospital to home. \\
\hline Evans 1985 & Effects of the TFU can not be isolated and analyzed to some degree. \\
\hline Ezenkwele 2003 & Intervention is not a telephone follow-up by a hospital based professional to patient. \\
\hline Faithfull 2001 & Effects of the TFU can not be isolated and analyzed to some degree. \\
\hline
\end{tabular}




\begin{tabular}{|c|c|}
\hline Study & Reason for exclusion \\
\hline Farrero 2001 & Study does not concern patients discharged from hospital to home. \\
\hline Ferrigno 2001 & Intervention is not a telephone follow-up by a hospital based professional to patient. \\
\hline Fitzgerald 1985 & Study does not concern patients discharged from hospital to home. \\
\hline Fleming 2002 & Study does not concern patients discharged from hospital to home. \\
\hline Fowler 1992 & Does not present results of a controlled trial. \\
\hline Frank 1986 & Outcomes are not measured at least once within 3 months after discharge. \\
\hline Frank 1987 & Outcomes are not measured at least once within 3 months after discharge. \\
\hline Frasure-Smith 1985 & Study does not concern patients discharged from hospital to home. \\
\hline Frasure-Smith 1991 & Intervention is not a telephone follow-up by a hospital based professional to patient. \\
\hline Frasure-Smith 1992 & Intervention is not a telephone follow-up by a hospital based professional to patient. \\
\hline Frasure-Smith 1997 & Intervention is not a telephone follow-up by a hospital based professional to patient. \\
\hline Frasure-Smith 2002 & Intervention is not a telephone follow-up by a hospital based professional to patient. \\
\hline Fretwell 1990 & Effects of the TFU can not be isolated and analyzed to some degree. \\
\hline Friedman 1998a & Study does not concern patients discharged from hospital to home. \\
\hline Friedman 1998b & Does not present results of a controlled trial. \\
\hline Fukuda 1999 & Study does not concern patients discharged from hospital to home. \\
\hline Gagnon 1997 & Effects of the TFU can not be isolated and analyzed to some degree. \\
\hline Gagnon 1999 & Intervention is not a telephone follow-up by a hospital based professional to patient. \\
\hline Gagnon 2002 & Effects of the TFU can not be isolated and analyzed to some degree. \\
\hline Gallagher 2003 & Effects of the TFU can not be isolated and analyzed to some degree. \\
\hline Galt 2000 & Does not present results of a controlled trial. \\
\hline Gamboa 2002a & Study does not concern patients discharged from hospital to home. \\
\hline Gamboa 2002b & Study does not concern patients discharged from hospital to home. \\
\hline Garland 1992 & Does not present results of a controlled trial. \\
\hline Garnett 1981 & Study does not concern patients discharged from hospital to home. \\
\hline Genev 1990 & Study does not concern patients discharged from hospital to home. \\
\hline Gilliss 1993 & Effects of the TFU can not be isolated and analyzed to some degree. \\
\hline Glasgow 1995 & Study does not concern patients discharged from hospital to home. \\
\hline
\end{tabular}




\begin{tabular}{|c|c|}
\hline Study & Reason for exclusion \\
\hline Glasgow 1996 & Study does not concern patients discharged from hospital to home. \\
\hline Glasgow 1997 & Study does not concern patients discharged from hospital to home. \\
\hline Glasgow 2000 & Study does not concern patients discharged from hospital to home. \\
\hline Glasgow 2001 & Does not present results of a controlled trial. \\
\hline Glasgow 2002 & Study does not concern patients discharged from hospital to home. \\
\hline Goes 2002 & Does not present results of a controlled trial. \\
\hline Gortner 1988 & Effects of the TFU can not be isolated and analyzed to some degree. \\
\hline Grancelli 2003 & Does not present results of a controlled trial. \\
\hline Greineder 1995 & Does not present results of a controlled trial. \\
\hline Greineder 1999 & Study does not concern patients discharged from hospital to home. \\
\hline Griffin 1989 & Study does not concern patients discharged from hospital to home. \\
\hline Grunfeld 1999 & Study does not concern patients discharged from hospital to home. \\
\hline Gulliford 1997 & Study does not concern patients discharged from hospital to home. \\
\hline Harrison 1999 & Study does not concern patients discharged from hospital to home. \\
\hline Hartmann 1996 & Does not present results of a controlled trial. \\
\hline Hasseler 2002 & Does not present results of a controlled trial. \\
\hline Hauber 2002 & Intervention is not a telephone follow-up by a hospital based professional to patient. \\
\hline Hayes 2001a & Study does not concern patients discharged from hospital to home. \\
\hline Hayes 2001b & Does not present results of a controlled trial. \\
\hline Heidenreich 1999 & Study does not concern patients discharged from hospital to home. \\
\hline Heller 1993 & Outcomes are not measured at least once within 3 months after discharge. \\
\hline Hendricks 2000 & Study does not concern patients discharged from hospital to home. \\
\hline Hernandez 2003 & Intervention is not a telephone follow-up by a hospital based professional to patient. \\
\hline Hickey 1996 & Intervention is not a telephone follow-up by a hospital based professional to patient. \\
\hline Higgins 2001 & Effects of the TFU can not be isolated and analyzed to some degree. \\
\hline Hillebrand 1996 & Outcomes are not measured at least once within 3 months after discharge. \\
\hline Hornblow 1980 & Does not present results of a controlled trial. \\
\hline Hoskins 1985 & Does not present results of a controlled trial. \\
\hline
\end{tabular}




\begin{tabular}{|c|c|}
\hline Study & Reason for exclusion \\
\hline Hoskins 2001 & Intervention is not a telephone follow-up by a hospital based professional to patient. \\
\hline Houzard 1998 & Intervention is not a telephone follow-up by a hospital based professional to patient. \\
\hline Hui 2000 & Study does not concern patients discharged from hospital to home. \\
\hline Intagliata 1976 & Does not present results of a controlled trial. \\
\hline Jahanshahi 1994 & Study does not concern patients discharged from hospital to home. \\
\hline James 1994 & Does not present results of a controlled trial. \\
\hline Joffe 1995 & Study does not concern patients discharged from hospital to home. \\
\hline Johnson 2000a & Study does not concern patients discharged from hospital to home. \\
\hline Johnson 2000b & Outcomes are not measured at least once within 3 months after discharge. \\
\hline Johnson $2000 \mathrm{c}$ & Does not present results of a controlled trial. \\
\hline Jolly 2003 & Does not present results of a controlled trial. \\
\hline Jones 1988b & Does not present results of a controlled trial. \\
\hline Jones 1997 & Does not present results of a controlled trial. \\
\hline Jowers 2000 & Does not present results of a controlled trial. \\
\hline Kasper 2002 & Outcomes are not measured at least once within 3 months after discharge. \\
\hline Kelly 1999 & Does not present results of a controlled trial. \\
\hline King 1991 & Study does not concern patients discharged from hospital to home. \\
\hline Kirkman 1994 & Study does not concern patients discharged from hospital to home. \\
\hline Kirscht 1981 & Study does not concern patients discharged from hospital to home. \\
\hline Kokubu 1999 & Study does not concern patients discharged from hospital to home. \\
\hline Kokubu 2000 & Study does not concern patients discharged from hospital to home. \\
\hline Korner-Bitensky 1994 & Intervention is not a telephone follow-up by a hospital based professional to patient. \\
\hline Kramer 2003 & Effects of the TFU can not be isolated and analyzed to some degree. \\
\hline Kunik 2001 & Study does not concern patients discharged from hospital to home. \\
\hline Lando 2001 & Study does not concern patients discharged from hospital to home. \\
\hline Laramee 2003 & Effects of the TFU can not be isolated and analyzed to some degree. \\
\hline Lear 2001 & Study does not concern patients discharged from hospital to home. \\
\hline Lear 2002 & Study does not concern patients discharged from hospital to home. \\
\hline
\end{tabular}




\begin{tabular}{|c|c|}
\hline Study & Reason for exclusion \\
\hline Lee 1999 & Study does not concern patients discharged from hospital to home. \\
\hline Liew 1994 & Intervention is not a telephone follow-up by a hospital based professional to patient. \\
\hline Litzelman 1993 & Study does not concern patients discharged from hospital to home. \\
\hline Lundblad 2001 & Does not present results of a controlled trial. \\
\hline Lynch 2003 & Study does not concern patients discharged from hospital to home. \\
\hline MacMahon 1999 & Does not present results of a controlled trial. \\
\hline Madge 1997 & Intervention is not a telephone follow-up by a hospital based professional to patient. \\
\hline Madonna 1999 & Does not present results of a controlled trial. \\
\hline Maiman 1979 & The intervention does not take place at least once within the first month after hospital disharge \\
\hline Maisiak 1996a & Study does not concern patients discharged from hospital to home. \\
\hline Maisiak 1996b & Study does not concern patients discharged from hospital to home. \\
\hline Manian 1993 & Does not present results of a controlled trial. \\
\hline Marcus 1998 & Does not present results of a controlled trial. \\
\hline Marrero 1995 & Study does not concern patients discharged from hospital to home. \\
\hline Mason 1998 & Does not present results of a controlled trial. \\
\hline Maunsell 1996 & Effects of the TFU can not be isolated and analyzed to some degree. \\
\hline McCorkle 2000 & Effects of the TFU can not be isolated and analyzed to some degree. \\
\hline McDonald 2002 & Effects of the TFU can not be isolated and analyzed to some degree. \\
\hline McGrath 2002 & Does not present results of a controlled trial. \\
\hline McIntosh 1994 & Does not present results of a controlled trial. \\
\hline McMurray 1998 & Does not present results of a controlled trial. \\
\hline McNamara 1995 & Does not present results of a controlled trial. \\
\hline Meenan 1998 & Effects of the TFU can not be isolated and analyzed to some degree. \\
\hline Miller 1995 & Outcomes are not measured at least once within 3 months after discharge. \\
\hline Miller 1997a & Outcomes are not measured at least once within 3 months after discharge. \\
\hline Miller 1997b & Does not present results of a controlled trial. \\
\hline Miller 2002a & Study does not concern patients discharged from hospital to home. \\
\hline Miller 2002b & Does not present results of a controlled trial. \\
\hline
\end{tabular}




\begin{tabular}{|c|c|}
\hline Study & Reason for exclusion \\
\hline Miranda 2002 & Does not present results of a controlled trial. \\
\hline Mishel 2002 & Outcomes are not measured at least once within 3 months after discharge. \\
\hline Mohlman 2003 & Study does not concern patients discharged from hospital to home. \\
\hline Mohr 2000 & Study does not concern patients discharged from hospital to home. \\
\hline Moran 1998 & Effects of the TFU can not be isolated and analyzed to some degree. \\
\hline Morrison 2001 & Intervention is not a telephone follow-up by a hospital based professional to patient. \\
\hline Napolitano 2002 & Study does not concern patients discharged from hospital to home. \\
\hline Naylor 1999 & Effects of the TFU can not be isolated and analyzed to some degree. \\
\hline Nelson 2001 & Does not present results of a controlled trial. \\
\hline Newman 2002 & Does not present results of a controlled trial. \\
\hline Nicklin 1986 & Does not present results of a controlled trial. \\
\hline Nijdam 1999 & Does not present results of a controlled trial. \\
\hline Northouse 2002 & Study does not concern patients discharged from hospital to home. \\
\hline O'Neill 2001 & Does not present results of a controlled trial. \\
\hline Oddone 1999 & Outcomes are not measured at least once within 3 months after discharge. \\
\hline Oh 2003 & Study does not concern patients discharged from hospital to home. \\
\hline Pal 1998 & Does not present results of a controlled trial. \\
\hline Pal 2001 & Does not present results of a controlled trial. \\
\hline Palmer 2001 & Study does not concern patients discharged from hospital to home. \\
\hline Palmer 2002 & Study does not concern patients discharged from hospital to home. \\
\hline Peterson 2002 & Does not present results of a controlled trial. \\
\hline Pidd 2000 & Does not present results of a controlled trial. \\
\hline Poncia 2000 & Does not present results of a controlled trial. \\
\hline Powell 2001 & Study does not concern patients discharged from hospital to home. \\
\hline Powers 1983 & Study does not concern patients discharged from hospital to home. \\
\hline Proctor 2000 & Does not present results of a controlled trial. \\
\hline Pugh 1999 & Outcomes are not measured at least once within 3 months after discharge. \\
\hline Racelis 1998 & The intervention does not take place at least once within the first month after hospital disharge. \\
\hline
\end{tabular}




\begin{tabular}{|c|c|}
\hline Study & Reason for exclusion \\
\hline Rakowski 1994 & Does not present results of a controlled trial. \\
\hline Rauh 1999 & Effects of the TFU can not be isolated and analyzed to some degree. \\
\hline Rawl 1998 & Outcomes are not measured at least once within 3 months after discharge. \\
\hline Rawl 2002 & Effects of the TFU can not be isolated and analyzed to some degree. \\
\hline Rene 1992 & Study does not concern patients discharged from hospital to home. \\
\hline Rich 1995 & Effects of the TFU can not be isolated and analyzed to some degree. \\
\hline Riegel 1996 & Does not present results of a controlled trial. \\
\hline Riegel 2000 & Effects of the TFU can not be isolated and analyzed to some degree. \\
\hline Rieger 1995 & Intervention is not a telephone follow-up by a hospital based professional to patient. \\
\hline Ries 2003 & Study does not concern patients discharged from hospital to home. \\
\hline Rigotti 1997 & Effects of the TFU can not be isolated and analyzed to some degree. \\
\hline Riley 1989 & Does not present results of a controlled trial. \\
\hline Roberts 1995 & Study does not concern patients discharged from hospital to home. \\
\hline Roglieri 1997 & Effects of the TFU can not be isolated and analyzed to some degree. \\
\hline Romano 2001 & Does not present results of a controlled trial. \\
\hline Rosbe 2000 & Does not present results of a controlled trial. \\
\hline Rosswurm 1998 & Intervention is not a telephone follow-up by a hospital based professional to patient. \\
\hline Ruchlin 2001 & Intervention is not a telephone follow-up by a hospital based professional to patient. \\
\hline Sanders 1997 & Study does not concern patients discharged from hospital to home. \\
\hline Sandgren 2000 & Study does not concern patients discharged from hospital to home. \\
\hline Sandgren 2003 & Study does not concern patients discharged from hospital to home. \\
\hline Sardell 2000 & Does not present results of a controlled trial. \\
\hline Schatz 2003 & Study does not concern patients discharged from hospital to home. \\
\hline Schectman 1994 & Study does not concern patients discharged from hospital to home. \\
\hline Schultz 1993 & Study does not concern patients discharged from hospital to home. \\
\hline Sciamanna 2000 & Does not present results of a controlled trial. \\
\hline Shah 1998 & Does not present results of a controlled trial. \\
\hline Shapiro 1995 & Intervention is not a telephone follow-up by a hospital based professional to patient \\
\hline
\end{tabular}




\begin{tabular}{|c|c|}
\hline Study & Reason for exclusion \\
\hline Shon 2002 & Study does not concern patients discharged from hospital to home. \\
\hline Shu 1996 & Does not present results of a controlled trial. \\
\hline Siegel 1992 & Study does not concern patients discharged from hospital to home. \\
\hline Simon 1997 & Outcomes are not measured at least once within 3 months after discharge. \\
\hline Simon 2003 & Outcomes are not measured at least once within 3 months after discharge. \\
\hline Sluijk 1998 & Does not present results of a controlled trial. \\
\hline Smeenk 1998a & Does not present results of a controlled trial. \\
\hline Smeenk 1998b & Intervention is not a telephone follow-up by a hospital based professional to patient. \\
\hline Smith 1988 & Effects of the TFU can not be isolated and analyzed to some degree. \\
\hline Smith 2002 & Does not present results of a controlled trial. \\
\hline Sneed 1997 & Outcomes are not measured at least once within 3 months after discharge. \\
\hline Soskolne 1993 & Intervention is not a telephone follow-up by a hospital based professional to patient. \\
\hline Sparacino 1997 & Does not present results of a controlled trial. \\
\hline Stanislaw 1994 & Effects of the TFU can not be isolated and analyzed to some degree. \\
\hline Steel 2003 & Intervention is not a telephone follow-up by a hospital based professional to patient. \\
\hline Stevens 1993 & Effects of the TFU can not be isolated and analyzed to some degree. \\
\hline Stevens 2000 & Outcomes are not measured at least once within 3 months after discharge. \\
\hline Stewart 1998 & Intervention is not a telephone follow-up by a hospital based professional to patient. \\
\hline Strecher 1983 & Does not present results of a controlled trial. \\
\hline Strinko 2000 & Does not present results of a controlled trial. \\
\hline Svahn 2002 & Effects of the TFU can not be isolated and analyzed to some degree. \\
\hline Taylor 1990 & Outcomes are not measured at least once within 3 months after discharge. \\
\hline Taylor 1996 & Effects of the TFU can not be isolated and analyzed to some degree. \\
\hline Taylor 1997 & Outcomes are not measured at least once within 3 months after discharge. \\
\hline Taylor-Davis 2000 & Study does not concern patients discharged from hospital to home. \\
\hline Thewissen 2000 & Does not present results of a controlled trial. \\
\hline Thompson 1999 & Study does not concern patients discharged from hospital to home. \\
\hline Tiippana 2003 & Does not present results of a controlled trial. \\
\hline
\end{tabular}




\begin{tabular}{|c|c|}
\hline Study & Reason for exclusion \\
\hline Tkachuk 2003 & Study does not concern patients discharged from hospital to home. \\
\hline Townsend 1996 & Intervention is not a telephone follow-up by a hospital based professional to patient. \\
\hline Turner 1996 & Does not present results of a controlled trial. \\
\hline Tyc 2003 & Study does not concern patients discharged from hospital to home. \\
\hline Valanis 2001 & Does not present results of a controlled trial. \\
\hline Valanis 2002 & Does not present results of a controlled trial. \\
\hline Valanis 2003 & Does not present results of a controlled trial. \\
\hline Vale 2002 & Outcomes are not measured at least once within 3 months after discharge. \\
\hline van Beelen 1996 & Does not present results of a controlled trial. \\
\hline van Elderen 1994 & Effects of the TFU can not be isolated and analyzed to some degree. \\
\hline van Elderen 2001 & Effects of the TFU can not be isolated and analyzed to some degree. \\
\hline Varma 1999 & Intervention is not a telephone follow-up by a hospital based professional to patient. \\
\hline Vogel 1996 & Does not present results of a controlled trial. \\
\hline Vrehen 2000 & Does not present results of a controlled trial. \\
\hline Wade 1998 & Outcomes are not measured at least once within 3 months after discharge. \\
\hline Walker 1999 & Intervention is not a telephone follow-up by a hospital based professional to patient. \\
\hline Warden 2000 & Does not present results of a controlled trial. \\
\hline Wasson 1992 & Study does not concern patients discharged from hospital to home. \\
\hline Weinberger 1991 & Study does not concern patients discharged from hospital to home. \\
\hline Weinberger 1993 & Study does not concern patients discharged from hospital to home. \\
\hline Weinberger 1998 & Does not present results of a controlled trial. \\
\hline Weinstein 1996 & Study does not concern patients discharged from hospital to home. \\
\hline Welch 2000 & Study does not concern patients discharged from hospital to home. \\
\hline Wells 2003 & Study does not concern patients discharged from hospital to home. \\
\hline Wewers 1994 & Study does not concern patients discharged from hospital to home. \\
\hline Wilbourne 1997 & Does not present results of a controlled trial. \\
\hline Wong 2001a & Does not present results of a controlled trial. \\
\hline Wong 2001b & Does not present results of a controlled trial. \\
\hline
\end{tabular}




\begin{tabular}{ll}
\hline Study & Reason for exclusion \\
\hline Wulsin 2002 & Intervention is not a telephone follow-up by a hospital based professional to patient. \\
\hline York 1997 & Effects of the TFU can not be isolated and analyzed to some degree. \\
\hline Young 2000 & Does not present results of a controlled trial. \\
\hline Zahlmann 2002 & Study does not concern patients discharged from hospital to home. \\
\hline Zeegers 1997 & Does not present results of a controlled trial. \\
\hline Zorc 2003 & Intervention is not a telephone follow-up by a hospital based professional to patient. \\
\hline
\end{tabular}

\section{DATA AND ANALYSES}

Comparison 1. Effect of TFU on anxiety in cardiac surgery patients at appr. 1 month after discharge compared to usual care

\begin{tabular}{lllll}
\hline Outcome or subgroup title & $\begin{array}{l}\text { No. of } \\
\text { studies }\end{array}$ & $\begin{array}{l}\text { No. of } \\
\text { partici- } \\
\text { pants }\end{array}$ & Statistical method & Effect size \\
\hline $\begin{array}{l}1 \text { Effect of TFU on anxiety in cardiac surgery patients at } \\
\text { appr. } 1 \text { month after discharge compared to usual care }\end{array}$ & 3 & 278 & $\begin{array}{l}\text { Std. Mean Difference (IV, } \\
\text { Random, 95\% Cl) }\end{array}$ & $-0.47[-1.28$, \\
\hline
\end{tabular}

\section{Analysis 1.1. Comparison 1 Effect of TFU on anxiety in cardiac surgery patients at appr. 1 month after discharge compared to usual care, Outcome 1 Effect of TFU on anxiety in cardiac surgery patients at appr. 1 month after discharge compared to usual care.}

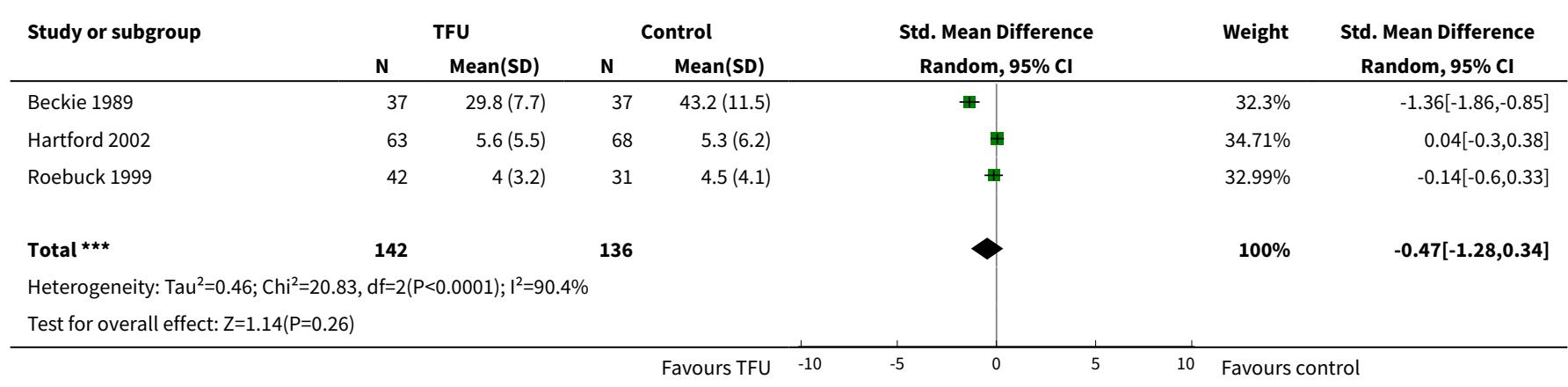


Comparison 2. Effect of TFU on compliance

\begin{tabular}{lllll}
\hline Outcome or subgroup title & $\begin{array}{l}\text { No. of } \\
\text { studies }\end{array}$ & $\begin{array}{l}\text { No. of } \\
\text { partici- } \\
\text { pants }\end{array}$ & Statistical method & Effect size \\
\hline $\begin{array}{l}1 \text { Effect of TFU on compliance in cardiac surgery patients } \\
\text { compared to usual care }\end{array}$ & 2 & 102 & $\begin{array}{l}\text { Risk Ratio (M-H, Random, } \\
95 \% \mathrm{Cl})\end{array}$ & $\begin{array}{l}1.68[0.59, \\
4.78]\end{array}$ \\
\hline $\begin{array}{l}2 \text { Effect of TFU on compliance (making an appointment) in } \\
\text { ED patients compared to usual care }\end{array}$ & 3 & 1039 & $\begin{array}{l}\text { Risk Ratio (M-H, Random, } \\
95 \% \mathrm{Cl})\end{array}$ & $\begin{array}{l}1.70[0.92, \\
3.14]\end{array}$ \\
\hline $\begin{array}{l}3 \text { Effect of TFU on compliance (keeping an appointment) in } \\
\text { ED patients compared to usual care }\end{array}$ & 3 & 820 & $\begin{array}{l}\text { Risk Ratio (M-H, Random, } \\
95 \% \mathrm{Cl})\end{array}$ & $\begin{array}{l}1.58[1.01, \\
2.48]\end{array}$ \\
\hline
\end{tabular}

Analysis 2.1. Comparison 2 Effect of TFU on compliance, Outcome 1 Effect of TFU on compliance in cardiac surgery patients compared to usual care.

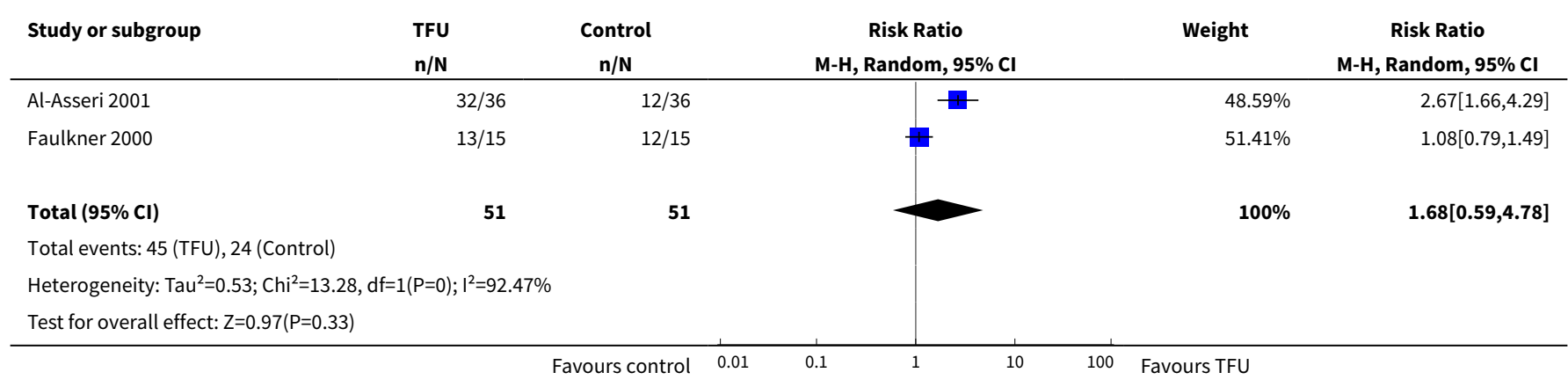

Analysis 2.2. Comparison 2 Effect of TFU on compliance, Outcome 2 Effect of TFU on compliance (making an appointment) in ED patients compared to usual care.

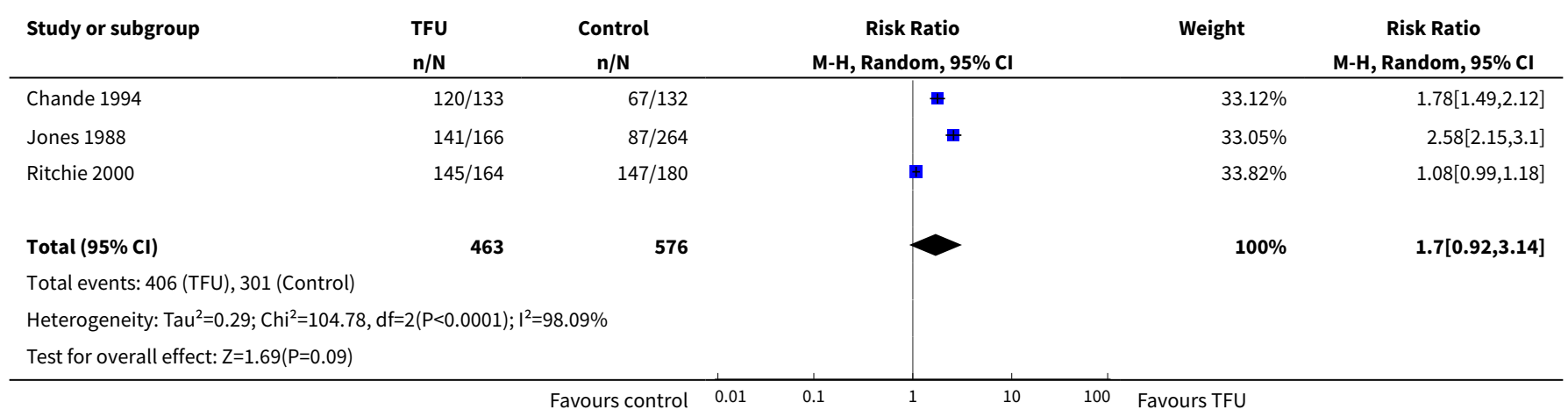


Analysis 2.3. Comparison 2 Effect of TFU on compliance, Outcome 3 Effect of TFU on compliance (keeping an appointment) in ED patients compared to usual care.

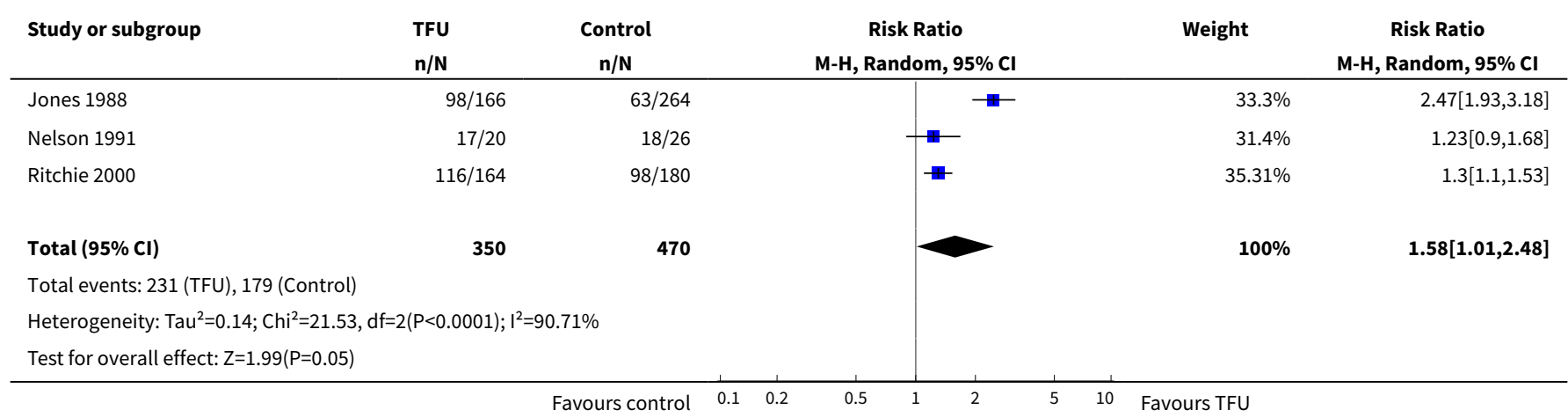

Comparison 3. Effect of TFU on knowledge in cardiac patients compared to control condition

\begin{tabular}{lllll}
\hline Outcome or subgroup title & $\begin{array}{l}\text { No. of } \\
\text { studies }\end{array}$ & $\begin{array}{l}\text { No. of } \\
\text { partici- } \\
\text { pants }\end{array}$ & Statistical method & Effect size \\
\hline $\begin{array}{l}1 \text { Effect of TFU on knowledge in cardiac patients at around } \\
6 \text { weeks post discharge compared to control condition }\end{array}$ & 3 & 185 & $\begin{array}{l}\text { Std. Mean Difference (IV, } \\
\text { Random, 95\% Cl) }\end{array}$ & $\begin{array}{l}1.44[-0.25, \\
3.13]\end{array}$ \\
\hline
\end{tabular}

Analysis 3.1. Comparison 3 Effect of TFU on knowledge in cardiac patients compared to control condition, Outcome 1 Effect of TFU on knowledge in cardiac patients at around 6 weeks post discharge compared to control condition.

\begin{tabular}{|c|c|c|c|c|c|c|c|}
\hline \multirow[t]{2}{*}{ Study or subgroup } & \multicolumn{2}{|c|}{ TFU } & \multicolumn{2}{|c|}{ Control } & \multirow{2}{*}{$\begin{array}{c}\text { Std. Mean Difference } \\
\text { Random, } 95 \% \mathrm{Cl}\end{array}$} & \multirow[t]{2}{*}{ Weight } & \multirow{2}{*}{$\begin{array}{c}\text { Std. Mean Difference } \\
\text { Random, } 95 \% \mathrm{CI}\end{array}$} \\
\hline & $\mathbf{N}$ & Mean(SD) & $\mathbf{N}$ & Mean(SD) & & & \\
\hline Barnason 1995 & 30 & $88(8.5)$ & 30 & $85.7(8.1)$ & \# & $33.75 \%$ & $0.28[-0.23,0.79]$ \\
\hline Beckie 1989 & 37 & $67.7(1.4)$ & 37 & $52.4(6.3)$ & 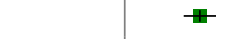 & $32.77 \%$ & $3.33[2.61,4.04]$ \\
\hline Garding 1988 & 25 & $12.5(7.8)$ & 26 & $5.9(9.2)$ & \# & $33.49 \%$ & $0.76[0.19,1.33]$ \\
\hline 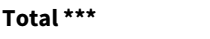 & 92 & & 93 & & & $100 \%$ & $1.44[-0.25,3.13]$ \\
\hline \multicolumn{8}{|c|}{ Heterogeneity: $\mathrm{Tau}^{2}=2.12 ; \mathrm{Chi}^{2}=48.67, \mathrm{df}=2(\mathrm{P}<0.0001) ; \mathrm{I}^{2}=95.89 \%$} \\
\hline \multicolumn{8}{|c|}{ Test for overall effect: $Z=1.67(P=0.09)$} \\
\hline
\end{tabular}

\section{Comparison 4. Effect of TFU on readmissions}

\begin{tabular}{lllll}
\hline Outcome or subgroup title & $\begin{array}{l}\text { No. of } \\
\text { studies }\end{array}$ & $\begin{array}{l}\text { No. of } \\
\text { partici- } \\
\text { pants }\end{array}$ & Statistical method & Effect size \\
\hline $\begin{array}{l}1 \text { Effect of TFU on readmissions in cardiac patients } \\
\text { compared to usual care }\end{array}$ & 3 & 616 & $\begin{array}{l}\text { Risk Ratio (M-H, Random, } \\
95 \% \mathrm{Cl})\end{array}$ & $\begin{array}{l}0.75[0.41, \\
1.36]\end{array}$ \\
\hline $\begin{array}{l}2 \text { Effect of TFU on readmissions in surgery patients } \\
\text { compared to control condition }\end{array}$ & 4 & 460 & $\begin{array}{l}\text { Risk Ratio (M-H, Random, } \\
95 \% \mathrm{Cl})\end{array}$ & $0.65[0.28$, \\
$1.55]$
\end{tabular}

Telephone follow-up, initiated by a hospital-based health professional, for postdischarge problems in patients discharged from hospital 
Analysis 4.1. Comparison 4 Effect of TFU on readmissions, Outcome 1 Effect of TFU on readmissions in cardiac patients compared to usual care.

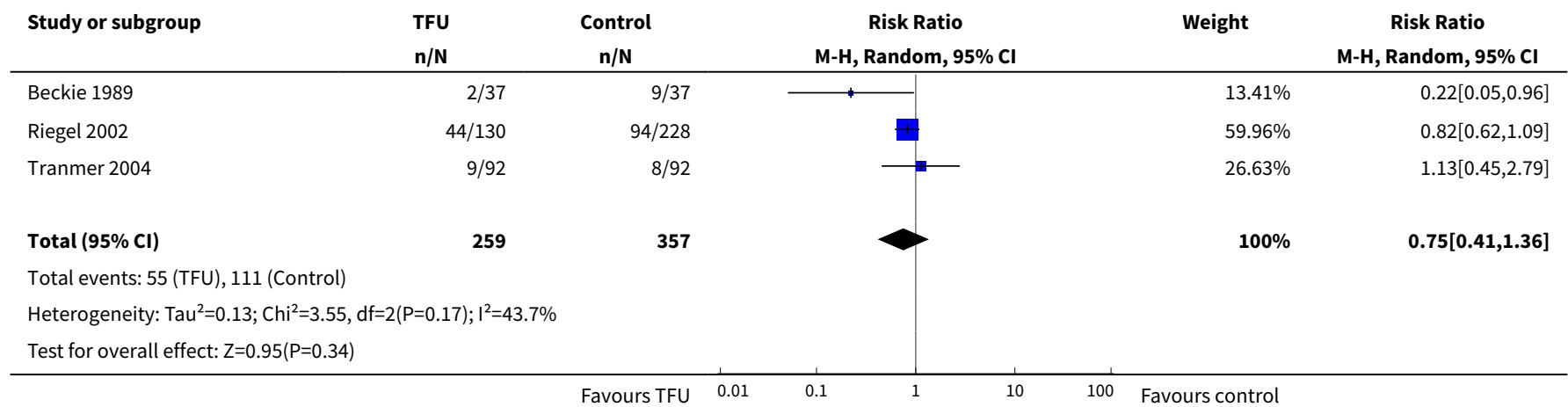

Analysis 4.2. Comparison 4 Effect of TFU on readmissions, Outcome 2 Effect of TFU on readmissions in surgery patients compared to control condition.

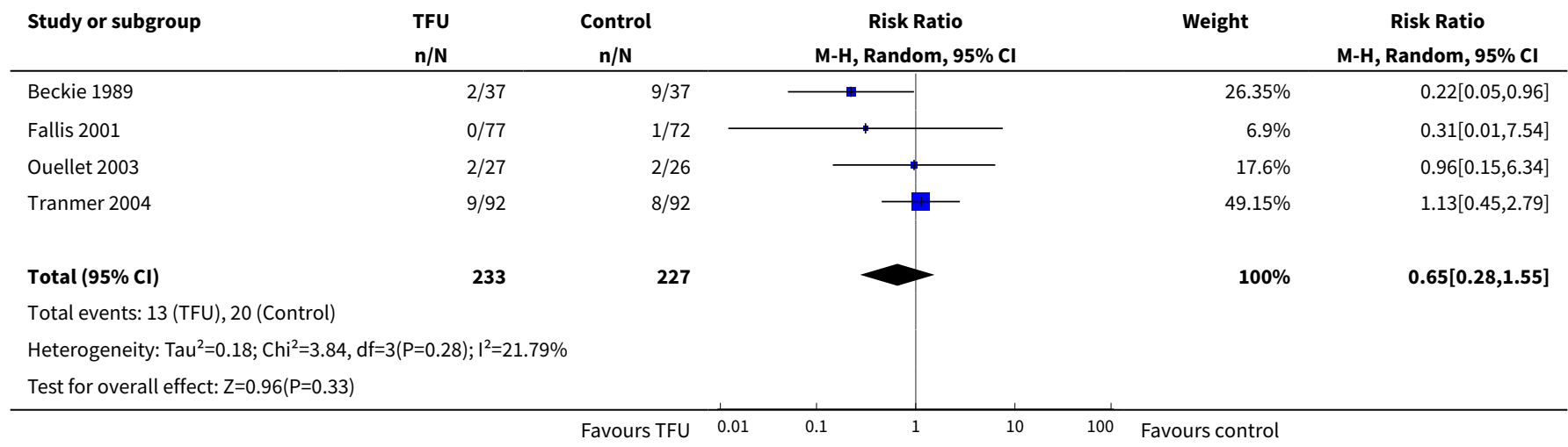

Comparison 5. Effect of TFU on ED visits in surgery patients compared to control condition

\begin{tabular}{llll}
\hline Outcome or subgroup title & $\begin{array}{l}\text { No. of } \\
\text { studies }\end{array}$ & $\begin{array}{l}\text { No. of } \\
\text { partici- } \\
\text { pants }\end{array}$ & Statistical method \\
\hline $\begin{array}{l}1 \text { Effect of TFU on ED visits in surgery patients compared } \\
\text { to control condition }\end{array}$ & 2 & 333 & $\begin{array}{l}\text { Risk Ratio (M-H, Random, } \\
95 \% \mathrm{Cl})\end{array}$ \\
\hline
\end{tabular}

Telephone follow-up, initiated by a hospital-based health professional, for postdischarge problems in patients discharged from hospital 
Analysis 5.1. Comparison 5 Effect of TFU on ED visits in surgery patients compared to control condition, Outcome 1 Effect of TFU on ED visits in surgery patients compared to control condition.

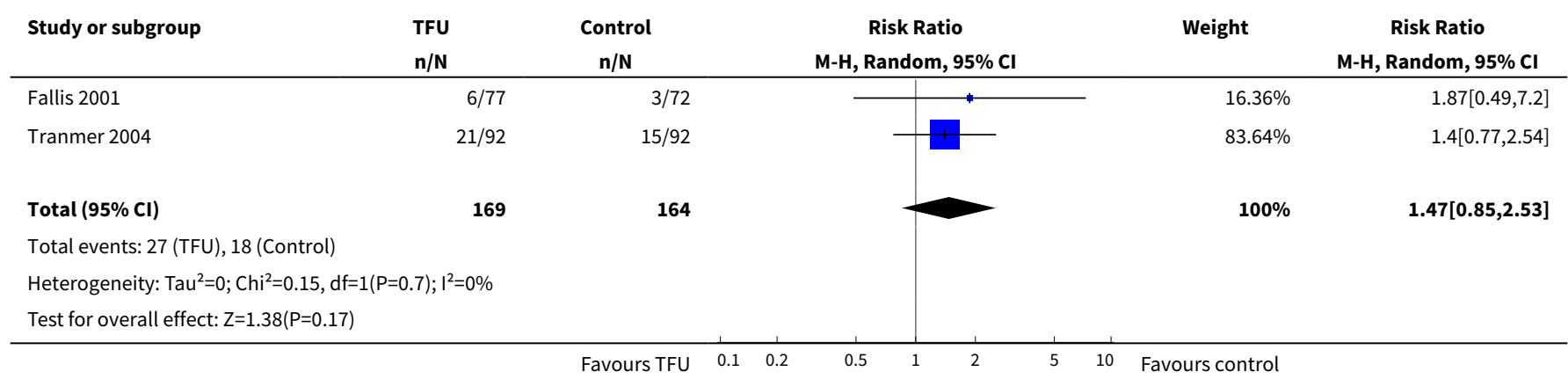

\section{ADDITIONAL TABLES}

Table 1. Outcome/patient combinations for which pooling was considered

\begin{tabular}{|c|c|c|c|c|c|}
\hline Outcome category & $\begin{array}{l}\text { Cardiac } \\
\text { patients }\end{array}$ & $\begin{array}{l}\text { Surgery } \\
\text { patients }\end{array}$ & $\begin{array}{l}\text { ED pa- } \\
\text { tients }\end{array}$ & $\begin{array}{l}\text { Paedi- } \\
\text { atric pa- } \\
\text { tients }\end{array}$ & $\begin{array}{l}\text { Neurol- } \\
\text { ogy pa- } \\
\text { tients }\end{array}$ \\
\hline \multicolumn{6}{|c|}{ PSYCHO-SOCIAL HEALTH OUTCOMES } \\
\hline -anxiety & 3 & 3 & & & \\
\hline -satisfaction & 5 & 6 & & & \\
\hline -depression & 2 & 2 & & & \\
\hline \multicolumn{6}{|c|}{ OTHER CONSUMER ORIENTED OUTCOMES } \\
\hline -compliance & 2 & 2 & 4 & 3 & \\
\hline -knowledge & 3 & 2 & & & \\
\hline \multicolumn{6}{|c|}{ HEALTH SERVICES ORIENTED OUTCOMES } \\
\hline -readmissions & 4 & 5 & & & 2 \\
\hline -ED-visits & 2 & 3 & & & \\
\hline
\end{tabular}

\section{APPENDICES}

\section{Appendix 1. PubMed controlled trials search strategy}

(randomised controlled trial [pt] OR controlled clinical trial [pt] OR randomised controlled trials [mh] OR random allocation [mh] OR double-blind method [mh] OR single-blind method [mh] OR clinical trial [pt] OR clinical trials [mh] OR ("clinical trial" [tw]) OR ((singl* [tw] OR doubl* [tw] OR trebl* [tw] OR tripl* [tw]) AND (mask* [tw] OR blind* [tw])) OR ("latin square" [tw]) OR placebos [mh] OR placebo* [tw] OR random* ${ }^{\star} \mathrm{tw}$ ] OR research design [mh:noexp]) OR comparative study [mh] OR evaluation studies [mh] OR follow-up studies [mh] OR prospective studies [mh] OR cross-over studies [mh] OR control* [tw] OR prospectiv* [tw] OR volunteer* [tw]) NOT (animal [mh] NOT human $[\mathrm{mh}])$ 


\section{Appendix 2. PubMed topic-specific strategy}

"telecommunications"[MeSH Terms] OR tele?communication* [tw] OR electronic communication* OR "telephone"[MeSH Terms] OR telephon^ $[\mathrm{tw}$ ] OR phone[tw] OR phone call* OR follow-up call* OR call?back [tw] OR calls [tw] OR calling [tw] OR call [tw] OR tele?health OR tele?medicine

AND

"patient discharge"[MeSH Terms] OR ((patient* OR client* OR consumer ${ }^{\star}$ OR recipient ${ }^{\star}$ OR subject $\left.{ }^{\star}\right)$ AND discharg AR $^{\star}$ ORospital discharg ${ }^{\star}$ OR "hospital discharge"[tw] OR "aftercare"[MeSH Terms] OR aftercare [tw] OR "continuity of patient care"[MeSH Terms] OR convales*[tw] OR recover ${ }^{\star}[\mathrm{tw}]$ OR post?operative care OR ((patient ${ }^{\star}$ OR client ${ }^{\star}$ OR consumer ${ }^{\star}$ OR recipient ${ }^{\star}$ OR subject ${ }^{\star}$ OR care?giver $^{\star}$ OR carer ${ }^{\star}$ OR famil ${ }^{\star}$ ) AND (inform* OR educat* OR instruct* OR counsel* OR advise* OR advice OR reassur ${ }^{\star}$ OR support*)) OR information* need ${ }^{\star}$ [tw] OR post?hospital*

\section{Appendix 3. BiomedCentral search strategy}

(("telecommunications" OR tele?communication* [tw] OR electronic communication* OR "telephone" OR telephon* [tw] OR phone[tw] OR phone call* OR follow-up call* OR call?back [tw] OR calls [tw] OR calling [tw] OR call [tw] OR tele?health OR tele?medicine)) AND ("patient discharge" OR ((patient ${ }^{\star}$ OR client* OR consumer* OR recipient* OR subject*) AND discharg*) OR hospital discharg* OR "hospital discharge"[tw] OR "aftercare" OR aftercare [tw] OR "continuity of patient care" OR convales* [tw] OR recover*[tw] OR post?operative care OR ((patient ${ }^{\star}$ OR client ${ }^{\star}$ OR consumer ${ }^{\star}$ OR recipient ${ }^{\star}$ OR subject ${ }^{\star}$ OR care? giver ${ }^{\star}$ OR carer ${ }^{\star}$ OR famil ${ }^{\star}$ ) AND (inform ${ }^{\star}$ OR educat ${ }^{\star}$ OR instruct $^{\star}$ OR counsel ${ }^{\star}$ OR advise ${ }^{\star}$ OR advice OR reassur ${ }^{\star}$ OR support $\left.{ }^{\star}\right)$ ) OR information ${ }^{\star}$ need $^{\star}$ [tw] OR post?hospital ${ }^{\star}$ )

\section{Appendix 4. CENTRAL search strategy}

1. $(((()((()(($ TELECOMMUNICATIONS Or TELECOMMUNICATION*) OR (ELECTRONIC and COMMUNICATION*)) OR TELEPHONE) OR TELEPHON $^{\star}$ ) OR PHONETW) OR (PHONE AND CALL*)) OR (FOLLOW-UP AND CALL*)) OR CALLBACK) OR CALLS) OR CALLING) OR CALL) OR TELEHEALTH) OR TELEMEDICINE)

2.COMMUNICATION*:ME

3.TELEPHONE*:ME

4.HOTLINES*:ME

5.TELEMEDICINE*:ME

6.TELECOMMUNICATIONS*:ME

7.PHONE-CALL

8.PHONE-CALLS

9.CALL-BACK

10. $(((((((\# 1$ or \#2) or \#3) or \#4) or \#5) or \#6) or \#7) or \#8) or \#9)

11.PATIENT-DISCHARGE*:ME

12.AFTERCARE*:ME

13.CONTINUITY-OF-PATIENT-CARE*:ME

14.DISCHARG*

15.FOLLOW-UP

16. ((( $\# 11$ or \#12) or \#13) or \#14) or \#15)

17.(\#10 and \#16)

\section{Appendix 5. CINAHL search strategy}

1.DISCHARGE in TI,AB,DE,TP,SH

2.explode "Transfer-Discharge"/ all topical subheadings / all age subheadings

3.DISCHARG* in TI,AB,DE,TP,SH

4.explode "Discharge-Planning"/ all topical subheadings / all age subheadings

5.explode "Early-Patient-Discharge"/ all topical subheadings / all age subheadings

6.explode "After-Care"/ all topical subheadings / all age subheadings

7.AFTERCARE in TI,AB,DE,TP,SH

8.AFTER?CARE in TI,AB,DE,TP,SH

9.POST?HOSPITAL* in TI,AB,DE,TP,SH

10.explode "Continuity-of-Patient-Care"/ all topical subheadings / all age subheadings

11.CONVALES ${ }^{*}$ in $\mathrm{TI}, \mathrm{AB}, \mathrm{DE}, \mathrm{TP}, \mathrm{SH}$

12.explode "Recovery"/ all topical subheadings / all age subheadings

13.REASSUR* in TI,AB,DE,TP,SH

14.INFORMATION* NEED* in TI,AB,DE,TP,SH

15.explode "Information-Needs"/ all topical subheadings / all age subheadings

16.POST?DISCHARGE in TI,AB,DE,TP,SH

17.explode "Patient-Discharge"/ all topical subheadings / all age subheadings

$18 . \# 1$ or \#2 or \#3 or \#4 or \#5 or \#6 or \#7 or \#8 or \#9 or \#10 or \#11 or \#12 or \#13 or \#14 or \#15 or \#16 or \#17 19.RANDOMIZED CONTROLLED TRIAL in TI,AB,DE,TP,SH

Telephone follow-up, initiated by a hospital-based health professional, for postdischarge problems in patients discharged from hospital 
20.RANDOMIZED CLINICAL TRIAL in TI,AB,DE,TP,SH

21.CLINICAL TRIAL in TI,AB,DE,TP,SH

22.explode "Clinical-Trials"/ all topical subheadings / all age subheadings

23.CONTROLLED CLINICAL TRIAL in TI,AB,DE,TP,SH

24.CONTROLLED TRIAL in TI,AB,DE,TP,SH

25.RANDOM* in TI,AB,DE,TP,SH

26.CROSS-OVER STUDIES in TI,AB,DE,TP,SH

27.CROSS-OVER STUDY in TI,AB,DE,TP,SH

28.RESEARCH DESIGN in TI,AB,DE,TP,SH

29.explode "Research"/ all topical subheadings / all age subheadings

30.explode "Study-Design"/ all topical subheadings / all age subheadings

31 . \#19 or \#20 or \#21 or \#22 or \#23 or \#24 or \#25 or \#26 or \#27 or \#28 or \#29 or \#30

32.TELEPHONE in TI,AB,DE,TP,SH

33.explode "Telephone-Information-Services"/ all topical subheadings / all age subheadings

34.TELE?COMMUNICATION* in TI,AB,DE,TP,SH

35.explode "Telecommunications"/ all topical subheadings / all age subheadings

36.explode "Telemedicine"/ all topical subheadings / all age subheadings

37.ELECTRONIC COMMUNICATION in TI,AB,DE,TP,SH

38.telephon*

39.PHONE in TI,AB,DE,TP,SH

40.explode "Telephone"/ all topical subheadings / all age subheadings

41.PHONE CALL* in TI,AB,DE,TP,SH

42.FOLLOW?UP CALL* in TI,AB,DE,TP,SH

43.CALL?BACK in TI,AB,DE,TP,SH

44.CALLING in TI,AB,DE,TP,SH

45.CALLS in TI,AB,DE,TP,SH

46.CALL in TI,AB,DE,TP,SH

47.TELE?HEALTH in TI,AB,DE,TP,SH

48.explode "Telehealth"/ all topical subheadings / all age subheadings

49.explode "Telenursing"/ all topical subheadings / all age subheadings

50 . \#32 or \#33 or \#34 or \#35 or \#36 or \#37 or \#38 or \#39 or \#40 or \#41 or \#42 or \#43 or \#44 or \#45 or \#46 or \#47 or \#48 or \#49

51.\#18 and \#31 and \#50

\section{Appendix 6. EMBASE search strategy}

1 exp Hospital Discharge/

2 exp AFTERCARE/

3 aftercare.mp. or exp AFTERCARE/

4 continuity care.mp.

5 discharge planning.mp. [mp=title, abstract, subject headings, drug trade name, original title, device manufacturer, drug manufacturer name]

6 hospital discharge.mp. [mp=title, abstract, subject headings, drug trade name, original title, device manufacturer, drug manufacturer name]

7 (discharg\$ or patient\$ discharge or post\#hospital or aftercare or continuity of care or continuity of patient care or convales\$ or recover or reassur\$ or information\$ need).mp. [mp=title, abstract, subject headings, drug trade name, original title, device manufacturer, drug manufacturer name]

81 or 2 or 3 or 4 or 5 or 6 or 7

9 exp TELEPHONE/ or telephone.mp.

10 telecommunication.mp. or exp TELECOMMUNICATION/

11 (tele\#communication $\$$ or electronic communication or telephon\$ or phone or phone call\$ or follow\#up call\$ or call\#back or calls or calling or call or tele\#health or tele\#medicine or tele\#nursing).mp. [mp=title, abstract, subject headings, drug trade name, original title, device manufacturer, drug manufacturer name]

129 or 10 or 11

138 and 12

14 Randomized Controlled Trial/

15 Clinical Trial/

16 (randomized controlled trial or controlled clinical trial or randomized controlled trials or random allocation or double\#blind or single\#blind or clinical trial\$ or random\$ or research design or follow-up stud\$ or prospective stud\$ or cross-over stud\$ or comparative stud\$).mp. [mp=title, abstract, subject headings, drug trade name, original title, device manufacturer, drug manufacturer name]

1714 or 15 or 16

1813 and 17

Telephone follow-up, initiated by a hospital-based health professional, for postdischarge problems in patients discharged from hospital 


\section{Appendix 7. ERIC search strategy}

(explode "Research-" in DEM,DER) and ((discharg* or patient ${ }^{\star}$ discharge or post?hospital or aftercare or continuity of care or continuity of patient care or convales* or recover or reassur ${ }^{\star}$ or information* need $\left.{ }^{\star}\right)$ and (((explode "Telephone-Communications-Systems" in DEM,DER) or (explode "Telephone-Instruction" in DEM,DER)) or (telecommunication* or tele?communication* or electronic communication or telephon* or phone or phone call* or follow? up call* or call?back or calls or calling or call or tele?health or tele?medicine or tele?nursing) or (explode "Telecommunications-" in DEM,DER)))

\section{Appendix 8. Invert search strategy}

Telefoon OR telefonisch OR telefonische OR telefoontje OR telefoneren OR telefoneer OR telefoneert OR telefoneerde OR telefoneerden OR opbellen OR nabellen

\section{Appendix 9. LILACS search strategy}

( telefon ) or "TELEFONE" or "TELEFONEMA" or "TELEFONIA" or "TELEFONICA" or "TELEFONICAS" or "TELEFONICO" or "TELEFONICOS" or "TELEFONISTAS" or "TELEFONO" or "TELEFONOS" or "TELEINFORMATICA" or "TELEINFORMATICS" or "TELEMATHIC" or "TELEMATICA" or "TELEMATICAS" or "TELEMATICS" or "TELEMEDECINE" or "TELEMEDICINA" or "TELEMEDICINE" or "TELENURSE" or "TELESALUD" or "TELEPHONE" or "TELEPHONES" or "TELEPHONIC" [Title words] or ( telefon ) or "TELEFONE" or "TELEFONEMA" or "TELEFONIA" or "TELEFONICA" or "TELEFONICAS" or "TELEFONICO" or "TELEFONICOS" or "TELEFONISTAS" or "TELEFONO" or "TELEFONOS" or "TELEINFORMATICA" or "TELEINFORMATICS" or "TELEMATHIC" or "TELEMATICA" or "TELEMATICAS" or "TELEMATICS" or "TELEMEDECINE" or "TELEMEDICINA" or "TELEMEDICINE" or "TELENURSE" or "TELESALUD" or "TELEPHONE" or "TELEPHONES" or "TELEPHONIC" [SUbject descriptor] or ( telefon ) or "TELEFONE" or "TELEFONEMA" or "TELEFONIA" or "TELEFONICA" or "TELEFONICAS" or "TELEFONICO" or "TELEFONICOS" or "TELEFONISTAS" or "TELEFONO" or "TELEFONOS" or "TELEINFORMATICA" or "TELEINFORMATICS" or "TELEMATHIC" or "TELEMATICA" or "TELEMATICAS" or "TELEMATICS" or "TELEMEDECINE" or "TELEMEDICINA" or "TELEMEDICINE" or "TELENURSE" or "TELESALUD" or "TELEPHONE" or "TELEPHONES" or "TELEPHONIC" [Abstract words]

\section{Appendix 10. PICARTA search strategy}

((ziekenhuisontslag OR (ontslag BIJ/4 ziekenhuis) OR (zorg BIJ/4 ontslag) OR postontslag* OR nazorg OR (continuit* BIJ/4 zorg)) OR (discharge\#planning) OR (discharge \# planning) OR (hospital \# discharge) OR (aftercare) OR (patient* BIJ discharge) OR (post\#hospital) OR (post \# hospital) OR (continuity \# care) OR follow\#up OR (follow \# up)) AND (telefo* OR telepho* OR call OR phone OR tele\#health OR tele\#medicine OR tele\#communicat* OR tele\#nursing)

\section{Appendix 11. PsycINFO / PsycLIT search strategy}

(("Discharge-Planning" in DE) or ("Hospital-Discharge" in DE) or ("Aftercare-" in DE) or (discharg* or patient* discharge or post? hospital or aftercare or continuity of care or continuity of patient care or convales* or recover or reassur* or information ${ }^{\star}$ need $\left.{ }^{\star}\right)$ ) and (("Telecommunications-Media" in DE) or (telecommunication* or tele?communication* or electronic communication or telephon* or phone or phone call* or follow?up call* or call?back or calls or calling or call or tele?health or tele?medicine or tele?nursing)) and (((RANDOM) or (RANDOM-) or (RANDOM-ASSIGNMENT)) or (randomized controlled trial* or controlled clinical trial* or random* or double? blind or clinical trial* or research design or comparative study or cross?over stud ${ }^{\star}$ ) or (("Empirical-Methods" in DE) or ("ExperimentalDesign" in DE) or ("Treatment-Effectiveness-Evaluation" in DE)))

\section{Appendix 12. Science Citation Index search strategy}

(discharg* OR patient* discharge OR post?hospital OR aftercare OR continuity of care OR continuity of patient care OR convales ${ }^{\star}$ OR recover OR reassur ${ }^{\star}$ OR information ${ }^{\star}$ need ${ }^{\star}$ ) AND (telecommunication* OR tele?communication* OR electronic communication OR telephon ${ }^{\star}$ OR phone OR phone call ${ }^{\star}$ OR telephone follow? up OR follow? up call ${ }^{\star}$ OR call?back OR calls OR calling OR call OR tele?health OR tele?medicine OR tele?nursing)

\section{Appendix 13. Sociofile search strategy}

((telecommunication* or tele?communication* or electronic communication or telephon* or phone or phone call* or follow?up call* or call?back or calls or calling or call or tele?health or tele?medicine or tele?nursing) or ((explode "Telecommunications-" in DE) or (explode "Telephone-Communications" in DE) or (explode "Telephone-Surveys" in DE))) and (((explode "After-Care" in DE) or (explode "Discharge-" in $\mathrm{DE})$ ) or (discharg* or patient ${ }^{\star}$ discharge or post?hospital or aftercare or continuity of care or continuity of patient care or convales* or recover or reassur* or information* need $\left.{ }^{\star}\right)$ )

\section{WHAT'S NEW}

\begin{tabular}{lll}
\hline Date & Event & Description \\
\hline 4 April 2008 & Amended & Converted to new review format. \\
\hline
\end{tabular}

Telephone follow-up, initiated by a hospital-based health professional, for postdischarge problems in patients discharged from hospital 


\section{HIST O RY}

Protocol first published: Issue 4, 2003

Review first published: Issue 4, 2006

\begin{tabular}{lll}
\hline Date & Event & Description \\
\hline 14 May 2004 & Amended & $\begin{array}{l}\text { We added a sixth inclusion criterion to the protocol, that states } \\
\text { that only studies in which the effect of the TFU can be isolated } \\
\text { and analyzed, will be included. This extra inclusion criterium has } \\
\text { no consequences for the search strategy, and no other studies } \\
\text { will be included or excluded than was originally intended. The } \\
\text { extra inclusion criterium was needed because studies wherein } \\
\text { the effect of the TFU cannot be isolated do not add to the aim of } \\
\text { this review. Moreover, the review is not intended to compare TFU } \\
\text { interventions to multi-component interventions. }\end{array}$ \\
\hline
\end{tabular}

\section{CONTRIBUTIONS OFAUTHORS}

Patriek Mistiaen is the lead author of the review and was involved in all stages of the review. He conceived and designed the protocol for the review. He developed the search strategies, performed all searches, screened search results and organised the retrieval of the papers. He screened retrieved papers against inclusion criteria, appraised the quality of the papers, abstracted data and wrote to authors of papers for additional information. Patriek Mistiaen coordinated the data management, entered data into RevMan, did the data-analysis and wrote the text of the review.

Else Poot is co-author of the review and was involved in all stages of the review, especially by given substantial comments in the design of the protocol, search strategies, data collection form and final text of the review. She screened retrieved papers against inclusion criteria, appraised the quality of the papers, checked all data-entry and data-analysis and made a substantial contribution in the writing of the final text.

\section{DECLARATIONSOF INTEREST}

One of the review authors (PM) is also an co-author of an included study (Boter 2000) in the review.

\section{SOURCES OF SUPPORT}

\section{Internal sources}

- NIVEL, Netherlands Institute for Health Care Services Research, Netherlands.

\section{External sources}

- No sources of support supplied

\section{INDEX TERMS}

\section{Medical Subject Headings (MeSH)}

*Patient Discharge; *Telephone; Aftercare [ ${ }^{*}$ methods] [standards]; Hospitals

\section{MeSH check words}

Humans 